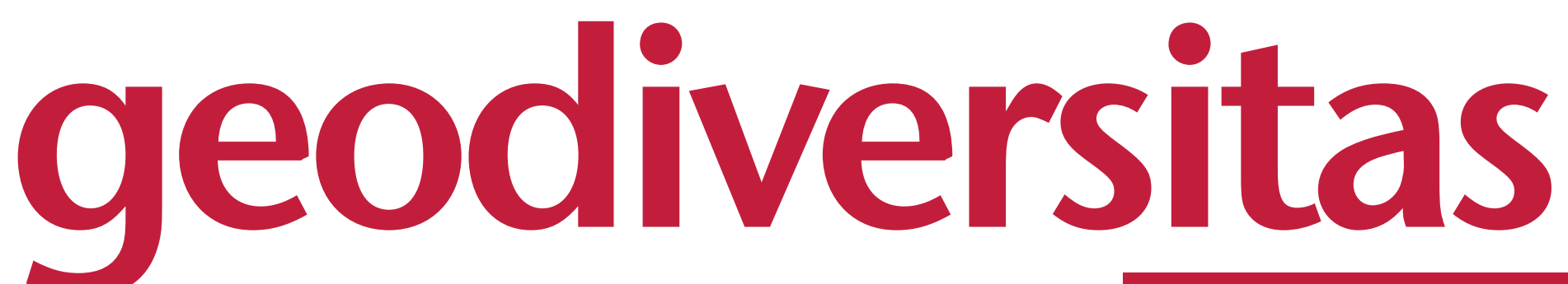

$2019 \cdot 41 \cdot 2$
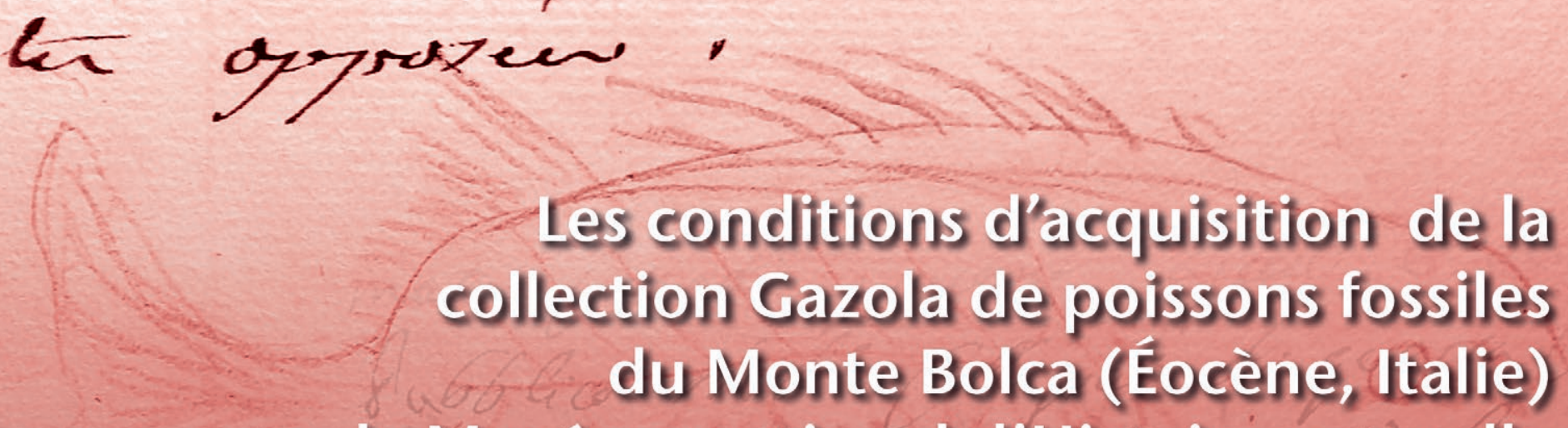
par le Muséum national d histoire naturelle

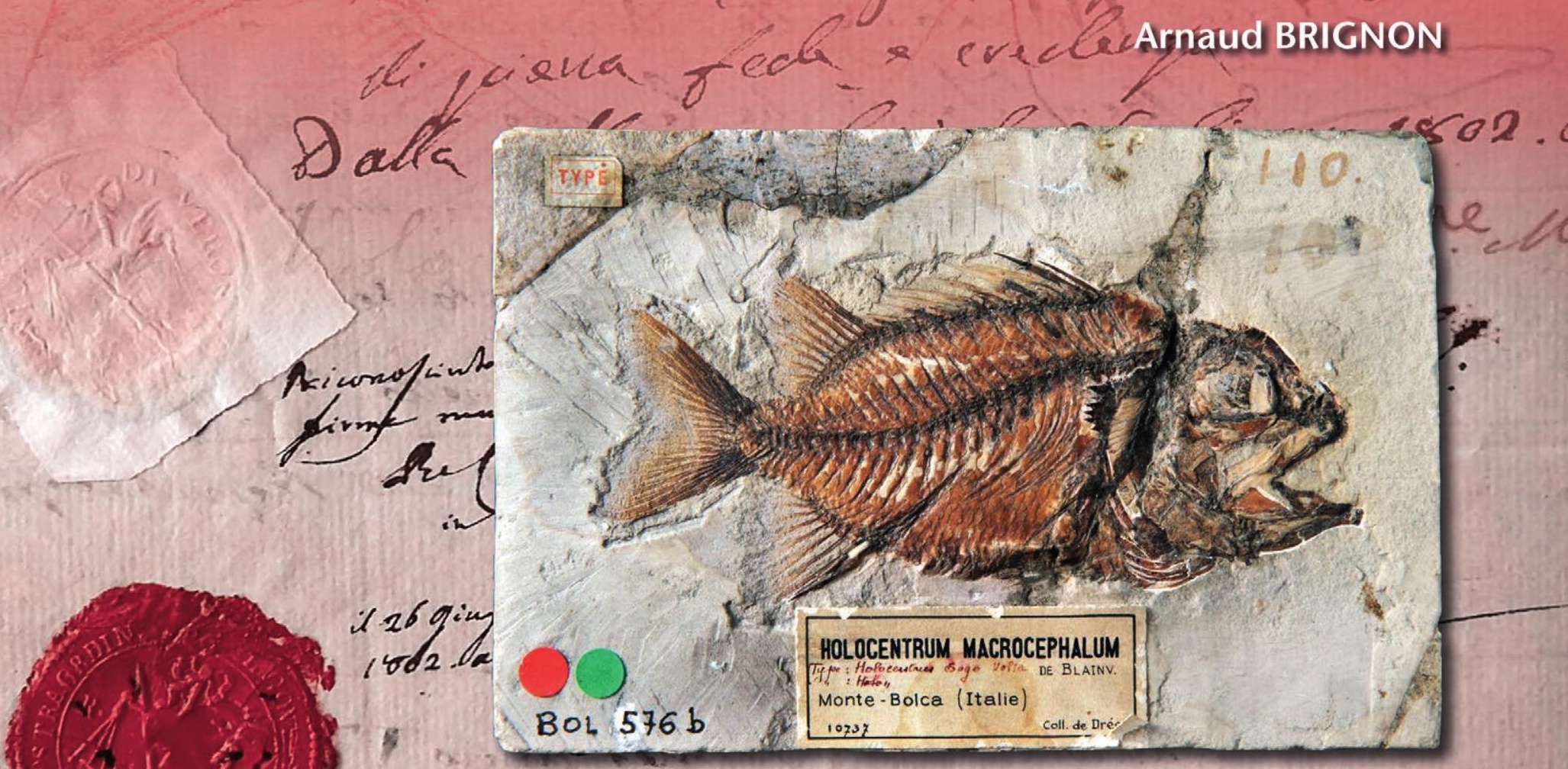

Shilano 1. Suglio 1802. Rumo I:

Si certitica che le vuddette firme vono vere, e i fir.

= mati quali vi quaticicano.

I Conigtiere thimitro seli Interno

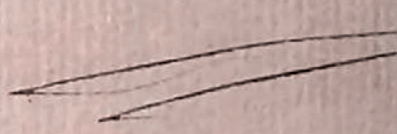


DiRECTEUR DE LA PUBLICATION: Bruno David,

Président du Muséum national d'Histoire naturelle

RÉdACTEUR EN CHEF / EDITOR-IN-CHIEF: Didier Merle

ASSISTANTS DE RÉDACTION / AsSISTANT EDITORS: Emmanuel Côtez (geodiv@mnhn.fr); Anne Mabille

Mise EN PAGE / PAGE LAYOUT: Emmanuel Côtez

COMITÉ SCIENTIFIQUE / SCIENTIFIC BOARD:

Christine Argot (MNHN, Paris)

Beatrix Azanza (Museo Nacional de Ciencias Naturales, Madrid)

Raymond L. Bernor (Howard University, Washington DC)

Alain Blieck (USTL, Villeneuve d'Ascq)

Henning Blom (Uppsala University)

Jean Broutin (UPMC, Paris)

Gaël Clément (MNHN, Paris)

Ted Daeschler (Academy of Natural Sciences, Philadelphie)

Bruno David (MNHN, Paris)

Gregory D. Edgecombe (The Natural History Museum, Londres)

Ursula Göhlich (Natural History Museum Vienna)

Jin Meng (American Museum of Natural History, New York)

Brigitte Meyer-Berthaud (CIRAD, Montpellier)

Zhu Min (Chinese Academy of Sciences, Pékin)

Isabelle Rouget (UPMC, Paris)

Sevket Sen (MNHN, Paris)

Stanislav Štamberg (Museum of Eastern Bohemia, Hradec Králové)

Paul Taylor (The Natural History Museum, Londres)

COUVERTURE / COVER:

Montage d'après les Figures de l'article/made from article Figures.

Geodiversitas est indexé dans / Geodiversitas is indexed in:

- Science Citation Index Expanded (SciSearch ${ }^{\circledR}$ )

- ISI Alerting Services ${ }^{\circledR}$

- Current Contents ${ }^{\circledR}$ / Physical, Chemical, and Earth Sciences ${ }^{\circledR}$

- Scopus ${ }^{\circledR}$

Geodiversitas est distribué en version électronique par / Geodiversitas is distributed electronically by:

- BioOne ${ }^{\circledR}$ (http://www.bioone.org)

Les articles ainsi que les nouveautés nomenclaturales publiés dans Geodiversitas sont référencés par / Articles and nomenclatural novelties published in Geodiversitas are referenced by:

- ZooBank ${ }^{\circledR}$ (http://zoobank.org)

Geodiversitas est une revue en flux continu publiée par les Publications scientifiques du Muséum, Paris Geodiversitas is a fast track journal published by the Museum Science Press, Paris

Les Publications scientifiques du Muséum publient aussi / The Museum Science Press also publish:

Adansonia, Zoosystema, Anthropozoologica, European Journal of Taxonomy, Naturae, Cryptogamie sous-sections Algologie, Bryologie, Mycologie

Diffusion - Publications scientifiques Muséum national d'Histoire naturelle

CP $41-57$ rue Cuvier F-75231 Paris cedex 05 (France)

Tél. : 33 (0)1407948 05 / Fax: 33 (0)14079 3840

diff.pub@mnhn.fr / http://sciencepress.mnhn.fr

(C) Publications scientifiques du Muséum national d'Histoire naturelle, Paris, 2019

ISSN (imprimé / print): 1280-9659/ ISSN (électronique / electronic): 1638-9395 


\title{
Les conditions d'acquisition de la collection Gazola de poissons fossiles du Monte Bolca (Éocène, Italie) par le Muséum national d'Histoire naturelle
}

\author{
Arnaud BRIGNON \\ 5 villa Jeanne d'Arc, F-92340 Bourg-la-Reine (France) \\ arnaud.brignon@yahoo.com
}

MOTS CLÉS

Histoire de la paléontologie, cabinets d'histoire naturelle, Monte Bolca, paléoichtyologie, Chondrichthyes,

Osteichthyes,

Actinopterygii, lectotypifications.
Soumis le 11 juin 2018 | accepté le 26 septembre 2018 | publié le 25 janvier 2019

Brignon A. 2019. - Les conditions d'acquisition de la collection Gazola de poissons fossiles du Monte Bolca (Éocène, Italie) par le Muséum national d'Histoire naturelle. Geodiversitas 41 (2): 11-123. https://doi.org/10.5252/geodiversitas2019v41a2. http://geodiversitas.com/41/2

\section{RÉSUMÉ}

Il est généralement admis que la célèbre collection Gazola de poissons fossiles du Monte Bolca fut confisquée en mai 1797 par les commissaires qui accompagnaient l'Armée d'Italie sous les ordres du général en chef Napoléon Bonaparte. Cette collection (Gazola 1) fut ensuite déposée au Muséum national d'Histoire naturelle (MNHN) en septembre 1798. De nombreux documents inédits conservés aux Archives nationales, émanant principalement du ministère de l'Intérieur, démontrent que Gazola fut indemnisé par une rente viagère dès 1797 à laquelle vint s'ajouter une pension annuelle octroyée par le gouvernement français à partir de 1803. La saisie de la collection Gazola fut donc rétrospectivement légalisée, ce qui explique pourquoi certains commentateurs de l'époque parlèrent d'acquisition ou d'achat. Gazola, qui était propriétaire d'une partie du gisement d'où étaient tirés les fossiles, reconstitua rapidement une seconde collection. Il ne semblait pas éprouver une trop vive rancœur envers le gouvernement français puisqu'en 1803, il offrit à Bonaparte, alors Premier Consul, une partie de cette nouvelle collection pour compléter la première qui avait été acquise par le Muséum en 1798. Le naturaliste Louis Augustin Guillaume Bosc fut mandaté pour récupérer à Vérone, durant l'été 1803, les poissons fossiles offerts par Gazola. Il dressa à cette occasion le catalogue d'une grande partie de la seconde collection Gazola. Le ministre de l'Intérieur, Jean-Antoine Chaptal, ordonna à Bosc de rapporter la totalité du cabinet d'histoire naturelle de Gazola qui voulait pourtant n'en céder qu'une partie. S'opposant à la décision de son ministre, Napoléon Bonaparte intervint en personne pour donner raison à Gazola et faire annuler la mission de Bosc. Deux années s'écoulèrent et c'est finalement Barthélémy Faujas de Saint-Fond qui se chargea de récupérer à Vérone le lot de poissons sélectionnés par Gazola pour le Muséum, fin octobre 1805, alors que la ville était le théatre des affrontements entre les armées française et autrichienne. Cette collection (Gazola 2) arriva à Paris vers le mois de juin 1806. Le catalogue de Bosc présenté ici dans son intégralité permet d'identifier, dans les collections actuelles du MNHN, quelques spécimens de cette seconde collection. 


\begin{abstract}
The conditions of acquisition of the Gazola's collection of fossil fishes from Monte Bolca (Eocene, Italy) by the Muséum national d'Histoire naturelle.

It is generally considered that the famous Gazola's collection of fossil fishes from Monte Bolca was confiscated in May 1797 by the commissioners who accompanied in Italy the French army under the orders of Napoléon Bonaparte. The collection ("Gazola 1") was then deposited in the Muséum National d'Histoire Naturelle, Paris (MNHN), in September 1798. Many unpublished documents, mainly from the French Ministry of the Interior, preserved in the Archives Nationales, show that Gazola was compensated by a life annuity from 1797 to which was added an annual pension granted by the French government from 1803. The seizure of the Gazola collection was thus retrospectively legalized, which explains why some commentators of the time spoke of an acquisition or a purchase. Gazola, who was the owner of a part of the quarry from which were extracted the fossils, quickly reconstituted a second collection. He did not seem to have felt too much resentment toward the French Government since, in 1803, he offered to Bonaparte, then First Consul, a part of this new collection to complete the first collection acquired by the Museum in 1798. The naturalist Louis Augustin Guillaume Bosc was commissioned to collect in Verona, in the summer of 1803, the fossil fish offered by Gazola. On this occasion, he cataloged a large part of the second Gazola collection. The French Minister of the Interior, Jean-Antoine Chaptal, ordered Bosc to bring back the entire collection of Gazola, who only wanted to give a portion of it. Against the decision of his minister, Napoleon Bonaparte intervened in person to give reason to Gazola and to cancel the mission of Bosc. Two years passed and it was finally Barthélémy Faujas de Saint-Fond who took charge of recovering in Verona the batch of fish selected by Gazola for the Museum, at the end of October 1805, while the city was the scene of clashes between the French and Austrian armies. This collection ("Gazola 2") arrived in Paris in June 1806. The Bosc's catalogue presented here in its entirety makes it possible to identify, in the current collections of the MNHN, some of the specimens of the collection Gazola 2.
\end{abstract}

\section{INTRODUCTION}

Le comte Giovanni Battista Gazola (1757-1834) avait formé un véritable musée de "pétrifications» du Monte Bolca dans son palais, situé Piazzetta Chiavica à Vérone (Gaudant 2011a). 516 poissons fossiles ( 189 doubles empreintes et 138 empreintes seules) de cette collection furent rapportés, en septembre 1798, au Muséum national d'Histoire naturelle, par les commissaires chargés de rechercher des objets de science et d'art qui accompagnaient l'Armée d'Italie sous les ordres du général en chef Bonaparte (Frigo \& Sorbini 1997). Aux poissons, s'ajoutaient 78 plantes fossiles (20 doubles empreintes et 38 empreintes seules), la double empreinte d'une plume et celle d'un insecte, soit un total de 598 spécimens. Une seconde collection offerte par Gazola en 1803 vint également s'ajouter à la première (Gaudant 2011b). Une partie des spécimens ainsi réunis au Muséum fut illustrée dans un ouvrage, l'Ittiolitologia Veronese, œuvre monumentale rédigée par Giovanni Serafino Volta (1754-1842) (Volta 1796-1809; Gaudant 2011a). Ces fossiles servirent également de base à la première étude scientifique sur les poissons fossiles par Henry Marie Ducrotay de Blainville (1818a, b) et à l'ouvrage fondateur de la paléoichtyologie, Recherches sur les poissons fossiles, publié par Louis Agassiz (1807-1873) entre 1833 et 1844.

Comment qualifier le transfert de la première collection Gazola au MNHN où elle fut déposée en 1798 ? Acte de pillage, confiscation, acquisition, achat, don ? Les qualificatifs ne manquent pas dans la littérature suivant les sources et suivant les époques, sans compter la confusion qui fut souvent faite entre cette collection et celle qui fut offerte en 1803 . Barthélémy Faujas de Saint-Fond (1741-1819), un des professeurs administrateurs du MNHN, écrivait que Bonaparte avait fait «l'acquisition" de la collection, laissant ainsi supposer qu'une transaction légale avait été conclue (Faujas de SaintFond 1803: 28, note 1; cité par Gaudant 2011b: 642). Joseph Philippe François Deleuze (1753-1835), dans sa description du Muséum, reprenait également que le gouvernement avait acquis la collection de M. le comte Gazola (Deleuze 1823: 345; cité par Frigo \& Sorbini 1997: 18). Ainsi peut-on encore citer d'autres auteurs se référant à une acquisition ou un achat (Agassiz 1833: vol. 1, 5; Pouchet 1841: 597; Cap 1854: 102). Bartolomeo Giuliari (1761-1842), l'imprimeur et le maître d'œuvre de l'Ittiolitologia Veronese, avait raconté à son petit-fils que Gazola avait offert une partie de sa collection à Bonaparte et qu'en échange, des terres lui avaient été cédées pour l'indemniser, d'abord près de Villanfranca, puis dans le Mantouan (Giuliari 1871: 173; cité par Guerra 2017). Dans les procès-verbaux des assemblées des professeurs du Muséum était annoncé, le 24 messidor de l'an 5 (12 juillet 1797), que la collection avait été achetée (Gaudant 2011b: 642). Volta (1796-1809: 2e partie, LVIII) était au contraire beaucoup plus négatif sur cet épisode: "Il Generale in capo dell'armata Francese Napoleone Bonaparte obbligò il Co. Gazola a cedere in suo potere questa degna collezione» [Le général en charge de l'armée française Napoléon Bonaparte obligea Gazola à lui céder cette digne collection].

Sur la base de quelques documents historiques, un consensus semblait émerger plus récemment pour qualifier le transfert 
de la première collection Gazola à Paris de confiscation illégale (Frigo \& Sorbini 1997; Gaudant 2011a, b; Raux 2013; Capasso 2014: 71). Néanmoins, cette conclusion rend difficile à expliquer, voire incompréhensible, le don de la seconde collection de poissons fossiles que fit Giovanni Battista Gazola en 1803 à Napoléon Bonaparte, alors Premier Consul (Lacour 2014: 147). Il eût en effet fallu une dose exceptionnelle de résignation et de magnanimité comme le supposait Jean Gaudant (2011b), pour que Gazola, victime présumée du vol de sa première collection, vinsse offrir à Bonaparte les spécimens qui manquaient au butin français!

La découverte aux Archives nationales d'un important dossier émanant du ministère de l'Intérieur et regroupant des documents manuscrits rédigés entre 1797 et 1815 , permet d'apporter un nouvel éclairage sur les conditions d'acquisition de la collection Gazola par le Muséum national d'Histoire naturelle. L'objectif de cet article est de présenter ces éléments inédits et de comprendre comment les commissaires accompagnant l'Armée d'Italie, Napoléon Bonaparte et plus généralement le gouvernement français se sont comportés vis-à-vis de Gazola. Plusieurs sources indiquaient par ailleurs qu'une seconde collection avait été offerte à Bonaparte en 1803 mais cet épisode restait jusqu'alors mal connu (Faujas de Saint-Fond 1804; Gaudant 2011b). Les documents présentés ici révèlent les circonstances précises, et pour le moins rocambolesques, dans lesquelles cette seconde collection arriva à Paris.

\section{ABRÉVIATIONS}

ADI Archives départementales de l'Isère, Grenoble;

AN Archives nationales, Pierrefitte-sur-Seine;

MCSNV Museo civico di storia naturale di Verona;

MNHN Muséum national d'Histoire naturelle, Paris.

\section{REMARQUES PRÉLIMINAIRES}

Dans le contexte historique de cet article, le terme "poisson", obsolète d'un point de vue cladistique, est utilisé dans son acception ancienne. Ce groupe paraphylétique englobe donc ici les classes des chondrichthyens (Chondrichthyes) et des actinoptérygiens (Actinopterygii), toutes deux représentées dans la faune éocène du Monte Bolca. La transcription des documents manuscrits numérotés de 1 à 67 est donnée dans l'Annexe 1. Le symbole «|» indique un retour à la ligne.

\section{CONTEXTE HISTORIQUE}

L'histoire des découvertes de fossiles au Monte Bolca remonte au XVIe siècle et a été l'objet d'une abondante littérature (Blot 1969; Gaudant 1999, 2005; Guerra 2012, 2014, 2015, 2016, 2017; Clément 2017; Falipou 2017, 2018; Roghi et al. 2017). La proximité du gisement de la Lastrata (connu aujourd'hui sous le nom de la Pesciara) d'où furent extraits la grande majorité des poissons fossiles favorisa l'émergence à Vérone d'un grand nombre de cabinets d'histoire naturelle dans lesquels étaient conservées ces fameuses " pétrifications " (Pomian 1986; Sorbini 1998; Gaudant 2011a). À la fin du XVIIIe siècle, le comte Giovanni Battista Gazola (Fig. 1) pos-

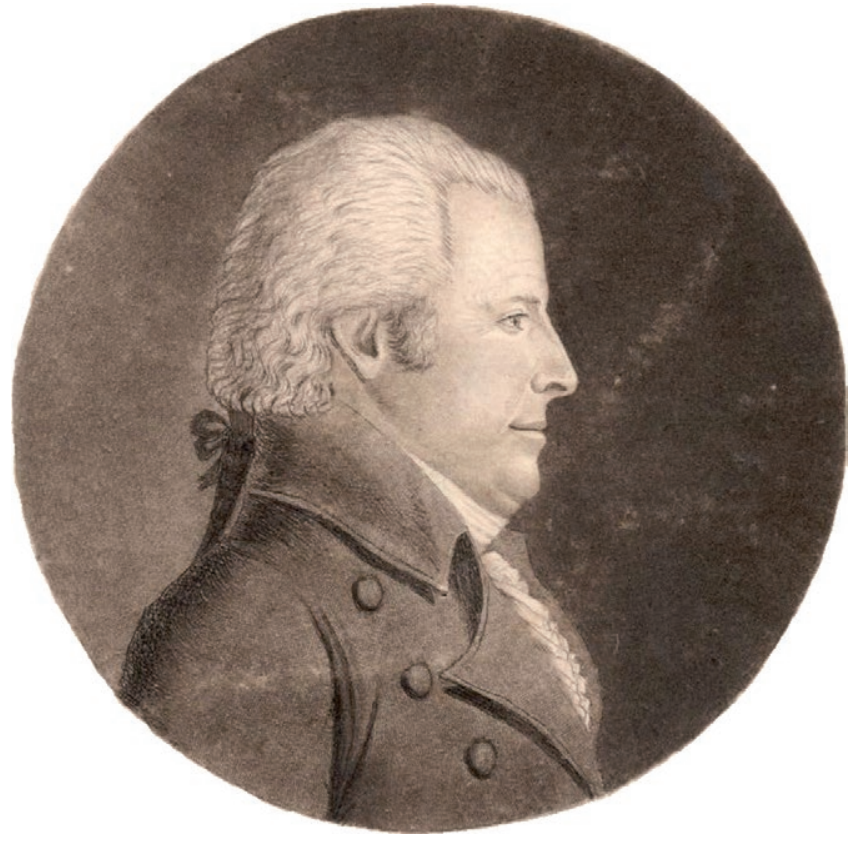

FIG. 1. - Giovanni Battista Gazola (1757-1834). Portrait réalisé à Paris conservé à la Biblioteca Civica di Verona (Stampe 11.c.319).

sédait la plus importante collection de poissons fossiles du Monte Bolca qu'il avait commencé à constituer à partir de 1784 (Frigo \& Sorbini 1997: 9, 10). Tout en organisant des fouilles dans une extension du gisement de la Lastrata dont il était propriétaire depuis 1787 (Falipou 2018), il avait acquis, en 1789, le cabinet du chanoine Gian Giacomo Dionisi (17241808 ) et, en 1791, celui de Vincenzo Bozza, pharmacien à Vérone (Volta 1796-1809: 2e partie, LVIII).

C'est en 1789 que germa l'idée de publier un grand ouvrage sur l'ichtyologie fossile du Véronais qui deviendra l'Ittiolitologia Veronese (Gaudant 2011a). Le projet débuta réellement en 1793 par l'impression d'un prospectus, puis par la gravure de quelques planches dont les prototypes illustrèrent l'ouvrage Lettere recentemente pubblicate sui pesci fossili veronesi publié l'année suivante sous l'impulsion de Gazola (1794). Le début de l'impression de l'Ittiolitologia Veronese en 1796 coïncide avec une période de troubles pour la ville de Vérone. La campagne d'Italie menée par le général Napoléon Bonaparte (Fig. 2) venait de débuter et opposait l'Armée d'Italie de la République française aux forces de l'Empire autrichien et du Royaume de Sardaigne. Le 1er juin 1796, les troupes françaises font leur entrée à Vérone. La ville est brièvement reprise par les Autrichiens du 30 juillet au 8 août pour ensuite repasser sous domination française. Toute la partie occidentale de la Vénétie tomba ainsi aux mains des Français suite aux victoires qui se succédèrent durant les mois de janvier et février 1797. La population, soutenue par les partisans de la République de Venise, se souleva le 17 avril 1797. Au cours de cet épisode, appelé les « Pâques véronaises", des centaines de Français furent massacrés. L'armée française appelée en renfort parvint, après quelques jours, à mâter l'insurrection. De sévères représailles furent infligées à la ville. Le 17 floréal an 5 (6 mai 1797), 


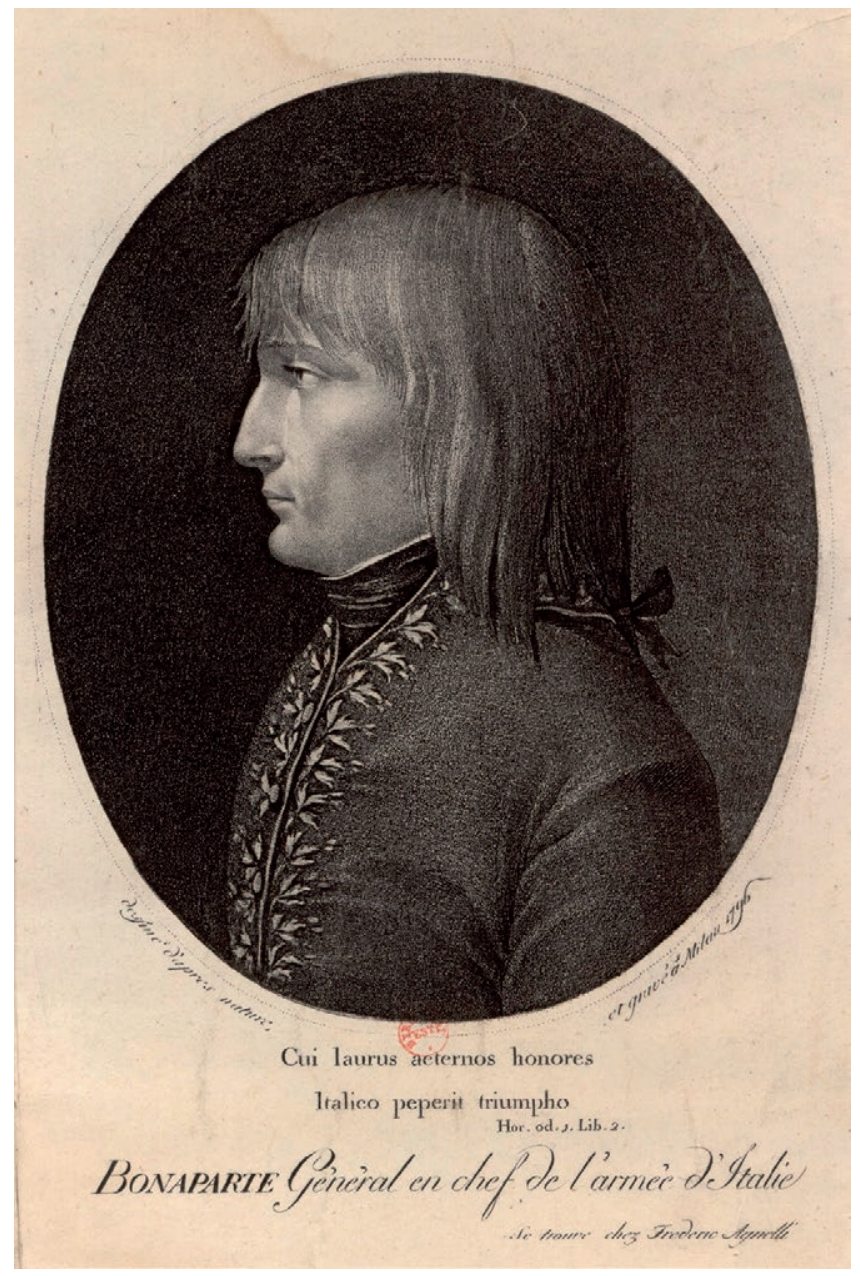

FIG. 2. - Portrait de Napoléon Bonaparte dessiné et gravé à Milan en 1796 alors qu'il était général en chef de l'Armée d'Italie (Frederic Agnelli, éditeur) (c) Bibliothèque nationale de France, cote QB-370 (50)-FT 4.

Bonaparte prit à Milan un arrêté pour sanctionner la ville de Vérone, lui imposant notamment de verser 120000 sequins. Larrêté prévoyait la confiscation des biens des condamnés jugés coupables d'avoir conspiré contre la République française, la saisie de l'argenterie dans les églises et les bâtiments publics, la réquisition de tous les chevaux et de la quantité nécessaire de cuir pour la confection de 40000 paires de chaussures et 2000 paires de bottes ainsi que des draps et du tissu pour produire 12000 costumes militaires (Daru 1821: 328, 329).

\section{ACTE 1}

SAISIE DE LA PREMIÈRE COLLECTION GAZOLA (MAi 1797) L'article 11 de l'arrêté de Bonaparte prévoyait en outre la confiscation de "tous les tableaux, des collections de plantes, de coquillages, etc. " qui appartenaient à la ville et aux particuliers. L'application de cette disposition fut confiée aux commissaires chargés de rechercher des objets d'art et de science. Ces commissaires, qui accompagnaient les troupes françaises, avaient en effet pour mission de rapporter en France des œuvres d'art, des livres et des spécimens d'histoire naturelle (Laporte 2008; Lacour 2009). Le ministre de la justice, Philippe-Antoine
Merlin (1754-1838), confirmait à Bonaparte la légitimité des confiscations dans une lettre en date du 11 ventôse an $\mathrm{V}\left(1 \mathrm{er}^{\mathrm{er}} \mathrm{mar} 1797\right)$ : "Les arts et les sciences réclament une foule d'objets précieux qu'ils ont créés et qui, longtemps détournés de leur véritable destination, doivent rentrer aujourd'hui dans le domaine de la liberté, source première de tout ce qu'ils offrent de beau et d'utile» (Pommier 1996). Ces actes commis à l'étranger n'étaient qu'une extension de pratiques similaires qui eurent lieu, dès 1792, sur le territoire français où d'innombrables bâtiments religieux et châteaux furent vidés de leurs objets d'art et de leurs cabinets d'histoire naturelle. Pour reprendre les arguments des promoteurs de ces spoliations, il s'agissait au nom de l' "idéal républicain» de confisquer aux ecclésiastiques et aux aristocrates «dilettantes» des objets qu'ils avaient accumulés pour leur seul plaisir et de les déposer dans des institutions publiques afin de les rendre utiles à l'instruction du citoyen éclairé. Sur les territoires occupés par l'armée républicaine, s'ajoutait à cette justification, le discours du juste dédommagement par les fruits de la victoire.

D'octobre 1794 à juin 1796, avant l'occupation de Vérone par les troupes françaises, Giovanni Battista Gazola avait accordé l'hospitalité au frère de Louis XVI, le futur roi Louis XVIII, qui était alors en exil (Gallas 1970; Gaudant 2011a). Cet attachement à la famille royale de France, rendit nécessairement Gazola suspect aux yeux des cadres de l'Armée d'Italie. Gazola fut d'abord incarcéré, avant d'être relâché, et le parc de sa maison saccagé, comme il le déclara en décembre 1814 dans une lettre à Louis XVIII (Annexe 1[doc. 60]). En application de l'arrêté de Bonaparte du 17 floréal an 5, la commission des sciences et des arts conduite par Claude-Louis Berthollet (1748-1822) pénétra dans le palais Gazola le 28 floréal an 5 (17 mai 1797). La collection de poissons et végétaux pétrifiés du Monte Bolca qui y était exposée fut jugée digne d'intérêt d'être transportée au "Muséum de Paris" (Nicolis 1907: 43, 44; Frigo \& Sorbini 1997; Gaudant 2011b). Ainsi s'achève l'histoire jusqu'alors connue de la confiscation de la collection Gazola.

\section{ACTE 2}

ReMords De BeRTHOLLET - PREMIÈRE INDEMNISATION (Juillet 1797-SEPTEMBRE 1797)

Alors que durant le mois de juin 1797, la collection Gazola était emballée dans des caisses en préparation de son transport, Claude-Louis Berthollet fut pris de remords. Arrêtons-nous un instant sur cette personnalité : Berthollet naquit à Talloires près d'Annecy dans le duché de Savoie qui faisait à l'époque partie du royaume de Sardaigne (Fig. 3). Il fit des études de médecine à l'université de Turin où il fut reçu docteur en 1768. Il partit à Paris et, grâce au soutien du premier médecin du duc d'Orléans, il fut nommé médecin de Madame de Montesson (1738-1806). Passionné par la chimie, il suivit les cours de Pierre Joseph Macquer (1718-1784) au Jardin des plantes, et ceux de Jean-Baptiste-Michel Bucquet (1746-1780) à la faculté de médecine de l'université de Paris. À partir de 1776, Berthollet publia de nombreux mémoires en chimie et fut reçu à l'Académie des sciences, à la place laissée vacante par la mort de Bucquet, en 1780. Il fut ensuite nommé directeur 


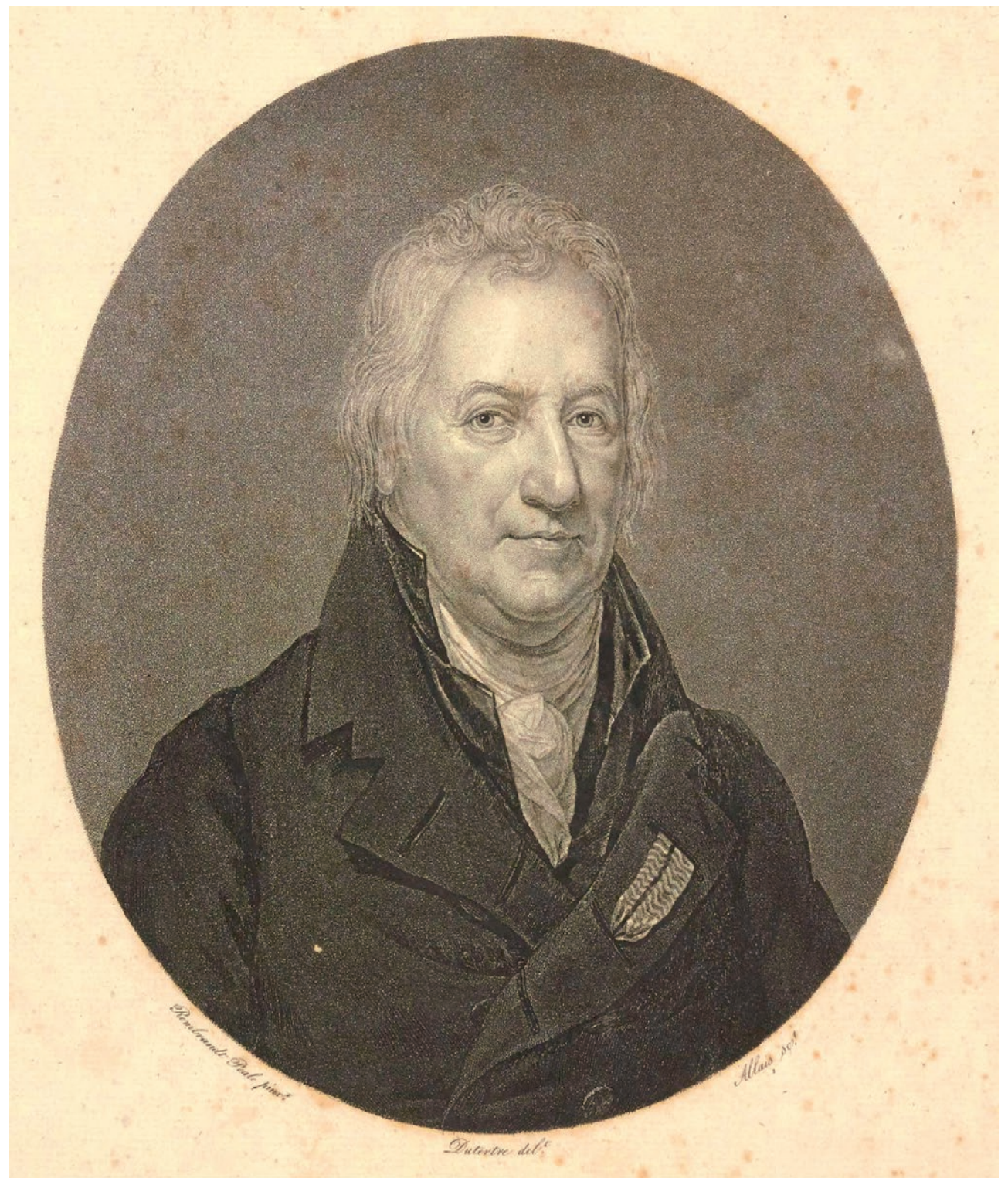

FIG. 3. - Claude-Louis Berthollet (9 décembre 1748, Talloires, Haute-Savoie - 6 novembre 1822, Arcueil). Portrait publié dans la Description de l'Égypte, Antiquités, Mémoires, tome 2 (Imprimerie royale, Paris, 1818), dessin d'André Dutertre (9 juin 1753, Paris - avril 1842, Paris), lithographié par Louis-Jean Allais (1762, Paris -27 août 1833, Paris) d'après une peinture de Rembrandt Peale (22 février 1778, comté de Bucks, Etats-Unis - 3 octobre 1860 , Philadelphie). Berthollet organisa la saisie de la collection Gazola à Vérone le 28 floréal an 5 (17 mai 1797). À sa demande, il fut décidé d’indemniser Gazola en juillet 1797.

des teintures à la manufacture royale des Gobelins en 1784, membre de la commission des monnaies en 1792 , membre de la commission d'agriculture en 1794 et, à partir de décembre 1794, professeur de chimie de la toute jeune École centrale des travaux publics, qui deviendra l'École polytechnique l'année suivante. Il suivit Napoléon Bonaparte durant les campagnes d'Italie et d'Égypte, comme commissaire des sciences et des arts. Après son retour en France, il occupa de hautes fonctions politiques, fut nommé membre du Sénat en 1799 et devint pair de France sous la Restauration.

En tant que scientifique, Berthollet avait un profond respect pour Gazola, qui avait amassé à grands frais une collection d'une si haute importance pour la géologie. Il faut dire qu'en acquérant les cabinets de Gian Giacomo
Dionisi et Vincenzo Bozza, Gazola avait contracté des rentes viagères d'un montant cumulé de 180 sequins. Privé de sa collection, il se retrouvait ainsi en position de continuer à payer un bien qui ne lui appartenait plus. L'arrêté du 17 floréal an 5 prévoyait le versement d'éventuelles indemnisations aux particuliers à qui l'on avait confisqué des collections d'objets d'art ou d'histoire naturelle (Daru 1821: 329). Les finances de l'Armée d'Italie étant exsangues, ces compensations devaient être prises sur les biens des condamnés ou sur d'autres biens saisis. Berthollet s'adressa à Napoléon Bonaparte en personne pour plaider la cause du "citoyen Gazola». Bonaparte proposa que lui fût octroyée la grande plaine de Prebiano, située à Villafranca près de Vérone. Il chargea le citoyen Haller d'en estimer la valeur et 


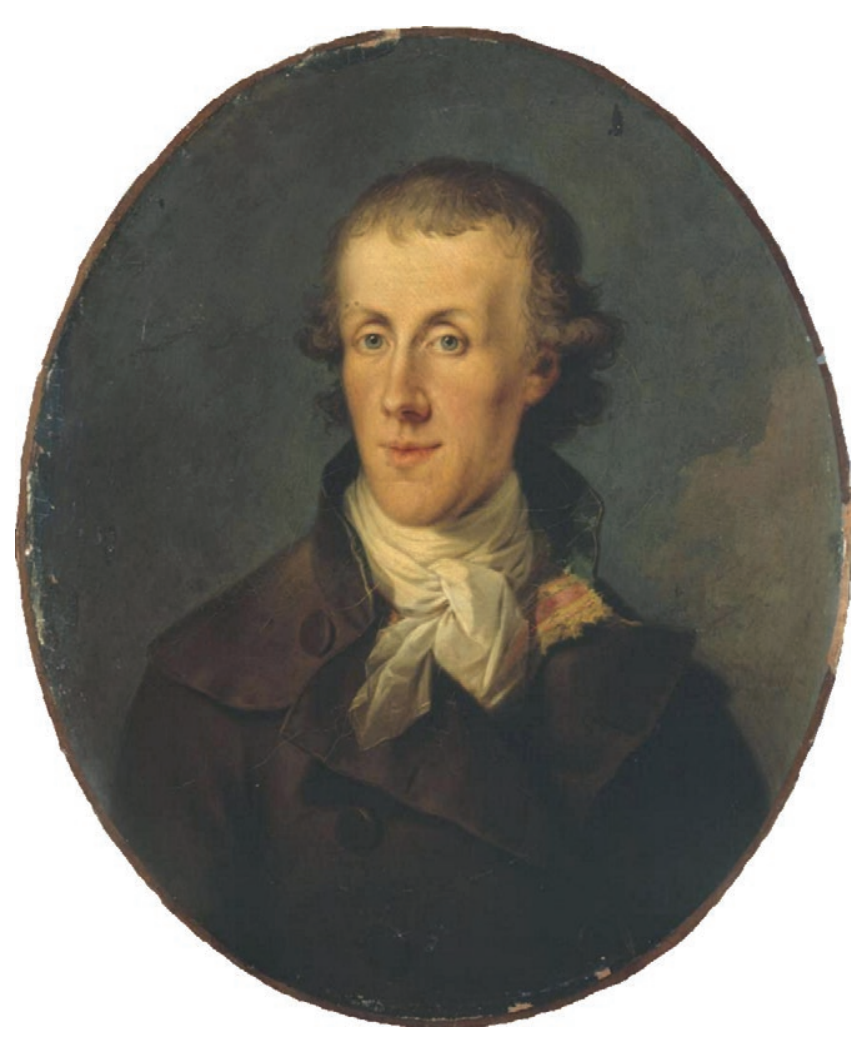

FIG. 4. - Emmanuel de Haller (1747-1833). Huile sur toile, École française, vers $1790,65 \times 54 \mathrm{~cm}$, ( ) Musée Carnavalet, Histoire de Paris, numéro d'inventaire P1215. Administrateur des contributions et finances d'Italie, il prit un arrêté le 26 thermidor an 5 (13 août 1797), approuvé par Napoléon Bonaparte, pour indemniser Gazola.

de prendre un arrêté pour officialiser cette indemnisation. Rodolphe Emmanuel de Haller, né en 1747, était issu d'une famille protestante de Berne en Suisse (Demougeot 1974) (Fig. 4). D'abord banquier à Amsterdam en 1768 puis à Paris à partir de 1777, il amassa une fortune considérable sans cesser d'avoir l'estime du pouvoir royal. Au début de la Révolution, Haller sentit le vent tourner et proposa ses services à l'administration. Lorsque la France rentra en guerre contre le Royaume de Sardaigne en 1793, Haller fut nommé régisseur des vivres de l'Armée d'Italie. Accusé de malversation, il partit se réfugier en Suisse en 1794, d'où il organisa sa défense. Réhabilité sous le Directoire, on fit à nouveau appel à ses services en 1796 pour gérer les finances de la campagne d'Italie. En août 1796, il fut nommé directeur de la monnaie de Milan et administrateur général des contributions et finances d'Italie sous les ordres du général Bonaparte. En 1799, il s'établit à Lausanne et remplit occasionnellement des missions diplomatiques pour les autorités de la République helvétique. Installé à Paris en 1816, il fonda en 1819 une banque qui fit faillite l'année suivante. Il mourut ruiné à San Benedetto Po, près de Mantoue, le 1er novembre 1833.

Haller chargea deux de ses agents, les citoyens Rochejean et Boulanger, de prendre des renseignements sur la prairie de Prebiano "que le Général en Chef a accordée en indemnité au Citn Gazzola [sic] sur la demande du Citn Bertholet [sic]".
Ils rendirent leur rapport le 2 thermidor an 5 (20 juillet 1797) (Annexe 1[doc. 1]). Composée de 1075 champs, cette prairie produisait annuellement une quantité de foin pour une valeur de 12000 livres de Venise, équivalente à 6000 francs de l'époque (Annexe 1[doc. 3]). Ce bien avait été mis à la disposition de la municipalité de Villafranca par Vérone, avec obligation en échange d'entretenir un ancien château. Ce dernier ayant été détruit, la rente que fournissait la prairie était en quelque sorte dégagée de toute servitude. Ne voyant aucun obstacle à céder ces terres à Gazola, Haller prit un arrêté en date du 26 thermidor an 5 (13 août 1797) "à l'effet d'obtenir une indemnité en faveur du Citen Jn Baptiste Gazzola de Verone pour la collection complette [sic] de son cabinet d'histoire naturelle composée des poissons plantes pétrifiées qu'il a cédé à la Republique française, pour augmenter les collections du Museum national d'histoire naturelle de Paris» (Annexe 1[doc. 2]). Bonaparte signa en personne cet arrêté (Fig. 5). Malgré cela, les choses ne se déroulèrent pas aussi simplement et la commune de Vérone, spoliée de son bien, s'opposa fermement à cette donation. Cette première tentative pour indemniser Gazola était dans une impasse et Berthollet écrivit à nouveau au général Bonaparte (Gaullieur 1856: 663):

Citoyen Général, permettez-moi de vous parler encore de l'indemnité que j'ai réclamée auprès de vous pour le citoyen Gazzola auquel j'ai enlevé pour notre Museum une inestimable collection de poissons pétrifiés.

S'il s'agissait des intérêts de ma fortune, je ne vous importunerais pas; mais c'est ma tranquillité morale que je vous demande. C'est de me mettre à l'abri des reproches que me feraient tous ceux qui s'intéressent aux sciences que je vous supplie.

Le citoyen Gazzola a payé de fortes contributions, comme les autres Véronais, en argent, chevaux et voitures, et outre les grandes dépenses qu'il a faites pour sa collection, il se trouve chargé de deux rentes viagères.

Je vous ai proposé de lui assurer une prairie qui n'est possédée qu'abusivement, et que la municipalité de Vérone vient de ressaisir. Le citoyen Haller a besoin de votre renvoi pour opérer cette cession. Si vous n'y apercevez pas d'inconvénient, ayez la bonté de le prononcer. Il sera pour moi un bienfait particulier.

Berthollet

Cette lettre prouve l'importance que Berthollet attachait à l'indemnisation de Gazola pour «sa tranquilité morale» et pour "se mettre à l'abri des reproches" que ne manqueraient pas de lui faire "tous ceux qui s'intéressent aux sciences". Bonaparte, qui avait déjà donné son accord une première fois, accepta cette seconde requête et la fit suivre à Haller "pour faire des dispositions en conséquence» (Gaullieur 1856: 663). Comme il semblait difficile de donner à Gazola un droit sur des terres qui appartenaient à la République de Venise, Haller se tourna vers des biens qui dépendaient de l'état de Mantouan, sous domination française depuis la capitulation de Mantoue, le 2 février 1797. L'accord entre la 
Les conditions d'acquisition de la collection Gazola par le Muséum national d'Histoire naturelle \}

Xiberté

Bepuobligue if rancaifet

Eqalite.

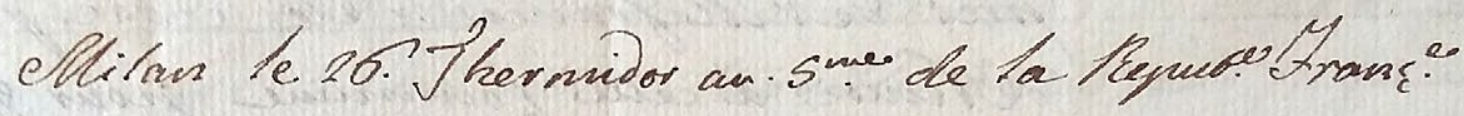
une, et indirifible

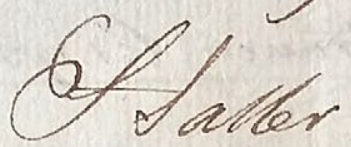

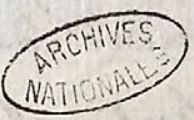

Coninifratear Des fontributions el Hinance: 20Ytate.

$\int_{\mathbb{1} a}$ Lenume facte por le fommisaire bu qowernement francais a' a vecherche def objets de sciencefe et etrts en thacie, a tiepers.

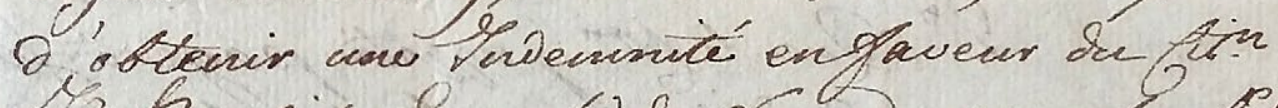

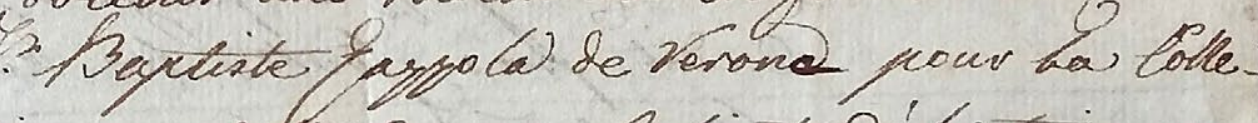
dion complette de con fabinet o histocie na:

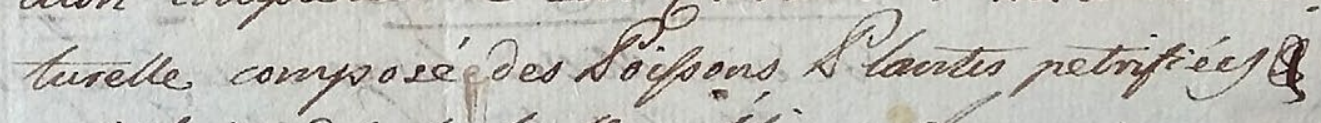

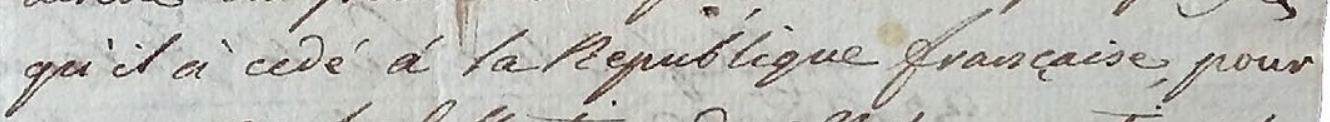
angmenter lesfoftections an enciferm notionar ofistoine naturelle de Sarid.

A I Etat propuite par te dit Esypta des depensy que cette collection hie a cowté depuig quibue ans, et les prix qu' it a paye pour remis Siver. ses collections la fiemula; ainsi que les ventes viagiéres D'environ 180. Sequins, qui r paye en cone a Sivers Amateuss zowt bes fabiness bi

FIG. 5. - Première page de l'arrêt du Citoyen Haller (Rodolphe Emmanuel de Haller), alors administrateur des contributions et finances d'Italie, en date du 26 thermidor an 5 (13 août 1797) par lequel la République française octroie le pré de Prebiano près de Villafranca dans le Véronais au "Citoyen Jean Baptiste Gazzola [sic]" en indemnisation de la collection de poissons et de plantes pétrifiés qu'il a "cédée" à la République française (voir Annexe 1[doc. 2] pour la transcription complète). (c) Archives nationales (F/17/1537, sous-dossier Gazola).

GEODIVERSITAS • $2019 \cdot 41$ (2)

17 
- Brignon A.

ont été cềés.

Gonsîeraut que ta Dravie momé Arebcano

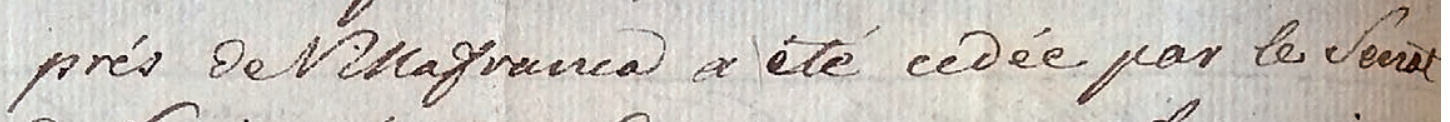

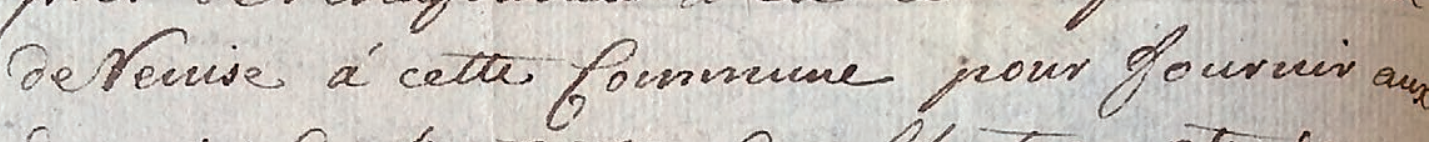
iepertel de fentretien an bhatcan et des

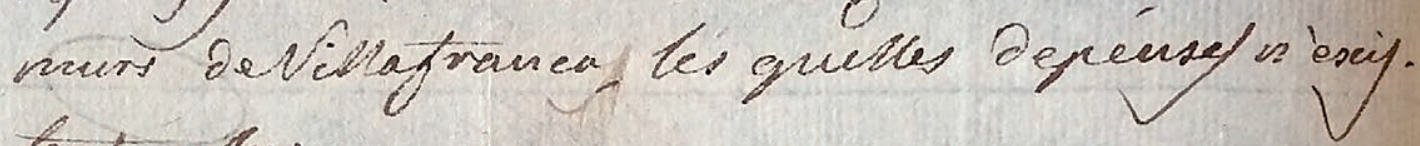
tut plus.

Quréte

- Pue ledit prér nommé Drebiano est domé et concedé par da Eyperbliguêt tran caise en

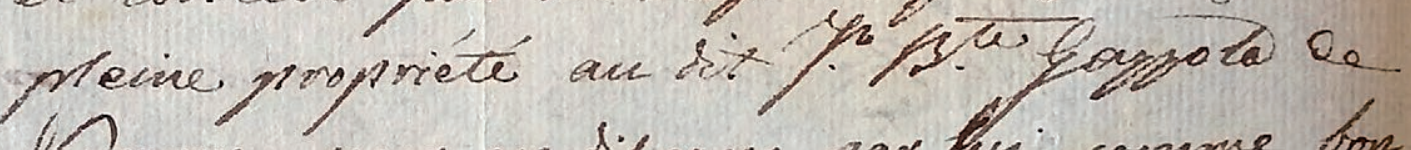
Verome pour en diproses par bu comme bon

2. Que le Gir Gassota pazena fur la proinet Dudit sres towies les munies quathefouts hives det Hance de nexte piour majutal

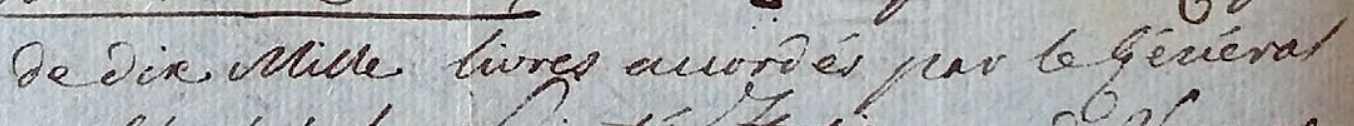

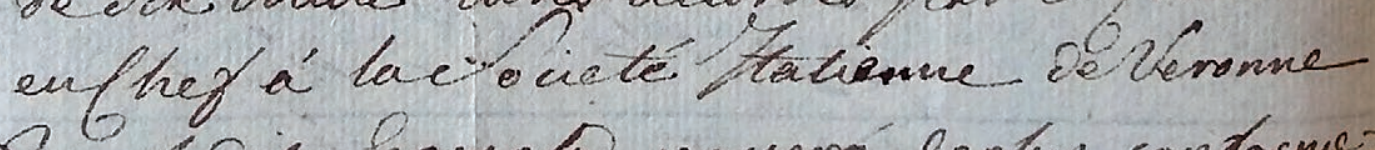

3: Pue kodt faysolo payena deptus cenforme:

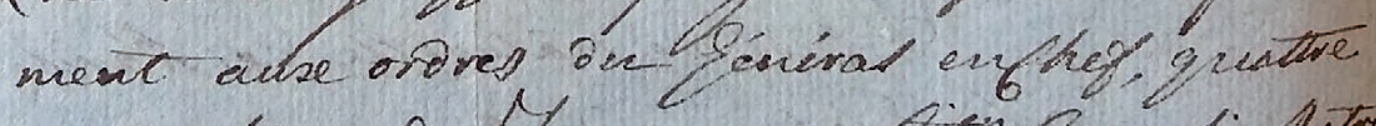
nilie hives dectrance, ace fitin Gaysoli dstronome de Vevone, pour enoemité qui bur sit Dre, et qui bi a sté allonée

Ce. fowevnement fentral Dec Veromsais reste chargé De faire nethe a's executson be présect terrét, et upcialement de faire

FIG. 5. - Suite (deuxième page de l'arrêt).

18

GEODIVERSITAS • 2019 • 41 (2) 


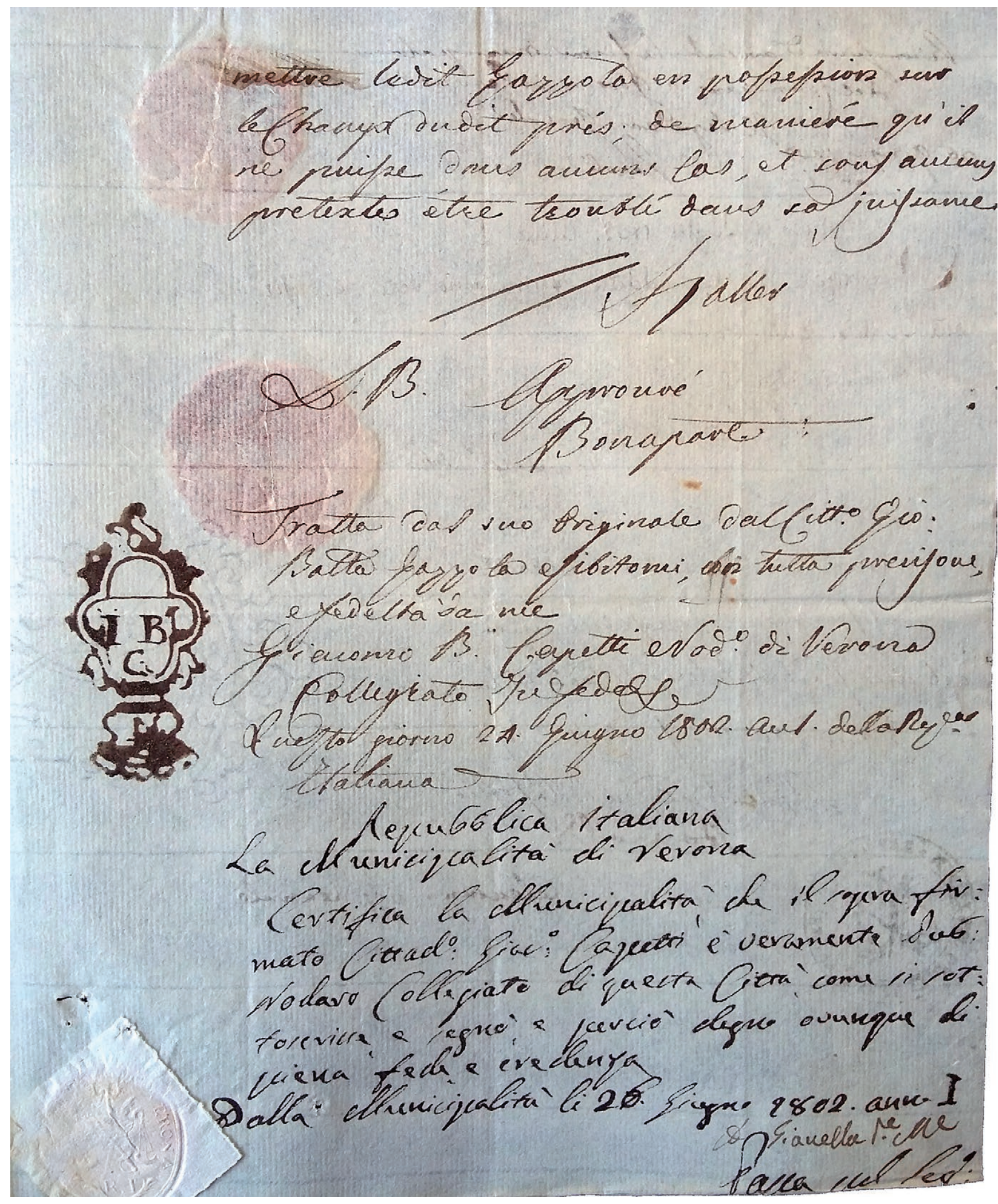

FIG. 5. - Suite (troisième page de l'arrêt). À noter la mention «approuvé Bonaparte », qui était alors général en chef de l'Armée d'Italie, et le tampon de Gazola avec ses initiales en latin «/B $G »$.

République française et l'administration de l'état de Mantouan visant à indemniser Gazola fut signé le 8 vendémiaire an 6 (29 septembre 1797) (Fig. 6). Le document stipule que "la detta amministrazione separerà di beni fondi, che le sono ceduti una porzione qualunque producente tre milla lire di Milano, da rimettersi in tutta proprietò del Citto [Cittadino] 




FIG. 6. - Copie en italien de la transaction passée le 8 vendémiaire an 6 (29 septembre 1797) entre la République française et l'administration de l'état de Mantouan pour indemniser Gazola. (C) Archives nationales (F/17/1537, sous-dossier Gazola).

Gazzola [sic] per indemnizzato del Cabinetto di pesci petrificati ed altre cose ch'egli ha ceduto alla Repubba [Repubblica] Francese» [ladite administration séparera sur les biens qui lui sont cédés une portion quelconque produisant 3000 livres de Milan, pour être remise en toute propriété au Citoyen Gazola, pour l'indemniser du cabinet de poissons pétrifiés et autres objets qu'il a cédés à la République française]. Cet accord rentra en application et Gazola toucha donc dès le 


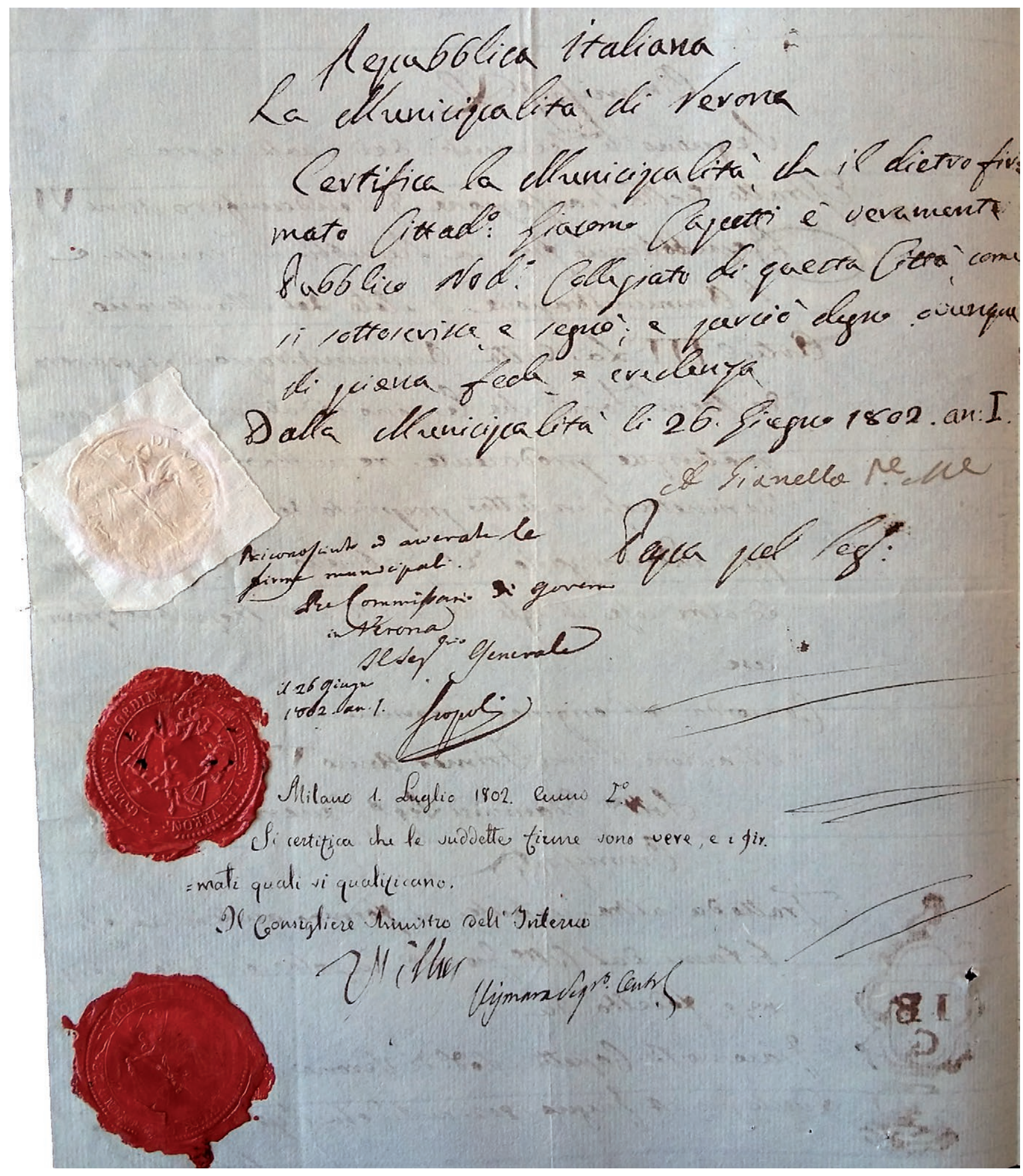

FIG. 6. - Suite (verso de la page précédente).

mois de septembre 1797 une rente annuelle de 3000 livres de Milan, soit environ 2300 francs de l'époque. D'après le témoignage de Bartolomeo Giuliari, cette rente provenait de deux biens, nommés "Bel-Brol» et "Collarina», représentant un capital cumulé de 100000 lires italiennes (Giuliari 1871: 173).

\section{ACTE 3}

L'ARRIVÉE TRIOMPHALE à PARIS De LA COLLECTION GAZOla 1 (JUILleT-SePTEMBRE 1798)

Ce qui était une confiscation devenait donc une transaction, même si Giovanni Battista Gazola eut certainement peu de marge de manœuvre pour négocier. Une des conséquences 
de la présence française dans le nord de l'Italie fut la proclamation de la République ligurienne, le 6 juin 1797, et de la République cisalpine, le 27 juin 1797. Pour dédommager l'empire autrichien et tenter d'assurer la pérennité de ces républiques nouvellement créés, la Vénétie fut cédée à l'Autriche en application du traité de Campo-Formio signé le 26 vendémiaire an 6 (18 octobre 1797) entre Napoléon Bonaparte et le représentant de l'empereur d'Autriche. Disparaissait ainsi la République de Venise, et la ville de Vérone rejoignait l'empire autrichien (Fig. 7). Compte tenu de ces conditions géopolitiques, Gazola dut, dans un premier temps, se contenter de ce qui lui avait été octroyé par Bonaparte dans l'arrêté du 8 vendémiaire an 6 .

Cette première collection (Gazola 1) mit plus d'un an pour arriver à Paris. Les 9 et 10 thermidor an 6 (27-28 juillet 1798), à l'occasion des "fêtes de la Liberté», fut organisée par le ministre de l'Intérieur, Nicolas François de Neufchâteau (1750-1828), l'entrée triomphale à Paris des objets des sciences et d'arts recueillis en Italie (François de Neufchâteau 1798; Pagès 1800: 203-208) (Fig. 8). Le premier jour, «à neuf heures du matin tous les citoyens, invités à composer le cortège qui devoit accompagner les monumens antiques et autres fruits de nos conquêtes, se sont réunis sur la rive gauche de la Seine, près le muséum d'histoire naturelle. Les chars qui portoient ces monumens, étoient ornés de trophées, de guirlandes, et d'inscriptions analogues. La marche du cortège étoit ouverte par un détachement de cavalerie, et par un corps de musique militaire. Le cortège et les chars formoient trois grandes divisions». En avant de la première division, "on portoit une bannière, sur laquelle on lisoit: Histoire naturelle. Venoient ensuite les professeurs administrateurs du muséum d'histoire naturelle et les élèves et amateurs auxquels on avoit distribué des cartes d'admission pour le cortège». Un des chars était entièrement dédié aux «pétrifications de Vérone» et portait l'inscription "des antiquités du globe». Le second jour des festivités, le directoire exécutif, les autorités admnistratives et les ambassadeurs des puissances étrangères prirent place au Champ-de-Mars. "Les commissaires qui avoient été envoyés en Italie pour le choix et l'envoi des monumens, traversèrent avec les chars l'arène jusqu'à l'autel de la patrie». Une mongolfière ornée de guirlandes portait "dans les airs les attributs de la liberté et des arts, et des drapeaux aux trois couleurs". Et les commentateurs de l'époque de conclure: "quelle riche moisson pour les beaux arts et les sciences! Quelle est grande la nation qui a fait de telles conquêtes! Quelles reconnoissance, que de palmes ne devons-nous pas aux généreux guerriers qui ont élevé la République française à ce haut degré de splendeur et de gloire!». Lévènement fut immortalisé sur une gravure publiée dans les Tableaux historiques de la Révolution française (Auber 1802: vol. 3, pl. 136; Lacour 2014: 131).

C'est seulement à partir du 2 vendémiaire an 7 (23 septembre 1798), qu'il fut procédé à l'ouverture des caisses qui contenaient la collection Gazola (Gaudant 2011a: 92 ; 2011b: 642). On apprend dans des rapports du ministère de l'Intérieur datant de 1803, que la "collection amenée à Paris» avait «beaucoup souffert faute d'avoir pris les précautions nécessaires» (Annexe 1[doc. 6]) et que toutes les caisses arrivèrent brisées (Annexe 1[doc. 15]). Bartolomeo Giuliari avait fait dessiner une grande partie des pièces de la collection Gazola avant leur transfert à Paris. Il put ainsi poursuivre la publication de l'Ittiolitologia Veronese. Gazola, quant à lui, continuait de faire activement fouiller la Pesciara. Il acheta également le cabinet du comte Ignazio Ronconi, si bien qu'il reconstitua en quelques années une nouvelle collection de pétrifications du Monte Bolca.

\section{ACTE 4}

\section{Un cadeau pour le Premier Consul et}

MISE EN PLACE D'UNE SECONDE INDEMNISATION

(OCTOBRE 1802-AVRIL 1803)

La paix entre la France et l'Autriche ne fut que de courte durée. En 1799, profitant de l'absence de Napoléon Bonaparte bloqué en Égypte, les Autrichiens appuyés par les Russes lancèrent une offensive dans le but de reconquérir les possessions françaises en Italie. Alors que la situation était de plus en plus difficile pour l'armée française, Bonaparte, rentré d'Égypte, mena le coup d'État du 18 Brumaire (9 novembre 1799) et se fit nommer Premier Consul. À la tête de l'armée, Bonaparte parvint à renverser la situation en Italie, notamment après la bataille de Marengo, le 14 juin 1800. Il rentra ensuite à Paris laissant ses généraux régler la guerre en Italie, qui s'acheva par la victoire de la France et le traité de Lunéville signé le 20 pluviôse an 9 (9 février 1801). Le traité instaura, entre autres, un équilibre en Italie entre la France et l'Autriche. Cette dernière dut reconnaître la République ligurienne et la République cisalpine sous protection française. En échange, la maison d'Autriche se vit confirmer les possessions de la Vénétie orientale, l'Istrie, la Dalmatie et les bouches du Cattaro. L'Adige matérialisait la frontière entre l'Autriche et la République cisalpine, si bien que la ville de Vérone fut coupée en deux. Le 26 janvier 1802, la République cisalpine fut renommée République italienne et Bonaparte s'en fit proclamer président. Le palais de Gazola situé au 2 Piazzetta Chiavica se trouvait "côté français " et n'était qu'à cinquante mètres de la frontière autrichienne et de Véronette, comme les Français appelaient la partie autrichienne de Vérone, sur la rive gauche de l'Adige.

Après cinq ans de tumultes militaires et d'instabilités géopolitiques qui avaient secoués Vérone, Giovanni Battista Gazola jugea la situation enfin propice pour faire savoir au gouvernement français qu'il estimait avoir été lésé lors de la mise en place de sa première indemnisation. L'arrêté du 3 août 1797 prévoyait qu’il touchât une rente annuelle équivalente à 6000 francs. Cet arrangement n'ayant pu se concrétiser, on lui avait donné un autre bien qui ne produisait qu'environ 2300 francs, soit un manque à gagner de 3700 francs par an. Gazola réclamait donc d'être dédommagé de cette différence. Il envoya sa pétition dans le courant de l'année 1802 à Talleyrand (1754-1838), alors ministre des Relations extérieures du gouvernement français, qui jugea "que cette demande lui avait paru peu fondée» (Annexe 1[doc. 3]). Pour Talleyrand, le second arrangement annulait les dispositions du premier. Gazola n'était selon lui "plus en droit de s'en prévaloir». "Tout a été terminé, consommé par les derniers accords par leur exécution». Gazola se tourna alors vers son ministre des Relations 

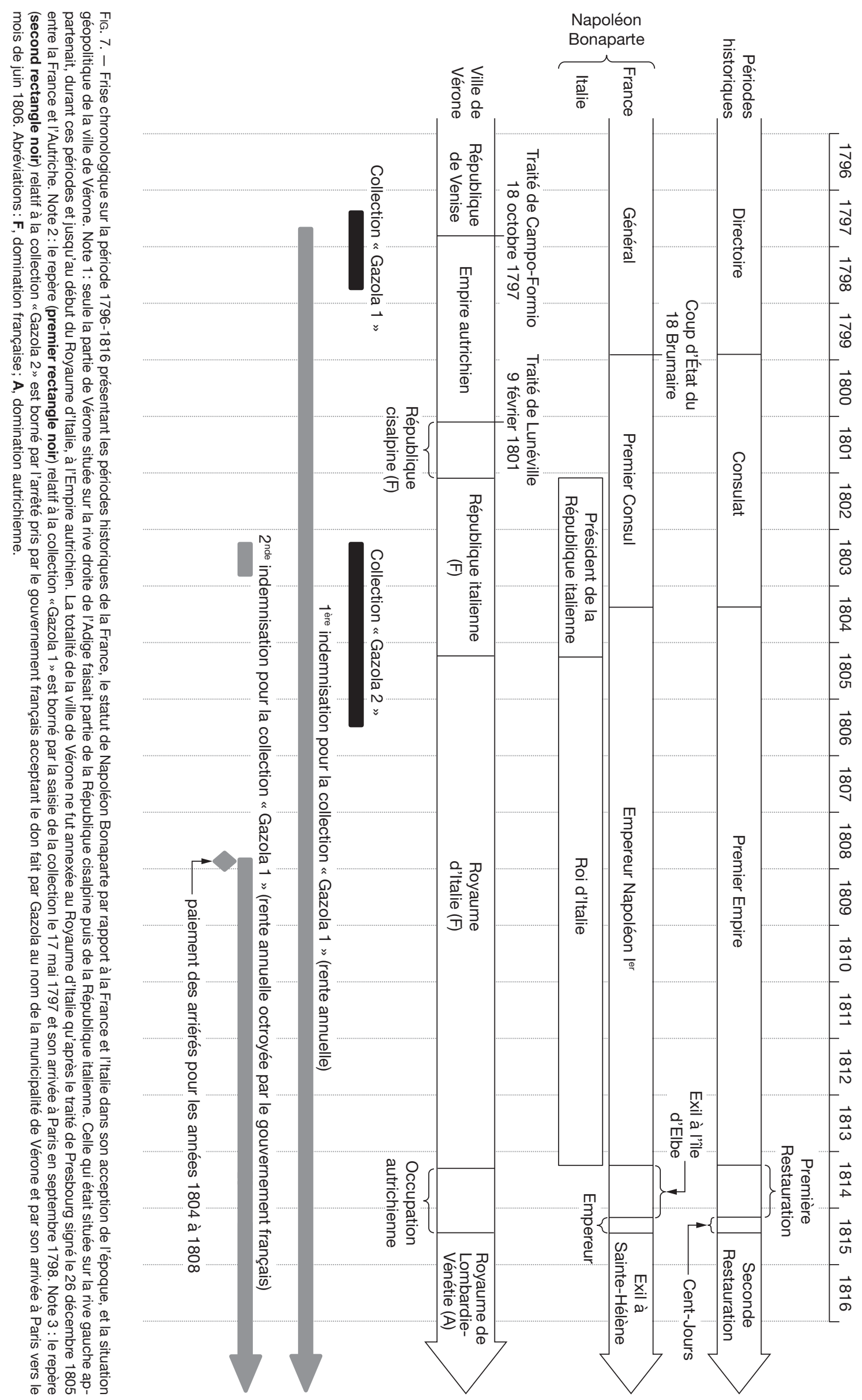

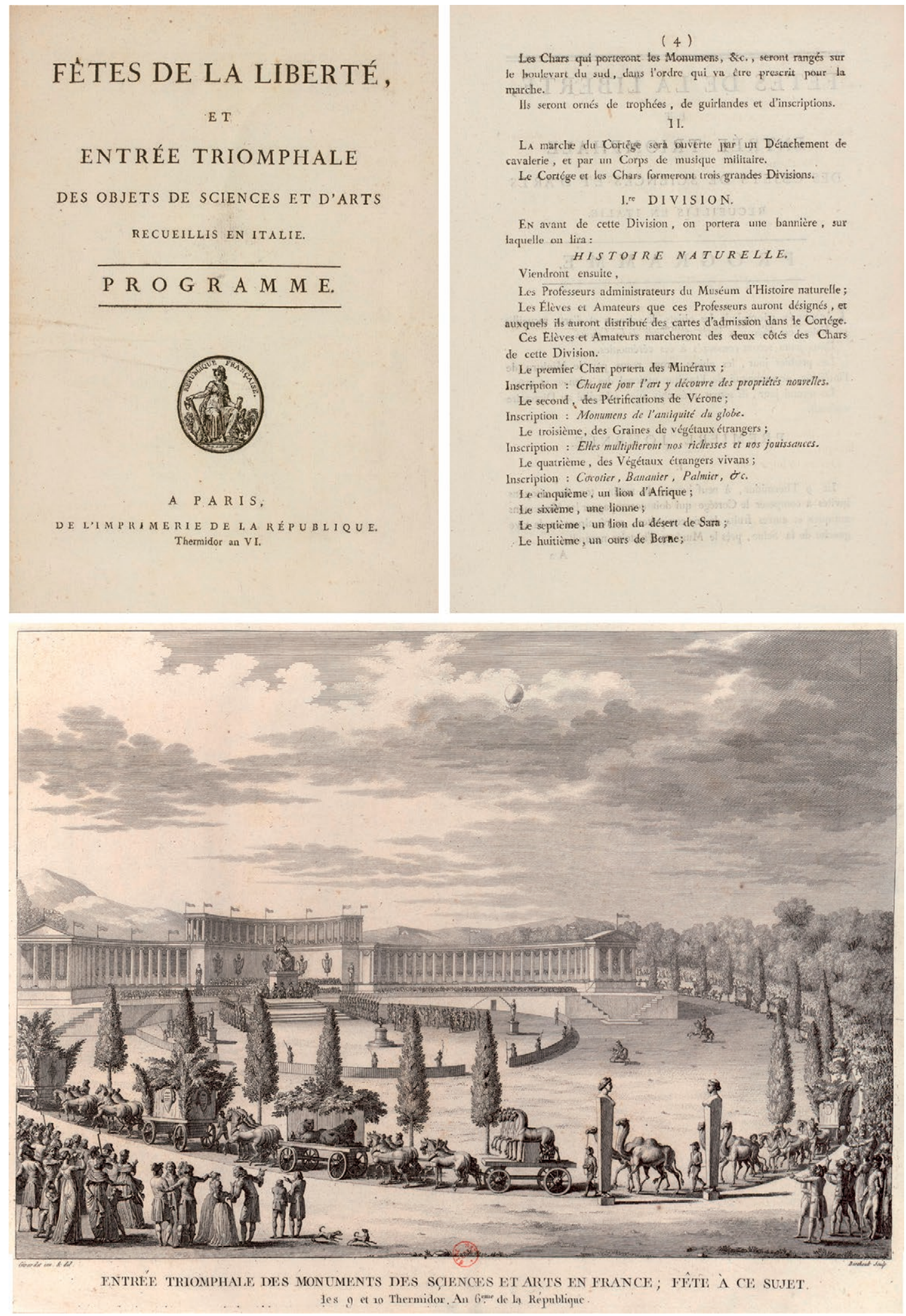

FiG. 8. - Programme des fêtes de la Liberté du 9 et 10 thermidor an 6 (27-28 juillet 1798), au cours desquelles les objets de sciences et d'arts recueillis en Italie firent leur entrée triomphale à Paris. En haut à gauche : page de titre du programme officiel signé par le ministre de l'Intérieur, Nicolas François de Neufchâteau (1798). En haut à droite: page du programme relative au défilé de la division d'«Histoire naturelle»; le deuxième char était dédié aux "Pétrifications de Vérone" autrement dit, en grande partie, à la collection Gazola. En bas, gravure de l'évènement (Auber 1802: vol. 3, pl. 136). On peut y voir des lions en cage, des dromadaires et, au centre, les quatre statues des chevaux de Saint-Marc rendus à Venise à 1815 après la chute de l'Empire. Le char dédié à la collection Gazola était en avant du cortège. 




FIG. 9. - Ferdinando Marescalchi (26 février 1754, Bologne - 1er octobre 1816, Milan) par le peintre d'origine autrichienne, Ludwig Guttenbrunn (1750-1819), huile sur toile, $1813,114 \times 101 \mathrm{~cm}$, châteaux de Versailles et de Trianon, numéro d'inventaire MV4790. Photo @ RMN-Grand Palais (Château de Versailles)/Franck Raux. 


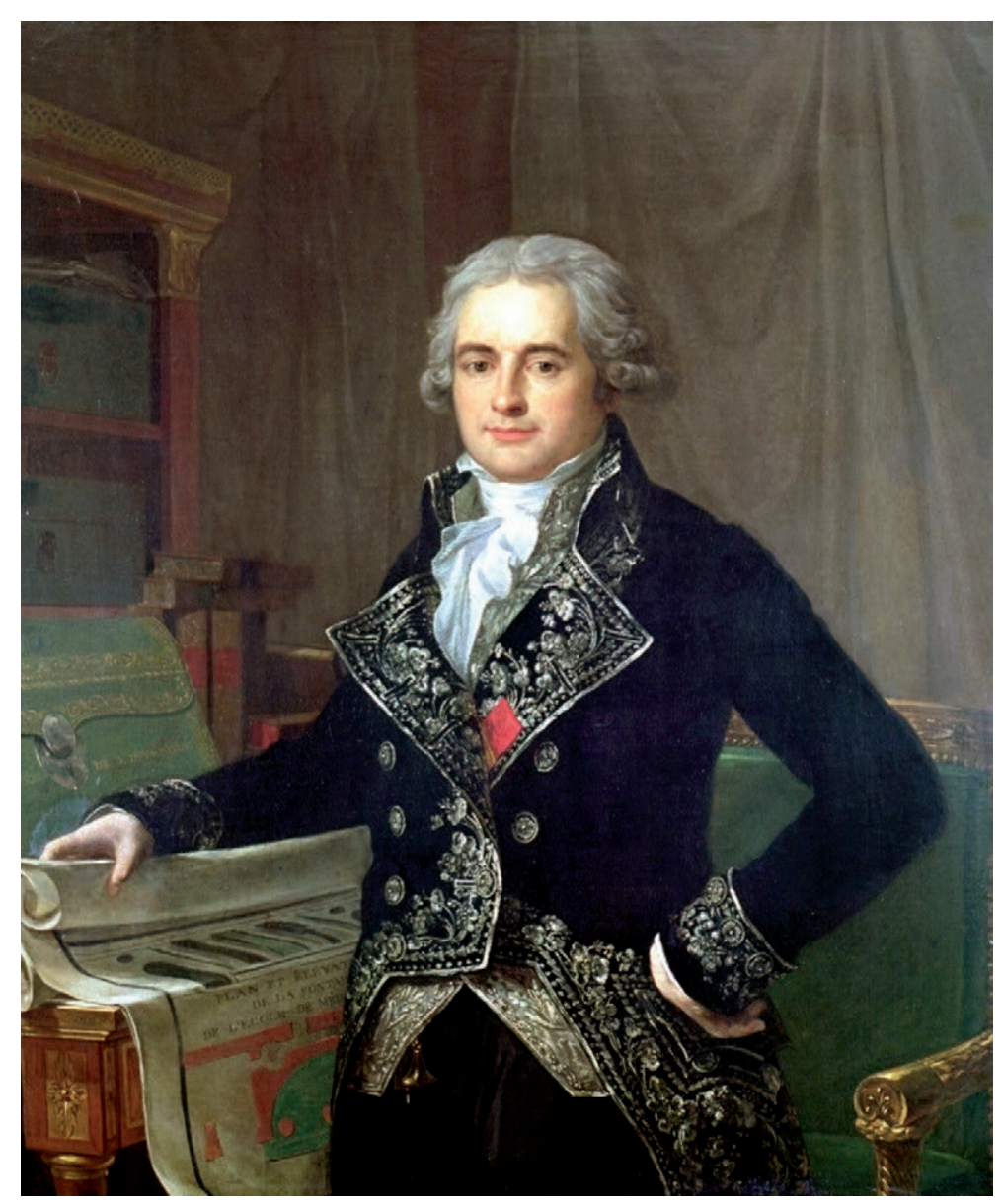

FIG. 10. - Jean-Antoine Chaptal (4 juin 1756, Nojaret, Lozère - 29 juillet 1832, Paris) par Anicet Charles Gabriel Lemonnier (6 juin 1743 , Rouen - 17 août 1824, Paris), huile sur toile, $107 \times 88$ cm (Tuilier 1989: 127, 128), numéro d'inventaire 89.01.0082, @ Musée de l'Histoire de la Médecine, Université Paris-Descartes.

extérieures, Ferdinando Marescalchi (1754-1816), en le suppliant de transmettre sa réclamation au Président de la République italienne, autrement dit au Premier Consul de la France, Napoléon Bonaparte en personne. Noble originaire de Bologne, Marescalchi avait pris ouvertement position en faveur des Français lors de leur arrivée en Italie. Ayant gagné la confiance de Bonaparte, il occupa plusieurs postes importants dans les Républiques cispadane et cisalpine (Fig. 9). Il fut nommé ministre des Relations extérieures en 1802 quand fut créée la République italienne. Il garda son poste jusqu’à la chute du Royaume d'Italie en 1814. Marescalchi fut toujours un allier de poids pour Gazola. Il se montra donc plus compréhensif que Talleyrand et s'étonnait que le second arrangement ne permît pas de dédommager Gazola du montant fixé par le premier arrêté. Il notait que le collectionneur véronais n'avait pas formellement accepté les termes du dernier accord et qu'il ne s'était donc pas engagé à renoncer "à toute plus ample prétention" (Annexe 1[doc. 3]). Le dossier, accompagné de la pétition signée par Gazola, fut transmis au Premier Consul. Une des phrases de la pétition rédigée à la troisième personne résume son contenu: "L'objet de sa demande, était le complément de payement d'une collection de poissons, et de plantes pétrifiées, qu'il a cédé en l'an 5 au Gouvernement Français» (Annexe 1[doc. 4]). Gazola suggérait d'être indemnisé par le versement d'un capital fixe de 64000 francs auquel il ajoutait 24000 francs d'arrérages correspondant à la somme non perçue de 3700 francs par an sur six ans. Les arrérages s'élevaient en réalité à 22200 francs et non 24000 francs. Quoi qu'il en soit, la somme totale que sollicitait Gazola était évaluée à 86500 francs, d'après le ministère de l'Intérieur dans son rapport au gouvernement (Annexe 1[doc. 7]). Dès le mois d'octobre 1802, Napoléon Bonaparte accepta de considérer cette requête et ordonna à son ministre de l'Intérieur, Jean-Antoine Chaptal, d'instruire le dossier (Fig. 10). Né le 3 juin 1756 à Nojaret, en Lozère, Chaptal étudia la médecine à Montpellier jusqu'en 1777, puis la chimie à Paris (Gueniffey 2011). De retour à Montpellier, en 1780, il occupa la chaire de chimie de l'université. Ses recherches en chimie appliquées à la vinification le rendirent célèbre. Pendant la période révolutionnaire, il dirigea la production de poudre de guerre et de salpêtre. Sous le Consulat, Napoléon Bonaparte lui confia le ministère de l'Intérieur. Il occupa cette responsabilité du 16 brumaire an 9 (7 novembre 1800) au 19 thermidor an 12 (7 août 1804). Chaptal réorganisa en profondeur l'instruction publique et contribua à la vulgarisation de la chimie et à son application à l'industrie et aux arts.

Vers la fin de l'année 1802, Giovanni Battista Gazola fit le voyage à Paris afin de défendre ses intérêts. L'obtention d'une 


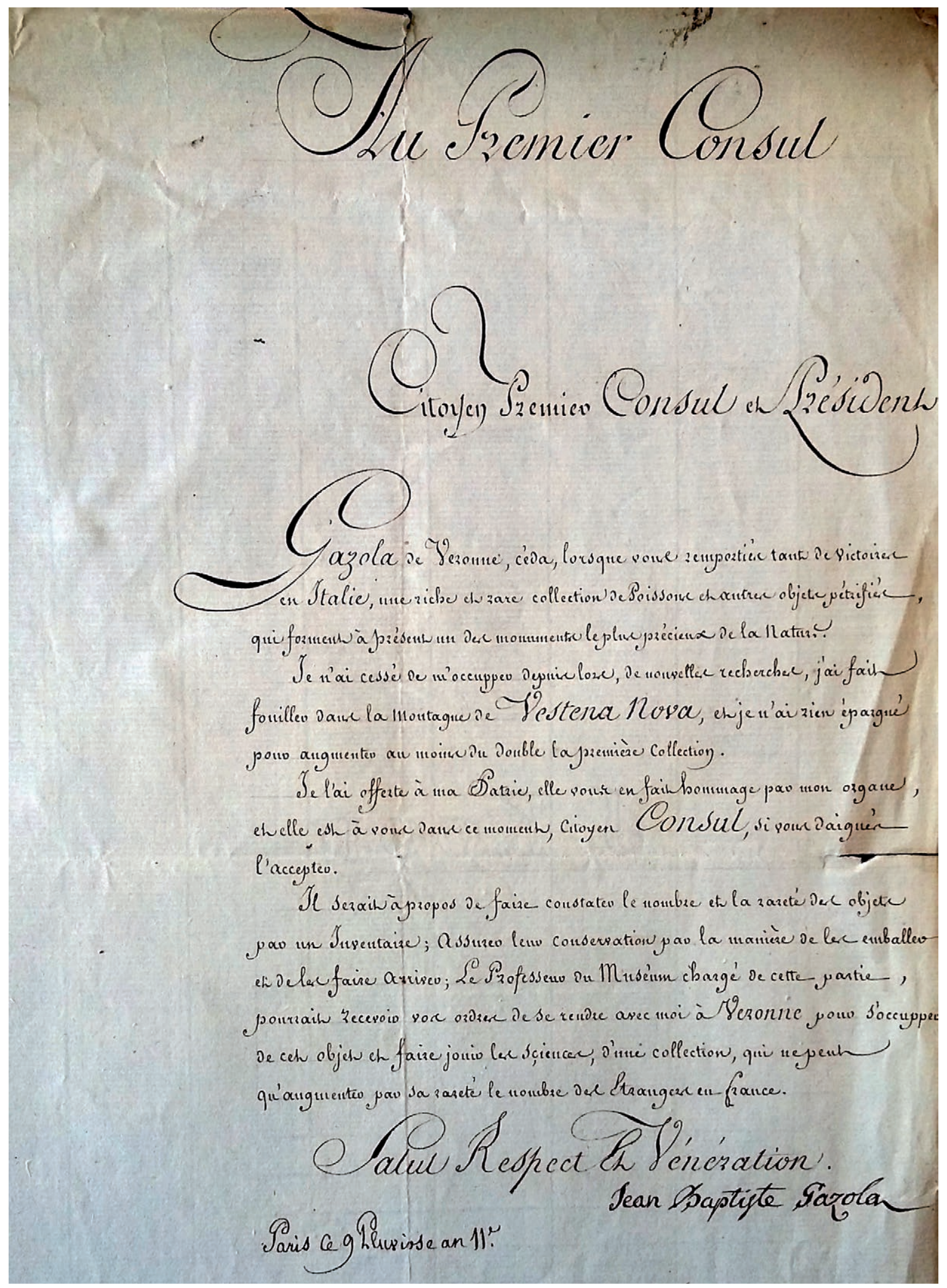

FIG. 11. - Lettre de Giovanni Battista Gazola à Napoléon Bonaparte, Premier Consul de la République française et Président de la République italienne, en date du 9 pluviose an 11 (29 janvier 1803). Il propose de lui offrir, au nom de la ville de Vérone, une seconde collection de poissons du Monte Bolca (voir Annexe 1[doc. 5] pour la transcription complète). (C) Archives nationales (F/17/1537, sous-dossier Gazola).

seconde indemnisation n'était pas sa seule motivation. Il avait également quelques faveurs à demander au Premier Consul au nom de la ville de Vérone et était accompagné dans cette mission par le député véronnais Gaetano Pinali (1759-1846). Les professeurs du Muséum national d'Histoire naturelle profitèrent de la venue de Gazola pour le convier à participer à 


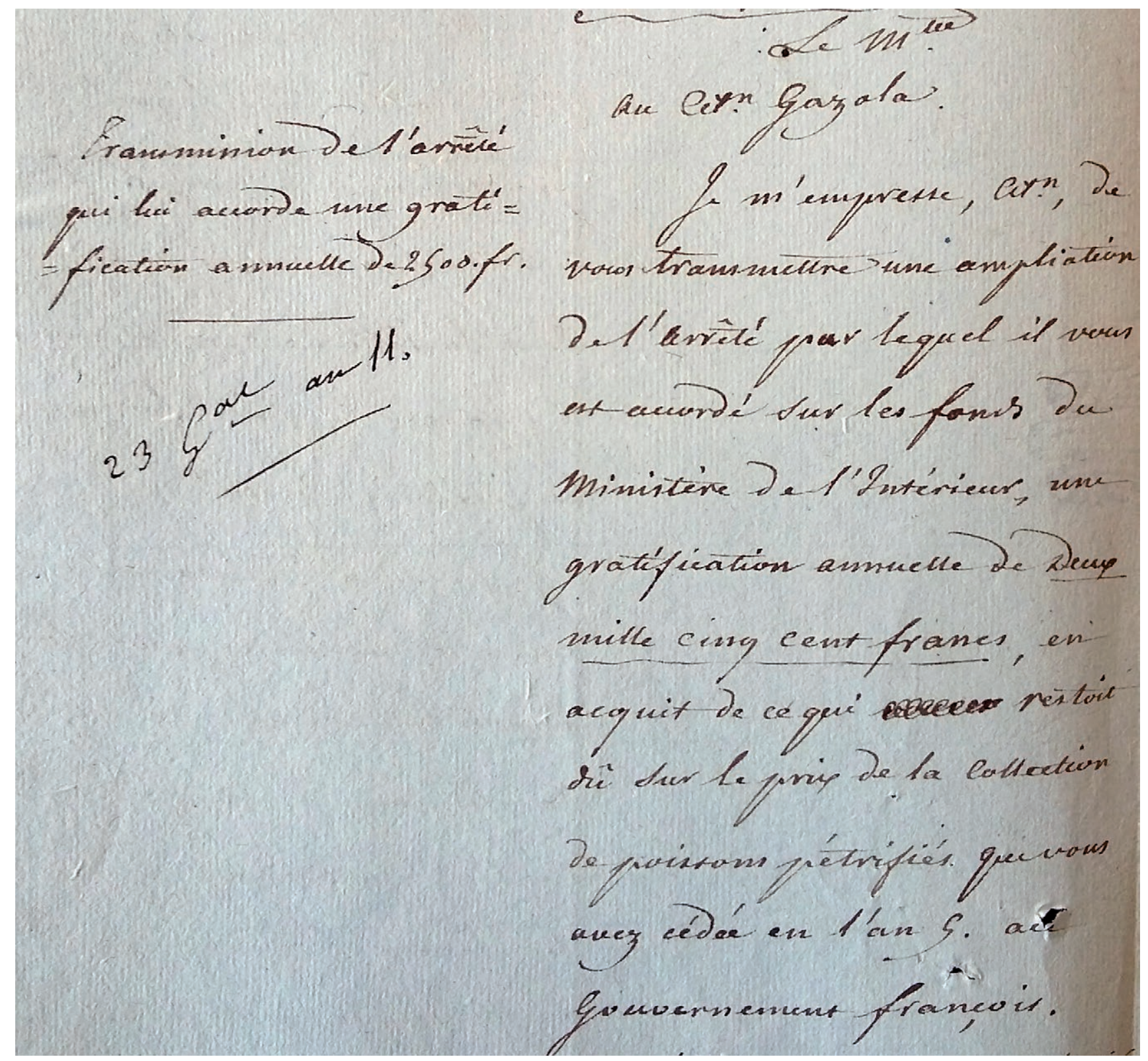

FIG. 12. - Copie d'une lettre de Jean-Antoine Chaptal, ministre de l'Intérieur, à Gazola en date du 23 germinal an 11 (13 avril 1803) lui annonçant une gratification annuelle de 2500 francs pour la collection de poissons fossiles cédée en 1797 au gouvernement français (voir Annexe 1[doc. 14] pour la transcription complète). (c) Archives nationales (F/17/1537, sous-dossier Gazola).

leur assemblée du 13 nivôse an 11 (3 janvier 1803) (Gaudant 2011b). À cette occasion, Gazola leur fit voir quelques fossiles qu'il avait apportés avec lui et notamment "une superbe collection de Poissons pétrifiés» disséminés dans des "feuillets réunis dans une bouëte souvrant en trois parties".

Giovanni Battista Gazola proposa d'offrir au nom de sa "Patrie» une seconde collection de pétrifications du Monte Bolca, pour compléter celle qui était conservée au Muséum national d'Histoire naturelle (Annexe 1[doc. 5]). Comme il l'écrivait dans sa lettre au Premier Consul en date du 29 janvier 1803, "Gazola de Vérone, céda, lorsque vous remportiez tant de victoire en Italie, une riche et rare collection de poissons et autres objets pétrifiés, qui forment à présent un des monuments le plus précieux de la Nature. Je n'ai cessé de m'occuper depuis lors, de nouvelles recherches, j'ai fait fouiller dans la montagne de Vestena Nova, et je n'ai rien épargné pour augmenter au moins du double la première collection. Je l'ai offerte à ma Patrie, elle vous en fait hommage par mon organe, et elle est à vous dans ce moment, citoyen Consul, si vous daignez l'accepter» (Fig. 11). Gazola suggérait qu'un professeur du Muséum national d'Histoire naturelle l'accompagnât à Vérone afin "de faire constater le nombre et la rareté des objets par un inventaire» et de veiller à leur emballage et leur bon acheminement vers Paris.

Une des motivations de Gazola était le dépôt et la conservation des spécimens originaux figurés et décrits dans l'Ittiolitologia Veronese dans "un Monument public, consacré aux Sciences" (Annexe 1[doc. 17]). Même si Gazola fit preuve d'une indéniable magnanimité (Gaudant 2011b), il faut aussi 


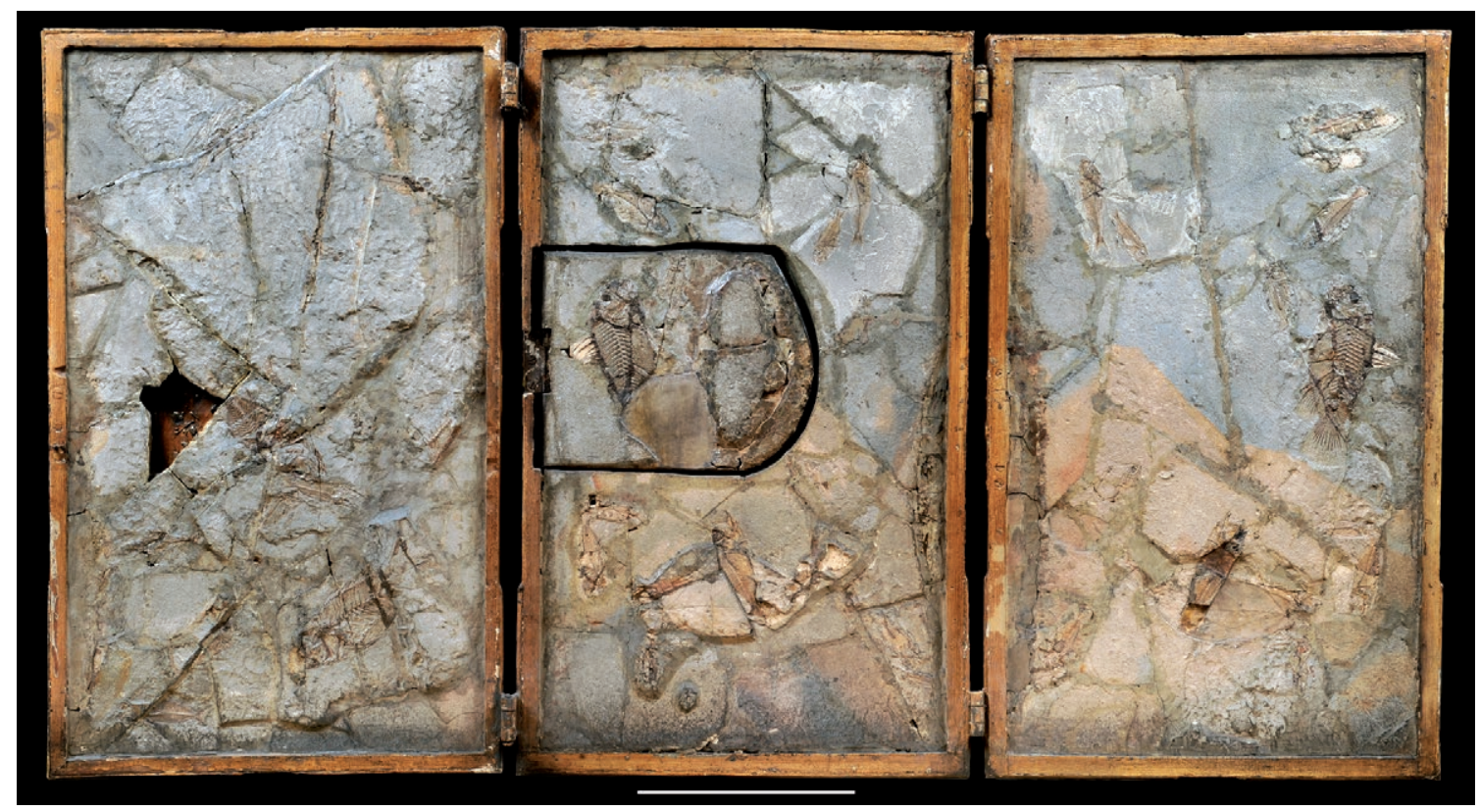

FIG. 13. - Triptyque (MNHN.F.BOL583) offert par Gazola au gouvernement français au nom de la ville de Vérone le 20 floréal an 11 (10 mai 1803 ). Échelle: 20 cm. Crédit: Recolnat (ANR-11-INBS-0004) - Christian Lemzaouda, 2012.

reconnaître que ce cadeau au Premier Consul lui permettait d'espérer une issue favorable à sa demande de complément d'indemnisation pour sa première collection. En ce mois de ventose an 11 (février-mars 1803), le Premier Consul et le gouvernement français devaient donc se prononcer sur deux questions. L'une concernait le complément d'indemnisation réclamé par Gazola pour sa première collection et l'autre portait sur l'acceptation du don de la seconde. En apparence, ces deux pétitions étaient indépendantes, mais dans les faits, elles furent traitées simultanément (Annexe 1[doc. 9]), comme Gazola pouvait s'y attendre.

Bernard-Germain Lacépède (1756-1825), professeur au Muséum, et Jean-Antoine Chaptal, Ministre de l'Intérieur, recommandaient au Premier Consul d'accepter le présent de Gazola (Annexe 1[docs 5, 6]). "Si cette offre doit être acceptée: ce n'est qu'à la sagesse du premier consul qu'il appartient d'en décider. Je prendrai seulement la liberté d'observer que cette collection jointe à celle qui se trouve déjà dans le Museum du Jardin des Plantes formerait un tout bien précieux et peut-être unique dans le monde» écrivait Chaptal en février 1803. Apparemment beaucoup plus soucieux de ne pas priver Vérone d'une telle collection que ne l'avait été le gouvernement français durant la première campagne d'Italie, Chaptal continuait ainsi : «en acceptant cet hommage, Cit[oyen] 1er Consul, vous n'aurez pas à regretter de voir la ville de Vérone privée, sans ressources, de ces objets précieux, puisqu'il lui sera facile de les remplacer par les nouvelles recherches qu'elle peut ordonner de faire dans la montagne de Vestena Nova, mine vraiment inépuisable de ce genre de curiosité» (Annexe 1[doc. 10]). Le gouvernement par un arrêté prit le 11 germinal an 11 (1er avril 1803), accepta officiellement "la collection de poissons pétrifiés, dont la ville de Verone avait chargé Gazola de faire hommage au premier Consul» (Annexe 1[docs 11, 12, 16]). Le ministre de l'Intérieur adressa personnellement ses remerciements à Gazola le 23 germinal an 11 (13 avril 1803): "je vous prie de vouloir bien être auprès de vos concitoyens, l'organe de mes remerciemens particuliers; c'est à leur offre que notre cabinet d'hist: naturelle devra le complément de ce qu'il possédait déjà dans ce genre de curiosité»(Annexe 1[doc. 14]).

Chaptal était également favorable au paiement de la créance de 86500 francs réclamés par Gazola pour sa première collection (Annexe $1[$ docs 7,8$]$ ): "maintenant, si on considère de quel intérêt cette question est pour l'histoire naturelle, ce qu'elle a couté au Cen Gazola pour l'augmenter de toutes celles du même genre qui existaient à Vérone, on ne trouvera pas que la somme demandée soit exorbitante». Le ministre de l'Intérieur avait le soutien de Berthollet, qui était alors sénateur. Chaptal envoya donc une proposition d'ordonnance en ce sens au gouvernement (Annexe 1[doc. 8]). Il y eut manifestement des réticences pour verser une telle somme en une seule fois. Il fut donc décidé d'octroyer à Gazola, par un décret en date du 12 germinal an 11 (2 avril 1803), "une gratification annuelle de 2500 francs" (Annexe 1 [doc. 14] ; Fig. 12). Cette gratification, prise sur les fonds du ministère de l'Intérieur, venait s'ajouter à la rente annuelle d'environ 2300 francs qu'il percevait déjà depuis septembre 1797 sur des terres dans le Mantouan. Le 23 germinal an 11 (13 avril 1803), Gazola fut informé de ces deux décisions, à savoir que le gouvernement français acceptait la seconde collection de poissons pétrifiés, et qu'il lui était par ailleurs accordé 2500 francs par an «en acquit de ce qui restait dî sur le prix» de la première collection cédée en 1797 (Annexe 1[doc. 14]).

La collection qu'il offrait au Premier Consul étant officiellement acceptée, Giovanni Battista Gazola prit contact avec les professeurs administrateurs du Muséum le 20 floréal an 11 (10 mai 1803) pour déjà leur remettre les quelques spécimens 
du Monte Bolca qu'il avait apportés avec lui à Paris et qu'il leur avait présentés en janvier (Annexe 1[doc. 17]). Ce matériel comprenait entre autres le triptyque exposant « 3 morceaux de pierres contenant des poissons \& plantes \& $\mathrm{c}$ fossiles, insérés dans une seule caisse, et qui proviennent de la formation de la Montagne d'où l'on tire ces fossiles» (Fig. 13), quatre décapodes et une plume d'oiseau. Celle-ci avait été achetée vers 1793 par Ignazio Ronconi à des ouvriers indélicats, qui l'avaient extraite de la portion de carrière dont Gazola était propriétaire. Ce dernier s'en était ensuite rendu acquéreur lorsqu'il acheta quelques années plus tard le cabinet de Ronconi. Quelquesunes des pièces offertes par Gazola firent l'objet d'une note de Barthélémy Faujas de Saint-Fond (1804) insérée dans les Annales du Muséum national d'Histoire naturelle.

\section{ACTE 5}

ÉCHEC DE LA MISSION DE LOUIS AUGUSTIN

Guillaume Bosc À Vérone ET SES CONSÉQuenCES

(AVRIL 1803-FÉVRIER 1804)

Jean-Antoine Chaptal fut officiellement chargé d'organiser l'acheminement de la seconde collection Gazola à Paris (Annexe 1[doc. 12]). Il fallait d'abord qu'il nommât un naturaliste capable de s'acquitter de cette mission de confiance. Le nom de Faujas de Saint-Fond circula en premier (Annexe 1 [docs 5, $6,10]$ ) mais c'est finalement Louis Augustin Guillaume Bosc qui fut choisi (Fig. 14). Originaire d'une ancienne famille protestante des Cévennes, Bosc naquit à Paris, le 29 janvier 1759 (Silvestre 1829). Son père était médecin du roi et sa mère était la fille d'un officier d'artillerie. Après des études à Dijon, il rentra dans l'administration des postes. Sa passion pour l'histoire naturelle le conduisit à suivre les cours dispensés au Jardin du Roi. Naturaliste reconnu, il participa à la fondation de la Société linnéenne de Paris, en 1787. Il fut favorable aux idées nouvelles de la Révolution, mais son soutien aux Girondins lui fit craindre d'être persécuté après les journées insurrectionnelles du 31 mai et du 2 juin 1793. Il dut se cacher en forêt de Montmorency jusqu’à la chute de Robespierre en juillet 1794. Son ami Louis-Marie de La Révellière-Lépeaux (1753-1824), membre du directoire exécutif, lui permit de partir pour les États-Unis d'Amérique où il briguait un poste de vice-consul, puis de consul, qu'il ne parvint finalement pas à obtenir. Il profita de ce voyage pour constituer une importante collection d'histoire naturelle, qu'il rapporta en France. À son retour, en 1799, il occupa momentanément différentes fonctions dans l'administration des hospices, des prisons et du Mont-de-Piété. Au début du Consulat, Bosc n'avait cependant plus de poste attitré. La mission en Italie que lui confiait Chaptal arrivait donc à point nommé.

Le 28 floréal an 11 (18 mai 1803), Chaptal envoya à Bosc ses instructions (Annexe 1[doc. 19]). Avant son départ de Paris, Bosc devait d'abord consulter les administrateurs du Muséum national d'Histoire naturelle sur les moyens d'éviter les accidents qu'avait subis la première collection de poissons fossiles durant son transport à Paris. Pendant son voyage, il était chargé de prévenir les professeurs administrateurs du Muséum s'il découvrait, dans les collections qu'il visiterait, des objets inédits et de leur faire part de toutes observations intéressantes concernant la géologie ou les autres branches des sciences naturelles. À son arrivée à Vérone, Bosc devait prendre contact avec les autorités de la ville. Après leur avoir fait connaître son titre et l'ampliation de l'arrêté du 11 germinal, "portant acceptation de la collection des poissons pétrifiés», il devait établir un inventaire de la collection mentionnant le nombre, l'état de conservation et la rareté des objets qui la composaient. Un double de cet inventaire devait être envoyé au ministre de l'Intérieur en personne. Bosc était ensuite mandaté pour contracter tous les marchés nécessaires à l'emballage des objets et leur transport. Enfin, il était chargé de visiter le gisement d'où étaient extraits les "poissons pétrifiés» afin de communiquer aux professeurs du Muséum tous les renseignements "propres à avancer la science de la géologie». Pour l'ensemble de cette mission, Chaptal lui avait alloué un budget de 4000 francs.

La mission de Bosc à Vérone n'avait pas commencé que les bases d'un malentendu entre Gazola et le ministre de l'Intérieur se mettaient en place. Dans sa première lettre, en date du 29 janvier 1803, dans laquelle il offrait à Napoléon Bonaparte une nouvelle collection de poissons fossiles, Gazola laissait entendre qu'elle avait été remise à la ville de Vérone et qu'elle était deux fois plus importante que la première (Annexe 1[doc. 5]). C'est d'ailleurs sur ces éléments que le gouvernement français prit la décision d'accepter ce présent. Dans sa lettre du 20 floréal an 11 (10 mai 1803) aux professeurs administrateurs du Muséum, Gazola semblait être beaucoup plus restrictif sur le choix des spécimens qu'il comptait livrer : "je n'ai offert moi-même à la ville de Véronne [sic] pour être donné au Gouvernement Français, que les poissons nécessaires, pour completter [sic] ceux qui manquent à la grande collection du Muséum» (Annexe 1[doc. 17]). Il demandait, en conséquence, à ce que Bosc s'entendît avec l'administration du Muséum pour établir un inventaire précis de tous les poissons que le Muséum possédait pour l'aider dans le choix des spécimens à envoyer à Paris. Giovanni Battista Gazola recommandait qu' « il faut éviter d'envoyer des doubles dont le Muséum n'a déjà qu'un très-grand nombre». Au lieu d'imposer sa manière de voir, qui après tout était légitime, Gazola commit l'erreur de charger les administrateurs du Muséum de demander au ministre de l'Intérieur de trancher la question de savoir si tout ou partie de sa seconde collection devait être emportée. René Desfontaines (1750-1833), qui était alors directeur du Muséum, et Alexandre Brongniart (1770-1847), qui en était le secrétaire, soumirent le problème à Chaptal dans une lettre en date du 26 floréal (16 mai 1803) (Annexe 1 [doc. 18]).

De son côté, Bosc se rendit au Muséum pour se renseigner, conformément aux ordres du ministre, sur la manière dont il devait emballer et faire transporter la collection de poissons fossiles (Annexe 1[doc. 20]). Il fut très surpris d'apprendre que cette collection n'appartenait en réalité pas vraiment à la ville de Vérone, mais était encore en possession de Gazola, qui l'avait offerte au Premier Consul au nom de la ville. Il apprit de surcroît que le collectionneur italien était encore à Paris et qu'il venait de faire envoyer à Chaptal une lettre «dans laquelle il semblait restreindre l'etendue [sic] du don acceptépar le Gouvernement et y mettre des conditions». Sur ces entrefaites, 
Bosc rencontra Gazola, qui lui confirma qu'il avait l'intention de ne donner au gouvernement, pour le Muséum d'histoire naturelle, que les espèces qui manquaient. Bosc croyait quant à lui devoir prendre tous les spécimens bien conservés qui méritaient, à ses yeux, les frais du transport, même s'ils appartenaient à des espèces identiques à celles déjà présentes au Muséum. De surcroît, Bosc voulait partir pour Vérone dès le mois de juin, afin de profiter de la saison favorable aux observations zoologiques et botaniques. Gazola dont la mission à Paris n'était pas encore achevée, voulait au contraire que le naturaliste français remît son voyage à l'hiver suivant pour lui permettre de l'accompagner et choisir avec lui les poissons fossiles qui seraient donnés au Muséum. Bosc prit donc la plume le 5 prairial (25 mai 1803) pour réclamer au ministre de trancher la question : "je vous prie, en conséquence, Citoyen Ministre, de vouloir bien vous faire remettre sous les yeux les pieces fondamentales de cette affaire, vous entendre avec M. de Gazola, et me faire part de votre détermination ultérieure".

Chaptal ordonna à l'un de ces collaborateurs, Jean-Pierre Barbier-Neuville (1754-1822), de rédiger un rapport pour l'aider dans sa décision. Barbier-Neuville naquit le 29 août 1754 à Vitry-le-François, dans le département de la Marne. Il rentra au ministère de l'Intérieur en qualité de secrétaire général le $1^{\mathrm{er}}$ messidor an 7 (19 juin 1799) (base Léonore, dossier LH/110/92). Il fut nommé chef de la 3e division de ce ministère, le 1 er frimaire an 9 (22 novembre 1800$)$, poste qu'il occupa jusqu'au 1er juin 1814, avant d'être nommé directeur des correspondances. Il prit sa retraite à la fin de l'année 1815 et décéda le 5 janvier 1822. L'administration du Muséum national d'Histoire naturelle dépendait alors de la $3 \mathrm{e}$ division du ministère de l'Intérieur qui gérait toutes les questions afférentes aux hospices, aux bâtiments civils et aux établissements des sciences, des lettres et des arts. Barbier-Neuville rendit son rapport au ministre le 12 prairial (1er juin 1803). Il fit remarquer que même si des doubles étaient rapportés de Vérone, certains pourraient se trouver dans un meilleur état de conservation que ceux qui étaient conservés au Muséum. Il serait également possible de constituer des collections qui seraient accordées à d'autres établissements publics. Il continuait son rapport en notant que " tout ce qu'on pourrait en écarter, et même avec quelque ménagement, ce serait ces morceaux composés de parties brisées de différents poissons (à supposer qu'il en existe de pareils dans cette collection) peut-être y aurait-il de l'avantage à les emporter tous, ne fut-ce que pour prouver que c'est ce genre de fourberie qu'il faut attribuer la persuasion où l'on est que les montagnes du Véronais présentent un plus grand nombre de poissons inconnus aux nomenclateurs qu'il ne s'en trouve réellement» (Annexe 1[doc. 21]). Barbier-Neuville faisait enfin remarquer que Gazola n'avait mis aucune restriction dans l'offre initiale qu'il avait faite au Premier Consul. Il proposait en conséquence au ministre que la collection de poissons pétrifiés, offerte au gouvernement français par la ville de Vérone, fût transportée à Paris dans sa totalité. Chaptal suivit les recommandations de son conseiller et une semaine plus tard, le 19 prairial (8 juin 1803), il envoya une lettre à Bosc lui ordonnant de partir sur le champ à Vérone pour rapporter à Paris la totalité de la seconde collection de poissons

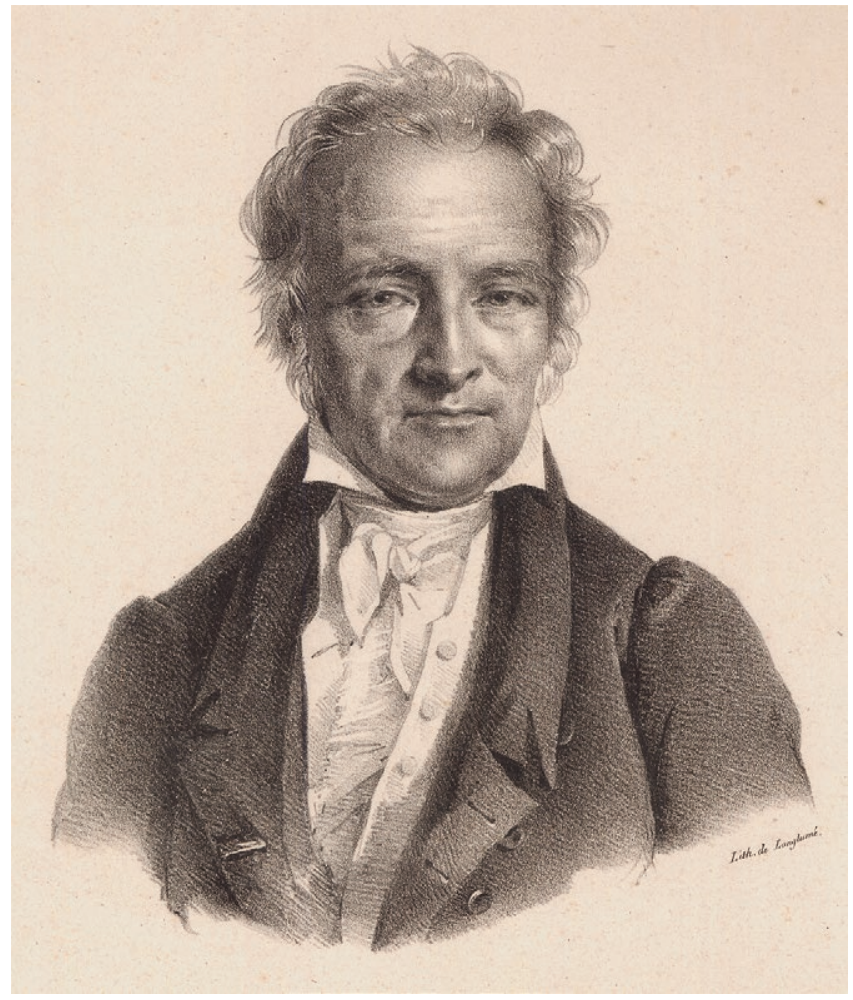

FIG. 14. - Louis Augustin Guillaume Bosc, ou Bosc d'Antic (29 janvier 1759, Paris - 10 juillet 1828, Paris), portrait lithographié par Pierre Langlumé (17901830), (c) Muséum national d'Histoire naturelle, Paris, cote Po. 1316.

fossiles formée par Gazola (Annexe 1[doc. 22]). Les professeurs administrateurs du Muséum furent également prévenus de cette décision et étaient invités à en informer Gazola. On imagine quelle fut sa surprise quand il apprit que Bosc partait à Vérone sans lui pour rapporter l'ensemble de sa collection ! Certes, Chaptal tranchait une question que Gazola lui avait posée, mais cette décision unilatérale prise sans concertation avec le principal intéressé portait les germes d'une crise qui allait devenir une véritable affaire d'état.

Bosc arriva à Vérone vers la mi-juillet (Annexe 1[doc. 23]). Comme prévu, il se présenta aux autorités de la ville pour leur faire part de l'objet de sa mission. La collection de poissons fossiles qu'il venait chercher se trouvait dans le palais Gazola. Les administrateurs arguèrent qu'ils ne pouvaient rien faire en l'absence de son propriétaire qui rappelons-le était encore à Paris. Bosc accepta de leur laisser le temps nécessaire pour obtenir des instructions de Gazola. Bosc en profita pour explorer la montagne du Vicentin et les bords de la mer Adriatique (Annexe 1 [doc. 25]). Au cours de cette tournée, il visita le Monte Bolca et la carrière des poissons fossiles pour faire les observations géologiques que lui avait demandées Jean-Antoine Chaptal. Il visita également plusieurs collections intéressantes. Un mois environ s'était écoulé quand Bosc retourna à Vérone, persuadé que la collection de poissons fossiles allait être mise à sa disposition. Il n'en était rien. Le 16 août 1803, les administrateurs de la ville firent lire à Bosc une lettre de Gazola, en date du 31 juillet, dans laquelle il "persistait» à mettre des «restrictions» sur le don qu'il 
TABLEAU 1. - Inventaire d'une partie de la collection Gazola réalisé par Louis Augustin Guillaune Bosc, à Vérone, en août 1803.

\begin{tabular}{|c|c|c|c|}
\hline & $\begin{array}{l}\text { Nombre } \\
\text { de } \\
\text { plaques } \\
\text { doubles }\end{array}$ & $\begin{array}{l}\text { Nombre } \\
\text { de } \\
\text { plaques } \\
\text { simples }\end{array}$ & $\begin{array}{l}\text { Nombre } \\
\text { total de } \\
\text { pièces }\end{array}$ \\
\hline Poissons du Monte Bolca & 112 & 39 & 263 \\
\hline $\begin{array}{l}\text { Restes de végétaux du } \\
\text { Monte Bolca }\end{array}$ & 11 & 4 & 26 \\
\hline Crustacés du Monte Bolca & - & 2 & 2 \\
\hline $\begin{array}{l}\text { Autres fossiles ne provenant } \\
\text { pas du Monte Bolca }\end{array}$ & - & - & 43 \\
\hline Total & 123 & 45 & 334 \\
\hline
\end{tabular}

avait fait au nom de la ville au Premier Consul, et ce, malgré la décision du ministre de l'Intérieur (Annexe 1[doc. 23]). Bosc était visiblement furieux d'avoir ainsi attendu en vain pendant un mois. Il transmit à la municipalité, le 17 août, le message suivant : "je dois vous repetter [sic] aujourd'hui par ecrit [sic] ce que je vous ai dit hier de vive voix, Citoyens administrateurs, c'est qu'il ne m'est plus permis de discuter cette matière avec vous. La lettre du Ministre de l'Intérieur, dont j'ai l'honneur de vous envoyer copie, me faisant une loi de reclamer auprès de vous la collection entiere, ou de ne recevoir de vous que la collection complette existant chez $M$. de Gazola a ce que vous m'avez annoncée, mais devant s'y trouver facilement». En d'autres termes, Bosc intimait l'ordre aux autorités de Vérone de pénétrer dans le palais Gazola afin qu’il pût emporter toute la collection qui s'y trouvait. Les administrateurs de la ville envoyèrent dès le lendemain une missive à Gazola pour le prévenir (Annexe 1[doc. 24]). Ils avaient bien essayé d'argumenter que le don fait au Premier Consul avait pour but de compléter la première collection qui se trouvait au Muséum et que l'envoi d'un grand nombre de doubles de la même espèce était superflu. Mais Bosc leur rétorqua que ces doubles pourraient être offerts aux frères du Premier Consul et seraient utiles à d'autres établissements publics sur le territoire français. Il finit par les menacer en leur disant qu'on ne plaisantait pas avec une décision du gouvernement et qu'il ne repartirait qu'avec la totalité de la collection ou rien du tout. Les représentants de la ville craignant ainsi l'incident diplomatique finirent, prudemment, par obtempérer. La famille de Gazola et notamment ses frères furent priés de donner accès à la fameuse collection et un commissaire, en la personne de Giuseppe Rotari, fut nommé pour assister Bosc. Marescalchi, le ministre des Affaires extérieures de la République italienne, reprocha plus tard aux officiers de la ville de Vérone, de s'être "laissé un peu trop facilement intimider» (Zaghi 1961: 103).

Quelques jours plus tard, le 3 fructidor (21 août 1803), Giovanni Battista Gazola, qui n'avait pas encore reçu la lettre de ses concitoyens et qui ne se doutait donc pas de ce qui se tramait chez lui, obtenait enfin une audience auprès du Premier Consul. Les requêtes qu'il soumit, relatives au rôle prépondérant qu'entendait jouer la ville de Vérone au sein de la République italienne, trouvèrent une issue favorable. L'hommage qu'il faisait d'une nouvelle collection de poissons du Monte Bolca ne pouvait que lui apporter les grâces du chef d'État français. Les journaux parisiens et milanais de l'époque se firent l'écho de l'évènement (Journal de Paris 1803: 2144 ; Journal des débats 1803: 2 ; Il Corriere Milanese 1803: 565) : «à l'audience qui eut lieu dimanche dernier, 3 fructidor, le ministre des relations extérieures de la république italienne [Francesco Marescalchi], présenta au premier consul les C.ns [Citoyens] Gazola \& Pinali, députés de la ville de Vérone. Ils étoient venus, il y a quelque temps, à Paris, pour solliciter quelques priviléges [sic] particuliers, que la situation de leur ville rendoit nécessaires. Le premier consul a favorablement accueilli leur demande. C'est au C.en Gazola qu'appartenoit la précieuse collection de poissons pétrifiés que l'on voit au muséum d'Histoire naturelle. Cette collection doit être incessamment enrichie d'un grand nombre de pièces nouvelles recueillies par le même naturaliste, \& que la ville de Vérone, à qui il en avoit fait présent, a offertes au premier consul. Le C.en Bosc est nommé pour aller les recevoir \& veiller à leur transport». Personne à Paris ni à Milan ne se doutait alors que la situation était en train de dégénérer.

Pendant ce temps, Bosc travaillait inlassablement au catalogue de la collection complète de Gazola et à son emballage. La municipalité paya les caisses et le matériel nécessaire au conditionnement des pièces, si bien que Bosc n'eut à débourser qu'une petite somme pour récompenser les ouvriers et les domestiques qui l'aidaient (Annexe 1[doc. 25]). Son travail terminé, Bosc écrivit au ministre de l'Intérieur le 29 août 1803 pour lui communiquer l'inventaire de la collection sur lequel il avait "grossierement figuré chaque espece de poisson afin qu'on puisse se former une idée de sa forme». Ce document précieux, conservé aux Archives nationales, est reproduit dans son intégralité dans l'Annexe 2 et permet de se faire une idée sur l'état d'une grande partie de la collection Gazola en 1803. Une partie, car en effet, comme nous le verrons dans le chapitre suivant, les frères de Gazola avaient eu le temps de dissimuler un certain nombre de pièces précieuses. La collection répertoriée par Bosc comprenait 263 poissons en comptant les doubles plaques, 26 restes de végétaux et deux crustacés, soit un total de 291 pièces du Monte Bolca (Tableau 1). À cellesci s'ajoutaient 43 fossiles provenant d'autres localités. Bosc écrivait que plusieurs spécimens «sont d'une grande importance et ne se trouvent pas dans la collection du Museum" mais que leur nombre n'était pas aussi considérable que Gazola l'avait annoncé (Annexe 1[doc. 25]). À la demande de Chaptal, il avait également reçu de Pierre Thouvenel (1745-1815), médecin français originaire des Vosges, 300 exemplaires de son ouvrage sur le climat de l'Italie (Thouvenel 1797-1798). La collection Gazola et ces livres occupaient 33 caisses, pesant au total entre deux et trois tonnes. Bosc paya 600 francs pour les frais de transport de Vérone à Milan d'où il devait recontacter Chaptal pour l'informer de la suite des opérations.

Enfin alerté de ce qui venait de se passer à Vérone, Giovanni Battista Gazola prit la plume pour implorer la protection de Napoléon Bonaparte. Il se garda bien de mettre en cause le ministre de l'Intérieur préférant incriminer Bosc qui ne faisait pourtant qu'obéir scrupuleusement aux ordres de Chaptal (Annexe 1[doc. 26]). La lettre de Gazola commence ainsi : "Vous daignâtes il y a quelques mois accepter une offre que la 
Commune de Vérone avait pris la liberté de vous faire par mon organe. Heureux d'avoir obtenu de vous cet acte de bonté pour moi et mes Concitoyens, jétais loin de prévoir qu'il deviendrait l'occasion d'une entreprise contre laquelle je serais forcé d'implorer votre justice et votre protection». Le collectionneur de Vérone reprochait à Bosc de s'être hâté de partir en Italie au lieu de l'attendre et d'avoir menacé et effrayé les officiers municipaux pour que lui fût livré indistinctement tout ce qui était chez lui. Gazola poursuivait sa lettre en ces termes: "Ainsi donc, Citoyen Premier Consul, dans ce moment même l'on s'est emparé de tout le fruit de mes longs et pénibles travaux. Ainsi, en me privant de tout ce que j'ai recueilli, on a renversé tous les projets que je formais, soit pour le progrès de la science, soit pour l'utilité de nos établissemens publics. Ainsi, en un mot, l'on m'a porté un des coups les plus sensibles dont je pusse être frappé. Mais une espérance me reste et assurément elle ne sera point trompée. C'est que votre justice, votre générosité ne souffriront pas que l'on me dépouille de cette manière. C'est que vous voudrez bien ordonner que tout ce qu'on m'a pris me soit rendu et que si même les caisses sont déjà parties de Vérone, elles soient arrêtées partout où elles se trouveront et reportées chez moi pour que j'en sépare tout ce qui n'a point du être livré». Gazola réitéra sa volonté d'offrir les spécimens qui manquaient au Muséum mais souhaitait bien entendu conserver tout le reste, soit pour son propre usage, soit dans le but d'enrichir les cabinets de Pavie et de Bologne.

Également prévenu par Gazola, le ministre des Affaires extérieures de la République italienne, Marescalchi, en parla immédiatement au Premier Consul. Napoléon Bonaparte fut "fort mécontent» et donna "l'ordre de prendre les mesures nécessaires pour tout réparer" (Zaghi 1961: 103, lettre 1318). En conséquence, Marescalchi écrivit de Paris, le 29 août 1803, une lettre au Vice-Président de la République italienne, Francesco Melzi d'Eril (1753-1816), qui se trouvait à Milan, pour lui demander d'empêcher Bosc de rentrer en France avec les caisses: "si, comme il y a lieu de le craindre, la collection est déjà en route, vous voudrez bien donner vos ordres pour qu'on l'arrête, malgré toute opposition que pourrait faire le citoyen Bosc. En un mot, je vous prie d'agir, ainsi que les circonstances pourront l'exiger, pour empêcher que la singulière entreprise de ce citoyen soit consommée et que le citoyen Gazola soit dépouillé du fruit de ses travaux contre le désir du Premier Consul» (Zaghi 1961: 104). Marescalchi avait joint à sa lettre une missive destinée aux officiers municipaux de la ville de Vérone au cas où Bosc y serait encore. Le ton de Marescalchi était des plus autoritaires et comme il l'expliquait à Melzi d'Eril: "j'ai cru devoir le faire ainsi non pour les réprimander, car je n'en ai point le dessein, ni ne prétends m'en arroger le droit, mais pour leur donner un titre dont ils puissent s'appuyer contre toute opposition de la part du citoyen Bosc, qui sans doute cherchera à se prévaloir de sa commission et des ordres du ministre Chaptal. Au fond, néanmoins, il n'est pas mal, ce me semble, que les agents et les employés de notre République apprennent qu'ils ne doivent respect et obéissance qu'aux ordres de leur propre Gouvernement" (Zaghi 1961: 104). Mais quand Melzi d'Eril transféra les ordres de Marescalchi aux administrateurs de Vérone, il était trop tard: Bosc était déjà parti pour Milan. Ayant conclu les affaires qui le retenaient jusqu'alors à Paris, Gazola estima qu'il était opportun de rentrer en Italie de toute urgence pour tenter de récupérer son bien.

Lorsque Bosc arriva à Milan, son convoi fut saisi par la douane. Melzi d'Eril s'empressa d'écrire à Marescalchi, le 9 septembre 1803, pour le prévenir (Zaghi 1961: 133, lettre 1356). Bosc sollicita une entrevue avec le Vice-Président pour contester cette décision mais Melzi d'Eril rétorqua qu'il ne faisait qu'appliquer un ordre du Premier Consul. Il exhortait Bosc d'attendre l'arrivée imminente de Gazola, qui avait seul le pouvoir de lever la saisie et de séparer les pièces qu'il acceptait de donner de celles qu'il désirait conserver. Gazola rencontra enfin Bosc à Milan. On imagine que la rencontre entre les deux hommes fut des plus glaciales. Il était certainement impossible dans ces conditions de choisir les spécimens qui manquaient au Muséum national d'histoire naturelle et Gazola préféra remettre à plus tard cette sélection. Le 14 septembre, Bosc écrivit à Chaptal pour l'informer qu'il retournait en France le lendemain par la route la plus directe n'emportant avec lui que les caisses qui contenaient les ouvrages de Thouvenel (Annexe 1[doc. 27]). De son côté, Gazola rapatria à Vérone sa collection. Il laissa cependant en dépôt à Milan les deux grandes caisses contenant les spécimens les plus volumineux. L'un d'eux avait en effet souffert pendant son transport et s'était brisé en deux (il s'agit vraisemblablement du spécimen MNHN.F.BOL544a, BOL544b). Comme l'écrivit plus tard Faujas de Saint-Fond, "ce n'étoit certainement pas la faute de Bosc, mais une aussi grande caisse étant difficile à manier, et les roulliers étant très peu soigneux, ils la déchargèrent des charettes sans ménagement» (Annexe 1[doc. 47]). Dans son départ précipité de Milan, Bosc oublia même une caisse qui contenait des insectes et des minéraux qu'il avait lui-même collectés durant son voyage en Italie.

Cette mission fut donc un terrible échec pour le naturaliste français d'autant qu'il s'était "considérablement déplu de perdre dans les auberges un temps" qu'il aurait "eu mieux employé autre part» comme il l'écrivit à Giuseppe Angelo Saluzzo di Menusiglio (ou Joseph-Ange, comte de Saluces de Menusiglio) (1734-1810), président de l'Académie des Sciences de Turin (Annexe 1[doc. 28]). S'étant montré loyal envers Jean-Antoine Chaptal, Bosc fut nommé à son retour en France, au début du mois d'octobre 1803, inspecteur des pépinières de Versailles (Annexe 1[doc. 29]). De son côté Gazola fut accueilli à Vérone comme un héros, ayant obtenu pour la municipalité les faveurs et la protection du Premier Consul et Président de la République italienne (Nicolis 1907: 48; Blot 1969: 11 ; Gaudant 2011b: 646). Le citoyen Dariff, membre du Conseil municipal prononça même, le 7 novembre 1803, un vibrant hommage à Gazola le remerciant du don qu'il avait fait de sa collection de poissons fossiles qui, rappellons-le, restait finalement chez lui!

Quant à Chaptal, il avait essuyé un tel camouflet dans cette affaire, qu'il fit prendre des dispositions pour suspendre le 1er brumaire an 12 (24 octobre 1803), le versement de la gratification annuelle de 2500 francs que Gazola avait obtenu pour le complément de paiement de la première collection cédée en 1797 (Annexe 1[docs 34, 39, 57]). Ferdinando Marescalchi tenta de plaider à nouveau en faveur de Gazola 


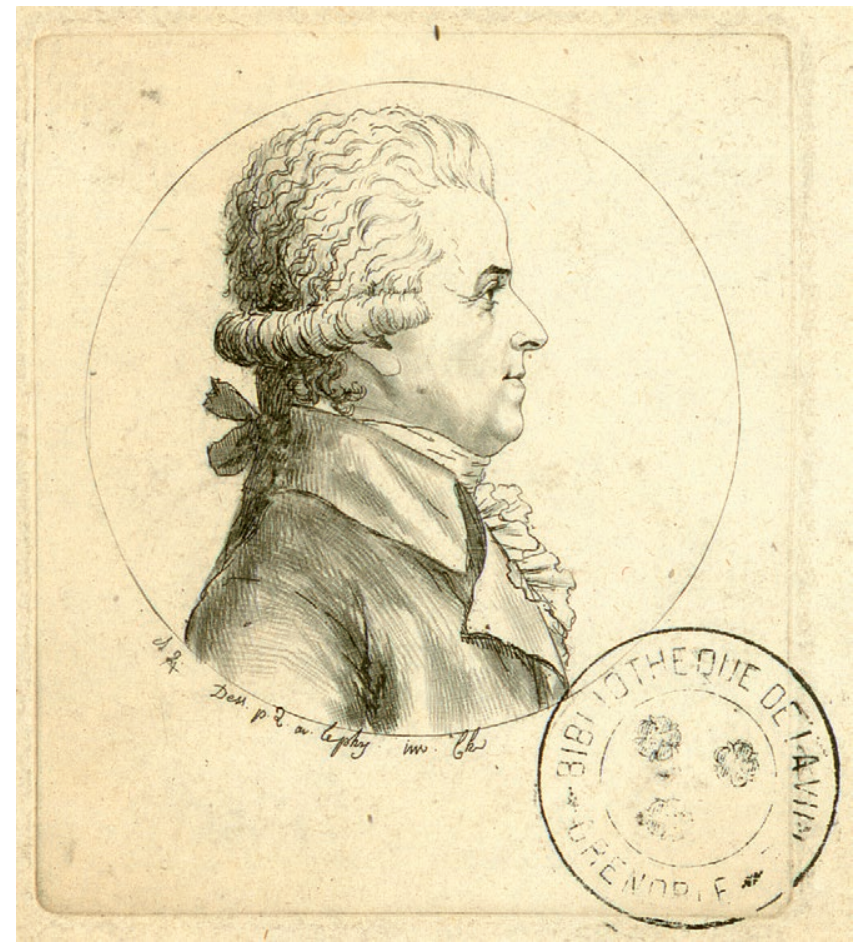

FIG. 15. - Barthélémy Faujas de Saint-Fond (17 mai 1741, Montélimar - 18 juille 1819, Loriol-sur-Drôme) par Edme Quenedey des Riceys (1756-1830) réalisé avec le physionotrace de Gilles-Louis Chrétien (1754-1811) (Bertrand 2008: pl. 5), (C) Bibliothèque municipale de Grenoble, Pd.1 Faujas (Barthélémy) (2).

et écrivit, le 2 janvier 1804, une longue lettre à Chaptal rappelant toute l'affaire (Annexe 1[doc. 33]). Là encore, Bosc était présenté comme l'unique responsable de cette "désagréable affaire" alors qu'il n'était que le bras droit du ministre de l'Intérieur. Cette tentative de Marescalchi pour faire obtenir la pension à laquelle Gazola avait droit fut vaine. Le 9 ventose an 12 (29 février 1804), Jean-Antoine Chaptal écrivit à François Mami, le fondé de pouvoir de Gazola à Paris : «vous minvitez, Citn à faire acquitter les arrérages de la pension qui lui a été accordée pour terminer le payement de la collection qu'il a vendue en l'an 5 au Gouvernement. Je vous préviens qu'il ne sera donné suite à une demande que lorsque le Citn Gazola aura rempli l'engagement qu'il a contracté, et que la collection de poissons fossiles, offerte par la ville de Vérone, sera effectuée» (Annexe 1[doc. 36]).

Chaptal, qui s'était d'abord montré libéral et compréhensif avec Gazola dans sa demande d'indemnisation, fit ensuite preuve d'une indéniable rigidité et d'un excès d'autoritarisme; une ambivalence qui fait écho à l'une de ses récentes biographies intitulée "Chaptal, un libéral autoritaire» (Gueniffey 2011). Il était après tout bien légitime que Gazola fixât luimême les limites du cadeau qu'il souhaitait offrir au Premier Consul. Dans cette affaire, il est indéniable que Chaptal fut mal conseillé par Barbier-Neuville, qui avait recommandé au ministre d'ordonner à Bosc de rapporter de Vérone l'ensemble du cabinet Gazola. Il convient aussi de souligner que le collectionneur véronais fut quelque peu maladroit avec sa lettre aux administrateurs du Muséum dans laquelle il demandait au ministre de trancher la question de savoir si tout ou partie de sa collection devait être rapportée à Paris (Annexe 1[doc. 18]). Enfin, Bosc tient aussi sa part de responsabilité dans l'échec de sa mission, ayant insisté pour partir sans attendre d'être accompagné par Gazola. Dans une lettre au Vice-Président de l'Italie en date du 17 septembre 1803, Marescalchi se réjouissait que le cabinet d'histoire naturelle "del povero Gazzola" [de ce pauve Gazola] avait été sauvé juste à temps. Il reprochait également à Bosc et Chaptal d'avoir abusé de leur autorité à l'insu du Premier Consul (Zaghi 1961: 142, lettre 1370).

ACTE 6

Mission de Faujas De Saint-Fond à Vérone et arrivée À PARIS de la COLlection Gazola 2 (1804-1806)

Giovanni Battista Gazola promit d'envoyer au printemps de l'année 1804 la collection de poissons qu'il destinait au Muséum (Annexe 1[doc. 35]). Mais les choses n'étaient pas aussi simples car il fallait pour cela qu'un nouveau commissaire français fût désigné pour organiser son transport. En France, la situation politique évoluait rapidement. Le 18 mai 1804, une nouvelle constitution était adoptée et Napoléon Bonaparte était proclamé Empereur des Français. Chaptal quittait, quant à lui, ses fonctions de ministre le 7 août 1804 , pour devenir sénateur. En attendant que le nouveau ministre de l'Intérieur, Jean-Baptiste de Nompère de Champagny (17561834), rentrât de Vienne où il était ambassadeur depuis 1801, l'intérim du ministère fut assuré par le ministre des Cultes, Jean-Étienne-Marie Portalis. Né le 1er avril 1746 au Beausset dans le Var et mort à Paris le 25 août 1807, Portalis était avocat sous l'Ancien Régime et jouissait d'une belle situation quand la Révolution éclata. Condamné comme royaliste, il dut son retour en grâce à Napoléon Bonaparte qui estimait ses talents. Il est notamment connu pour avoir été l'un des rédacteurs du Code civil. En tant que ministre de l'Intérieur par intérim, ce fut donc au tour de Portalis de prendre en main le «dossier Gazola». En accord avec les termes imposés par ce dernier, il fallait trouver un commissaire qui eût une bonne connaissance de la collection présente au Muséum pour ne choisir que les poissons qui manquaient. C'est naturellement Barthélémy Faujas de Saint-Fond, responsable de la collection de géologie du Muséum, qui fut nommé. La décision fut entérinée par un arrêté, le 28 vendémiaire an 13 (20 octobre 1804). Portalis voulait à tout prix éviter de nouveaux incidents diplomatiques et écrivait à Faujas: "j’espère de votre zêle et de votre esprit conciliateur, que vous pourrez obtenir la collection des poissons fossiles que la ville de Veronne avait offerte au Gouvernement, mais que nous ne possédons point encore. Quand vous aurez fixé l'époque de votre départ, je vous remettrai, des notes sur cette affaire et vous indiquerai la marche à suivre pour lever les obstacles qui s'opposent à l'expédition de ces poissons en France» (Annexe 1[doc. 37]).

Faujas de Saint-Fond naquit le 17 mai 1741 à Montélimar (Fig. 15). Son père était greffier en l'élection de cette ville et c'est donc naturellement que le jeune Faujas s'orienta vers la magistrature (Rochas 1856: 375-378; Brignon 2017). Après ses études de droit, il travailla pour un procureur de Grenoble et exerça sa profession d'avocat au Parlement. En 
1765, il fut nommé vice-sénéchal à Montélimar. Malgré cette position honorable, ses centres d'intérêt se tournaient ailleurs et en particulier vers les sciences naturelles et la géologie. Ses Recherches sur les volcans éteints du Vivarais et du Velay, publiées en 1778, lui permirent d'acquérir une notoriété auprès du monde savant de l'époque. Dès 1776, il entretenait une correspondance scientifique avec Buffon, qui était alors l'administrateur du Jardin du Roi. Sur la recommandation du grand naturaliste, Faujas de Saint-Fond fut nommé en 1779 adjoint aux travaux de cet établissement (Freycinet 1820). Quelques années plus tard, en mai 1785, il reçut le titre de commissaire du roi pour les mines. À la fondation du Muséum national d'Histoire naturelle, créé en 1793 à partir du Cabinet et du Jardin du Roi, Faujas de Saint-Fond obtint la chaire de géologie, qu'il occupa jusqu'en 1818. En qualité de Commissaire chargé de rechercher des objets de science et d'art, Faujas accompagna l'armée révolutionnaire en Hollande en 1794 et 1795 (Lacour 2009). Il participa notamment au transfert en France du "grand animal fossile des carrières de Maestricht», qui servit d'holotype au premier Mosasauridae nommé de l'histoire, Mosasaurus hoffmanni Mantell, 1829 (Bardet \& Jagt 1996; Brignon 2015).

L'arrêté de Portalis ordonnait que Faujas fît son voyage en Italie en l'an 13. Il sécoula environ huit mois pour que Faujas quittât enfin Paris, en juin 1805 (Annexe 1[doc. 49]). Il resta tout l'été dans le Sud de la France et en Ligurie. Il fit divers observations sur les poissons fossiles des carrières à plâtres d'Aix-en-Provence, sur la faune ichtyologique du golfe de Gênes et sur les "brèches coquillières et osseuses» de la région de Nice (Faujas de Saint-Fond 1806a, b; 1807). Le 29 septembre, il quitta Nice et longea la côte. Tout en consignant ses observations géologiques, il traversa Monaco, Roquebrune, le Cap Martin, Menton, Vintimille, Bordighera, San Remo, Oneille, Alassio, Albenga, Savona, Arenzano pour arriver à Gênes (Faujas de Saint-Fond 1808). Son journal de voyage manuscrit conservé aux Archives départementales de l'Isère permet de suivre la suite de son voyage jusqu’à Vérone (Bertrand 2008: 404) (Fig. 16). Le 12 octobre 1805, il quitta Gênes pour rejoindre Campomarone, Voghera, Stradella et arriver à Plaisance le 14 octobre. Il y visita le cabinet d'histoire naturelle de Cortesi et repartit le 16 octobre pour Crémone. Il arriva le 18 à Mantoue, puis le 19 octobre à Villafranca où il apprit que, la veille, avait éclaté la bataille de Vérone. Faujas de Saint-Fond arriva le 20 octobre 1805 à Vérone pour venir chercher la collection de poissons fossiles, alors que la région venait tout juste de sombrer dans un conflit entre les armées française et autrichienne.

La proclamation du Royaume d'Italie et l'annexion de la République ligurienne à l'Empire français avait déterminé l'Autriche à rejoindre, en juin 1805, la Troisième Coalition qui, sous l'égide des Britanniques, s'opposait à Napoléon 1er. Alors que le gros de l'armée française se déployait rapidement dans le sud de l'Allemagne, le général André Masséna (1758-1817) avait pour ordre de maintenir le plus longtemps possible en Italie les troupes autrichiennes dirigées par l'archiduc Charles (1771-1847), frère de l'empereur d'Autriche, François Ier (1768-1835). Au petit matin du 18 octobre 1805, Masséna fit attaquer le pont de Castelvecchio qui enjambe l'Adige et qui reliait Vérone à Véronette occupée par les Autrichiens. Un groupe de quelques hommes parvinrent à franchir le pont et percer la ligne ennemie. Même si Masséna ne parvint pas à conquérir Véronette, les pertes infligées aux Autrichiens furent importantes. Le calme revint le soir même, mais les deux camps se préparaient à de nouveaux affrontements. Dans son journal de voyage, Faujas de Saint-Fond décrit son arrivée à Vérone en ces termes:

20 [octobre 1805] parti de Villafranca, à huit heures du matin, la route étoit entierement embarassée de troupes, de canon, de chariots d'approvisionnement. Nous sommes arrivés malgré cette bagarre à midi à Vérone, où j'étais attendu par M. de Gazola, que j'avois prevenu la veille par une lettre dont un officier de dragon sétoit chargé à Mantoue; il a eu la bonté de me ceder son appartemant et son propre lit, tant il avoit d'officier logé chez lui; $M$. de Gazola est membre de l'administration municipale, qui ayant une armée considérable à nourrir, a de l'ouvrage par-dessus la tête. J'ai diné chez Mr de Gazola avec deux de ses frères, un chef de genie, et un colonnel du ... régiment [sic], qui est de Bayonne, et qui après dinné à bien voulu me conduire dans une maison à la tête du premier pont de la ville sur l'Adige du côté de Veronne française, qui n'est séparé de Vérone autrichienne que par la rivière, un peu moins large que la Seine à Paris, mais beaucoup plus rapide. Ce pont n'a point été attaqué. La ligne de démarcation est au milieu. Les autrichiens qui ont la tête de pont de leur côté, se sont fortement barricadé par des palissades; et ont deux sentinelles en avant, plus une pièce de canon dirigée sur le pont dans la première rue qui est en face; notre côté est également fortement barricadé; il y a en contre des chevaux de frise, et l'on plaçoit deux grosses chaines, pour arretter la cavalerie en cas de surprise, et deux sentinelles en avant très voisins des deux sentinelles autrichiennes. Les uns et les autres très attentifs, immobiles et dans le plus grand silence; les maisons voisines évacuées et garnies de soldats et une pièce de canon masquée à l'entrée du pont. Nous sommes allé [sic] voir ensuite le second pont qui est beaucoup plus bas, où les mêmes précautions respectives ont été prises. Il était trop tard pour aller visiter le troisième pont où l'attaque sétait faite, jirai le voir demain. C'est le pont de Castelvechio [sic]. [...] On s'attend bientôt à une attaque que forme les français qui vont au combat avec un courage veritablement heroïque (ADI, J 546).

Malgré le conflit qui secouait Vérone, Faujas de Saint-Fond n'hésita pas à visiter la ville. Il se rendit notamment, le 21 octobre, aux arènes de Vérone dans lesquelles on pénétrait par la boutique d'un marchand d'estampes et d'histoire naturelle, un certain Gaetano Somma Campagna, homme affable, qui lui servit de guide. Faujas visita son cabinet d'histoire naturelle "qui renferme beaucoup de pétrifications des environs de Vérone, mais mal conservées en général». Il y vit cependant "quelques poissons et empreintes de plantes de Vestenanova". 
Faujas acheta "un fort bel échantillon avec sa contre-partie, d'une espèce d'hibiscus très bien conservé» qu'il espérait pouvoir faire identifier par ses collègues botanistes du Muséum d'histoire naturelle. Le soir, Faujas alla voir le pont de Castelvecchio occupé par les troupes françaises. Le 22 octobre, il visita le Muséum d'antiquités qu'avait formé l'écrivain et amateur d'art Scipione Maffei (1675-1755). Le lendemain, Gazola emmena Faujas de Saint-Fond voir la fabrique de salpêtre qui avait été construite par l'ancien gouvernement de la République de Venise et qui était depuis sous sa direction. Tout le reste de la journée du 23 octobre, Faujas commença l'inventaire des poissons fossiles offerts par le collectionneur véronais et la préparation des caisses pour leur transport. Ce travail l'occupa pratiquement sans relâche jusqu'au 30 octobre 1805. Il était assisté d'un menuisier et de deux domestiques.

Faujas devait trouver un moyen sûr pour faire acheminer les caisses jusqu'à Milan. Le 24 octobre, il s'entretint à ce sujet avec Henri François Marie Charpentier (1769-1831), chef d'étatmajor de l'armée, dont le quartier général était situé à Alpo, à une dizaine de kilomètre au sud-ouest de Vérone. Charpentier lui promit de lui «donner toutes les facilités pour faire conduire et escorter» la précieuse cargaison de poissons fossiles, mais c'était sans compter sur les évènements qui se précipitèrent les jours suivants. Le 25 octobre, Faujas travailla durant 13 heures au classement et à l'emballage des poissons. Il ne prit qu'une pause de deux heures pour se restaurer et se détendre. Gazola lui fit visiter la maison qu'il avait mise à la disposition de Louis XVIII pendant son exil, juste avant l'entrée des troupes françaises à Vérone en 1796. Faujas décrivit ainsi cette journée:

25 [octobre 1805]. Je n'ai pas discontinué de toute la journée à inventorier, à pareiller et metre [sic] en ordre les poissons fossiles, avec deux hommes qui m'aident et un menuisier qui est à ma disposition. J'ai travaillé depuis sept heures du matin sans relache, jusqu'à trois heures. Imédiatement après le dinné, je suis allé faire une promenade pour me delasser, à une extrémité de la ville avec $\mathrm{Mr}$ Gazola et à une maison fort agréable, avec un très vaste jardin en bonne production de vignes, d'arbres à fruits de diverses espèces et en excellents potages. La maison est agréable et commode. C'est la même qu'occuppat [sic] long-tems, Monsieur [titre qu'il portait en qualité de frère cadet de Louis XVI], pendant son long séjour à Vérone; Mr de Gazola lui avait livré cette maison. Il m'a dit qu'il y menait une vie extrêmement retirée et très modeste, qu'il s'y occuppait sans cesse de lire et d'écrire, ne voyait presque personne, qu'il était très bien, et était très affecté des grands malheurs de sa famille. Je suis rentré à cinq heures pour me remetre [sic] au travail jusqu'à dix heures du soir (ADI, J 546).

Faujas de Saint-Fond notait également qu'il y avait à Vérone d'autres cabinets d'histoire naturelle qui possédaient des poissons fossiles comme celui de Gian Giacomo Dionisi et du marquis de Canossa, dans lequel les commissaires français avaient d'ailleurs réquisitionné, en 1797, 15 poissons et cinq plantes du Monte Bolca pour le Muséum national d'Histoire naturelle (Frigo \&
Sorbini 1997: 15; Gaudant 2011a: 88). Faujas mentionnait dans ses notes l'abbé Manzatti qui dessinait «avec beaucoup de vérité et de talent les poissons fossiles". Leonardo Manzati est l'un des artistes, avec Giuseppe Buffetti, qui dessina les planches de l'Ittiolitologia Veronese (Volta 1796-1809) (Girardi 1997). Le 26 octobre 1805, Faujas consigna dans son journal de voyage l'épisode de la mission de Bosc à Vérone en 1803 et la responsabilité de Chaptal dans son échec.

26 [octobre 1805] Je n'ai pas discontinué de travailler depuis sept heures du matin jusqu'à six heures du soir pour inventorier les poissons et les faire emballer. Comme ce travail exige beaucoup de soin, et de précaution, et qu'il a fallu refaire en entier ce que Bosc avait fait parce que lorsqu'il se présenta en l'absence de Mr de Gazola qui était à Paris, pour faire emballer le Cabinet de ce dernier, en vertu d'un arretté [sic] de Chaptal alors ministre de l'intérieur, qui n'avait aucun droit de prendre des arrettés imperatifs dans le republique cysalpine, les frères de Mr Gazola qui logent avec lui dans sa maison de Verone, justement indigné de ce que le don gracieux de leur frère, les metoient dans le cas d'éprouver des desagréments, et des injonctions de la part de Chaptal [car ce n'était nullement de la faute de Bosc] mirent de coté tout ce qu'il y avait de plus rare dans le cabinet de leurs frères qui est très grand et occupe plusieurs pièces; et cédant aux circonstances, ils abandonnèrent à Bosc les objets d'un plus grand volume, et ceux qui ne leur parurent pas digne d'être conservés. Tout fut ensuite renvoyé de Millan [sic] où Bosc avait envoyé ce qu'il avait choisi pour la France et Bonaparte lui-même ordonna noblement le renvoi. Mr de Gazola, qui fit ouvrir les caisses, constata les dégas occasionnés par les transports sur quelques poissons d'un grand volume, qu'il a fait réparer; et lorsque je suis arrivé, il m'a fait voir les poissons véritablement rares qu'il destinnait pour le Muséum, et toutes d'espèces nouvelles qui manquent à la collection de Paris, et qui l'enrichiront de telle sorte qu'il n'existera pas dans le monde un choix d'objets pareils, qu'on ne saurait se procurer ailleurs, ni pour or, ni pour argent et qui est le fruit de plus de quarante ans de recherches, non seulement de la part de Mr de Gazola, mais encore de ceux dont il avait précédament [sic] acquis les collections (ADI, J 546).

Du 27 au 29 octobre 1805, Faujas travailla à l'emballage des poissons pratiquement sans interruption si ce ne fut que pour aller voir, le 28 octobre, le préfet de la municipalité afin d'obtenir l'autorisation que les caisses fussent scellées (Fig. 16B). Faujas voulait en effet éviter qu'un agent des douanes zélé vint à ouvrir et fouiller sans ménagement la précieuse cargaison durant son transport jusqu'à Milan. Le 30 octobre, Faujas écrivait que toutes les caisses étaient "terminées, clouées, entourées de cerceau en bois de chataignier, les $n^{\circ}$ et les adresses écrites, plombées par les employés de la douane» et qu'il avait réglé toutes ses dépenses. Coup de théâtre, la guerre venait tout juste d'éclater à Caldiero, à une petite vingtaine de kilomètres à l'est de Vérone sur la route 
de Vicence. Impossible dans ses conditions de trouver des voitures pour transporter les poissons fossiles. Elles étaient en effet toutes réquisitionnées par l'armée pour acheminer du matériel et des vivres. Ayant appris que l'empereur Napoléon Ier venait de remporter la bataille d'Ulm contre les Autrichiens et qu'il se dirigeait vers Vienne, Masséna venait en effet de déclencher une offensive contre l'armée de l'archiduc Charles d'Autriche, qui s'était retirée sur les hauteurs de la ville de Caldiero. Le lendemain, Faujas écrivait dans son journal:

ler novembre [1805] L'armée a trouvé de grandes résistances, devant les formidables redoutes que le prince Charles a fait construire à Caldiero, à quatre lieues de Vérone, et à l'entrée du Vicentin. La première attaque n'a pas réussi, et notre armée a eu un grand nombre de blessés. Elle a pris ses positions, non loin des redoutes, et va aviser à un moyen de les cerner et de les bloquer, puisqu'elles sont imprenables par leur position et par limmense artillerie qui les défend. Elles ne sont d'ailleurs dominées par rien ce qui les rend très difficiles (ADI, J 546).

Le 2 novembre, Faujas était encore bloqué à Vérone. Le prince Charles d'Autriche avait envoyé une division près de la ville de Vérone qui n'était alors défendue que par quelques soldats Français. À cinq heures du matin, le professeur du Muséum entendit des coups de feu. Il fut même témoin de fusillades entre des soldats monténégrins de l'armée autrichienne et quelques tirailleurs français restés en position sur le pont du Castelvecchio. Faujas demanda à nouveau, mais sans succès, au préfet de la ville des voitures pour faire partir de toute urgence les caisses pour Milan. Il parvint à trouver un transporteur, un certain Locatelli, qui accepta de se charger de cette mission. Locatelli, homme de confiance, était recommandé par François Mami, le fondé de pouvoir de Gazola à Paris. Il était cependant impossible de partir le jour même vu les circonstances. Un officier français vint même chercher Faujas pour l'exhorter à fuir Vérone devant le danger imminent d'une attaque autrichienne, mais il refusa. Faujas eut raison de ne pas céder à la panique car en début d'après-midi, la nouvelle se répandit que l'archiduc Charles d'Autriche avait évacué Caldiero. Les échauffourées qu'il avait ordonnées à Vérone n'avaient pour objectif que de couvrir sa retraite qui avait commencé dès le $1^{\text {er }}$ novembre. L'armée autrichienne en Italie était en effet appelée de toute urgence pour tenter de protéger Vienne, menacée par les troupes de Napoléon Irer. Ralentie par les troupes de Masséna qui continuaient à la harceler, elle ne put participer à la bataille d'Austerlitz, qui se solda par la victoire des Français, le 2 décembre 1805, et la capitulation des Autrichiens. Ces évènements aboutirent à la signature du traité de Presbourg, le 26 décembre 1805, par lequel l'Autriche abandonnait la Vénétie orientale au Royaume d'Italie. Vérone était alors dans sa totalité sous domination française. Le récit que donne Faujas de la fameuse journée du 2 novembre 1805 mérite d'être reproduit ici pour illustrer le sang-froid dont il fit preuve:
2 [novembre 1805] $A$ cing heures du matin fusillades à deux pas des murs de Veronetta. Le prince Charles avait envoyé la division Sommariva [général Hannibal Sommariva (1755-1829)], par le haut des montagnes pour fondre sur Véronne qui n'était pas deffendue [sic] l'armée étant à quatre lieues en avant, le même prince a fait faire en même tems une autre attaque du côté de Ronco, de manière que l'armée craignant d'être cernée, a fait évaquer les équipages, les caissons, et la grosse artillerie sur Vicence, ce qui a grandement allarmé les Veronnais, qui ne [sic] me paroissent en général amis des français. Je me suis lavé et je suis sorti par la porte de Castel Vechio, pour voir la fusillade, $j$ 'ai vî de près les Montenegrins qui descendoient des montagnes voisines de la petite Verone, et que nos tirailleurs de la 22ème avoient bien de la peine à retennir parce quill neetaient pas en nombre; aussi les Montenegrins s'approchaient-ils, et sills n'avoient pas craint de tomber dans quelquiembuscades, ils auroient pu nous faire beaucoup de mal et occasionner un grand desordre dans la ville; jai resté [sic] témoin du combat, jusqu'à dix heures et demi où le feu des Montenegrins s'est tû̀, et ils se sont portés à une petite distance sur les hauteurs, tout près de Véronne, où ils sont restés dans l'attente. Pendant ce tems, nos équipages non seulement entroient dans la ville, mais plusieurs passoient en avant du côté de Mantoue. Je suis allé à la municipalité et chez le preffet pour demander deux voitures pour faire partir les caisses d'histoire naturelle destinnées pour le Muséum, mais toutes les voiture étoient en réquisition, soit pour transporter les blessés, que pour porter des vivres à l'armée. Les ponts étoient fermés de nouveau. Je suis bien déterminé à ne pas abandonner un moment les caisses; j'ai pris le parti d'aller chez un commissionnaire fort honête et très ami des français, $\mathrm{Mr}$ Locatelli qui fait toutes les commissions de Mr Mami, je lui ai demandé sil pouvait répondre de l'envoi; je m'en charge m'a-t-il dit quoi quil en arrive; il est impossible qu'elles partent dans le moment; mais je vais vous en donner mon chargement par écrit; ce qui fut dit fut fait; je fis transporter toutes les caisses chez lui, il meen donna un reçu et je lui annonçait que quoi quil arrivera, je ne quitterai pas Véronne, que les caisses ne fussent à moitié chemin de Milan et que jen ai eu la certitude. Mr Posac, officier du 30 de dragon, avec qui j'avais voyagé depuis Cremone jusqu’à Vérone, vint me dire quil me conseillait de partir de crainte que l'ennemi ne fit quelques échauffourées dans Véronne avant quil arriva [sic] des renforts; je ne vis aucune nécessité de quitter Veronne etjyy restai. A deux heures il se répandit quelque bruit que le prince Charles avait évacué les fameuses redoutes et que l'attaque qu'il avait faite avec tant de vigueur avant le jour étoit pour former une grande diversion, et favoriser la retraite de manière à conserver tous les équipages de son armée et surtout sa nombreuse artillerie; cétoit là une fort belle opération militaire de sa part. Il paroit quil avoit reçu depuis peu un courrier qui lui annoncent les défaites de l'armée autrichienne et l'ordre de fuir en retraite de peur d'être couppé par une armée française qui pourrait venir par le Tyrol (ADI, J 546). 


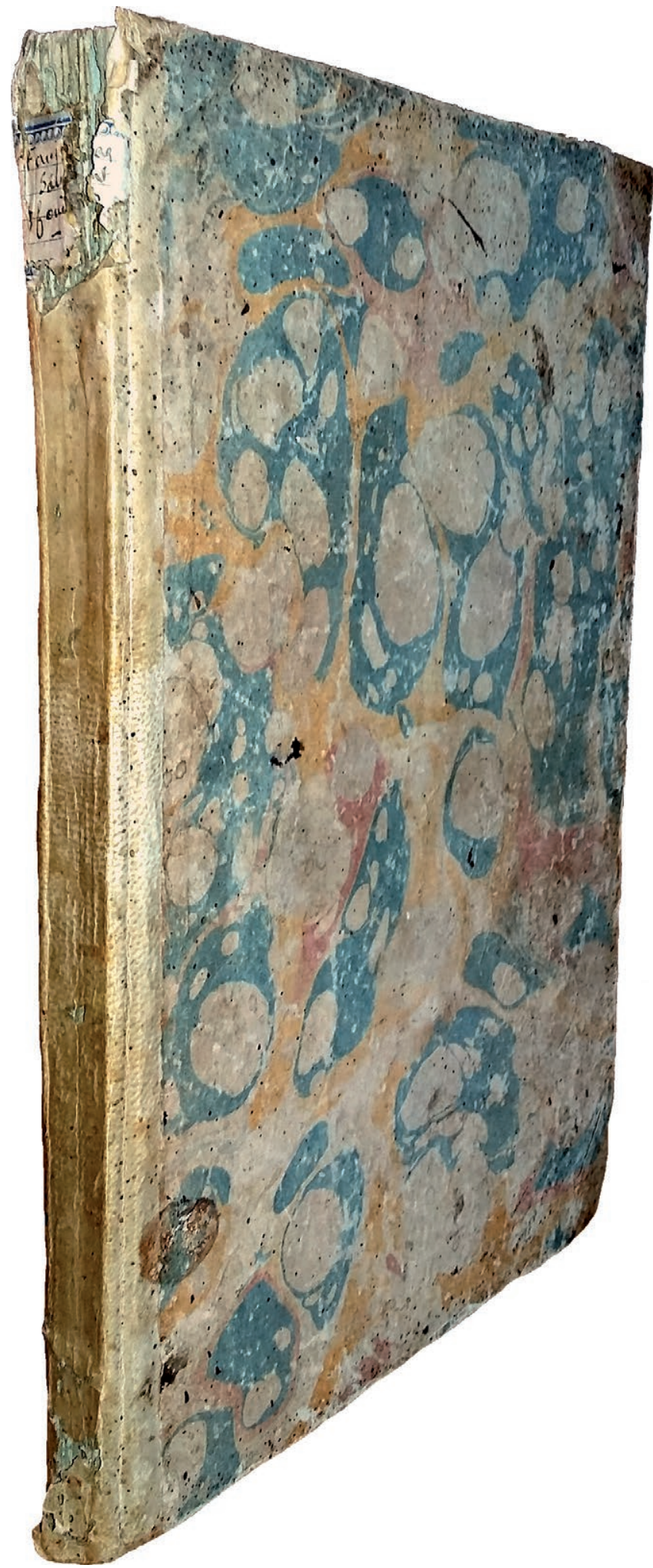

FIG. 16. - A, Journal de voyage de Barthélémy Faujas de Saint-Fond en Italie du 12 octobre au 19 novembre 1805. (C) Archives départementales de l'Isère, Grenoble (J 546).

Le 3 novembre 1805, Faujas de Saint-Fond partit à huit heures du matin en voiture avec Gazola pour aller visiter Caldiero et la place forte qui venait d'être abandonnée par l'armée autrichienne. "La plupart des morts étoient encore sur le formidable champ de bataille, qui offrait un tableau très lugubre»; "rien ne rend la guerre odieuse comme un pareil tableau» écrivit Faujas dans son journal. La région était redevenue calme et les caisses de poissons fossiles purent enfin quitter Vérone, le 5 novembre 1805, pour Milan. De son côté, Faujas de Saint-Fond continuait son voyage en Vénétie. Le 5 novembre, il repassa à Caldiero pour y effectuer des observations géologiques. Entre le 6 et le 11 novembre, il visitait Vicence et ses environs. Les 12 et 13 novembre, il était à Montecchio Precalcino et le 14, à Thiene. Le 15 novembre, Faujas visita, à Schio, le cabinet d'histoire naturelle de Berettoni qui renfermait «un choix peu nombreux, mais parfait des poissons fossiles de Vestena Nova". Après avoir visité les vallées de Mercanti et de Zuccanti, il retourna, le 17, à Vicence. Le journal de voyage de Faujas de Saint-Fond se termine le 19 novembre avec la visite de Montecchio Maggiore. La veille, lorsqu'il était encore à Vicence, il rencontra Francesco Orazio Scortegagna qui lui présenta une brochure tout juste publiée sur un requin qui avait été découvert au Monte Bolca (Scortegagna 1805; Guerra 2017). Faujas de Saint-Fond raconte dans son journal que ce fossile donna lieu à une contestation de la part de Gazola qui prétendait «que ceétoient les ouvriers qu'il employait à la carrière, qui avoient trouvé le magnifique poisson; qui devoit lui appartenir, que personne d'ailleurs n'avoit l'aurorisation d'exploiter les carrières où l'on trouve des poissons, que lui Gazola et la maison Maffei en vertu des patentes qu'ils avoient obtenus de l'ancien gouvernement de Venise. L'inspecteur des mines de l'empereur d'Allemagne soutenait que le poisson n'avoit point été trouvé dans les carrières de Gazola, ni de Mattei, mais dans une partie de montagne voisine des carrières privilégiées à la vérité, mais qui se trouvoit dans le territoire Vicentin appartenant à l'empereur d'Allemagne à cette époque. L'archiduc Jean [Jean-Baptiste d'Autriche (1782-1859)] étoit allé dans le même tems visiter Monte Bolca, comme un objet très curieux, l'inspecteur des mines de son frère l'empereur [François Ier d'Autriche (1768-1835)], étoit charmé de lui faire hommage de ce superbe objet d'histoire naturelle, mais aussitôt que le prince fut instruit de l'objet de la contestation, il se fit une délicatesse d'accepter le poisson, qu'il destinoit pour le muséum de Vienne. Le sens de la guerre ayant repoussé les autrichiens de Vicence, le poisson a été acquis par la municipalité qui l'a fait poser dans la bibliothèque publique de Vicence comme un des plus beaux monuments géologiques qui existe. Mr Scortegagna me mit au fait de tous les détails et m'assure qu'il avoit été sur les lieux et que le poisson n'avait point été découvert dans la carrière de $M^{r}$ de Gazola, ni dans celle de Mr Maffei, mais dans celle appartenant à un nommé Jean Marie Rizoni carrieur de son métier, ayant une possession dans le territoire de Bolca; que cet homme avait travaillé autrefois pour le compte de $M^{r}$ de Gazola et qu'il s'était brouillé avec lui, parce que ce dernier exigeoit que Rizoni ne travailla point dans d'autres carrières $M r$ de Gazola s'appuiant sur le droit exclusif, qu'il avoit ainsi que $M^{r}$ Maffei, d'exploiter les carrières, même celles qui n'étoient pas dans sa propriété. Quand [sic] à Mr Maffei, il ne prit aucune part à la querelle» (ADI J 546). Le spécimen en question fut également étudié par Lioy (1865) mais fut détruit durant la Seconde Guerre Mondiale (Cappetta 1975: 288, 289; Marramà et al. 2017). 


\section{yerone a}

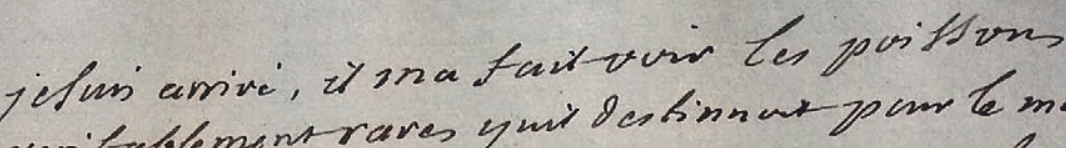
ventablementraves yuit destinnat parr te mateium,

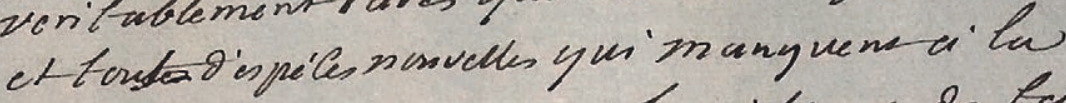
Collection depan', dyuifiennichinss de balle

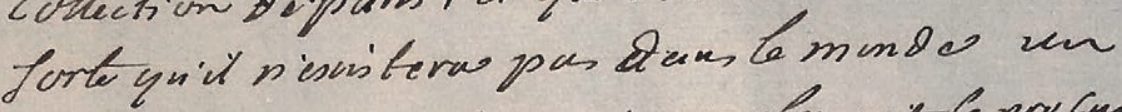

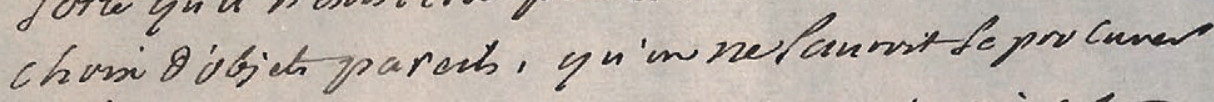

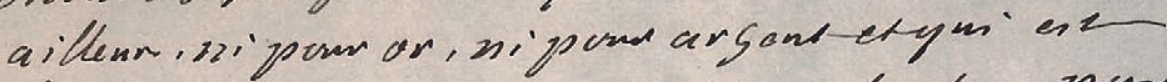
Lefinst 8 epphes 20 ipuarante ans 80 racherche. in ch Jentemerr oclupare de in-de Gazolu, in as

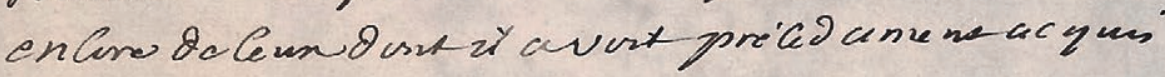
ter Corlection

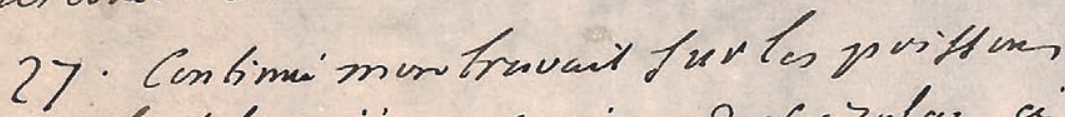
foltiter íai engage m-8c gazula a envuger yualyues portions incienara Oes rester oo K'elephane ynd De Cuant ci ruonasnano ct it mafuit esperar quid ad hevenst cimademanse

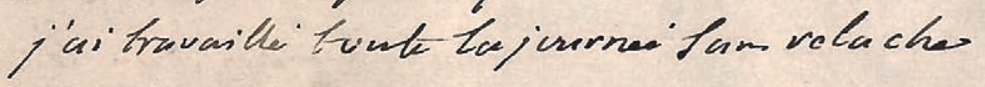

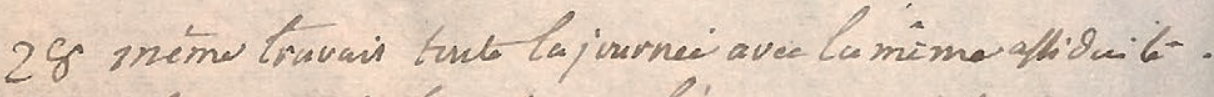

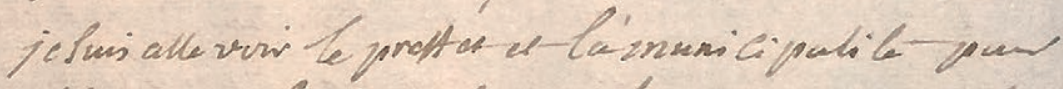
obtemin que is Casthes frien pitumber es num istilies

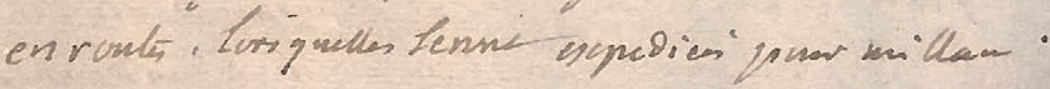

29. jai en cors Continne le mime Invait faes prens ne aucun Delostlemew

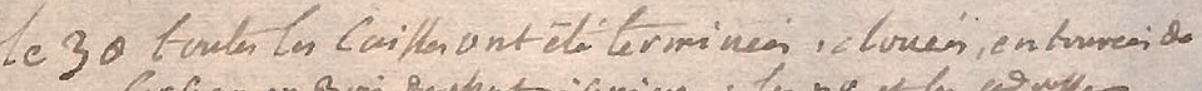

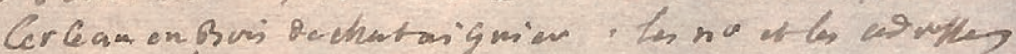

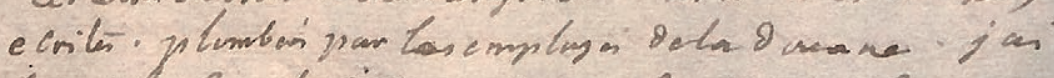

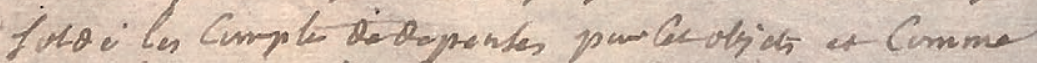

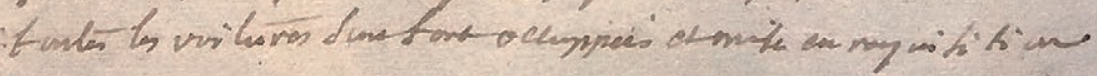

FIG. 16. - Suite. B, Une des pages du journal relative aux journées du 26 au 30 octobre 1805, alors qu'il mettait en caisse la collection de poissons du Monte Bolca offerte par Gazola. (C) Archives départementales de I'Isère, Grenoble (J 546). 


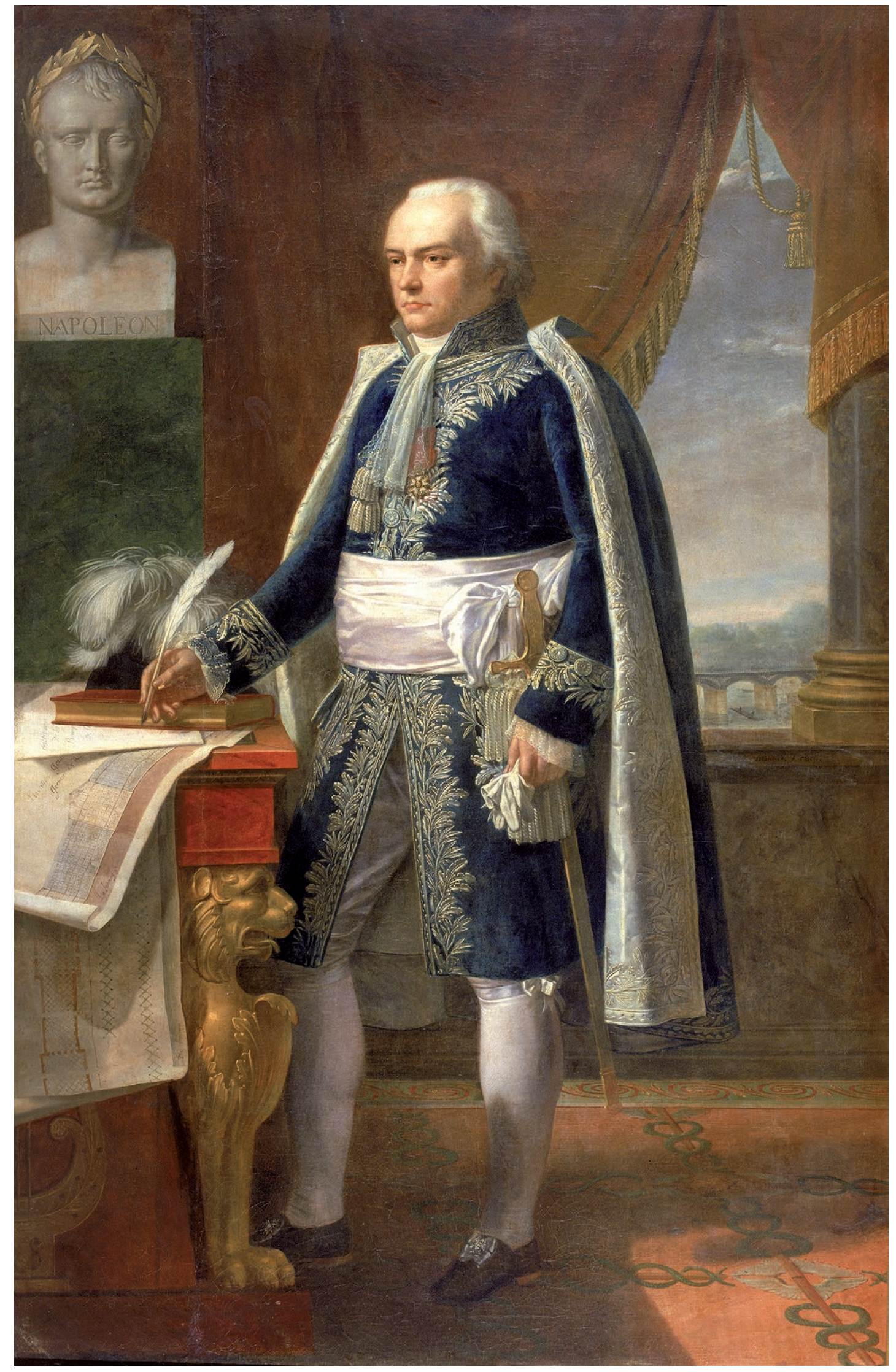

FIG. 17. - Emmanuel Crétet, comte de Champmol (10 février 1747, Pont-de-Beauvoisin, Savoie - 28 novembre 1809, Paris) par Antoine Ansiaux (1764, Liège 1840, Paris), huile sur toile, 1810. (C) Musée des Beaux-Arts de Dijon/François Jay, inv 4694. 
Secrétarian général.

Pour ledecotarnt gaiviral
Extrait de la feuille du travail de Son Excellence avec Sa Majesté l'Empereur et Roi, en date du 21. 7 Gre 1808.

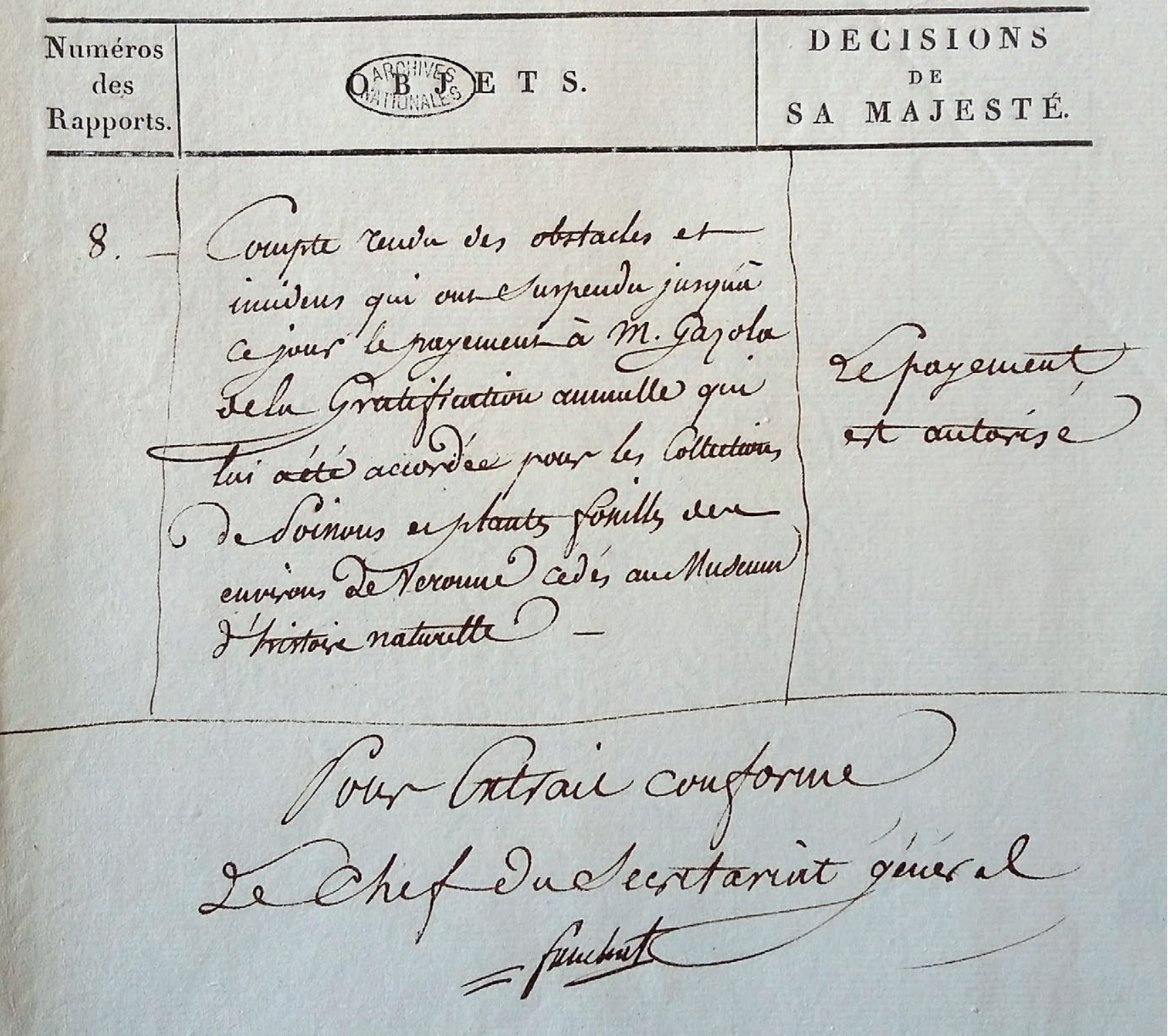

FIG. 18. - «Extrait de la feuille du travail de son Excellence» le ministre de l'Intérieur, Emmanuel Crétet, comte de Champmol, «avec Sa Majesté l'Empereur et Roi », Napoléon ler, en date du 21 septembre 1808 dans lequel sont exposés «les obstacles et incidens qui ont suspendu jusqu'à ce jour le payement à M. Gazola de la gratification annuelle qui lui a été accordée pour les collections de poissons et plantes fossiles des environs de Veronne cédés au Muséum d'histoire naturelle". Le document fait état de la décision de l'empereur d'autoriser le paiement. @) Archives nationales (F/17/1537, sous-dossier Gazola).

À la fin du mois de décembre 1805, Faujas de Saint-Fond était à Padoue où il rencontra le vice-roi d'Italie, Eugène de Beauharnais (1781-1824), fils adoptif de l'empereur
(Annexe 1[doc. 40]). Profitant du retrait des Autrichiens, il partit ensuite pour Venise où il séjourna jusqu'au 15 janvier 1806. Il y examina une collection de poissons de l'Adriatique 
qu'il voulait comparer aux espèces fossiles du Monte Bolca. Il espérait pouvoir regagner Milan pour organiser son retour en France, mais il apprit que la neige bloquait le col du MontCenis, qui était à l'époque le principal passage pour franchir les Alpes. Il décida en conséquence de poursuivre son voyage dans le Tyrol, en Styrie et en Carinthie, où il continua à récolter des échantillons de roches, de minéraux et de fossiles pour sa collection et celle du Muséum (Annexe 1[doc. 41]). Le 12 mars 1806, il arriva enfin à Milan (Annexe 1 [doc. 48]). Là l'attendaient, depuis cinq mois déjà, les caisses contenant les poissons fossiles de la collection Gazola, qu'il avait fait transporter depuis Vérone, et auxquelles s'ajoutaient les deux grandes caisses renfermant les spécimens volumineux restées en dépôt à Milan depuis le départ de Bosc en septembre 1803 (Annexe 1[doc. 47]). À celles-ci s'ajoutaient d'autres caisses remplies des spécimens collectés par Faujas au cours de son voyage, des livres qu'il avait achetés pour sa bibliothèque, une caisse de livres que le sénateur André Joseph Abrial l'avait chargé de transporter à Paris et enfin une caisse contenant des insectes et des minéraux collectés par Bosc, durant son voyage en Italie dans le courant de l'été 1803, que ce dernier avait oublié à Milan lorsque son convoi fut arrêté par ordre du Premier Consul (Annexe 1[doc. 47]). L'ensemble de la cargaison comprenait au total 23 caisses. Le vice-roi d'Italie avait proposé de mettre à la disposition de Faujas un grand caisson militaire pour transporter le tout (Annexe 1[doc. 41]). Des ordres en ce sens avaient même été donnés au chef d'état-major de l'armée, le général Henri François Marie Charpentier et le commissaire ordonnateur Louis Joubert (1762-1812). Mais tous les caissons militaires et les chevaux de l'armée étaient partis pour la Dalmatie lorsque Faujas de Saint-Fond arriva à Milan. Il dut en conséquence faire appel aux frères Soresi, deux banquiers milanais, qui se chargeaient ordinairement des envois vers la France.

Faujas voulait à tout prix éviter que les caisses ne fussent ouvertes par la douane à leur passage à la frontière et fouillées sans précaution. Il partit pour Verceil, qui était alors le cheflieu du département français de la Sésia, où se trouvait le bureau de la douane française, pour se renseigner sur la marche à suivre. Cette exemption de fouille et de droits de douane, ne pouvait être accordée que par ordre du directeur des douanes de l'empire en personne, Jean-Baptiste Collin de Sussy (1 er janvier 1750, Sainte-Menehould - 7 juillet 1826 , Paris). De Turin, Faujas écrivit au ministre de l'Intérieur, Jean-Baptiste Nompère de Champagny, le 23 avril 1806, pour le prier d'agir en conséquence (Annexe 1[doc. 42]). Faujas demanda également le soutien du général Jacques-François de Menou (1750-1810), qui était alors "Commandant général des Départements au delà des Alpes» (Annexe 1[doc. 43]). Collin ne pouvait que donner son accord à cette requête exceptionnelle, s'agissant, rappelons-le, d'un cadeau de la ville de Vérone pour l'empereur. L'ordre fut donc donné, dans le courant du mois de mai 1806 (Annexe 1[doc. 46]), au préfet de la Sésia et au directeur des douanes de Verceil de laisser passer la fameuse collection de poissons fossiles du Monte-Bolca et tout le reste de la cargaison (Annexe 1[docs 44, 45]). À la frontière, toutes les caisses furent scellées par du plomb pour que les douaniers de Paris pussent vérifier leur contenu à leur arrivée au Muséum (Annexe 1[doc. 46]). Les frères Soresi se chargèrent de l'acheminement de la cargaison jusqu'à Lyon et, de là, un autre roullier de confiance fut mandaté pour assurer son transport jusqu'à Paris. La collection Gazola 2 arriva enfin au Muséum d'Histoire naturelle, à Paris, vers le mois de juin 1806. Ceci étant dit, faute de place, la collection était toujours entreposée dans ses caisses en septembre 1808, comme l'écrivait Georges Cuvier (1769-1832) et Nicolas Louis Vauquelin (1763-1829) au ministre de l'Intérieur de l'époque (Annexe 1[doc. 53]).

De son côté, Faujas de Saint-Fond était parti de Turin et avait traversé à pied le col du Mont-Cenis, qui était encore recouvert de neige. Victime de la réverbération du soleil, il contracta une grave ophtalmie qui affectait toujours sa vue plus d'un mois après. Il arriva vers le 10 mai dans sa propriété de Saint-Fond à Loriol, où il se reposa plusieurs semaines avant de rejoindre Paris au début du mois de juillet 1806. Faujas n'avait touché qu'une seule indemnité pour couvrir les frais de sa mission de 1805. Il dut encore batailler avec le ministre de l'Intérieur pour que lui fût versée une seconde indemnisation en remboursement de ses frais de voyage pour l'année 1806. Forçant quelque peu le trait, notamment par rapport au récit des faits qu' il donne dans son journal personnel (ADI, J 546), il écrivait au ministre qu'il avait récupéré la collection de poissons fossiles au péril de sa vie: "je m'exposai plus d'une fois pour sauver cette collection, et la garantir des bombes et des obus que le prince Charles lançoit sur la ville de Verone, lorsque notre armée le força à la retraite, et passa l'Adige» (Annexe 1[doc. 48]). Les professeurs administrateurs du Muséum soutinrent également la cause de leur collègue auprès du ministre (Annexe 1[docs 49, 50]). Son indemnisation pour l'année 1806, lui fut finalement accordée le 10 janvier 1807 (Annexe 1[doc. 51]).

\section{ACTE 7}

Suite de L'INDEMNiSATION DE GAZOLA ET LES HONNEURS DU Roi DE FranCE (1808-1815)

Deux années s'étaient écoulées depuis l'arrivée à Paris de la seconde collection de poissons fossiles, quand Gazola réclama, en 1808, que lui soit rétablie la pension de 2500 francs que Chaptal avait suspendue le 1 er brumaire an 12 (24 octobre

FIG. 19. - Quelques-uns des spécimens de la "collection Gazola 2 » offerte par Gazola au Premier Consul en 1803 au nom de la ville de Vérone. Cette collection, récupérée à Vérone fin octobre 1805 par Faujas de Saint-Fond, arriva au Muséum national d'Histoire naturelle vers le mois de juin 1806 . Pour chaque spécimen est mis en regard son dessin dans le catalogue de Bosc établi chez Gazola en août 1803 (AN, F/17/1537, sous-dossier Gazola): A, B, "Platyrhina" gigantea (Blainville, 1818), holotype; A, MNHN.F.BOL567a; B, catalogue Bosc $n^{\circ} 1$ (3 pieds de long $\approx 1 \mathrm{~m}$ ); C, D, Pycnodus apodus (Volta, 1809 ), syntype; C, MNHN.F.BOL94; D, catalogue Bosc n²7 (8 pouces de long $\approx 22 \mathrm{~cm}$ ); E, F, Monopteros gigas Volta, 1809, holotype; E, MNHN.F.BOL288; F, catalogue Bosc $\mathrm{n}^{\circ} 6(1,5$ pied de long $\approx 50 \mathrm{~cm}) ; \mathbf{G}, \mathbf{H}$, Goslinophis acuticaudus (Agassiz, 1835), holotype; G, MNHN.F.BOL41; H, catalogue Bosc ${ }^{\circ} 149$ (10 pouces de long $\approx$ $27 \mathrm{~cm})$; I, J, «Pegasus» volans Volta, 1809, holotype; I, MNHN.F.BOL65; J, catalogue Bosc n 128 (2 pouces de long $\approx 5 \mathrm{~cm}) ; \mathbf{K}, \mathbf{L}$, Latellagnathus teruzzii Bannikov, 2008; K, MNHN.F.BOL3; L, catalogue Bosc n96 (6 pouces de long $\approx 16 \mathrm{~cm}$ ). Échelles: A, E, $10 \mathrm{~cm} ; \mathrm{C}, \mathrm{G}, 5 \mathrm{~cm} ; \mathrm{I}, \mathrm{K}, 3 \mathrm{~cm}$. Crédit (photographies des spécimens originaux): Recolnat (ANR-11-INBS-0004) - Christian Lemzaouda, 2012. 


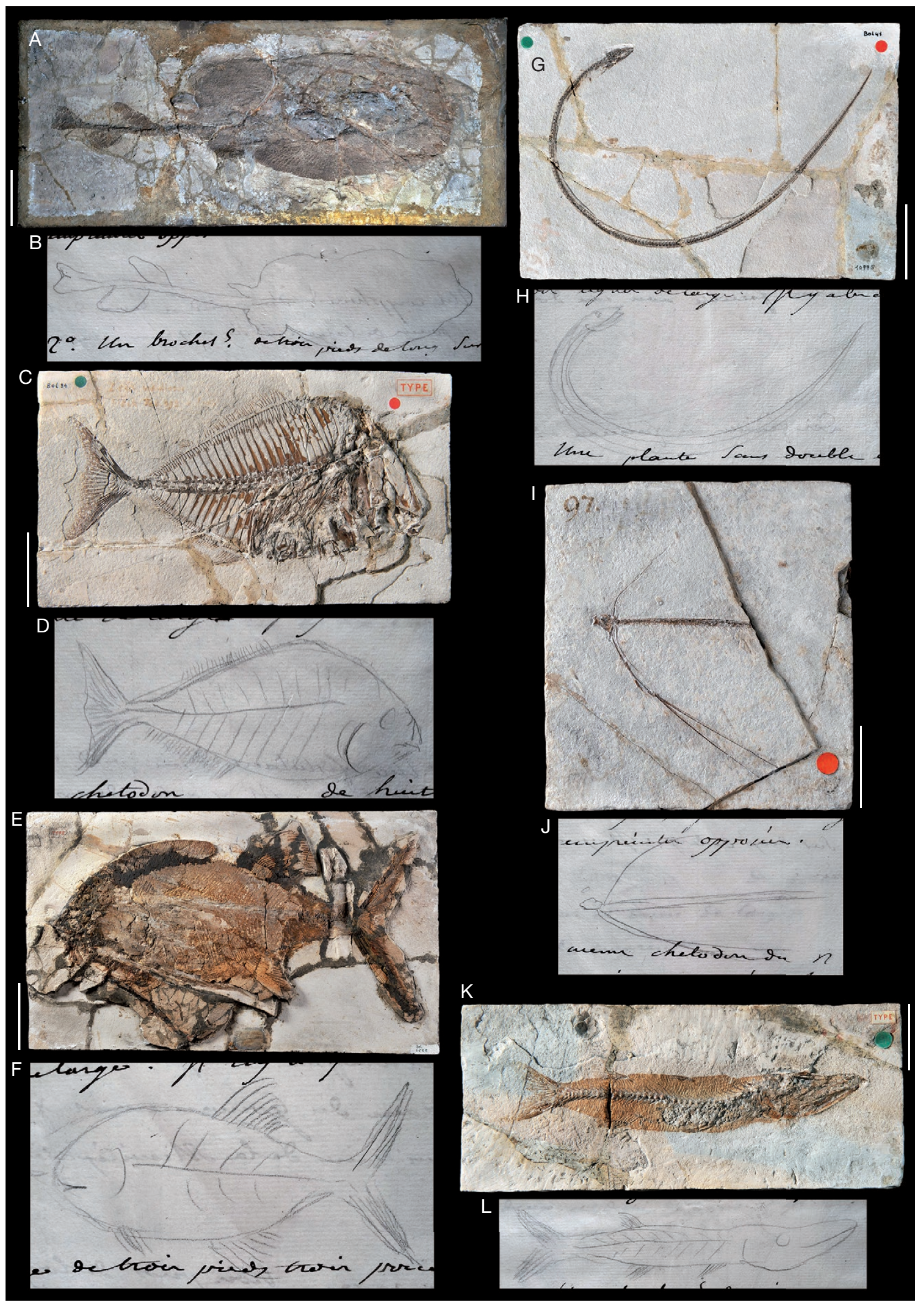


1803). Gazola demanda encore l'aide de Francesco Marescalchi, ministre des Relations extérieures du Royaume d'Italie. Le 17 août 1808, Marescalchi écrivit à l'Empereur des Français et Roi d'Italie, Napoléon Ier, en personne, pour tenter de débloquer la situation (Annexe 1[doc. 52]). L'empereur transmit le dossier à son ministre de l'intérieur, Emmanuel Crétet, comte de Champmol (Fig. 17). Né le 10 février 1747 au Pont-de-Beauvoisin en Savoie et mort le 28 novembre 1809 à Paris, Crétet était issu d'une famille de négociant qui avait fait fortune dans le commerce des étoffes (Fauchat 1809). Sous le Directoire, il fut nommé député de la Côted'Or. Bonapartiste, il soutint le coup d'État du 18 Brumaire. Bonaparte le fit nommer au Conseil d'État en 1799. Directeur général des Ponts et Chaussées de 1800 à 1806, Crétet lança une vaste politique d'aménagement et de salubrité publique dans Paris et de réfection des voies de communication dans toute la France. Premier gouverneur de la Banque de France en 1806, il fut ensuite nommé ministre de l'Intérieur le 11 août 1807, fonction qu'il quitta le 29 juin 1809 pour raison de santé.

Crétet qui découvrait l'affaire demanda un rapport aux professeurs administrateurs du Muséum d'histoire naturelle. C'est Georges Cuvier, alors directeur de cet établissement, et Nicolas Louis Vauquelin, secrétaire, qui se chargèrent de répondre à son Excellence, le 16 septembre 1808 (Annexe 1[doc. 53]). Cuvier et Vauquelin indiquèrent qu'il existait au Muséum deux collections distinctes de poissons pétrifiés du Monte Bolca, obtenues de Gazola à des époques différentes: la première acquise par Napoléon Bonaparte en 1797 et la seconde, offerte au nom de la ville de Vérone, arrivée plus récemment. Ils rappelaient que Chaptal avait nommé Bosc pour aller la chercher en Italie mais que «ce savant ayant éprouvé quelques difficultés, Mr Portalis ministre par interim, envoya par arrêté du 28 vendémiaire an XIII, Mr Faujas l'un de nous pour cet objet». Les deux professeurs avouaient ne pas connaître la valeur en numéraire de ces collections, "mais la peine qu'il a fallu pour retirer d'une carriere, des objets aussi nombreux, et pour les conserver aussi bien, tout délicats qu'ils sont, a dû exiger de grands frais». Ils concluaient que la pension de " 2400 francs dont parle Gazola» (il s'agit en fait de 2500 francs) était certainement bien inférieure à la valeur de ces précieux objets. Marescalchi envoya au ministre, le 19 septembre 1808, un second rapport qui résumait toute l'affaire (Annexe 1[doc. 54]). Il est intéressant de noter qu'il y déclare que la première collection de Gazola "lui avait été acheté [sic] ». Bosc y est encore vivement critiqué: "arrivé à Vérone, il éleva une prétention singulière; ce fut qu'on devait lui livrer la collection entière de M. Gazola, quelque fut l'état de désordre où elle était, et sans attendre le retour du propriétaire, qui pourtant l'avait exigé. Des caisses furent remplies de tous ces objets ramassés indistinctement chez M. Gazola, et dirigées sur Paris. S.M. [Sa Majesté Napoléon Bonaparte] informée de la manière dont $M$. Bosc avait rempli sa mission, ordonna que les caisses seraient arrêtées partout où elles se trouveraient et remises à la disposition de M. Gazola». Diplomate, Marescalchi faisait allusion à un malentendu qui avait fait croire à Chaptal que la collection n'avait pas été livrée et qui l'avait décidé à suspendre la pension de Gazola. Sur la base de ces éléments, Emmanuel Crétet rendit son rapport à l'empereur le 21 septembre (Annexe 1[doc. 55]). Sans le nommer, il jugeait la décision de Chaptal «mal à propos». Il concluait son rapport ainsi : "il ne peut plus y avoir aucune espèce de doute aujourd'hui sur la légitimité des reclamations soumises à Votre Majesté par son Ministre des Relations extérieures du Royaume d'Italie. Il résulte de tous ces faits que la gratification accordée à Mr Gazola doit lui être payée, et comme elle est assignée sur les fonds du Ministère de l'Intérieur, je vais prendre les mesures que me permettra la situation de ces fonds et commencer par le payement de l'année courante». L'empereur agréa cette décision (Fig. 18). La pension annuelle de 2500 francs de Gazola, en indemnisation de sa première collection, fut donc rétablie à partir de 1808. Le 7 décembre 1808, le collectionneur véronais envoya à Crétet une lettre de remerciement où il désignait Marescalchi comme son fondé de pouvoir pour toucher les sommes de l'année courante et les arriérés des années précédentes (Annexe 1[doc. 59]).

Au début de l'année 1814, les troupes de la Sixième Coalition envahissèrent le territoire français. Napoléon Ier dut abdiquer le 6 avril 1814. En Italie, le vice-roi, Eugène de Beauharnais, essayait depuis le mois d'août 1813 de contenir l'invasion autrichienne mais en avril 1814, il fut contraint d'abandonner le royaume d'Italie qui tomba sous l'occupation autrichienne (Fig. 7). Un an plus tard, le 7 avril 1815, les territoires occupés par les autrichiens en Italie devinrent le nouveau Royaume de Lombardie-Vénétie, dont la couronne fut confiée à l'empereur d'Autriche, François Ier. La ville de Vérone resta ainsi sous domination autrichienne jusqu'en 1866. En France, la Première Restauration se mettait en place avec le retour du roi Louis XVIII et l'adoption de la charte constitutionnelle du 4 juin 1814. Dans cette période de profond bouleversement politique, la pension de Gazola fut suspendue.

Le comte Giovanni Battista Gazola, qui avait accordé l'hospitalité au souverain français durant son exil, lui écrivit le 22 décembre 1814 , non pas seulement pour réclamer le rétablissement de sa pension, mais dans l'espoir d'obtenir une compensation plus conséquente (Annexe 1[doc. 60]). Dans sa lettre, Gazola rappelait opportunément son attachement «à la Famille Royale de France» et le soutien du comte Berthollet, pair

FIG. 20. - Quelques-uns des spécimens de la «collection Gazola 2 » offerte par Gazola au Premier Consul en 1803 au nom de la ville de Vérone. Cette collection, récupérée à Vérone fin octobre 1805 par Faujas de Saint-Fond, arriva au Muséum national d'Histoire naturelle vers le mois de juin 1806 . Pour chaque spécimen est mis en regard son dessin dans le catalogue de Bosc établi chez Gazola en août 1803 (AN, F/17/1537, sous-dossier Gazola): A, B, Eoholocentrum macrocephalum (Blainville, 1818), holotype ; A, MNHN.F.BOL576b ; B, catalogue Bosc $n^{\circ} 107$ (4 pouces de long $\approx 11 \mathrm{~cm}$ ); C, D, Pseudosyngnathus opisthopterus (Agassiz, 1835), holotype; C, MNHN.F.BOL123; D, catalogue Bosc $n^{\circ} 66$ (14 pouces de long $\approx 38 \mathrm{~cm}$ ); E, F, Eosphaeramia pygopterus (Agassiz, 1835), holotype; E, MNHN.F.BOL34; F, catalogue Bosc n¹44 (1 pouce de long $\approx 3 \mathrm{~cm}$ ); G, H, Paratrachinotus tenuiceps (Agassiz, 1834), holotype; G, MNHN.F.BOL407; $\mathbf{H}$, catalogue Bosc $n^{\circ} 105$ (3 pouces de long $\approx 8 \mathrm{~cm}$ ); I, J, Vomeropsis triurus (Volta, 1809), holotype; I, MNHN.F.BOL413; J, catalogue Bosc ${ }^{\circ} 58$ (7 pouces de long $\approx 19 \mathrm{~cm}$ ); K, L, Ductor vestenae (Volta, 1809); K, MNHN.F.BOL234; L, catalogue Bosc n68 (6 pouces de long $\approx 16 \mathrm{~cm}$ ). Échelles : C, I, K, $5 \mathrm{~cm} ; \mathrm{A}, \mathrm{G}, 3 \mathrm{~cm}$; E, $1 \mathrm{~cm}$. Crédit (photographies des spécimens originaux) : Recolnat (ANR-11-INBS-0004) - Christian Lemzaouda, 2012. 

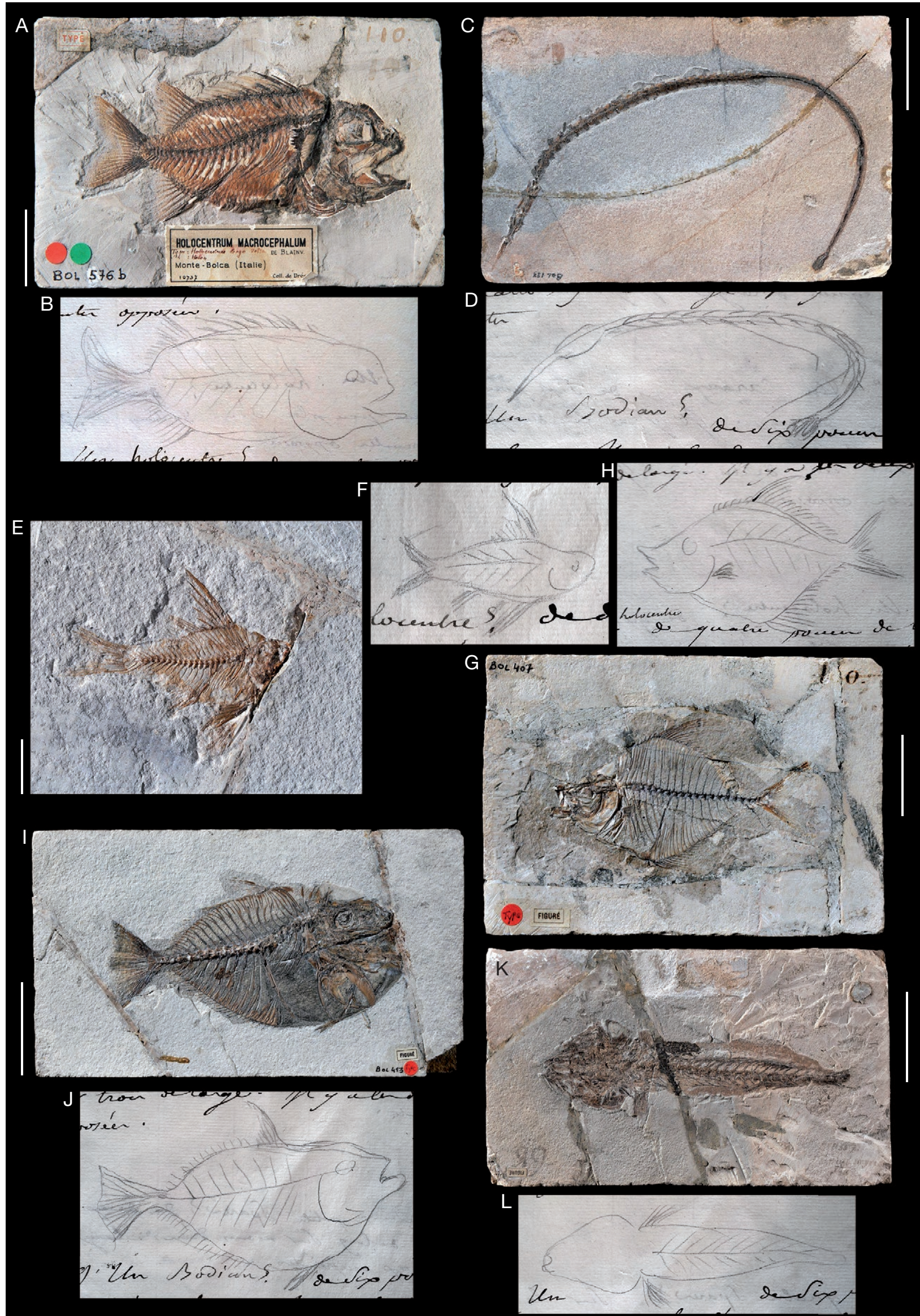
de France, qui lui avait permis d'obtenir une indemnisation pour la perte de sa première collection de poissons fossiles du Monte Bolca. Cependant, l'indemnisation annuelle qu'il avait touchée jusqu'alors s'élevait à 4800 francs correspondant au cumul des 2300 francs qu'il recevait de l'administration de Mantoue et des 2500 francs qu'il percevait du gouvernement français. Au final, cette somme n'égalait toujours pas la rente de 6000 francs qui avait été convenue en août 1797. Par ailleurs, Gazola se plaignait que l'indemnité qu'il touchait n'était que viagère et non perpétuelle comme il le souhaitait (Annexe 1[doc. 61]). En cette fin d'année 1814 et au début de l'année 1815, Gazola résidait à Paris, hôtel de Hambourg, rue Jacob. Il parvint à obtenir une audience privée auprès du roi. Louis XVIII lui donna des "témoignages les plus flatteurs d'une particulière bienveillance» et lui assura "que cette affaire serait promptement terminée et à son entière satisfaction" (Annexe 1[doc. 61]). Le roi demanda à son ministre de l'Intérieur, l'abbé de Montesquiou (1756-1832), d'instruire le dossier. Né au château de Marsan dans le Gers, le 3 août 1756, François-Xavier-Marc-Antoine de Montesquiou-Fezensac rentra dans les ordres. Il fut élu député du clergé par la ville de Paris aux États généraux de 1789 et émigra sous la Terreur en Angleterre puis en Amérique. À son retour en France après la chute de Robespierre, il s'afficha comme un partisan du parti royaliste et fut exilé à Menton par Bonaparte. Membre du gouvernement provisoire mis en place par Louis XVIII, il accepta le ministère de l'Intérieur, le 13 mai 1814, et exerça ses fonctions jusqu'au 19 mars 1815 .

Le 31 janvier 1815, l'abbé de Monstesquiou rappela que cette affaire d'indemnisation avait été jugée en 1803, qu' elle avait été confirmée en 1808, et qu'à cette époque, Gazola s'était félicité de ces arrangements. Le ministre de l'Intérieur estimait donc qu'il n'y avait plus lieu de revenir dessus (Annexe 1 [doc. 63]). Il fit néanmoins le nécessaire auprès de son collègue, le baron Joseph-Dominique Louis (1755-1837), ministre des Finances, pour rétablir la pension de 2500 francs à laquelle Gazola avait droit (Annexe 1[doc. 64]). Le 7 mars 1815, cette décision fut confirmée (Annexe 1[doc. 65]). Gazola écrivit à nouveau à Louis XVIII pour lui demander cette fois-ci de recevoir le titre de Commandant de la Légion d'honneur, en récompense de son aide lorsqu'il l'hébergea à Vérone (Annexe 1[doc. 66]). Sur ces entrefaites, Napoléon venait de quitter son exil de l'île d'Elbe et débarquait en France le 1er mars 1815. Le 19 mars, l'empereur étant aux portes de Paris, Louis XVIII et sa cour quittaient Paris pour s'installer à Gand, en Belgique. C'est donc dans cette ville flamande que fut instruite la demande de Gazola alors qu'en France, Napoléon venait de reprendre le pouvoir. Le ministre de la Maison du Roi, Pierre Louis Jean Casimir de Blacas d'Aulps (1771-1839), remit au roi, le 12 mai 1815, un rapport favorable (Annexe 1[doc. 67]) à la requête de Gazola. Ce dernier fut prévenu, le 14 mai, que le roi lui accordait "avec plaisir la décoration et le grade de commandant de la légion d'honneur" (Archives nationales, $\mathrm{O} / 3 / 841$, dossier 3).

En guise de conclusion de ce chapitre, il est intéressant d'essayer d'évaluer la somme perçue par Gazola entre 1797 et 1815 pour sa première collection déposée au MNHN en 1798. Il convient pour cela d'additionner la rente annuelle de 2300 francs qu'il touchait de l'administration de Mantoue depuis 1797 et la pension annuelle de 2500 francs qui fut mise en place par le gouvernement français en avril 1803. Même si cette dernière fut suspendue par Chaptal en octobre 1803, Crétet la rétablit en 1808 et régla les années non perçues, si bien qu'on peut considérer que cette pension fut versée sur toute cette période à partir de l'année 1803 incluse. Le montant total s'élève donc à 76200 francs sans compter ce que Gazola continua à toucher après 1815 .

\section{LES COLLECTIONS GAZOLA 1 (1798) ET GAZOLA 2 (1805) CONSERVÉES AU MUSÉUM NATIONAL D'HISTOIRE NATURELLE}

\section{Collection Gazola 1}

Comme il l'a été montré précédemment, cette collection fut d'abord confisquée à Vérone en mai 1797. Avec l'accord de Napoléon Bonaparte, des mesures furent prises pour indemniser Gazola par l'octroi d'une rente annuelle d'environ 2300 francs à partir de septembre 1797 à laquelle s'ajouta une pension annuelle de 2500 francs à partir de 1803. Au départ de Vérone, cette collection comprenait 516 poissons fossiles (189 doubles empreintes et 138 empreintes seules) d'après le procès-verbal de réquisition de la maison Gazola, dressé à Vérone par la Commission des Arts le 28 floréal an 5 (17 mai 1797) (Nicolis 1907: 43-44; Frigo \& Sorbini 1997; Gaudant $2011 b)$. Il est à noter qu'un certain nombre de ces poissons, déposés d'abord au Musém national d'Histoire naturelle en septembre 1798, furent distribués aux cabinets d'histoire naturelle des écoles centrales départementales (Frigo \& Sorbini 1997: 22; Gaudant 2011b: 642; Brignon 2015: 67).

\section{Collection Gazola 2}

Elle fut offerte en 1803 à Napoléon Bonaparte par Gazola au nom de la ville de Vérone. Excepté le triptyque MNHN.F.BOL583 (Fig. 13) donné le 20 floréal an 11 (10 mai 1803) alors que Gazola était à Paris, l'ensemble de cette collection fut récupéré à Vérone en octobre 1805 par Faujas de Saint-Fond et arriva au Muséum vers le mois de juin 1806. Comme l'écrivait

FIG. 21. - Quelques-uns des spécimens de la «collection Gazola 2 » offerte par Gazola au Premier Consul en 1803 au nom de la ville de Vérone. Cette collection, récupérée à Vérone fin octobre 1805 par Faujas de Saint-Fond, arriva au Muséum national d'Histoire naturelle vers le mois de juin 1806. Pour chaque spécimen est mis en regard son dessin dans le catalogue de Bosc établi chez Gazola en août 1803 (AN, F/17/1537, sous-dossier Gazola): A, B, Jimtylerius temnopterus (Agassiz, 1835), lectotype; A, MNHN.F.BOL533; B, catalogue Bosc $n^{\circ} 116$ (4 pouces de long $\approx 11 \mathrm{~cm}$ ). C, D, Goujetia crassispina (Agassiz, 1839), holotype; C, MNHN.F.BOL9; D, catalogue Bosc $n^{\circ} 79$ (5 pouces de long $\approx 14 \mathrm{~cm}$ ); E, F, Archaephippus asper (Volta, 1809); E, MNHN.F.BOL214; F, catalogue Bosc $\mathrm{n}^{\circ} 77$ (5 pouces de long $\approx 14 \mathrm{~cm}) ; \mathbf{G}, \mathbf{H}$, Archaephippus asper (Volta, 1809), holotype; G, MNHN.F.BOL219; H, catalogue Bosc $\mathrm{n}^{\circ} 139$ (3,5 pouces de long $\left.\approx 9 \mathrm{~cm}\right)$; I, J, Gillidia antiqua (Agassiz, 1835), holotype; I, MNHN.F.BOL12; J, catalogue Bosc n`95 (5 pouces de long $\approx 14 \mathrm{~cm}$ ); K, L, Pygaeus bolcanus (Volta, 1809), holotype; K, MNHN.F.BOL520; L, catalogue Bosc n¹0 (1,5 pied de long $\approx 50 \mathrm{~cm}$ ). Échelles : K, $10 \mathrm{~cm} ; \mathrm{A}, \mathrm{C}, \mathrm{E}, \mathrm{G}, \mathrm{I}, 3 \mathrm{~cm}$. Crédit (photographies des spécimens originaux): Recolnat (ANR-11-INBS-0004) - Christian Lemzaouda, 2012. 


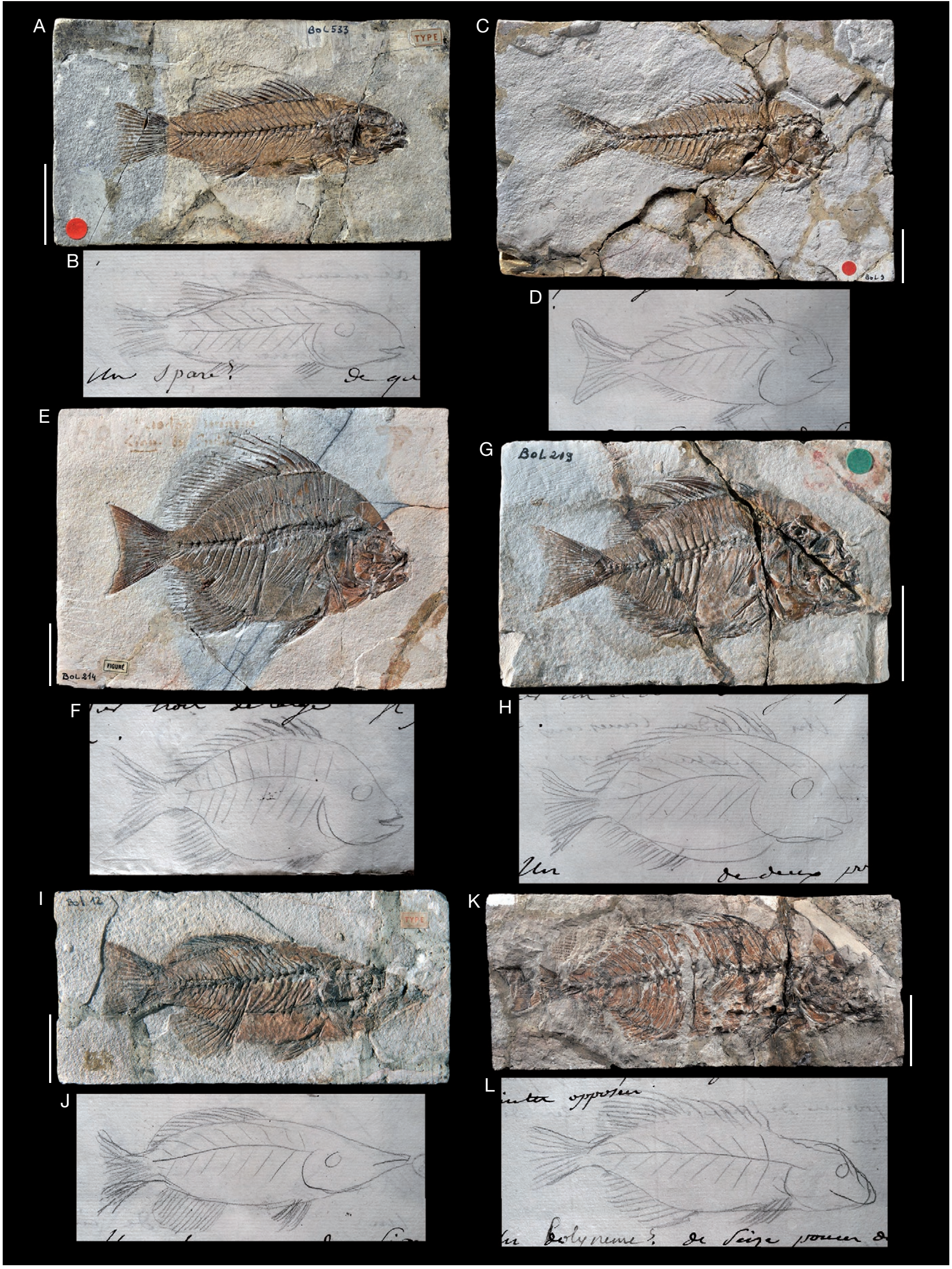


Georges Cuvier et Louis-Nicolas Vauquelin au ministre de l'Intérieur, dans une lettre en date du 16 septembre 1808 (Annexe 1[doc. 53]), cette seconde collection était tout aussi magnifique que la première, comportait cependant moins de spécimens mais contenait des pièces plus volumineuses (notamment les spécimens MNHN.F.BOL544a, BOL544b, BOL567a, BOL567b, BOL569a et BOL569b).

Plusieurs spécimens de la collection Gazola 2 ont pu être identifiés à partir des dessins effectués par Bosc chez Gazola, à Vérone, en août 1803. Sont listés ci-après quelques-uns de ces spécimens, notamment ceux qui sont figurés par Volta (1796-1809). Cette liste n'est pas exhaustive car les dessins de Bosc restent très sommaires et seuls les spécimens les mieux caractérisés ont pu être reconnus. Par ailleurs, Gazola signalait à Faujas de Saint-Fond qu'un certain nombre de beaux spécimens avaient été cachés juste avant l'arrivée de Bosc. Ces pièces ne figurent donc pas dans le catalogue de Bosc. Il est possible que certaines d'entre-elles furent données à Faujas de Saint-Fond et font en conséquence partie de la collection Gazola 2 sans qu'on puisse les reconnaître, en l'absence de figurations.

Dans ce qui suit, la taxonomie retenue par Bannikov (2014), Carnevale et al. (2014) et Marramà et al. (2017) a été suivie. Plusieurs modifications relatives à l'année de création de certaines espèces ont été cependant effectuées. Une des modifications principales concerne la révision faite par Agassiz (1835a) des espèces publiées dans l'Ittiolitologia Veronese de Volta (1796-1809). Cette révision fut publiée pour la première fois en janvier 1835 dans la 4 e livraison des "Recherches sur les Poissons fossiles» (voir Brignon 2014, pour les dates de publication des livraisons de cet ouvrage). Agassiz y introduit de nombreuses espèces nouvelles qu'il décrivit et figura dans des livraisons parues à des dates postérieures. L'année de création de 1835 est rarement retenue pour ces espèces en invoquant l'absence de description (nomina nuda). Or, dans la plupart des cas, les nouvelles espèces introduites par Agassiz en 1835 font référence aux spécimens figurés par Volta (1796-1809) et sont donc valides au regard des articles 12.1 et $12.2 .7 \mathrm{du}$ Code international de Nomenclature zoologique (ICZN 1999). Par ailleurs, plusieurs précisions sont également apportées sur le statut des spécimens types. Les espèces créées par Agassiz se fondent très souvent sur un ensemble de syntypes, parmi lesquels a été parfois désigné à tort un holotype. Enfin, il faut rappeler que la publication de l'ouvrage de Volta, Ittiolitologia Veronese, s'échelonna sur une période de quatorze ans, entre 1796 et 1809 (Gaudant 2011a). Les actes nomenclaturaux qu'il contient sont donc assez délicats à dater. En 1796, seule la première livraison était parue, contenant les pages I à LII (1 à 52) de la première partie, les pages I à LII (1 à 52) de la seconde et les planches 1 à 11 (Gaudant 2011a: 85, 86). Il est à noter que Lacépède (1798: 203, 490) fait référence, en 1798 , aux planches 3 et 8 et que Joseph Banks possédait dans sa bibliothèque, en 1799 , cette première livraison accompagnée des planches 1 à 11 (Dryander 1799: 330). Ainsi faut-il garder 1796 comme l'année de création d'Uranoscopus rastrum Volta, 1796 (= Ramphosus rastrum (Volta, 1796)), Kurtus velifer Volta, 1796 (= Exellia velifer (Volta, 1796)), Raja muricata Volta, 1796 (= Dasyatis muricata (Volta, 1796)) et Gobius veronensis Volta, 1796 (= Eocottus veronensis (Volta, 1796)). Pour toutes les autres espèces créées par Volta, l'année 1809 est à retenir (ICZN 1999: art. 21.5).

Les spécimens reconnus dans le catalogue de Bosc, qui font donc partie de la collection Gazola 2, sont:

- MNHN.F.BOL3, Latellagnathus teruzzii Bannikov, 2008 (Volta 1796-1809: pl. 24, fig. 3; Agassiz 1839a: vol. 5, pl. 38, fig. 2; Bannikov 2008b: fig. 4B). Ce spécimen est un des syntypes de Rhamphognathus paralepoides Agassiz, 1835 (Agassiz 1843b: vol. 5, 1ère partie, 104) $\AA$ catalogue Bosc n96 (Fig. 19K, L).

- MNHN.F.BOL4 et sa contre-empreinte BOL5, holotype par monotypie (ICZN 1999: art. 73.1.2) d'Eozanclus brevirostris (Agassiz, 1835) (Volta 1796-1809: pl. 26, fig. 2; Agassiz 1835a: vol. 4, pl. 38; Blot \& Voruz 1975: pls 1-2) ® catalogue Bosc n ${ }^{\circ} 133$ (Fig. 23K, L).

- MNHN.F.BOL8 et sa contre-empreinte BOL9, holotype de Goujetia crassispina (Agassiz, 1839) (Bannikov 2006: fig. 7) (B) catalogue Bosc $n^{\circ} 79$ (Fig. 21C, D).

- MNHN.F.BOL12 et sa contre-empreinte BOL13, holotype par monotypie de Gillidia antiqua (Agassiz, 1835) (Volta 1796-1809: pl. 45, fig. 1; Agassiz 1835b: vol. 4, pl. 43) ® catalogue Bosc n 95 (Fig. 21I, J).

- MNHN.F.BOL16 et sa contre-empreinte BOL15, lectotype d'Eorandallius rectifrons (Agassiz, 1835) (Volta 1796-1809: pl. 33; Agassiz 1839b: vol. 4, pl. 36, fig. 3; Blot \& Tyler 1990: $55)$. Cette espèce fut introduite de manière valide en 1835 , Agassiz (1835a: 35) se référant à la figure publiée par Volta (ICZN 1999: art. 12.1 et 12.2.7). Agassiz (1842: vol. 4, 213215) mentionnait un second exemplaire faisant partie, avec le spécimen dont il est question ici, du matériel lui ayant servi à créer Naseus rectifrons. Dans ces conditions, MNHN.F.BOL15, BOL16 ne peut être désigné comme l'holotype contrairement à l'opinion de Blot \& Tyler (1990: 55). Nous désignons donc ici explicitement MNHN.F.BOL15, BOL16 comme le lectotype de l'espèce ${ }^{\circledR}$ catalogue Bosc $n^{\circ} 28$ (Fig. 22I, J).

- MNHN.F.BOL34, holotype par monotypie d'Eosphaeramia pygopterus (Agassiz, 1835) (Volta 1796-1809: pl. 14, fig. 1; Agassiz 1837: vol. 4, pl. 9, fig. 1; Bannikov 2008a: fig. 2). Cette espèce fut introduite de manière valide en 1835 ,

FIG. 22. - Quelques-uns des spécimens de la «collection Gazola 2 » offerte par Gazola au Premier Consul en 1803 au nom de la ville de Vérone. Cette collection, récupérée à Vérone fin octobre 1805 par Faujas de Saint-Fond, arriva au Muséum national d'Histoire naturelle vers le mois de juin 1806 . Pour chaque spécimen est mis en regard son dessin dans le catalogue de Bosc établi chez Gazola en août 1803 (AN, F/17/1537, sous-dossier Gazola): A, B, Sphyraena bolcensis Agassiz, 1835; A, MNHN.F.BOL569a; B, catalogue Bosc $n^{\circ} 3$ (2,5 pieds de long $\approx 80 \mathrm{~cm}$ ); C, D, Eoplatax papilio (Volta, 1809), holotype; C, MNHN.F.BOL92; D, catalogue Bosc $\mathrm{n}^{\circ} 131$ (3 pouces de long $\approx 8 \mathrm{~cm}$ ); E, F, Exellia velifer (Volta, 1796), syntype; E, MNHN.F.BOL385; F, catalogue Bosc $\mathrm{n}^{\circ} 104(2$ pouces de long $\approx 5 \mathrm{~cm}$ ); G, H, Tylerichthys nuchalis (Agassiz 1835); G, MNHN.F.BOL110; H, catalogue Bosc ${ }^{\circ} 59$ (6 pouces de long $\approx 16 \mathrm{~cm}$ ); I, J, Eorandallius rectifrons (Agassiz, 1835), lectotype; I, MNHN.F.BOL15; J, catalogue Bosc n²8 (8 pouces de long $\approx 22 \mathrm{~cm}$ ); K, L, Xiphopterus falcatus (Volta, 1809), holotype; K, MNHN.F.BOL544a et BOL544b; L, catalogue Bosc $n^{\circ} 2$ (3 pieds de long $\approx 1 \mathrm{~m}$ ). Échelles: A, K, $10 \mathrm{~cm} ; \mathrm{G}, \mathrm{I}, 5 \mathrm{~cm} ; \mathrm{C}, \mathrm{E}, 3 \mathrm{~cm}$. Crédit (photographies des spécimens originaux) : Recolnat (ANR-11-INBS-0004) - Christian Lemzaouda 2012. 

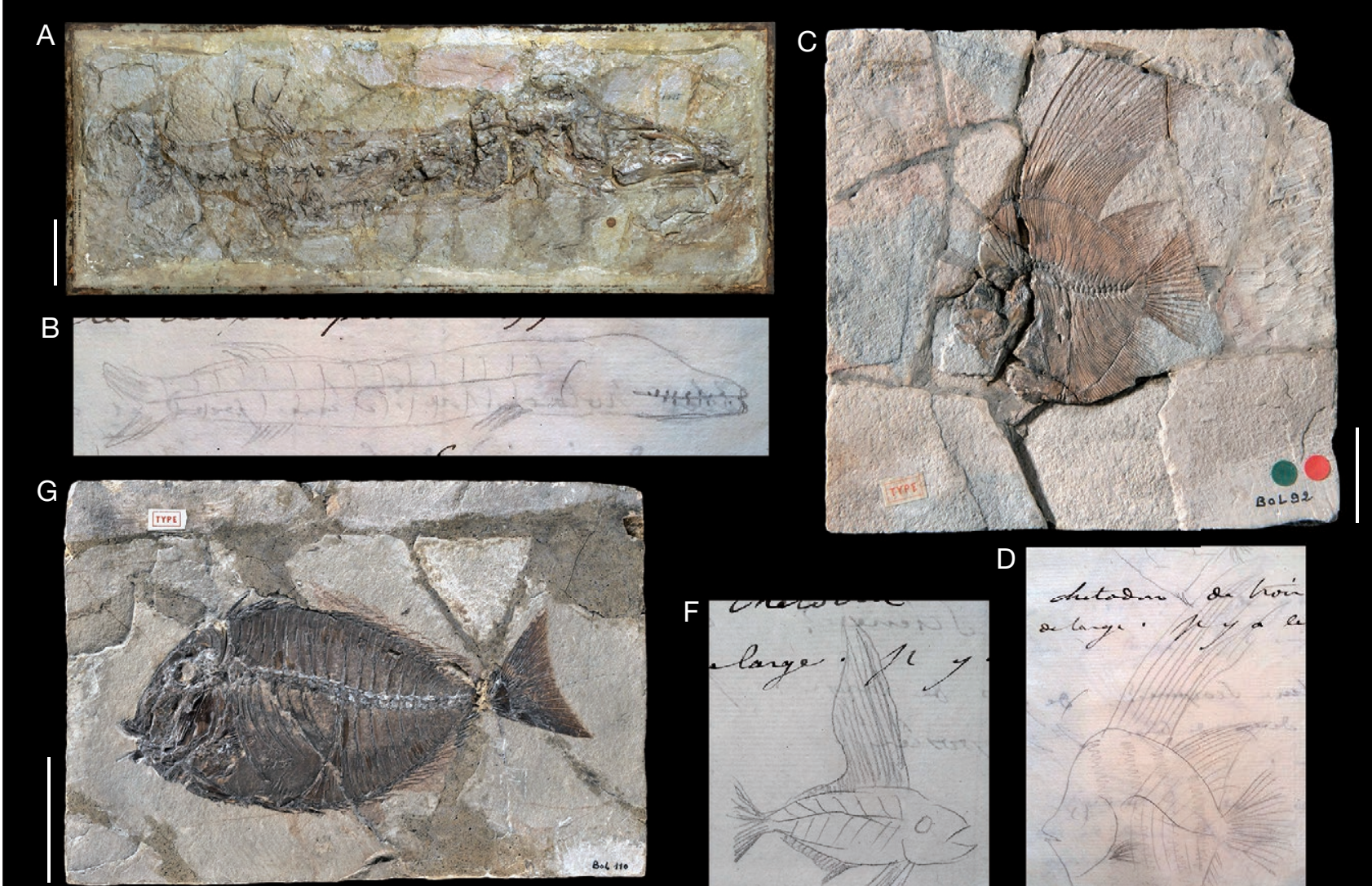

4 Hitiog
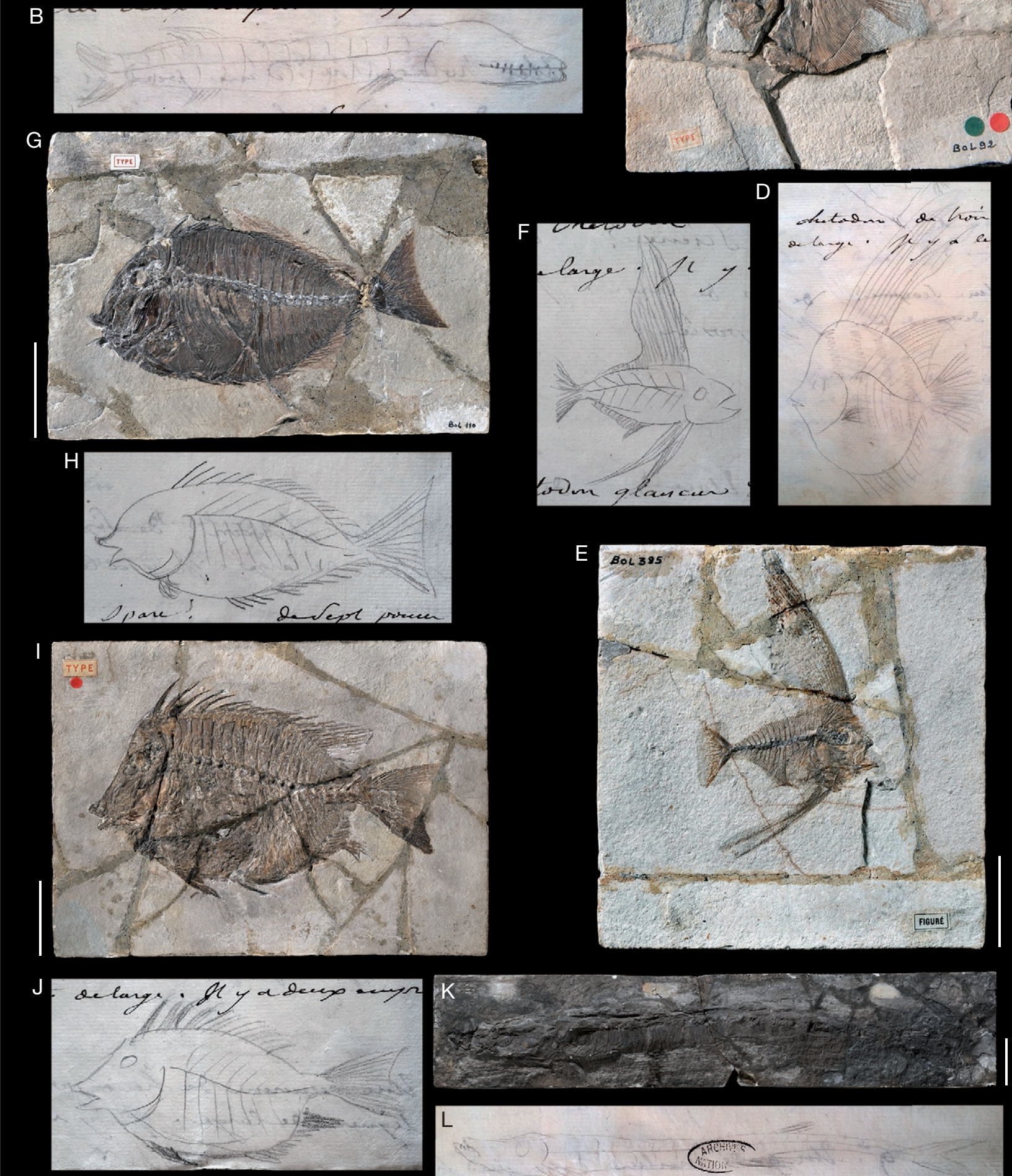
Agassiz (1835a: 34) se référant à la figure publiée par Volta (ICZN 1999: art. 12.1 et 12.2.7) ${ }^{\circledR}$ catalogue Bosc nº 144 (Fig. 20E, F).

- MNHN.F.BOL41 et sa contre-empreinte BOL40, holotype par monotypie de Goslinophis acuticaudus (Agassiz, 1835) (Volta 1796-1809: pl. 23, fig. 1; Blot 1984: 139, pls 17, 21 ; Clément 2017: 40-41). Cette espèce fut introduite de manière valide en 1835, Agassiz (1835a: 34) se référant à la figure publiée par Volta (ICZN 1999: art. 12.1 et 12.2.7) ${ }^{\circledR}$ catalogue Bosc n¹49 (Fig. 19G, H).

- MNHN.F.BOL51 et sa contre-empreinte BOL52, syntype d'Acanthonemus subaureus (Blainville, 1818) (Volta 1796-1809: pl. 19; Agassiz 1834a: vol. 5, pl. 4; Blot 1969: pl. 80-81). Dans sa description originale, Blainville (1818a: 354$)$ a rattaché à cette espèce deux spécimens figurés par Volta (MNHN.F.BOL136, BOL137 d'une part et MNHN.F.BOL51, BOL52 d'autre part), leur conférant ainsi le statut de syntypes. Blot (1969: 447) a désigné le spécimen MNHN.F.BOL51, BOL52 comme holotype de l'espèce. Cet acte n'est pas valide (ICZN 1999: art. 73.1) ${ }^{\circledR}$ catalogue Bosc n²5 (Fig. 23C, D).

- MNHN.F.BOL58, holotype par monotypie de Spinacanthus cuneiformis (Blainville, 1818) (Volta 1796-1809: pl. 13, fig. 2; Agassiz 1839a: vol. 5, pl. 39, fig. 1; Tyler 1980: 63, fig. 16; Tyler \& Santini 2002) ® catalogue Bosc nº71 (Fig. 23I, J). - MNHN.F.BOL59 et sa contre-empreinte BOL60, lectotype de Proaracana dubia (Blainville, 1818) (Volta 1796-1809: pl. 42, fig. 1; Agassiz 1839a: vol. 2, pl. 74, figs 3, 4; Tyler 1975: pl. 6; Tyler \& Santini 2002). Cette espèce fut créée par Blainville (1818a: 337) sur la base de deux spécimens figurés par Volta (1796-1809: pl. 42, fig. 1 et pl. 65, fig. 2), leur conférant le statut de syntypes. Le spécimen MNHN.F.BOL59, BOL60 ne peut être désigné comme l'holotype contrairement à l'opinion de Tyler (1975:116) et Tyler \& Santini (2002: 83). Le second spécimen figuré par Volta étant semble-t-il perdu, MNHN.F.BOL59, BOL60 est explicitement désigné ici lectotype de Proaracana dubia (Blainville, 1818) ® catalogue Bosc $n^{\circ} 130$ (Fig. 23E, F).

- MNHN.F.BOL65 et sa contre-empreinte BOL66, holotype par monotypie de "Pegasus» volans Volta, 1809 (Volta 1796-1809: pl. 42, fig. 2; Blot 1980: 384). Cette espèce énigmatique est placée avec doute parmi les Lampridiformes (Bannikov 2014; Carnevale et al. 2014) ® catalogue Bosc $\mathrm{n}^{\circ} 128$ (Fig. 19I, J).

- MNHN.F.BOL92 et sa contre-empreinte BOL93, holotype par monotypie d'Eoplatax papilio (Volta, 1809) (Gazola et al. 1794: pl. 5; Volta 1796-1809: pl. 26, fig. 1; Agassiz 1835b: vol. 4, pl. 42 ; Blot 1969: 372, pl. 65-66) ${ }^{\circledR}$ catalogue Bosc n¹31 (Fig. 22C, D).
- MNHN.F.BOL94 et sa contre-empreinte BOL95, syntype de Pycnodus apodus (Volta, 1809) (Volta 1796-1809: pl. 35, fig. 1; Blot \& Voruz 1987: pl. 1-2; Poyato-Ariza \& Wenz 2002: 152) ${ }^{\circledR}$ catalogue Bosc nº 27 (Fig. 19C, D).

- MNHN.F.BOL110 et sa contre-empreinte BOL109, Tylerichthys nuchalis (Agassiz, 1835). Cette espèce fut introduite de manière valide par Agassiz (1835a: 34) en se référant à une figure publiée par Volta (Volta 1796-1809: pl. 22, fig. 1) du spécimen MNHN.F.BOL53, BOL54, qui en est l'holotype par monotypie. Le spécimen MNHN.F.BOL109, BOL110, en revanche, n'a pas été figuré par Volta, ni mentionné par Agassiz ${ }^{\circledR}$ catalogue Bosc n 59 (Fig. 22G, H).

- MNHN.F.BOL120 et sa contre-empreinte BOL121, holotype par monotypie de Callipteryx recticaudus Agassiz, 1839 (Volta 1796-1809: pl. 30 ; Agassiz 1839b: vol. 4, pl. 33, fig. 2). Agassiz avait d'abord rattaché ce spécimen à Callipteryx speciosus Agassiz, 1835, dont il est un des syntypes avec le spécimen MNHN.F.BOL579a, BOL579b et un autre spécimen représenté par Volta (1796-1809: pl. 15) et Bosc (catalogue $n^{\circ} 9$, voir plus loin dans le paragraphe «Autres spécimens remarquables»). Agassiz (1839b: 198) a ensuite fait de MNHN.F.BOL120, BOL121 une espèce distincte ${ }^{\circledR}$ catalogue Bosc n¹5 (Fig. 23A, B).

- MNHN.F.BOL122 et sa contre-empreinte BOL123, holotype par monotypie de Pseudosyngnathus opisthopterus (Agassiz, 1835) (Volta 1796-1809: pl. 58, fig. 1) $®$ catalogue Bosc n66 (Fig. 20C, D).

- MNHN.F.BOL214 et sa contre-empreinte BOL213, Archaephippus asper (Volta, 1809) (Volta 1796-1809: pl. 10, fig. 1; Blot 1969: pl. 61). Ce spécimen faisait partie du cabinet de Vincenzo Bozza (Volta 1796-1809: XLI) avant qu'il ne fût acquis par Gazola ${ }^{\circledR}$ catalogue Bosc nº77 (Fig. 21E, F). - MNHN.F.BOL219, holotype d'Archaephippus asper (Volta, 1809) (Volta 1796-1809: pl. 20, fig. 1; Blot 1969: pl. 60) $\circledR$ catalogue Bosc n¹39 (Fig. 21G, H).

- MNHN.F.BOL234 et sa contre-empreinte BOL233, Ductor vestenae (Volta, 1809) (Volta 1796-1809: pl. 58, fig. 2 ) ${ }^{\circledR}$ catalogue Bosc n⿳68 (Fig. 20K, L).

- MNHN.F.BOL288 [contre-empreinte de BOL285] (Taverne 1998), holotype de Monopteros gigas Volta, 1809 (Volta 17961809: pl. 47) ® catalogue Bosc n6 (Fig. 19E, F).

- MNHN.F.BOL315 et sa contre-empreinte BOL316, Godsilia lanceolata (Agassiz, 1835) ® catalogue Bosc n47 (Fig. 23G, H). - MNHN.F.BOL385 et sa contre-empreinte BOL386, syntype d'Exellia velifer (Volta, 1796) (Volta 1796-1809: pl. 7, fig. 3; Agassiz 1839b: vol. 4, pl. 37, fig. 1 ; Blot 1969: pl. 20). Ce spécimen faisait partie du cabinet de Vincenzo Bozza (Volta 1796-1809: XXVII) avant qu'il ne fût acquis par Gazola.

FIG. 23. - Quelques-uns des spécimens de la «collection Gazola 2 » offerte par Gazola au Premier Consul en 1803 au nom de la ville de Vérone. Cette collection, récupérée à Vérone fin octobre 1805 par Faujas de Saint-Fond, arriva au Muséum national d'Histoire naturelle vers le mois de juin 1806 . Pour chaque spécimen est mis en regard son dessin dans le catalogue de Bosc établi chez Gazola en août 1803 (AN, F/17/1537, sous-dossier Gazola): A, B, Callipteryx recticaudus Agassiz, 1839, holotype; A, MNHN.F.BOL120; B, catalogue Bosc $\mathrm{n}^{\circ} 15$ (1 pied 3 pouces de long $\approx 40 \mathrm{~cm}$ ). C, D, Acanthonemus subaureus (Blainville, 1818), syntype; C, MNHN.F.BOL51; D, catalogue Bosc n²5 (8 pouces de long $\approx 22 \mathrm{~cm}$ ); E, F, Proaracana dubia (Blainville, 1818 ), lectotype; E, MNHN.F.BOL59 (pour respecter le sens du dessin de Bosc, le spécimen est présenté à l'envers, sa face ventrale orientée vers le haut); $\mathbf{F}$, catalogue Bosc $\mathrm{n}^{\circ} 130$ ( 2 pouces de long $\approx$ $5 \mathrm{~cm}$ ); G, H, Godsilia lanceolata (Agassiz, 1835); G, MNHN.F.BOL315; H, catalogue Bosc $n^{\circ} 47$ (9 pouces de long $\approx 24 \mathrm{~cm}$ ); I, J, Spinacanthus cuneiformis (Blainville, 1818), holotype; I, MNHN.F.BOL58; J, catalogue Bosc $n^{\circ} 71(4$ pouces de long $\approx 11 \mathrm{~cm}) ; \mathbf{K}$, L, Eozanclus brevirostris (Agassiz, 1835), holotype; K, MNHN. F.BOL5; L, catalogue Bosc $n^{\circ} 133$ (4 pouces de long $\approx 11 \mathrm{~cm}$ ). Échelles : A, C, G, I, K, $5 \mathrm{~cm}$; E, $3 \mathrm{~cm}$. Crédit (photographies des spécimens originaux) : Recolnat (ANR-11-INBS-0004) - Christian Lemzaouda, 2012. 


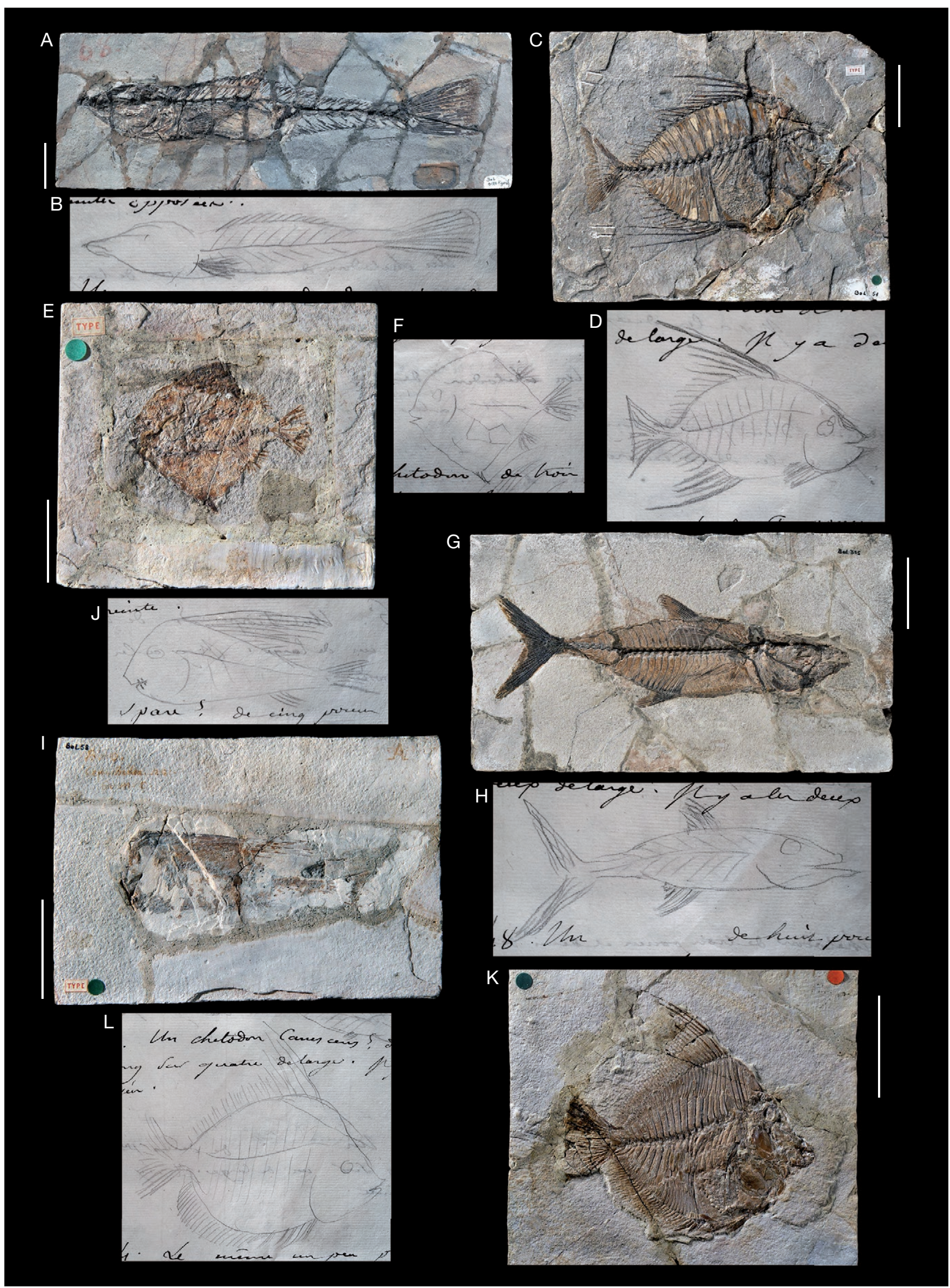


N'étant arrivé à Paris qu'en 1805, la figure publiée par Faujas de Saint-Fond (1803: pl. 5, fig. 2) fut copiée à partir de la planche de l'Ittiolitologia Veronese dont Faujas de Saint-Fond (1803: 130) possédait la première livraison $\AA$ catalogue Bosc $\mathrm{n}^{\circ} 104$ (Fig. 22E, F).

- MNHN.F.BOL407 et sa contre-empreinte BOL408, holotype par monotypie de Paratrachinotus tenuiceps (Agassiz, 1834) (Volta 1796-1809: pl. 39, fig. 3; Agassiz 1834b : vol. 5, pl. 7; Blot 1969: pls 47, 48) ® catalogue Bosc n¹05 (Fig. 20G, H). - MNHN.F.BOL413 et sa contre-empreinte BOL414, holotype par monotypie de Vomeropsis triurus (Volta, 1809) (Volta 1796-1809: pl. 44, fig. 2; Agassiz 1834a: vol. 5, pl. 6; Blot 1969: pls 34, 35) ® catalogue Bosc nº58 (Fig. 20I, J).

- MNHN.F.BOL520 et sa contre-empreinte BOL515, holotype par monotypie de Pygaeus bolcanus (Volta, 1809) (Volta 1796-1809: pl. 59) ® catalogue Bosc n 10 (Fig. 21K, L).

- MNHN.F.BOL533 et sa contre-empreinte BOL532, lectotype de Jimtylerius temnopterus (Agassiz, 1835) (Volta 1796-1809: pl. 45, fig. 2; Bannikov \& Carnevale 2007: fig. 1). Cette espèce fut introduite de manière valide par Agassiz (1835a: 35) en se référant à la figure donnée par Volta (ICZN 1999: art. 12.1 et 12.2 .7$) ~ \circledR$ catalogue Bosc $n^{\circ} 116$ (Fig. 21A, B). - MNHN.F.BOL544a et BOL544b, holotype par monotypie de Xiphopterus falcatus (Volta, 1809) (Volta 1796-1809: pl. 57). Il s'agit probablement du spécimen qui se brisa en deux durant son transport de Vérone à Milan en 1803 (Annexe 1[doc. 47]). Gazola l'avait laissé en dépôt à Milan, où il fut récupéré par Faujas de Saint-Fond en 1806 @ catalogue Bosc n² (Fig. 22K, L).

- MNHN.F.BOL567a et sa contre-empreinte BOL567b, holotype par monotypie de "Platyrhina" gigantea (Blainville, 1818) (Volta 1796-1809: pl. 61; Marramà et al. 2017: figs 12a, 12b) ® catalogue Bosc nº 1 (Fig. 19A, B).

- MNHN.F.BOL576b et sa contre-empreinte BOL576a, holotype par monotypie d'Eoholocentrum macrocephalum (Blainville, 1818) (Volta 1796-1809: pl. 51, fig. 2; Agassiz 1834b: vol. 4, pl. 14, figure du bas; Sorbini \& Tirapelle 1975: pl. 4, fig. 1). Ce spécimen figuré par Volta et dessiné par Bosc appartenait à Gazola. Il est indiqué par erreur dans le catalogue du MNHN établi par J. Blot comme faisant partie de la collection de Drée ${ }^{\circledR}$ catalogue Bosc nº 107 (Fig. 20A, B). - MNHN.F.BOL569b et sa contre-empreinte BOL569a, Sphyraena bolcensis Agassiz, 1835 (Volta 1796-1809: pl. 62). Agassiz (1835a: 36) après avoir d'abord assigné ce spécimen à Sphyraena bolcensis Agassiz, 1835, en fit une espèce séparée, Sphyraena maxima Agassiz, 1835 (Agassiz 1835a: 42), considérée comme synonyme de la première (Woodward 1901: 366) ® catalogue Bosc n³ (Fig. 22A, B).

- MNHN.F.BOL583, triptyque offert au gouvernement français le 20 floréal an 11 (10 mai 1803) alors que Gazola était à Paris (Fig. 13).

\section{AUTRES SPÉCIMENS REMARQUABLES}

Certains spécimens du catalogue établi par Bosc en 1803 à Vérone ne sont pas conservés au MNHN. Il peut s'agir, soit de spécimens perdus, soit de spécimens qui n’avaient pas été choisis par Gazola pour être donnés au MNHN et qui sont conservés aujourd'hui à Vérone, au MCSNV. Sont listés ci-après quelques spécimens figurés par Volta (1796-1809) faisant partie de cet ensemble:

- Perdu, syntype de Callipteryx speciosus Agassiz, 1835 (Volta 1796-1809: pl. 15) ® catalogue Bosc n9 (Fig. 24K, L).

- Perdu, Pygaeus bolcanus (Volta, 1809) (Volta 1796-1809: pl. 46; Agassiz 1843c: vol. 4, pl. 20). Identifié comme Labrus punctatus Linnaeus, 1758 par Volta, le spécimen est un des syntypes de Pygaeus gigas Agassiz, 1835, junior synonyme objectif de Pygaeus bolcanus (Volta, 1809) ${ }^{\circledR}$ catalogue Bosc $\mathrm{n}^{\circ} 14$ (Fig. 24A, B).

- Perdu, holotype par monotypie d' "Engraulis» evolans (Blainville, 1818) (Volta 1796-1809: pl. 22, fig. 2) ® catalogue Bosc $\mathrm{n}^{\circ} 113$ (Fig. 24E, F).

- Perdu, lectotype de Mesogaster sphyraenoides Agassiz, 1835 (Volta 1796-1809: pl. 14, fig. 3; Agassiz 1839a: vol. 5, pl. 38, fig. 3; Bannikov 2008b: 89) ® catalogue Bosc nº 137 (Fig. 24I, J).

- Perdu, holotype de Calamostoma lesiniforme (Volta, 1809) (Volta 1796-1809: pl. 39, fig. 1; Bannikov \& Carnevale 2017) ${ }^{\circledR}$ catalogue Bosc nº141 (Fig. 24C, D).

- Perdu, spécimen attribué à Chaetodon orbis Bloch, 1787 par Volta (1796-1809: pl. 48, fig. 4), identifié avec doute par Agassiz (1835a: 36) comme un jeune Acanthonemus filamentosus Agassiz, 1835, junior synonyme d'Acanthonemus subaureus (Blainville, 1818) (Blot 1969: 446, note de bas de page) $\AA^{\circledR}$ catalogue Bosc $n^{\circ} 147$ (Fig. 24G, H).

- MCSNV VIII.D.112, VIII.D.111 (empreinte et contreempreinte), Archaephippus asper (Volta, 1809) (Volta 17961809: pl. 43) ® catalogue Bosc ${ }^{\circ} 13$.

- MCSNV VIII.C.62, VIII.C.63 (empreinte et contre-empreinte), Scatophagus frontalis (Agassiz, 1835a) (Testa 1793: pl. 3; Volta 1796-1809: pl. 10, fig. 2). Ce spécimen faisait partie du cabinet de Vincenzo Bozza (Volta 1796-1809: XLIV) avant qu'il ne fût acquis par Gazola ${ }^{\circledR}$ catalogue Bosc n ${ }^{\circ} 82$.

Il est à noter que le spécimen MNHN.F.BOL92, BOL93, holotype par monotypie d'Eoplatax papilio (Volta, 1809) a une histoire intéressante (Fig. 22C, D). Il était à Vérone quand le cabinet Gazola fut réquisitionné en 1797 puisqu'il avait été figuré dans un ouvrage publié en 1794 (Gazola 1794: pl. 5). Il fait donc partie des spécimens qui avaient échappé à l'attention des commissaires chargés de rechercher les objets de science et d'art en Italie, probablement parce qu'ils avaient été dissimulés. C'est également le cas du spécimen MCSNV VIII.C.62, 63 qui avait été figuré en 1793 (Testa 1793: pl. 3), mais contrairement au spécimen MNHN.F.BOL92, BOL93 qui fut donné à Faujas de Saint-Fond en 1805, MCSNV VIII.C.62, 63, quant à lui, resta à Vérone.

\section{CONCLUSION}

La question posée en introduction pour essayer de qualifier l'arrivée en France d'une bonne partie de la collection Gazola, n'appelle donc pas une réponse unique. Il convient tout d'abord de distinguer deux collections. La première et la plus importante en nombre (collection Gazola 1) fut 

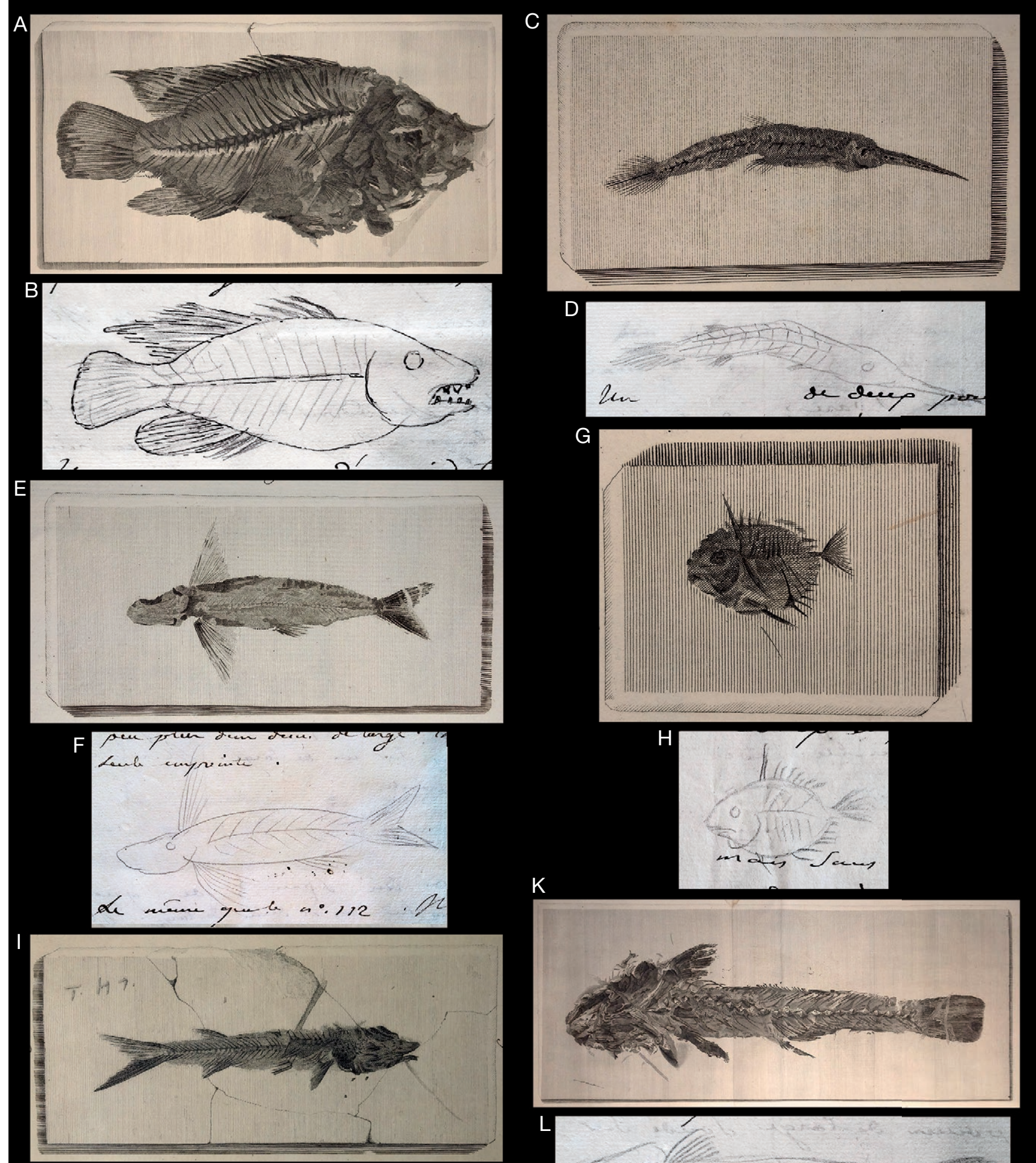

K
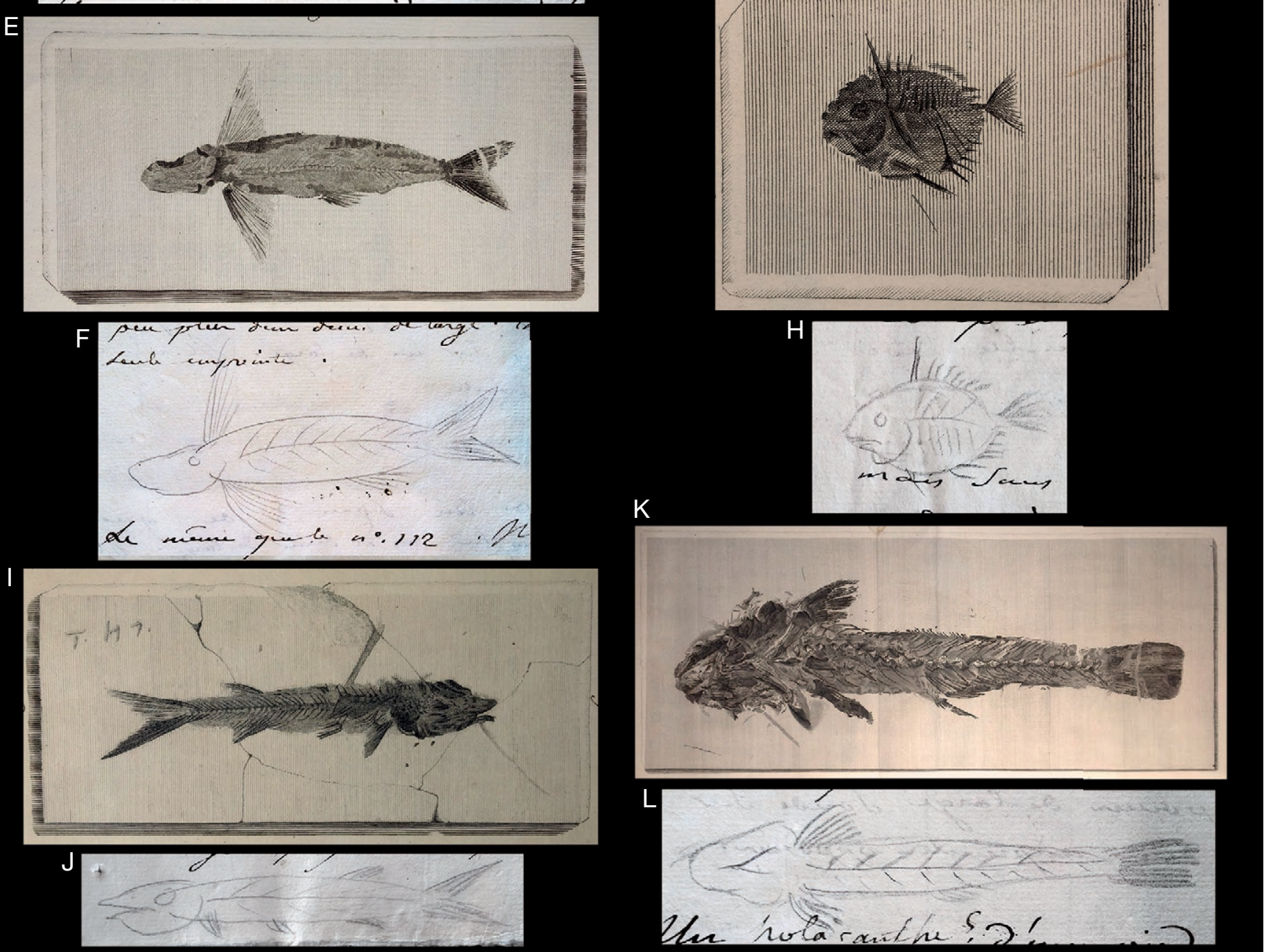

FIG. 24. - Spécimens de la collection Gazola, figurés dans l'Ittiolitologia Veronese et dessinés par Bosc à Vérone en août 1803 (AN, F/17/1537, sous-dossier Gazola), ne faisant pas partie des collections du MNHN: A, B, Pygaeus bolcanus (Volta, 1809); A, figure donnée par Volta (1796-1809: pl. 46); B, catalogue Bosc $\mathrm{n}^{\circ} 14$ (1 pied de long $\approx 32 \mathrm{~cm}$ ); C, D, Calamostoma lesiniforme (Volta, 1809), holotype; C, figure donnée par Volta (1796-1809: pl. 39, fig. 1); D, catalogue Bosc $n^{\circ} 141$ (3 pouces de long $\approx 8 \mathrm{~cm}$ ); E, F, « Engraulis » evolans (Blainville, 1818), holotype; E, figure donnée par Volta (1796-1809: pl. 22, fig. 2); F, catalogue Bosc $n^{\circ} 113$ (4 pouces de long $\approx 11 \mathrm{~cm}$ ); $\mathbf{G}, \mathbf{H}$, jeune individu d'Acanthonemus subaureus (Blainville, 1818)?; G, figure donnée par Volta (1796-1809: pl. 48, fig. 4); H, catalogue Bosc $n^{\circ} 147$ (1 pouce de long $\approx 3 \mathrm{~cm}$ ); I, J, Mesogaster sphyraenoides Agassiz, 1835, lectotype; I, figure donnée par Volta (1796-1809: pl. 14, fig. 3); J, catalogue Bosc $n^{\circ} 137$ (4 pouces de long $\approx 11 \mathrm{~cm}$ ); K, L, Callipteryx speciosus Agassiz, 1835, syntype; K, figure donnée par Volta (1796-1809: pl. 15); L, catalogue Bosc $n^{\circ} 9$ (1 pied 8 pouces de long $\approx 54 \mathrm{~cm}$ ). 
déposée au MNHN en septembre 1798. Cette collection fut d'abord confisquée par les commissaires chargés de rechercher des objets de science et d'art qui accompagnaient l'Armée d'Italie sous les ordres du général en chef Bonaparte. Avec l'accord de ce dernier, Berthollet veilla à ce que Gazola fût indemnisé. Même si Gazola n'eut pas la possibilité de s'y opposer, vu les circonstances, cette réquisition prit donc la forme d'une transaction. Les nombreux rapports, lettres et arrêts qui émanèrent du ministère de l'Intérieur entre 1797 et 1815, au gré des différents régimes politiques, démontrent que l'affaire Gazola ne fut pas prise à la légère et que la volonté de légaliser la situation par des mesures concrètes fut réelle. Il ne faut néanmoins pas occulter que l'encaissement de son indemnisation fut pour le moins chaotique et Gazola dut se montrer pugnace pour faire valoir ses droits. Il obtint ainsi, en plus d'une rente d'environ 2300 francs par an qu'il perçut dès le mois de septembre 1797 , une gratification annuelle de 2500 francs à partir de 1803 . Ces éléments étaient jusqu'alors inconnus par les historiens des sciences. Les professeurs du MNHN, comme Faujas de Saint-Fond, ont parfois ainsi été jugés trop durement en leur prêtant la volonté de travestir la vérité et de faire passer comme une transaction à l'amiable ce que l'on pensait être un "simple" acte de pillage.

Il faut également rappeler que Gazola profita de la mise en place de la République italienne, voulue par Bonaparte, pour occuper un rôle politique de premier plan à Vérone. Gazola ne semble donc pas avoir éprouvé de la rancœur vis-à-vis du gouvernement français puisqu'il offrit, au nom de sa ville, une seconde collection de poissons fossiles en 1803 pour compléter la première. Cette collection (collection Gazola 2) arriva, non sans vicissitudes, à Paris vers le mois de juin 1806. Cet épisode est, quant à lui, sans équivoque: Gazola a bien fait don de cette seconde collection à Napoléon Bonaparte, Premier Consul lorsqu'il l'accepta puis Empereur lorsqu'elle arriva au MNHN. Il y eut bien une tentative du ministre de l'Intérieur de l'époque, Antoine Chaptal, de prendre plus que ce que Gazola voulait offrir. L'intervention de Bonaparte fut déterminante pour défendre Gazola qui eut, dans le cas de cette seconde collection, toute liberté de remettre au Muséum les spécimens de son choix.

\section{Remerciements}

Ce travail a été grandement facilité par la mise en ligne de l'ensemble de la collection des poissons fossiles du Monte Bolca conservée au MNHN (projet national e-recolnat). Les prises de vue et l'informatisation de cette collection ont notamment été réalisées par C. Lemzaouda et E. Porez; Je remercie Elda Frigato (Biblioteca Civica, Verona) pour la communication du portrait de Gazola; Roberto Zorzin (Museo civico di storia naturale di Verona) pour les photographies de spécimens qu'il m’a communiquées; les équipes des Archives nationales, site de Pierrefitte-sur-Seine et des Archives départementales de l'Isère à Grenoble, pour leur accueil. Je remercie enfin les deux rapporteurs de l'article, Giorgio Carnevale et Louis Taverne pour leurs remarques constructives ainsi qu'Emmanuel Côtez pour son travail éditorial.

\section{RÉFÉRENCES}

\section{SOURCES MANUSCRITES}

Archives départementales de l'Isère, Grenoble

J 546 et J 547, Collection Eugène Chaper (1827-1890).

Famille Faujas de Saint-Fond

\section{Archives nationales, Pierrefitte-sur-Seine}

- AF/IV/959, Secrétairerie d'État impériale, feuilles de travail. Sous-dossiers no $298-299$, payement d'une pension au naturaliste Gazola, de Vérone, en échange de ses collections. - AJ/15/596, Minutes des procès-verbaux des assemblées de professeurs du Muséum national d'histoire naturelle et pièces annexes, 1 er semestre 1806.

- F/17/1537, Division des Sciences et Lettres du ministère de l'Instruction publique et des services qui en sont issus. Tome I. Affaires diverses (F-G).

- sous-dossier Faujas [demande de rétablissement de sa pension; compte rendu de ses voyages annuels (an VI-1807)].

- sous-dossier Gazola, de Vérone [demande le payement d'une collection de poissons pétrifiés (an XI-1815)].

- F/17/3979, Muséum d'Histoire naturelle, Collections, sousdossier 13 (1806, Mission de Faujas de Saint-Fond en Italie). - O/3/841, Maison du Roi. Distinctions honorifiques sous la Restauration (1815-1830), sous-dossier 3 [Gazola (comte de). Vérone (Italie). Demande le grade de commandeur de la Légion d'honneur. Rapport au roi approuvé le 12 mai 1815].

\section{Collection privée}

Lettre de Louis Augustin Guillaume Bosc à Giuseppe Angelo Saluzzo di Menusiglio, Milan 27 fructidor an XI

\section{SOURCES IMPRIMÉES}

AgASsiz J.-L.-R. 1833. - Recherches sur les poissons fossiles, lère livraison (juillet 1833). Petitpierre et Prince (texte) et H. Nicolet (planches), Neuchâtel, vol. 1: [i]-xii, [1]-16 (page de titre du volume 1 datée 1833 , texte dans sa première version), pls A-G; vol. 2: [1]-48, pls A-C, 1-7; vol. 4: 17-32, pls A, 1, 2; vol. 5, 1ère partie: 17-24, pls A, 1, 2. https://doi.org/10.5962/bhl.title.4275

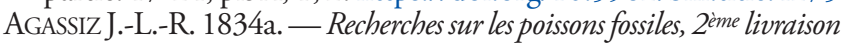
(février 1834). Petitpierre et Prince (texte) et H. Nicolet (planches), Neuchâtel, vol. 1: 17-40 (texte dans sa première version); vol. 2: 49-84, pls F, 11, 12, 15, 16, 22, 26-30, 32, 33, 40; vol. 4: pls 3-6; vol. 5, 1 ère partie: 25-32; pls 3-6; feuilleton additionnel: [1]-20; liste des souscripteurs: [1]-2. https://doi.org/10.5962/bhl.title.4275

AgASSIZ J.-L.-R. 1834b. - Recherches sur les poissons fossiles, 3eme livraison (août 1834). H. Nicolet (planches), Neuchâtel, vol. 2: pls 9, 13, 14, 24, 34; vol. 4: pls B, E, G, 7, 10-12, 14, 21, 22; vol. 5: pls 7, 8, 11-13. https://doi.org/10.5962/bhl.title.4275

AGASSIZ J.-L.-R. 1835a. - Recherches sur les poissons fossiles, $4^{i m e}$ livraison (janvier 1835). Petitpierre et Prince (texte) et H. Nicolet (planches), Neuchâtel, vol. 2: pls 49, 54, 55, 60; vol. 3: pls D, 46; vol. 4: 33-52, pls 23, 38; vol. 5: pls F, 11a, 20, 24, 50, 51, 54-59; feuilleton additionnel: 21-64; liste des souscripteurs: [3]-5. https://doi.org/10.5962/bhl.title.4275

AgASSIZ J.-L.-R. 1835b. - Recherches sur les poissons fossiles, 5eme livraison (juin 1835). Petitpierre et Prince (texte) et $\mathrm{H}$. Nicolet (planches), Neuchâtel, vol. 2: [i]-[iv], 85-200, pls 21, 23, 25c, 30a-30c, 35, 65; vol. 3: pls E-H, 26-29; vol. 4: pls J, 37, 40, 42, 43; vol. 5: pls L, 14, 19, 23, 25, 27, 52; feuilleton additionnel: 65-74. https://doi.org/10.5962/bhl.title.4275 
AgASsiz J.-L.-R. 1836. - Recherches sur les poissons fossiles, Gime livraison (mars 1836). Petitpierre (texte) et H. Nicolet (planches), Neuchâtel, vol. 2: 201-224, pls D, 1c-1e, 2b-2d, 4b, 4c, 8, 10, 10a, 10c, 14b, 14c, 23d, 25, 25a, 25b, 25d, 25e; vol. 4: 53-108; feuilleton additionnel: 75-86. https://doi.org/10.5962/bhl.title.4275

AGASSIZ J.-L.-R. 1837. - Recherches sur les poissons fossiles, gime et gime livraisons (septembre 1837). Petitpierre (texte) et $\mathrm{H}$. Nicolet (planches), Neuchâtel, vol. 2: 225-264 (première version des pages 263-264), pls 1a, 1b, 39 (par erreur 49), 48, 65a bis (par erreur 65a), 65b, 65c, 65d, 66a; vol. 3: [i]-viii, 1-[72], pls B, C, 10a, 10b, 13, 14, 16, 21, 25a, 40a; vol. 4: pls C, D, F, H, K, L, 8, 9 , 11a, 13, 14a-14e; vol. 5: pls D, G, 25a-25c, 60a-60c; feuilleton additionnel: 99-106. https://doi.org/10.5962/bhl.title.4275

AgASSIZ J.-L.-R. 1839a. - Recherches sur les poissons fossiles, $10^{\mathrm{em}}$

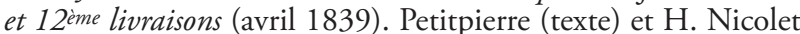
(planches), Neuchâtel, vol. 2: pls 23d (bis) (numéroté 23e par erreur), 33b, 41, 50-53, 53a, 56-58, 58a, 59, 61, 61a, 62-64, 66 (numéroté par erreur 65), 67-69, 69a (numéroté par erreur 60a), 70-72, 72a, 73, 74; vol. 3: 141-156, pls 9, 23, 25, 30a; vol. 4: [i]-xvi, 1-16, 16*-[16**]; vol. 5, 2ème partie: [1]-56, pls 38-42, 49, 53, 60d; feuilleton additionnel: 117-126. https:// doi.org/10.5962/bhl.title.4275

AgASSIZ J.-L.-R. 1839b. - Recherches sur les poissons fossiles, 13 ime livraison (novembre 1839). Petitpierre (texte) et H. Nicolet (planches), Neuchâtel, vol. 2: pl. 69b; vol. 4: 109-204, pls 15-17, 26-36, 37a, 39, 41, 41a, 44; vol. 5: pls 15, 26, 30, 31, 43, 61; feuilleton additionnel: 127-130. https://doi.org/10.5962/bhl.title.4275

AgASSIZ J.-L.-R. 1842. — Recherches sur les poissons fossiles, 14 ème livraison. Jent et Gassmann, Soleure (texte) et H. Nicolet, Neuchâtel (planches), vol. 2: pls 45-47, 51a, 56a, 57a, 57b, 59a, 69c, 75; vol. 4: 205-291, 1-22; vol. 5: pls 16-18, 21, 21a, 22, 32-34, 34a, 35-37, 37a, 62-64; feuilleton additionnel: 131-138. https:// doi.org/10.5962/bhl.title.4275

AgASSIZ J.-L.-R. 1843a. - Recherches sur les poissons fossiles, $15^{\text {eime }}$ et 16 ime livraisons. Jent et Gassmann, Soleure (texte) et $\mathrm{H}$. Nicolet, Neuchâtel (planches), vol. 2, 2ème partie: [1]-72, pls B', B”, Ca, G, 23b, 23c, 31, 33a, 36-38, 42a, 44; vol. 3: 157$390,382^{*}-382^{* *}, 1-32,[33]-[34]$ (fautes à corriger), pls 1,18 22, 22a, 22b, 26a, 38, 40b-40d, 45, 47; vol. 4: pl. 23b; vol. 5, 2ème partie: 57-84, pls B, C, E, H, J, K, 9, 10, 28, 29, 37b (par erreur 37), 44-48; feuilleton additionnel: 139-144. https://doi. org/10.5962/bhl.title.4275

AgASSIZ J.-L.-R. 1843b. - Recherches sur les poissons fossiles, 17 ème livraison. Jent et Gassmann, Soleure (texte) et H. Nicolet, Neuchâtel (planches), vol. 2: [i]-xii, 263-310 (nouvelle version des pages 263-264); vol. 2, 2ème partie: [i]-[ii], 73-336, [337]-[338] (fautes à corriger); pls E, H, 18, 23e, 65a; vol. 3: pls A, J-L,M,M', M", N-Q, 36, 37, 37a; vol. 5: [i]-xii, 1-16; vol. 5, 1ère partie: [16a]-16h, 33-122; vol. 5, 2ème partie: 85-160, pl. M. https:// doi.org/10.5962/bhl.title. 4275

AgASSIZ J.-L.-R. 1843c. - Recherches sur les poissons fossiles, 18 ime livraison. Jent et Gassmann, Soleure (texte) et H. Nicolet, Neuchâtel (planches), vol. 1: [i]-xxxii, 1-188, 1 pl. dans le texte, pls H, J, K; vol. 2: 105-106 (carton), 249-250 (carton), pls J, 17, 19, 23a, 27a, 29b, 29c, 34a, 47a, 55a, 58b, 63a; vol. 3: [i][iv], 13-14 (carton), 207-208 (carton), pls R, S; vol. 4: [i]-[iv], 293-296, 15-16 (carton); pls 19, 20; vol. 5, 1ère partie, 31-32 (carton); vol. 5, 2 ème partie: 3-4 (carton); pls 51a-51c; faux-titres et titres des cinq volumes de l'atlas; ordre des planches pour les cinq volumes de l'atlas; avis aux relieurs: $1 \mathrm{p}$. https://doi. org/10.5962/bhl.title.4275

AuBER J. B. D. (éd.) 1802. - Collection complète des tableaux historiques de la Révolution française, tome 2. Pierre Didot l'aîné, Paris, [i]-[iv] + 273-424, 1 frontispice, pls 69-144. https://doi. org/10.3931/e-rara-49880

BANNIKOV A. F. 2006. - Fishes from the Eocene of Bolca, northern Italy, previously classified in the Sparidae, Serranidae and Haemulidae (Perciformes). Geodiversitas 28 (2): 249-275.
BANNIKOV A. F. 2008a. - Revision of some Eocene fishes from Bolca, Northern Italy, previously classified with the Apogonidae and Enoplosidae (Perciformes). Studi e Ricerche sui Giacimenti terziari di Bolca 12: 65-76.

BANNIKOV A. F. 2008b. - Revision of the atheriniform fish genera Rhamphognathus Agassiz and Mesogaster Agassiz (Teleostei) from the Eocene of Bolca, Northern Italy. Studi e Ricerche sui Giacimenti terziari di Bolca 12: 77-97.

BANNIKOV A. F. 2014. - The systematic composition of the Eocene actinopterygian fish fauna from Monte Bolca, northern Italy, as known to date. Studi e Ricerche sui Giacimenti terziari di Bolca 15: 23-34.

Bannikov A. F. \& Carnevale G. 2007. - The Eocene "Dules" temnopterus Agassiz, 1836 from Monte Bolca, and problem of classifying fossil percoid fishes. Palaeontografia Italica 91: 69-84.

BANNIKOV A. F. \& CARNEVAlE G. 2017. — Eocene ghost pipefishes (Teleostei, Solenostomidae) from Monte Bolca, Italy. Bollettino della Società Paleontologica Italiana 56 (3): 319-331.

Bardet N. \& JaGT J. W. M. 1996. - Mosasaurus hoffmanni, le "Grand Animal fossile des Carrières de Maestricht ": deux siècles d'histoire. Bulletin du Muséum national d'Histoire naturelle, 4ìme série, section $C, 18$ (4): 569-593.

Bertrand G. 2008. - Le Grand Tour revisité. Pour une archéologie du tourisme: le voyage des Français en Italie, milieu XVIIIe siècle début XIXe siècle. École française de Rome, Rome, viii + 791 p.

Blainville H. M. D. DE 1818a. - Poissons fossiles, in Nouveau Dictionnaire d'Histoire Naturelle appliquée aux arts, à l'agriculture, à l'économie rurale et domestique, à la médecine, etc. par une société de naturalistes et d'agriculteurs, tome 27, (Pla-Por). Deterville, Paris: 310-395. https://biodiversitylibrary.org/page/19426101

BlaINVILLE H. M. D. DE 1818b. - Sur les ichthyolites ou les poissons fossiles. Extrait du nouveau Dictionnaire d'Histoire naturelle, Paris, $91 \mathrm{p}$.

BLOCH M. E. 1787. - Naturgeschichte der ausländischen Fische, Dritter Theil. Berlin, [ii] + x + [ii] + 146 p., pls 181-216. https:// biodiversitylibrary.org/page/41327181

BLOT J. 1969. - Les poissons fossiles du Monte Bolca: classés jusqu'ici dans les familles des Carangidae, Menidae, Ephippidae, Scatophagidae. Studi e Ricerche sui Giacimenti terziari di Bolca $1: \mathrm{x}+525 \mathrm{p} ., 100 \mathrm{pls}$

BLOT J. 1980. - La faune ichthyologique des gisements du Monte Bolca (Province de Vérone, Italie). Catalogue systématique présentant l'état actuel des recherches concernant cette faune. Bulletin du Muséum national d'Histoire naturelle, 4ìme série, section C, 2 (4): 339-396.

Bцот J. 1984. - Les apodes fossiles du Monte Bolca, II. Studi e Ricerche sui Giacimenti terziari di Bolca 4: 61-264.

BцOT J. 1987. — L'ordre des Pycnodontiformes. Famille des Palaeobalistidae Nov. Fam. Studi e Ricerche sui Giacimenti terziari di Bolca 5: 87-117.

Blot J. \& Tyler J. C. 1990. - New genera and species of fossil surgeon fishes and their relatives (Acanthuroidea, Teleostei) from the Eocene of Monte Bolca, Italy, with application of the Blot formula to both fossil and recent forms. Studi e Ricerche sui Giacimenti terziari di Bolca 6: 13-92.

Blot J. \& Voruz C. 1975. — La famille des Zanclidae. Studi e Ricerche sui Giacimenti terziari di Bolca 2: 233-277.

Blot J. \& Voruz C. 1987. — L'ordre des Pycnodontiformes. Famille des Pycnodontidae (Agassiz, 1833). Studi e Ricerche sui Giacimenti terziari di Bolca 5: 11-86.

BRIGNON A. 2014. — Les recherches paléoichthyologiques et géologiques sur le gisement permien de Muse près d'Autun (Saône-et-Loire) au début du XIXe siècle. Bulletin de la Société géologique de France 185 (4): 233-252. https://doi.org/10.2113/ gssgfbull.185.4.233

BRIGNON A. 2015. - Faujas de Saint-Fond, Reinwardt, Cuvier et les poissons fossiles du Crétacé de la "Montagne Saint-Pierre" de Maastricht (Pays-Bas). Geodiversitas 37 (1): 59-77. https:// doi.org/10.5252/g2015n1a3 
BRIGNON A. 2017. — Les "ossements pétrifiés» du Jurassique des Vaches Noires dans les cabinets d'histoire naturelle du XVIIIe et du début du XIXe siècles. L'Echo des Falaises 21: 7-35.

CAP P.-A. 1854. - Le Muséum d'Histoire naturelle. L. Curmer, Paris, [vi] $+180+238+[1]$ p., 41 pls. https://gallica.bnf.fr/ ark:/12148/bpt6k63772548

CAPASSO L. 2014. - The history of the fossil fish private collecting. Bollettino del Museo Civico di Storia Naturale di Verona 38 (Geologia Paleontologia Preistoria): 51-89.

CappetTa H. 1975. - Les sélaciens éocènes du Monte Bolca. I. Les Carcharhinidae. Studi e Ricerche sui Giacimenti terziari di Bolca 2: 279-314.

Carnevale G., Bannikov A. F., Marramà G., Tyler J. C. \& ZorZIN R. 2014. - The Pesciara-Monte Postale Fossil-Lagerstätte: 2. Fishes and other vertebrates, in PAPAZZONI C. A., GIUSBERT L., Carnevale G., Roghi G., Bassi D. \& Zorzin R. (éds), The Bolca Fossil-Lagerstätten: A Window into the Eocene World. Excursion guidebook of the conferences CBEP 2014, EPPC 2014, EAVP 2014 and Taphos 2014. Rendiconti della Società Paleontologica Italiana 4: 37-63.

Clément G. 2017. — La collection de poissons fossiles de Monte Bolca du Muséum national d'histoire naturelle. Géochronique 141: 40-43.

DARU P.-B. 1821. - Histoire de la République de Venise, tome 8. Firmin-Didot, Paris, 643 p. https://gallica.bnf.fr/ark:/12148/ bpt6k27849m

DelEuZE J. P. F. 1823. - Histoire et description du Muséum royal d'Histoire naturelle. M. A. Royer, Paris, tome 1: vi + 1-330, 3 plans, 5 pls; tome 2: [iv] + 331-720, 9 pls. https://doi.org/10.5962/ bhl.title.122312

Demougeot A. 1974. - Emmanuel de Haller. Recherches régionales, Centre de Documentation des Archives départementales des Alpes-Maritimes 52: 2-20.

DRYANDER J. 1799. - Catalogus bibliothece historico-naturalis Josephi Banks, vol. 4, mineralogi. Typis Gul. Bulmer, Londres, ix + 390 + [26] p. https://doi.org/10.5962/bhl.title.124897

FALIPOU C. 2017. - Monte Bolca: histoire de la genèse d'un gisement à travers l'évolution de la pensée scientifique. Fossiles, Revue française de Paléontologie 32: 5-20.

FaLIPOU C. 2018. — Les Cerato: 250 ans de fouilles et de passion dans les mines de Bolca (Vénétie, Italie). Fossiles, Revue française de Paléontologie 33: 21-36.

Fara E., Gayet M. \& Taverne L. 2010. - The fossil record of Gonorynchiformes, in GRANDE T. \& POYATO-ARIzA F. J. \& Diogo R. (éds), Gonorynchiformes and Ostariophysan Relationships: A Comprehensive Review. Science Publishers, Enfield: 173-226.

Fauchat N. 1809. - Notice biographique sur S. Exc. Mgr. Emmanuel Crétet, comte de Champmol. Imprimerie Testu, Paris, 16 p.

Faujas de SAinT-Fond B. 1803. — Essai de Géologie, ou Mémoires pour servir à l'histoire naturelle du globe, tome 1. C. F. Patris, Paris, [iv] + 492 p. (1-400 et 403-[494]), 18 pls.

FAUJAS DE SAINT-FOND B. 1804. - Mémoire sur quelques fossiles rares de Vestena Nova dans le Véronais, qui n'ont pas été décrits, et que M. de Gazola a donnés au Muséum national d'histoire naturelle en l'an 11. Annales du Muséum national d'Histoire naturelle 3: 18-24, pl. 1. https://biodiversitylibrary.org/page/14762745

Faujas De SAINT-Fond B. 1806a. - Notice sur le gisement des poissons fossiles et sur les empreintes de plantes d'une des carrières à plâtre des environs d'Aix, département des Bouchesdu-Rhône. Annales du Muséum d'Histoire naturelle 8: 220-226. https://biodiversitylibrary.org/page/14758292

FAUjAS DE SAINT-FOND B. 1806b. - Lettre adressée à M. de Lacépède, sur les poissons du golfe de la Spezzia et de la mer de Gênes. Annales du Muséum d'Histoire naturelle 8: 365-371. https://biodiversitylibrary.org/page/14758455

FAUJAS DE SAINT-FOND B. 1807. - Description géologique des brêches coquillières et osseuses du rocher de Nice, de la montagne de Montalban, de celles de Cimies et de Villefranche, qui tiennent au même système de formation. Annales du Muséum d'Histoire naturelle 10: 409-426. https://biodiversitylibrary.org/ page/3498503

FAUJAS DE SAINT-Fond B. 1808. - Voyage géologique de Nice à Menton, à Vintimille, Port-Maurice, Noli, Savonne, Voltri et Gênes, par la route de la Corniche. Annales du Muséum d'Histoire naturelle 11: 189-225. https://biodiversitylibrary.org/ page/ 28874558

FranÇOIS DE NeufChÂTEAU N. 1798. - Fêtes de la Liberté et entrée triomphale des objets de sciences et d'arts recueillis en Italie. Programme. Imprimerie de la République, Paris, 23 p. https://gallica.bnf.fr/ark:/12148/bpt6k487328

FREYCINET L. DE 1820. - Essai sur la vie, les opinions et les ouvrages de Barthélémy Faujas de Saint-Fond, administrateur du Jardin du Roi, professeur de géologie au Muséum d'Histoire naturelle, membre de diverses sociétés savantes; et chevalier de la légion d'honneur. Imprimerie de Jacques Montal, Valence, 56 p. https://gallica. bnf.fr/ark:/12148/bpt6k110858n

Frigo M. \& Sorbini L. 1997. - 600 fossili per Napoleone. Museo Civico di Storia Naturale di Verona, 31 p.

Gallas L. 1970. - Tendenze illuministiche ed esperienze giacobine a Verona alla fi ne del Settecento. Edizioni "Vita Veronese", Verona, $141 \mathrm{p}$

GaUllieur N.-F. 1856. - Correspondances inédites de Napoléon Ier adressées en Suisse ou à des Suisses. Revue suisse 19: 653-665.

GAUDANT J. 1999. — La querelle des trois abbés (1793-1795) : le débat entre Domenico Testa, Alberto Fortis et Giovanni Serafino Volta sur la signification des poissons pétrifiés du Monte Bolca (Italie). Studi e Ricerche sui Giacimenti terziari di Bolca 8: 159-206.

GAUDANT J. 2005. — Les Pétrifications du Véronois: un manuscrit inachevé de Jean-François Séguier (1703-1784). Studi e Ricerche sui Giacimenti terziari di Bolca 11: 165-290.

GAUDANT J. 2011a. - La publication de l'Ittiolitologia Veronese (1796-1809): le triomphe de l'obstination au service d'une entreprise scientifique hors norme. Studi e Ricerche sui Giacimenti terziari di Bolca 13: 67-133.

GAUDANT J. 2011b. - Brève histoire de la collection Gazola de poissons fossiles éocènes du Monte Bolca (Italie) conservée au Muséum national d'Histoire naturelle, Paris. Geodiversitas 33 (4): 637-647. https://doi.org/10.5252/g2011n4a5

GAZOLA G. B. (DIR.) 1794. — Lettere recentemente pubblicate sui pesci fossili veronesi con annotazioni inedite agli estratti delle medesime. Stamperia Ramanzini, Verona, $187+[1]$ p., 6 pls. https://biodiversitylibrary.org/page/6340899

Girardi M. 1997. — Leonardo Manzati, in MarChI G. P. \& MARINI P. (éds), 1797 Bonaparte in Verona. Museo di Castelvecchio, Verona, 20 settembre 1997-11 gennaio 1998: 249-250.

GiUliari G. C. 1871. — Della tipografia veronese. Saggio storico-letterario. Antonio Merlo, Verona, xiv +196 p.

GuenifFey P. 2011. - Chaptal, un libéral autoritaire. In Histoires de la Révolution et de l'Empire. Éditions Perrin, Paris: 473-519.

Guerra R. 2012. — Don Diego Hurtado di Mendoza, primo collezionista di fossili di Bolca, Studi e Ricerche sui Giacimenti terziari di Bolca 14: 59-83.

GUERRA R. 2014. — I fossili di Bolca nel secolo XVII. Studi e Ricerche sui Giacimenti terziari di Bolca 15: 101-128.

Guerra R. 2015. - Antonio Vallisneri e i fossili di Bolca. Studi e Ricerche sui Giacimenti terziari di Bolca 16: 33-62.

Guerra R. 2016. - Anton Lazzaro Moro e i fossili di Bolca. Studi e Ricerche sui Giacimenti terziari di Bolca 17: 31-61.

Guerra R. 2017. - Francesco Orazio Scortegagna e lo squalo fossile di Bolca. Studi e Ricerche sui Giacimenti terziari di Bolca 18: 15-38.

Il Corriere Milanese 1803. — Parigi 6 fruttidoro (24 agosto). Il Corriere Milanese, 70 (Giovedi 1. Settembre 1803): 565.

INTERNATIONAL COMMISSION ON ZOOLOGICAL NOMENCLATURE (ICZN) 1999. - International Code of Zoological Nomenclature, $4^{\text {th }}$ édition. International Trust for Zoological Nomenclature, London, 306 p. 
Journal De Paris 1803. - Nouvelles de Paris. Journal de Pari 336 (6 fructidor an XI): 2144-2145.

JOURNAL DES DÉBATS 1803. - République française. Journal des débats, et loix du pouvoir législatif, et des actes du gouvernement, 6 fructidor an 11: 2-3.

LACÉPÈDE B.-G. 1798. - Histoire naturelle des poissons, tome 1. Chez Plassan, Paris, [iv] $+8+$ cxlvii +532 p., 25 pls. https://gallica. bnf.fr/ark:/12148/bpt6k97530t

LACOUR P.-Y. 2009. — Les amours de Mars et Flore aux cabinets. Les confiscations naturalistes en Europe Septentrionale 1794-1795. Annales historiques de la Révolution française 4: 73-94. https:// doi.org/10.4000/ahrf.11530

LACOUR P.-Y. 2014. — La République naturaliste. Collections d'histoire naturelle et Révolution française (1789-1804). Muséum national d'Histoire naturelle, Paris, 614 p. (Archives; 19).

LAPORTE M.-A. 2008. - Les saisies d'ceuvres d'art durant le triennio révolutionnaire: réactions françaises et italiennes. Mémoire de Master 1, Université Pierre-Mendès France, $135 \mathrm{p}$.

LIOY P. 1865. - Sopra alcuni avanzi di Plagiostomi fossili del Vicentino e specialmente sull' Aliopsis plejodon Lioy (Galeus cuvieri Ag.). Atti della Società Italiana di Scienze Naturali 8 (4): 398-405, pl. 4.

Marramà G., Carnevale G., Engelbrecht A., Claeson K. M., ZORZIN R., FornASIERO M. \& KRIWET J. 2017. — A synoptic review of the Eocene (Ypresian) cartilaginous fishes (Chondrichthyes: Holocephali, Elasmobranchii) of the Bolca Konservat-Lagerstätte, Italy. Paläontologische Zeitschrift 92 (2): 283-313. https://doi.org/10.1007/s12542-017-0387-z

NiCOLIS E. 1907. - Salone di Paleontologia del Museo Civico di Verona. Madonna Verona 1 (1): 32-49.

PAGÈs F. 1800. - Histoire secrète de la Révolution française, tome 5. J. G. Dentu, Paris, [ii] + 288 p.

Palaiseau J. F. G. 1816. - Métrologie universelle, ancienne et moderne. Lavigne jeune, Bordeaux, xii $+472 \mathrm{p}$.

POMIAN K. 1986. - Collezionisti d'arte e di curiosità naturali, in Storia della cultura veneta, Neri Pozza editore, Vicenza, 5/II: 1-70.

POMMIER É. 1996. — La saisie des oeuvres d'art, in La liberté en Italie (1796-1797), Actes du colloque organisé par le Centre d'études d'histoire de la Défense - 7 juin 1996, Château de Vincennes. https://www.napoleon.org/wp-content/themes/napoleon/annexes/ hors-serie/premiere-campagne-italie/fr/lesecrits/colloques/art. html (consulté le 04/05/2018).

POUCHET F.-A. 1841. - Zoologie classique, ou histoire naturelle $d u$ règne animal, tome $1,2^{e}$ édition. Librairie encyclopédique de Roret, Paris, xvii + 692 p. https://gallica.bnf.fr/ark:/12148/ bpt6k97437889

POYATO-ARIZA F. J. \& Wenz S. 2002. - A new insight into pycnodontiform fishes. Geodiversitas 24 (1): 139-248.

Raux A. 2013. - Poissons fossiles du Monte Bolca (Italie, Eocène). Étude et restauration. Mémoire présenté pour l'obtention du Diplôme national supérieur d'Expression plastique, École supérieure des Beaux-Arts de Tours, Angers, Le Mans, 275 p.

ROCHAS A. 1856. - Biographie du Dauphiné: contenant l'histoire des hommes nés dans cette province, tome 1. Charavay, Paris, xii + $464 \mathrm{p}$.

Roghi G., Dominici S., Giusberti L., Cerato M. \& Zorzin R. 2017. - Historical outline, in Papazzoni C. A., GiUsBerti L., Carnevale G., Roghi G., Bassi D. \& Zorzin R. (éds), The Bolca Fossil-Lagerstätten: $A$ Window into the Eocene World. Excursion guidebook of the conferences CBEP 2014, EPPC 2014, EAVP 2014 and Taphos 2014. Rendiconti della Società Paleontologica Italiana 4: 5-17.

SCORTEGAGNa F. O. 1805. - Descrizione di un pesce pietrificato di singolare grandezza, e spezie esistente in Vicenza presso il r.i. vicario alle miniere. Parise, Vicenza, $22 \mathrm{p}$.
Silvestre A.-F. DE 1829. — Notice biographique sur M. Louis-Augustin-Guillaume Bosc, Membre de l'Institut et de la Société royale et centrale d'Agriculture. Mémoires d'Agriculture, d'Économie rurale et domestique 1829: lxxxi-cvii. https://gallica.bnf.fr/ark:/12148/ bpt6k62899105/f87.item

SORBINI L. 1998. - Le collezioni naturalistiche veronesi nell'1800, in VaCCARi E. (éd.), Le Scienze della Terra nel Veneto dell'Ottocento. Atti del quinto Seminario di Storia delle Scienze e delle Techniche nell'Ottocento veneto, Venezia 20 e 21 ottobre 1995. Istituto Veneto di Scienze, Lettere ed Arti, Venezia: 95-107.

Sorbini L. \& Tirapelle R. 1975. - Gli Holocentridae di Monte Bolca. I: Eoholocentrum, nov. gen., Eoholocentrum macrocephalum (de Blainville) (Pisces-Actinopterygii). Studi e Ricerche sui Giacimenti Terziari di Bolca 2: 205-232.

TAVERNE L. 1998. — Les ostéoglossomorphes marins de l'Eocène du Monte Bolca (Italie) : Monopteros Volta, 1796, Thrissopterus Heckel, 1856 et Foreyichthys Taverne, 1979. Considérations sur la phylogénie des téléostéens Ostéoglossomorphes. Studi e Ricerche sui Giacimenti Terziari di Bolca 7: 67-158.

Temminck C. J. \& Schlegel H. 1843. - Pisces, in Siebold P. F. von (éd.), Fauna Japonica. Leiden, 2-4: 21-72. https://doi. org/10.5962/bhl.title. 124951

TESTA D. 1793. - Breve transunto della lettera terza sui pesci fossili del Monte Bolca. Opuscoli Scelti sulle Scienze e sulle Arti 16: 416-417, pl. 3 .

THOUVENEL P. 1797-1798. - Traité sur le climat de l'Italie considéré sous ses raports phisiques météorologiques et médicaux. Giuliari, Vérone, vol. 1: [iv] + 312 p., 1 portrait; vol. 2: [iv] + 312 p.; vol. 3: [iv] + 312 p.; vol. 4: [iv] + 312 p.

Thouvenel P. 1802. - La guerra di dieci anni raccolta polemico-fisica sull' elettrometria galvano-organica. Verone, $344 \mathrm{p}$.

Tuilier A. 1989. - L'Université de Paris, la Sorbonne et la Révoluion. Catalogue de l'exposition "Célébration du bicentenaire de la Révolution française en Sorbonne», 20 juin-14 juillet 1989. Sorbonne, Paris, $170+[6] \mathrm{p}$.

TYLER J. C. 1975. - A new species of boxfish from the Eocene of Monte Bolca, Italy, the first unquestionable fossil record of the Ostraciontidae. Studi e Ricerche sui Giacimenti terziari di Bolca 2: 103-126.

TYLER J. C. 1980. — Osteology, phylogeny, and higher classification of the fishes of the order Plectognathi (Tetraodontiformes). National Oceanic and Atmospheric Administration, Technical Report, National Marine Fisheries Service, Circular 434: i-xi + 1-422. https://doi.org/10.5962/bhl.title.63022

Tyler J. C. \& SANTini F. 2002. - Review and reconstructions of the tetraodontiform fishes from the Eocene of Monte Bolca, Italy, with comments on related tertiary taxa. Studi e Ricerche sui Giacimenti terziari di Bolca 9: 47-119.

VolTA G. S. 1796-1809. - Ittiolitologia Veronese del Museo Bozziano ora anneso a quello del Conte Giovambattista Gazola e di altri gabinetti di fossili Veronesi. Stamperia Giuliari, Vérone, 1 ère partie: LII p.; $2^{\text {ème }}$ partie: CCCXXIII p., 76 pls. [seules la première partie du texte, les 52 premières pages de la seconde partie et les 11 premières planches parurent en 1796].

WOODWard A. S. 1901. - Catalogue of the Fossil Fishes in the British Museum, Part IV. British Museum (Natural History), London, xxxix + 636 p., 19 pls. https://doi.org/10.5962/bhl. title.61854

ZaGHi C. 1961. - I carteggi di Francesco Melzi d'Eril duca di Lodi. La Vice-Presidenza della Repubblica Italiana, vol. 5 (dal giugno 1803 al 27 gennaio 1804). Museo del Risorgimento e Raccolte storiche del Comune di Milano, Milano, [iv] + 462 + [1] p., 1 frontispice. 


\section{ANNEXES}

ANNEXE 1. - Transcription des documents manuscrits.

\section{DOCUMENT 1 - 20 JUILLET 1797}

Rapport de Rochejean et Boulanger, agents de l'administration des contributions et finances à Vérone, envoyé à Emmanuel de Haller, administrateur des contributions et finances d'Italie (AN, F/17/1537, sous-dossier Gazola).

Verone le 2 Thermidor an 5eme de la Rép. Frane [République française] une, et indivisible.

Le préposés [sic] aux contributions à Verone au Citn [Citoyen] Haller administrateur des contributions et finances d'Italie

Citn administrateur

Nous avons pris des renseignement [sic] sur la prairie que le Général en Chef a accordée en indemnité au Citn Gazzola sur la demande du Citn Bertholet [sic]. Cette prairie est située à Villafranca dans le Veronais; elle sappelle Prebiano; elle est un communal de Villafranca, et d'une seule pièce. (sur les 1075 champs qu'elle contient 40 sont en chemins, canaux, marais of c) le foin s'en afferme par lots au temps de la récolte pour 12000lt [livres] de Venise, déduction faite des frais d'entretien; il est à présumer qu'elle rend d'avantage, la municipalité ayant paru vouloir en atténuer la valeur; Verone avoit cedée [sic] cette prairie à Villafranca avec obligation d'entretenir le château et le mur qui sont à présent detruits; il est constant que l'ex gouvernement venitien avait le droit de disposer des fonds communaux

Pour copie conforme Signé Rochejean, Boulanger

Les agens [sic] de l'administration des contributions, et finances

\section{DOCUMENT 2 - 13 AOÛT 1797}

Arrêté d'Emmanuel de Haller, approuvé par le général Bonaparte (AN, F/17/1537, sous-dossier Gazola) (Fig. 5).

Milan le 26 Thermidor an $5^{\text {eme }}$ de la Repube Françe une, et indivisible.

Haller

Administrateur des contributions et finances d'Italie

Vu la demande faite par le commissaire du gouvernement français à la recherche des objets de sciences et arts en Italie, à l'effet d'obtenir une indemnité en faveur du Citen In Baptiste Gazzola de Verone pour la collection complette de son cabinet d'histoire naturelle composée des poissons plantes pétrifiées [sic] qu'il a cédé à la Republique française, pour augmenter les collections du Museum national d'histoire naturelle de Paris.

L'etat produit par le dit Gazzola des depenses que cette collection lui a couté depuis quinze ans, et les prix qu'il a payé [sic] pour réunir diverses collections à la sienne; ainsi que les rentes viagères d'environ 180 sequins, qu'il paye encore à divers amateurs dont les cabinets lui ont été cédés.
Considerant que la prairie nommée Prebiano près de Villafranca a été cedée par le senat de Venise à cette commune pour fournir aux depenses de l'entretien du château et des murs de Villafranca, lesquelles dépenses n'existent plus.

Arrête

$1^{\circ}$. Que le dit prés [sic] nommé Prebiano est donné et non concedé par la Republique Française en pleine propriété au dit Jn Bte Gazzola de Veronne [sic] pour en disposer par lui comme bon lui semblera.

$2^{\circ}$. Que le Citn Gazzola payera sur le produit dudit prés toutes les années quatre cents livres de France ${ }^{[1]}$ de rente pour un capital de dix mille livres accordés par le Général en Chef à la Société Italienne de Veronne.

$3^{\circ}$ que le dit Gazzola payera de plus conformément aux ordres du Général en Chef, quatre mille livres de France, au Citn Cagnoli [2] astronome de Verone, pour endemnité [sic] qui lui ait due et qui lui a été allonée

Le Gouverment central du Veronnais reste chargé de faire mettre à execution le présent arrêté, et specialement de faire mettre le dit Gazzola en possession sur le champ du dit prés; de manière qu'il ne puisse dans aucun cas, et sous aucun prétexte être troublé dans sa jouissance.

Haller

\section{Approuvé \\ Bonaparte}

\section{DOCUMENT 3 - OCTOBRE 1802}

Rapport de Ferdinando Marescalchi, ministre des Relations extérieures de la République italienne à Napoléon Bonaparte, Président de la République italienne (AN, F/17/1537, sousdossier Gazola).

Le Ministre des Relations extérieures de la République française[3] me transmit, ily a quelque tems [sic] une pétition qui lui avait été adressée par le Cen Gazola de Vérone, lequel réclamait un surplus d'indemnité qu'il prétendait lui être dû pour raison d'une collection de poissons pétrifiés qu'il avait cédée au Gouvernement français.

Le Ministre ajoûta [sic] que cette demande lui avait paru peu fondée et que, s'il m'en donnait connaissance, c'était uniquement pour me mettre à portée d'apprécier les réclamations ultérieures qui pourraient être formées sur ce sujet.

Je pensais aussi que je n'aurais pas à m'occuper davantage de cette affaire. Mais le Cen Gazola a réitéré ses instances; il m'a fait parvenir une nouvelle pétition adressée au Premier Consul; il m'a

${ }^{1}$ La livre de France équivalait à peu près au franc qui l'avait remplacée en 1795 .

2 Antonio Cagnoli (29 septembre 1743, Zante-6 août 1816, Vérone).

3 Charles-Maurice de Talleyrand Périgord (2 février 1754, Paris-17 mai 1838, Paris). 
fait presser de la lui soumettre. Je crois donc devoir la présenter et en rendre compte.

Selon le Cen Gazola, il avait été arrêté que, pour prix de sa collection, on lui donnerait un fonds produisant 6000 francs de rente. Ce fonds avait même été spécialement désigné. C'était la prairie dite de Probiano [Prebiano], située à Ville-franche [Villafranca] dans le Véronais.

Cependant la cession de ces biens rencontra des obstacles. On lui en remit donc un autre dans le Mantouan. Mais celui-ci ne donne pour tout revenu que 3000 livres de Milan. Il faut conséquemment, pour le satisfaire en entier, qu'on lui accorde un surplus de 3750 francs de rente.

Suivant, au contraire, ce que dit le Ministre des Relations extérieures, il y eut, à la vérité, un premier arrêté portant que la prairie de Probiano serait transportée au Cen Gazola. Mais à ces arrêtés succéda un nouvel arrangement qui en annulla [sic] les dispositions. Il n'est donc plus en droit de s'en prévaloir et, à cet égard, tout a été terminé, consommé par les derniers accords par leur exécution.

Maintenant, si l'on consulte les pièces, voici ce qui en résulte.

Il ne paraît pas que, lorsque le Cen Gazola céda sa collection de poissons et de plantes pétrifiées [sic], l'on eut fait avec lui quelque convention.

On ne voit pas non plus qu'il ait été précisément question de lui attribuer 6000 francs de rente: mais ce qui est constant, c'est qu'il fut pourvu à son indemnité par un arrêté du 26 Thermidor an 5 que pris le Cen Haller, que le Général en chef approuva, et dont voici les principales dispositions.

$1^{\circ}$. Il annonce une demande faite, en faveur du Cen Gazola, par la commission du gouvernement français chargée de la recherche des objets de sciences et arts en Italie.

$2^{\circ}$. Il énonce encore un état fourni par le Cen Gazola des dépenses qu'il avait faites pour sa collection, et notamment de diverses rentes viagères s'élevant à 180 sequins qu'il s'était obligé de payer à des amateurs pour prix des cabinets qu'ils lui avaient cédés.

$3^{\circ}$. Il détermine que la prairie de Probiano sera transportée au Cen Gazola en toute propriété.

$4^{\circ}$. Il le charge, moyennant ce transport, de payer une rente perpétuelle de 400lt [livres de France] au capital de 10,000lt accordée à la Société Italienne de Vérone, et de plus une somme de 4,000lt au Cen Cagnoli [1], astronome.

On trouve, du reste, une autre pièce dont il résulte que le Cen Haller, avant de prendre son arrêté, avait voulu se procurer quelques renseignements sur la prairie dont il s'agissait. C'est une lettre qui lui fut écrite le 2 Thermidor an 5 par les Cens Rochejean et Boulanger, préposés aux contributions à Vérone, et dans laquelle ils disent que cette prairie, dont le foin s'affermait annuellement pour 12,000lt de Venise, devait même produire davantage.

C'est là sans doute ce qui a donné lieu au Cen Gazola de dire qu'il avait été décidé de lui adjuger 6000 francs de rente; car c'est à-peu-près à cette somme que reviennent les 12,000 livres de Venise.

Quoiqu'il en soit, cet arrêté demeura sans exécution et, selon les informations qui m'ont été données, la raison en fut dans l'opposition de la commune de Villafranca, à qui cette prairie avait été concédée par l'ancien Gouvernement de Venise et qui était jalouse de conserver une telle propriété.

Il fallut donc pourvoir de quelque autre manière à l'indemnité du Cen Gazola, et c'est ce qu'on fit dans une transaction passée le 8 vendémiaire an 6 entre la République française et l'administration de l'Etat de Mantouan. Il fut dit dans l'art. 3 de cet acte : "ladite administration séparera sur les biens qui lui sont cédés une portion quelconque produisant 3000 livres de Milan, pour être remise en toute propriété au Cen Gazola, à l'effet de l'indemniser du cabinet de poissons pétrifiés et autres objets qu'il a cédé à la République française».

On ne voit point d'ailleurs comment et pour quels motifs on réduisit à ces 3000lt [livres] de Milan ce que l'on avait d'abord pensé d'accorder au Cen Gazola. On ne trouve dans les pièces que la simple copie de cet art. 3, dont les propres termes viennent d'être rapportés. De plus, y a-t-il une acceptation formelle de la part du Cen Gazola, et surtout une acceptation telle qu'elle emporte une renonciation à toute plus ample prétention? C'est ce qu'on ne voit pas davantage.

Tout ce qu'il semble que l'on peut dire, c'est que jamais il n'a été précisément question de traiter avec le Cen Gazola pour le prix de sa collection, et qu'il fait seulement entendre que le Gouvernement français le dédommagerait d'une manière convenable.

Que d'abord on voulut le traiter généreusement et lui accorder non pas seulement une indemnité, mais encore une récompense.

Enfin que, pour des raisons que j'ignore, on revint un peu de ces premières dispositions, et qu'on crut pouvoir le satisfaire en lui donnant à-peu-près la moitié de ce qui lui avait été d'abord décerné.

A présent donc, le Cen Gazola est-il en droit de se plaindre qu'on ne lui a point assez donné et de réclamer un supplément? C'est une question qui me paraît dépendre du prix que l'on peut attacher à sa collection; car, du reste, il ne semble pas qu'il puisse rigoureusement se prévaloir du premier arrêté et en induire un droit formel.

Mais ce prix ne m'est nullement connu et tout ce que j'en puis dire, c'est que le Cen Berthollet, qui s'est vivement intéressé pour le Cen Gazola et a écrit en sa faveur, paraît estimer beaucoup la collection et penser même qu'elle n'était pas trop payée par $6000 f$ de rente.

Du reste, l'affaire regardant spécialement le Gouvernement français, il ne m'appartient point de proposer une décision, et je crois devoir me borner à faire connaître au Premier Consul la réclamation du Cen Gazola.

Paris Octobre 1802.

$n^{a}$. [nota] La lettre du Cen Berthollet et celle du Cen Talleyrand furent remises avec le Rapport au Premier Consul. 


\section{DOCUMENT 4 - SANS DATE [FIN 1802 (APRÈS OCTOBRE) - DÉBUT 1803]}

Lettre de Gazola à Napoléon Bonaparte, Premier Consul (AN, F/17/1537, sous-dossier Gazola).

\section{Au Premier Consul et Président Citoyen Consul,}

Le Citen Gazola de Véronne [sic], a eu l'honneur d'adresser au Premier Consul et Président, une pétition qui lui a été présentée, avec un rapport [Annexe 1(doc. 3)] dans le courant du mois d'octobre dernier, par le Ministre des relations extérieures de la République italienne.

L'objet de sa demande, était le complément du payement d'une collection de poissons, et de plantes pétrifiées, qu'il a cédée en l'an 5 au Gouvernement Français.

Un arrêté pris, par le Cen Haller, le 16 Thermidor [4] de la même année, et approuvé par le Premier Consul lui-même, alors Général en Chef; avait assigné en payement au Cen Gazola, une prairie, située dans la commune de Villafranca, produisant audelà [sic] de six mille francs de rente.

Cette assignation n'ayant pu être effectuée, à cause de certaines difficultés, on dut la remplacer par une autre, et pour lors, au lieu de lui attribuer quelque fonds équivalent, on lui en donna un qui ne vallait [sic] pas même la moitié, puisquion ne lui a fait qu'une assignation de trois mille livres de Milan ou environ 2.322lt de France.

Le Cen Gazola, ne crut point aussi devoir se regarder comme suffisamment indemnisé, soit en égard à la valleur [sic] de sa collection, et aux dépenses qu'elle lui avait occasionnées, soit par rapport aux intentions, que le Premier Consul avait manifestées, par son approbation mise au bas de l'arrêté du 16 Messidor [3] an 5e.

Il a donc osé réclamer un supplément de quatre vingt huit mille francs, savoir $64.000 f$ en capital pour rendre la seconde assignation à peu près égale, à la première, et 24.000f [5] pour les arrérages de six ans.

Mais en se permettant cette fixation, il n'a eu d'autre vue, que celle de présenter, une évaluation quelconque; car du reste il s'en rapporte avec la soumission la plus entière, à la Justice et aux bontés du Premier Consul, quil supplie de vouloir bien prononcer.

Salut Respect et Vénération $J^{n}$ [Jean] Baptiste Gazola

\section{DOCUMENT 5-29 JANVIER 1803}

Lettre de Gazola à Napoléon Bonaparte, Premier Consul, suivie d'une note de la main de Bernard-Germain Lacépède (AN, F/17/1537, sous-dossier Gazola).

4 C'est une erreur, l'arrêté fut pris le 26 Thermidor an 5.

5 Gazola se trompe en sa faveur : 6000 francs -2322 francs = 3678 francs de manque à gagner annuel, soit 22068 francs d'arrérages sur six ans, et non 24000 francs.
Au Premier Consul

Citoyen Premier Consul et Président

Gazola de Vérone, céda, lorsque vous remportiez tant de victoire en Italie, une riche et rare collection de poissons et autres objets pétrifiés, qui forment à présent un des monuments le plus précieux de la Nature.

Je n'ai cessé de m'occuper depuis lors, de nouvelles recherches, j’ai fait fouiller dans la montagne de Vestena Nova, et je n'ai rien épargné pour augmenter au moins du double la première collection.

Je l'ai offerte à ma Patrie, elle vous en fait hommage par mon organe, et elle est à vous dans ce moment, citoyen Consul, si vous daignez l'accepter.

Il serait à propos de faire constater le nombre et la rareté des objets par un Inventaire; assurer leur conservation par la manière de les emballer et de la faire arriver; Le Professeur du Muséum chargé de cette partie, pourrait recevoir vos ordres de se rendre avec moi à Veronne [sic] pour soccuper de cet objet et faire jouir les sciences, d'une collection, qui ne peut qu'augmenter par sa rareté le nombre des Etrangers en France.

Salut Respect et Vénération

\section{Paris ce 9 Pluviose an $11^{e}$}

Jean Baptiste Gazola

Note de Lacépède :

Les naturalistes partagent la reconnaissance du savant et zélé Citoyen Gazola. Sa précieuse collection ne peut pas arriver en France par des soins plus éclairés que ceux du professeur du muséum d'histoire naturelle (le citoyen Faujas) que la loi a chargé de voyager chaque année pour l'accroissement des richesses de ce muséum.

J'ai l'honneur de présenter au Premier Consul l'hommage de mon respect

Signé Lacepède

\section{DOCUMENT 6-FÉVRIER 1803}

Copie d'un rapport de Jean-Antoine Chaptal, ministre de l'Intérieur, au Premier Consul (AN, F/17/1537, sous-dossier Gazola).

Rap ${ }^{r t}$ [rapport] au Premier Consul

Le premier consul et président m'a fait l'honneur de me renvoyer deux pétitions du citn Gazzola, avec ordre de lui en faire un prompt rapport.

Par la première, le citn Gazzola demande d'être entièrement payé du prix d'une collection de poissons pétrifiés qu'il céda an l'an 5, à la République française et pour [...] laquelle il n'a été qu'en partie satisfait.

Cette demande a déjà été transmise au premier consul. J'eus l'honneur moi-même de la lui présenter dans le courant du mois d'octobre dernier avec un rapport qu'il trouva bien de retenir.

Comme aujourd'hui, je ne pourrais il me semble, lui rien dire de plus sur cet objet, je crois devoir me borner à remettre ce même rapport sous ses yeux et en conséquence je prends la liberté d'en joindre ici la copie. 
J'ajồterai [sic] seulement que si le Premier Consul juge que, comme il me l'a paru, la question dépend surtout du prix qu'on peut attacher à la collection dont il s'agit, personne ne serait plus à portée de l'éclairer, que les administrateurs du Jardin des Plantes.

Quant à la seconde pétition, elle a pour objet d'offrir au Premier Consul une nouvelle collection que le Citen Gazzola a formée dans la ville de Vérone, à qui il l'a gratuitement cédée, [et] fait hommage par son organe.

Je n'ai point sans doute à examiner si cette offre doit être acceptée : ce n'est qu'à la sagesse du premier consul qu'il appartient d'en décider.

Je prendrai seulement la liberté d'observer que cette collection jointe à celle qui se trouve déjà dans le Museum du Jardin des Plantes formerait un tout bien précieux et peut-être unique dans le monde.

D'ailleurs si la ville de Vérone veut remplacer dans la suite ce qu'elle aura cédé, la chose ne sera pas infiniment difficile, car la montagne de Vestena nova, d'où l'on tire ces poissons petrifiés en est, pour ainsi dire, une mine inépuisable.

Pour ce qui est [...] de la proposition d'envoyer un des professeurs du Museum à Vérone pour choisir les objets, en faire inventaire, veiller à l'emballage et au transport, cette demande paraît d'autant plus à propos, qu'il est de fait, que la première collection amenée à Paris a beaucoup souffert faute d'avoir pris les précautions nécessaires.

Il semble enfin qu'on ne peut faire de meilleur choix pour cette mission, que celui du citn Faujas indiqué par le citn La Cepède

Paris février 1803

\section{DOCUMENT 7 - FÉVRIER OU MARS 1803}

Rapport du ministère de l'intérieur, au gouvernement de la République (AN, F/17/1537, sous-dossier Gazola).

Proposition d'ordonnance que la créance du Cen Gazola sera acquittée en rescriptions admissibles en payement de domaines nationaux imputables sur le fonds de réserve de dix millions

\section{Le [...] Ventose an 11 de la République}

Rapport au Gouvernement de la République

Le Cen Gazola de Vérone, qui en l'an 5, céda à la République française une suite de poissons et de plantes pétrifiés, sollicite ce qui lui reste dî̀ sur la rente perpétuelle de 6000lt [livres de France] ${ }^{[1]}$ qu'on lui assigna pour cette collection. Il appuie sa demande sur un arrêté pris par le Cen Haller, le 26 thermidor an 5, et que le Premier Consul approuva comme général en chef de l'armée d'Italie.

Cet arrêté ordonnait que le Cen Gazola serait mis sur le champ en possession de la Prairie dite de Probiano [Prebiano] située à Villafranca dans le Véronais, et dont le produit est effectivement de $6000 f$ par an. Mais la cession qui devait lui en être faite, ayant rencontré de grands obstacles, on lui remit un autre bien, situé dans le Mantouan, qui ne donne pour tout revenu que 3,000lt [livres] de Milan. En sorte que pour le satisfaire en entier, il faudrait lui payer une rente de 3,750lt. Il en évalue le capital à la somme de 64,000lt, parceque dans la concession qui lui avait été faite du premier bien national, il était tenu de payer à la Société Italienne de Vérone une rente de $400^{\text {lt }}$ au principal de 10,000lt et d'acquitter une somme de 4,000lt au Citn Cagnoli [2], astronome de Vérone.

Il faut ajouter à ce capital de 64,000lt les arrérages des 3,750lt qui sont dus depuis six ans, montant à la somme de ............ 22,500

c'est par erreur que le total des arrérages est porté dans la pétition à 24,000lt

Total de la somme due au Cen Gazola à $86,500^{l t}$

Maintenant, si on considère de quel intérêt cette collection est pour l'histoire naturelle, ce qu'elle a couté au Cen Gazola pour l'augmenter de toutes celles du même genre qui existaient à Vérone, on ne trouvera pas que la somme demandée soit exorbitante. Au surplus, c'est le sentiment du Sénateur Berthollet; il pense qu'elle n'est pas trop payée par $6000 f$ de rente.

Pour effectuer l'entier payement de ce qui reste dî sur l'achat de cette collection, j'ai l'honneur, Citoyens Consuls, de vous proposer l'arrêté ci-joint, par lequel la créance du Cen Gazola serait acquittée en rescriptions admissibles en payement de domaines nationaux imputables sur le fonds de réserve de 10 millions.

Salut et Respect

\section{DOCUMENT 8 - FÉVRIER OU MARS 1803}

Extrait des registres des délibérations du gouvernement de la République. Un second document intitulé «Minute d'arrêté» contient les mêmes informations (AN, F/17/1537, sousdossier Gazola).

\section{Le [...] Ventose an 11 de la République.}

Le Gouvernement de la République sur le rapport du ministre de l'intérieur arrête

$$
\text { Art. 1er }
$$

La somme de quatre ving six mille cinq cent francs, qui reste due au Cen Gazola sur la vente qu'il a faite au gouvernement français d'une collection de plantes et de poissons pétrifiés, sera acquittée en rescriptions admissibles en payement de domaine national, imputable sur le fonds de réserve de dix millions.

$$
\text { Art. } 2^{e}
$$

Le Ministre des finances est chargé de l'exécution du présent arrêté. Le Premier Consul | Signé Bonaparte Pour le Premier Consul | Le Secrétaire d'Etat 


\section{DOCUMENT 9 - FÉVRIER OU MARS 1803}

Lettre de Jean-Antoine Chaptal, ministre de l'Intérieur, à Gazola (AN, F/17/1537, sous-dossier Gazola).

3e Division | Bureau des sciences | Réponse à la lettre | Enregistrement à l'arrivée $n^{\circ} 4728$

Il est prévenu quil sera pris des mesures pour liquider sa créance

Paris, le ventose an 11 de la République française Le Ministre de l'Intérieur,

Au Citoyen Gazola, hôtel de Versailles, rue Batave

Le premier Consul ma renvoyé, Citoyen, la pétition par laquelle vous sollicitez le payement de ce qui reste dî sur le prix d'une collection de poissons pétrifiés, que vous avez cédée en l'an 5 au Gouvernement français.

Je vous préviens qu’il sera pris les mesures nécessaires pour liquider cette créance.

Quant à la nouvelle collection de poissons pétrifiés, dont vous êtes chargé par la ville de Vérone, de faire hommage au Premier Consul, je m'empresserai de vous faire connaître les intentions du Gouvernement à cet égard; aussitôt qu'il me les aura communiquées.

$$
\text { Je vous salue }
$$

\section{DOCUMENT 10 - SANS DATE (DÉBUT 1803)}

Brouillon d'un rapport du ministre de l'Intérieur destiné à Napoléon Bonaparte, Premier Consul (AN, F/17/1537, sous-dossier Gazola).

Proposition d'accepter l'hommage fait par la ville de Vérone, d'une collection de poissons pétrifiés.

$$
\text { Rapport }
$$

Citn ler Consul

Le Citn Gazola, de Vérone, a offert à sa Patrie une collection de poissons pétrifiés plus nombreuses et plus intéressante que celle quill céda en l'an 5 à la République Françoise.

La ville de Vérone, connaissant tout le mérite de cette nouvelle collection, vous en fait hommage Citn $1^{\text {er }}$ Consul, par l'organe du Citn Gazola. Elle desire vivement que vous daigniez accepter cet [sic] offre.

Dans ce cas, il serait à propos de faire constater le nombre et la rareté des objets; d'en assurer la conservation par la manière de les emballer et de les faires arriver. Le Citn Gazola offre d'accompagner le Professeur d'Hist: Nat: à qui ce soin serait confié, et le Citn Lacépede désigne le Citn Fanjas, que la loi a chargé de voyager chaque année pour l'accroissement des richesses du Museum.

En acceptant cet hommage, Citn ler Consul, vous n'aurez pas à regretter de voir la ville de Vérone privée, sans ressources, de ces objets précieux, puisquil lui sera facile de les remplacer par les nouvelles recherches qu'elle peut ordonner de faire dans la montagne de Vestena Nova, mine vraiment inépuisable de ce genre de curiosité.

En conséquence j'ai l'honneur de vous présenter le projet d'arrêté ci-joint par lequel l'offre faite par la ville de Verone est acceptée.

\section{Arrêté}

tes Consuts Le Gouvnt de la République sur le rapport du Mtre de l'Intérieur, arrêtent [sic] ce qui suit:

Art. 1

E'homage L'offre d'une collection de poissons et autres objets pétrifiés faite au ler Consul par la ville de Verone, est acceptée.

2.

Le Citn Faujas, Professeur-admr [administrateur] du Museum d'hist: nat: se rendra à Vérone pour y constater le nombre et la rareté des objets; en assurer la conservation, et en faire effectuer le transport à Paris.

3.

Le Mrre de l'Intérieur est chargé de l'exécution du présent arrêté.

\section{DOCUMENT $11-1^{\text {ER AVRIL } 1803}$}

Extrait des registres des délibérations du gouvernement de la République. Un second document intitulé "Minute d'arrêté" contient les mêmes informations (AN, F/17/1537, sousdossier Gazola).

\section{le 11 Germinal an 11 de la République.}

Le Gouvernement de la République sur le rapport du ministre de l'intérieur arrête

Art. Ler

L'offre d'une collection de poissons et autres objets pétrifiés, faite au Premier Consul par la ville de Vérone est acceptée.

Art. $2^{e}$

te Cit. Fanjas, Professeur administrateur dumuséum d'histoire naturette se rendra à Vérone pour y constater le nombre et la rareté des objets; en assurer la conservation, s'en faire effectuer te transport à Paris

Le ministre de l'Intérieur est chargé de l'exécution du présent arrêté.

Le Premier Consul Signé Bonaparte

Pour le Premier Consul

Le Secrétaire d'Etat

\section{DOCUMENT 12-2 AVRIL 1803}

Extrait des registres des délibérations du gouvernement de la République (AN, F/17/1537, sous-dossier Gazola).

$3 e$ Don [division du Ministère de l'Intérieur] enregé [enregistré] le $15 \mathrm{Germ}^{l}$ [Germinal]

$n^{\circ} 841$ 
Paris le 12 Germinal an 11 de la République.

Le Gouvernement de la République sur le rapport du ministre de l'intérieur arrête ce qui suit

\section{Article 1er}

La collection de poissons et autres objets pétrifiés, offerte au Premier Consul par la ville de Veronne est acceptée.

\section{Article $2 e$}

Le ministre de l'Intérieur est chargé de l'exécution du présent arrêté.

Le Premier Consul signé Bonaparte

Par le Premier Consul le Secrétaire d'Etat signé Hugues B. Maret contresigné Chaptal

\section{Pour copie conforme}

Le Secrétaire général

\section{DOCUMENT 13 - 7 AVRIL 1803}

Lettre de Gazola à Jean-Antoine Chaptal, ministre de l'Intérieur (AN, F/17/1537, sous-dossier Gazola).

\section{Au Ministre de l'Intérieur \\ Citoyen Ministre,}

J'ai l'honneur de vous instruire que depuis quelques semaines l'objet de ma mission est terminée [sic]: Ma Patrie me réclame ainsi que les fonctions de Président d'administration qui me sont confiées. Mais je ne puis éffectuer mon retour avant d'avoir obtenu l'acceptation par le Premier Consul du cabinet que je lui ai offert, et sa décision sur la réclamation que jai eu l'honneur de lui soumettre : ces deux solutions me sont indispensables, et vous sentez, Citoyen Ministre, combien il serait intéressant pour moi d'en être au plutôt le porteur ; il appartient, à Votre Excellence, d'obtenir pour moi auprès du Premier Magistrat de la République, les décisions dont le retard mempêche de vacquer aux affaires publiques et aux miennes, et ne fait que multiplier mes dépenses dans cette capitale, où je ne suis point aux frais du Gouvernement.

J'ai l'honneur de Vous saluer respectueusement

Paris ce 17 Germinal an 11

Jean Baptiste Gazola

Hôtel de Versailles rue Batave

\section{DOCUMENT 14-13 AVRIL 1803}

Copie de deux lettres de Jean-Antoine Chaptal, ministre de l'Intérieur, à Gazola (AN, F/17/1537, sous-dossier Gazola).

Il est prévenu que le gouvernement a accepté la collection de poissons pétrifiés offerte au 1er Consul par la ville de Verone.

$23 \mathrm{G}^{a l}$ [Germinal] an 11

Le Mtre au Citn Gazola, hôtel de Versailles, rue Batave

Je vous transmets ci-joint Citn, l'arrêté par lequel le gouvernement a accepté la collection de poissons pétrifiés, dont la ville de Verone vous a chargé de faire hommage au premier Consul.
Je prendrai incessamment les mesures nécessaires pour faire arriver cette collection à Paris. En attendant, je vous prie de vouloir bien être auprès de vos concitoyens, l'organe de mes remerciemens particuliers ; c'est à leur offre que notre cabinet d'hist: naturelle devra le complément de ce qu'il possédait déjà dans ce genre de curiosité.

$$
\text { Je v.s. [vous salue] }
$$

Transmission de l'arrêté qui lui accorde une gratification annuelle de $2500 \mathrm{fr}$.

$23 \mathrm{G}^{\mathrm{al}}$ [Germinal] an 11

$$
\text { Le Mrre au Citn Gazola. }
$$

Je m'empresse, Citn, de vous transmettre une ampliation de l'arrêté par lequel il vous est accordé sur les fonds du ministère de l'Intérieur une gratification annuelle de deux mille cinq cent francs, en acquit de ce qui restait dî sur le prix de la collection de poissons pétrifiés que vous avez cédée en l'an 5 au gouvernement français.

Vous voudrez bien prévenir votre fondé de pouvoir qu'il devra se présenter à la 4 e division de mon ministère le premier jeudi de chaque mois ; il y sera informé du jour où il pourra recevoir du Trésor public les arrérages mensuels de cette gratification.

$$
\text { j.v.s. }
$$

\section{DOCUMENT 15 - SANS DATE [FIN AVRIL-DÉBUT MAI 1803]}

Rapport d'un agent du ministère de l'Intérieur (AN, F/17/1537, sous-dossier Gazola).

On propose de consulter le M. d'hist. nat. [Muséum d'Histoire naturelle] sur le choix du comre [commissaire] qu'on pourrait charger du transport de la collection de poissons petrifiés dont la ville de Verone a fait hommage au 1er Consul Rapport

Le gouvt [gouvernement] ayant accepté la collection de poissons et autres objets petrifiés, offerte au $1^{\text {er }}$ consul par la ville de Vérone, il est d'autant plus nécessaire d'assurer le transport de cette seconde collection, que les caisses qui contenaient la lère arrivèrent, dans le tems, à Paris toutes brisées.

Pour présider à l'emballage, à l'encaissement et même à l'envoi des objets dont il s'agit, un comre [commissaire] instruit et zélé parait devoir être envoyé à Verone, et il convient qu'avant de le charger de cette mission le ministre sache quel est, et pourquoi, le traitement qu'on peut lui allouer.

On propose en conséquence de consulter les administrateurs $d u$ museum d'hist. nat. sur le choix du comre qu'on pourrait envoyer à Veronne pour le transport de la collection acceptée par le ler Consul, et sur le montant qu'il conviendrait.

Note écrite d'une autre main dans la marge du rapport : Le ministre a [arrêté] sur la lettre du Cen Bosc, la décision qui lui accorde $4000 \mathrm{f}$. pour la mission dont il est chargé. 


\section{DOCUMENT 16 - SANS DATE [FIN AVRIL-DÉBUT MAI 1803]}

Brouillon d'un arrêté de Jean-Antoine Chaptal, ministre de l'Intérieur (AN, F/17/1537, sous-dossier Gazola).

\section{Le Mine de l'Intérieur}

Vû l'arrêté du Gouvt du 11 Germinal dernier, portant que "la collection des poissons et autres objets petrifiés offerte au 1er Consul par la ville de Verone, est acceptée:"

Arrête ce qui suit:

Le Cen Bosc, naturaliste, est nommé pour aller recevoir au nom du Gouvernement la collection offerte par la ville de Verone et pour présider à son encaissement et prendre toutes mesures nécessaires pour que le transport jusqu’à Paris, s'effectue avec promptitude et sureté.

À Paris le...

\section{DOCUMENT 17 - 10 MAI 1803}

Copies d'une lettre et d'une note de Gazola aux professeurs administrateurs du Muséum national d'Histoire Naturelle, envoyées au ministre de l'Intérieur (AN, F/17/1537, sousdossier Gazola).

Citoyens Administrateurs,

Le Gouvernement a accepté l'offre que je lui ai faite au nom de la ville de Véronne, de tous les poissons fossiles qui manquent à votre collection.

J'ai, en conséquence, l'honneur de vous remettre quelques objets que j'avais apportés avec moi, dont la note est ci-jointe, et qui étaient destinés pour le Muséum; le Ministre de l'Intérieur a nommé M. Bosc, pour se rendre à Véronne, afin d'inventorier et faire arriver cette collection à Paris.

Comme il est important, Citoyens Administrateurs, de completter la riche collection que vous possédez déjà, \& quill est de l'intérêt de la science et de l'ouvrage qu'on publie sur cette collection, et qui est fort avancé, que les originaux soient réunis dans un Monument public, consacré aux Sciences, et que je le desire vivement.

J'ai l'honneur de vous prier d'inviter le Ministre, à décider, dès-à-présent, si la collection que jai offerte au nom de ma Patrie, servira de suite et de complément à celle du Muséum, et dans ce cas, le Ministre voudra bien charger M. Bosc, de sentendre, à ce sujet, avec l'administration du Muséum, afin quil prenne une note éxacte de tous les poissons que le Muséum possède, pour la comparer, lorsquil sera arrivé à Véronne, avec les poissons que j’ai dans la mienne, afin de n'envoyer à Paris que ceux qui y manquent, qui sont en grand nombre à la vérité; mais il faut éviter d'envoyer des doubles dont le Muséum n'a déjà qu'un très-grand nombre.

D'ailleurs, je n'ai offert moi-même à la ville de Véronne pour être donné au Gouvernement Français, que les poissons nécessaires, pour completter ceux qui manquent à la grande collection du Muséum.
Paris ce 20 Floréal an onze

J'ai l'honneur de vous saluer signé Jean-Baptiste Gazola

Pour copie conforme

A. Brongniart secrétaire

Note accompagnant la lettre précédente :

Note des objets appartenant au Muséum du Citoyen Gazola cédés à sa Patrie et offerts au Gouvernement Français

3 morceaux de pierres contenant des poissons \& plantes \&o fossiles, insérés dans une seule caisse, et qui proviennent de la formation de la Montagne d'où l'on tire ces fossiles, et des accidents de la pétrification. Morceaux précieux.

Un poisson à double empreinte de genre Office, de belle grandeur et colorié en taches quarrées et rondes. Exemple unique

Une plume

Un Oestrus de Mer

Un Olturia à double empreinte

Un insecte

Quatre empreintes de petits crabes

Cinq fleurs

Pour copie conforme

A. Brongniart

\section{DOCUMENT 18 - 16 MAI 1803}

Lettre (numéro d'enregistrement 1491) des professeurs administrateurs du Muséum national d'Histoire Naturelle (cosignée par René Desfontaines et Alexandre Brongniart) à JeanAntoine Chaptal, ministre de l'Intérieur (AN, F/17/1537, sous-dossier Gazola).

Paris, le 26 Floréal an Onze de la Republique Les Professeurs Administrateurs du Muséum national d'Histoire Naturelle, au Ministre de l'Intérieur,

\section{Citoyen Ministre,}

Nous avons l'honneur de vous adresser copie d'une lettre de $M$. le Comte de Gazola[6] par laquelle il nous engage à vous prier de décider si la collection de poissons pétrifiés quill a offerte au Gouvernement, servira à completter [sic] celle qui est déposée dans les Galeries du Muséum. Dans ce cas, il desire que le Citoyen Bosc que vous avez chargé d'aller à Vérone pour inventorier et faire arriver à Paris cette collection, s'entende avec nous et prenne note exacte de ce que le muséum possède déjà en ce genre, pour n'envoyer ici que les objets qui nous manquent. Dès que nous aurons reçu votre réponse nous la communiquerons à $M$. de Gazola, à moins que vous ne jugiez à propos de la lui faire parvenir directement

Salut \& Respect

Desfontaines directeur

A. Brongniart secrétaire

6 Il s'agit du document 17 (Annexe 1). 
Note en marge de la lettre écrite par un agent du ministère de l'intérieur, 3e division :

Ils [les professeurs du Muséum] adressent copie d'une lettre de M. le Comte de Gazola par laquelle il les engage à prier le Ministre de décider si la collection de poissons pétrifiés qu'il a offerte au gouvernement servira à completter celle qui est deposé dans les galeries du muséum.

\section{DOCUMENT 19 - 18 MAI 1803}

Brouillon d'une lettre de Jean-Antoine Chaptal, ministre de l'Intérieur, à Louis Augustin Guillaume Bosc (AN, F/17/1537, sous-dossier Gazola).

\section{8 floreal an 11}

\author{
Au C. Bosc
}

J'ai fait choix de vous, citen, pour aller à Veronne recevoir la collection de poissons pétrifiés que cette ville vient d'offrir au 1er Consul. Je ne doute point de l'intérêt avec lequel vous remplirez une mission dont le principal objet est d'assurer la conservation d'objets précieux pour la science que vous cultivez avec tant de succès.

J'ai arrêté qu'il vous serait alloué pour vos frais de voyage, une somme de $4.000 \mathrm{f}$. Vous pouvez dès à présent vous présenter à la $4^{e}$ division de mon ministère pour y recevoir la lettre d'avis sur la quelle vous toucherez cette somme au Tresor public.

Voici l'état des pièces que vous trouverez jointes à cette lettre:

$1^{\circ}$. Une ampliation de l'arrêté du Gouvt du 11. Gal [germinal], portant acceptation de la collection des poissons pétrifiés, offerte par la ville de Vérone.

$2^{\circ}$. L'arrêté par le quel je vous ai nommé commissaire pour recevoir, faire encaisser et transporter cette collection.

$3^{\circ}$. Une instruction qui vous servira de règle dans votre mission, et que je crois propre à en faciliter le succès.

Instruction pour le commissaire chargé de recevoir, au nom du Gouvernement la collection de poissons pétrifiés offerte au 1er consul par la ville de Veronne.

1. Avant son départ de Paris, le commissaire se concertera avec les administrateurs du Muséum d'histoire naturelle, sur les moyens d'éviter les accidens qui ont nui à la première collection de poissons qui a été transportée à Paris.

2. Pendant son voyage, sil découvre dans les collections qu'il pourra visiter des objets qui lui paraitront manquer dans les collections du Museum de Paris, il en informera les professeurs administrateurs de cet établissment. En général il est invité à leur faire part de toutes les observations geologiques, botaniques, $\delta \mathrm{c}$. qu'il pourra avoir occasion de recueillir.

3. à son arrivée à Verone, le commissaire après avoir fait reconnaitre son titre par les autorités de la ville, et leur avoir remis une ampliation du don offert par la ville, soccupera de constater par un inventaire, le nombre, l'etat de conservation et la rareté des objets qui composent la collection. Il enverra un double de cet inventaire au ministre de l'intérieur.

4. Le commissaire contractera tous les marchés nécessaires pour l'encaissement des objets et choisira pour leur transport la voie et les moyens qui lui paraitront les plus propres à en assurer la conservation. Il enverra au ministre des doubles de ces marchés pour que les payements s'executent aux époques qui y seront déterminées. $5^{\circ}$. Le commissaire visitera avec soin, la montagne de Vestena Nova d'où sont tirés les poissons pétrifiés. Il donnera aux professeursadministrateurs du Museum d'hre $n^{l e}$, tant sur cette montagne que sur les environs, tous les renseignemens et les détails qui lui paraîtront propres à avancer la science de la géologie.

Fait à Paris, le ...

Le Mre [Ministre]

\section{DOCUMENT 20 - 25 MAI 1803}

Lettre (numéro d'enregistrement 276) de Louis Augustin Guillaume Bosc à Jean-Antoine Chaptal, ministre de l'Intérieur (AN, F/17/1537, sous-dossier Gazola).

\section{Paris 5 Prairial an XI de la Republique Citoyen Ministre,}

J'ai reçu avec infiniment de reconnoissance la lettre dont vous mavez honoré le 28 du mois dernier [28 floréal an 11] à laquelle était joint $1^{\circ}$. l'arrêté du Gouvernement du 12 Germinal précédent, qui accepte la collection de poissons pétrifiés offerte par la ville de Veronne au premier Consul; $2^{\circ}$. Celui par lequel vous me nommez pour aller recevoir et apporter cette précieuse collection; $3^{\circ}$. L'instruction que vous avez fait rediger pour me regler dans mes opérations.

Cette marque de votre confiance m'était d'autant plus agréable que nous nous trouvons à l'époque de l'année la plus favorable pour faire sur la route des observations zoologiques et botaniques et en conséquence je prevois mes dispositions pour partir le plus promptement possible, mais une circonstance imprevue me force d'attendre de nouveaux ordres de votre part.

La collection de poissons pétrifiés cedée par M. de Gazola à la République m'était connue, mais j'ignorais qu'il en eut formé une autre et j'avais été, par la, porté à croire, en recevant votre lettre, que la ville de Veronne en avait une en propre; aussi lorsque, conformement avec des articles de votre instruction, je me suis transporté au Museum d'histoire naturelle, ai-je été fort surpris d'apprendre que la collection cedée et que je devais apporter, n'appartenait réellement pas à la ville de Veronne, mais a $M$. de Gazola qui l'avait offerte au Premier Consul au nom de la ville. J'ai appris, de plus, que M. de Gazola était encore à Paris et avait même adressé, depuis peu, une lettre qui vous avait été envoyé, et dans laquelle il semblait restreindre l'etendue du don accepté par le Gouvernement et y mettre des conditions. D'après cela jai vu M. de Gazola qui m'a en effet confirmé qu'il avait eu intention de ne donner au Gouvernement, pour le Muséum d'histoire 
naturelle que les articles de sa collection qui ne sy trouvent pas encore et qu'en conséquence il fallait d'abord constater tout ce qui y était et ensuite attendre que la mission dont il est chargé, lui permit de retourner à Veronne, pour choisir avec moi, dans la sienne, les objets qui nous manquent; c'est-à-dire remettre mon voyage à l'hiver prochain.

Je ne connois point les termes de l'offre faite au nom de la ville de Veronne, mais ceux de l'arrêté des Consuls que vous m'avez transmis, ne me permets pas d'adopter l'interprétation de $M$. de Gazola. J'étais bien disposé et je l'avais même commencé avant de voir M. de Gazola, à prendre un croquis des poissons les plus caractérisés de la galerie du Muséum, pour me guider dans le choix de ceux que je devais rapporter, mais je ne pensais pas que ce travail dut être rigoureux et qu'il du être la base de mon opération. Je croyais devoir apporter tous les échantillons qui par leur belle conservation méritaient, à mes yeux, les frais du transport, quoi qu'existant déjà dans la collection du muséum.

Je vous prie, en conséquence, Citoyen Ministre, de vouloir bien vous faire remettre sous les yeux les pieces fondamentales de cette affaire, vous entendre avec $M$. de Gazola, et me faire part de votre détermination ultérieure.

$$
\begin{gathered}
\text { Salut et respect } \\
\text { Bosc }
\end{gathered}
$$

rue des maçons Sorbonne

\section{DOCUMENT 21 - 1ER JUIN 1803}

Rapport de Jean-Pierre Barbier-Neuville (1754-1822), chef de la 3 e division du Ministère de l'Intérieur, à Jean-Antoine Chaptal, ministre de l'Intérieur (AN, F/17/1537, sous-dossier Gazola).

Proposition d'arrêter que la collection de poissons pétrifiés, offerte au Gouvernement pour la ville de Vérone, sera transportée à Paris dans sa totalité.

Paris, le 12 Prairial an 11 de la République une \& indivisible. Rapport présenté au Ministre de l'Intérieur.

L'administration du Museum d'histoire naturelle adresse copie d'une lettre par laquelle M. Gazola demande que le Ministre décide si la collection de poissons pétrifiés, offerte par la ville de Vérone au Gouvernement français, servira à compléter celle qui est déposée dans les galeries du musée. Dans ce cas, il désire que le Cen Bosc, chargé d'aller inventorier cette collection et de la faire arriver à Paris, prenne une note exacte de ce que le muséum possède déjà en ce genre, pour n'envoyer que les objets qui manquent ici.

Le Ministre ne doute pas qu'il ne se trouve beaucoup de doubles dans la collection de Vérone. Il sait aussi qu'en les comparant avec ceux de Paris, on peut y trouver des différences qui les fassent préférer. D'ailleurs on formerait de ces doubles, des collections partielles qui seraient accordées à d'autres établissements publics. Tout ce qu'on pourrait en écarter, et même avec quelque ménagement, ce serait ces morceaux composés de parties brisées de différents poissons (à supposer qu'il en existe de pareils dans cette collection) peut-être y aurait-il de l'avantage à les emporter tous, ne fut-ce que pour prouver que c'est ce genre de fourberie qu'il faut attribuer la persuasion où l'on est que les montagnes $d u$ Véronais présentent un plus grand nombre de poissons inconnus aux nomenclateurs qu'il ne s'en trouve réellement.

Quoiqu'il en soit, M. Gazola remarque dans sa lettre à l'adon qu'il n'a offert lui-même à la ville de Vérone, que les poissons nécessaires pour completter la grande collection du Muséum.

Cette restriction dont on n'a aucune connaissance, n'a pas été mentionnée dans l'offre faite au Premier Consul, ni dans les lettres que M. Gazola a adressées à ce sujet tant au Ministre de l'Intérieur qu'au Ministre de la République italienne.

Je propose donc à Votre Excellence d'arrêter que la collection de poissons pétrifiés, offerte au Gouvernement Français par la ville de Vérone, sera transportée à Paris dans sa totalité. L'admon du muséum d'histoire naturelle sera invitée à donner connaissance de cette décision à M. Gazola.

P.S. Ce rapport était terminé, lorsque le Cen Bosc a écrit au Ministre pour lui témoigner toute sa surprise d'une interprétation qu'il ne peut adopter d'après l'arrêté des Consuls qui lui a été transmis. Son intention était bien de prendre un croquis des poissons les plus caractérisés de la galerie du Muséum pour se guider dans le choix de ceux qu'il devait apporter, mais il ne pensait pas que ce travail dît être rigoureux, et qu'il dît être la base de son opération. Il croyait devoir apporter tous les échantillons qui, par leur belle conservation, mériteraient à ses yeux, les frais du transport, quoiqu'existant déjà dans la collection du Muséum.

Au surplus il se disposait à partir dans ce moment favorable aux observations zoologiques et botaniques, mais M. Gazola lui a dit qu'il fallait attendre que la mission dont il est chargé ici, lui permît de se rendre à Vérone, pour y choisir ensemble ce qui manque au Musée de Paris, c'est-à-dire remettre le voyage du Cen Bosc à l'hiver prochain.

D'après ces observations, on croit devoir persister dans la proposition ci-dessus.

$$
\begin{gathered}
\text { Le chef de la 3e Division } \\
\text { J.P.Barbier-Neuville }
\end{gathered}
$$

\section{DOCUMENT 22-8 JUIN 1803}

Brouillons de deux lettres de Jean-Antoine Chaptal, ministre de l'Intérieur, l'une à Bosc, l'autre à l'administration du Muséum d'Histoire naturelle (AN, F/17/1537, sous-dossier Gazola).

Il est prévenu que la collection de poissons pétrifiés, offerte au premier Consul par la ville de Vérone, soit être transportée à Paris dans sa totalité

$19 p^{a l}$ [prairial] an 11. Le Mtre au Citn Bosc \&oc. 
J'ai reçu, Citn, vos observations sur la proposition que fait $M^{r}$ Gazola de n'apporter de la collection de poissons pétrifiés, offerte au premier Consul, que les articles qui manqueraient à celle du Museum d'hist: naturelle.

Cette restriction que Mr Gazola semble mettre aujourd'hui à l'hommage de la Ville de Vérone ne peut être admise. D'ailleurs sil existe des doubles dans cette collection, il est probable qu'il s'y trouvera des différences assez sensibles pour les faire préférer. Dans tous les cas, ils serviraient ici à former des collections partielles qui seraient accordées à d'autres établissements publics. En conséquence, j’ai arrêté qu'elle serait transportée à Paris dans sa totalité, et je m'en refere à cet égard, à ma dernière lettre, en vous invitant à partir aussitôt que les circonstances vous le permettront.

Lorsque vous serez à Vérone, je vous prie vous voudrez bien vous présenter chez le $D^{r}$ Thouvenel qui vous remettra, sous votre récépissé, 300 exempl: [exemplaires] des 4 premiers volumes du traité qu'il a publié sur le climat de l'Italie, et pour lequel le gouvernement a souscrit. Je vous prie de les faire encaisser de manière à ce qu'il ne leur arrive aucun inconvénient, et de joindre cet envoi à celui de la collection.

$$
\text { J.v.s. [Je vous salue] }
$$

Lettre à l'administration du Muséum d'Histoire naturelle :

Le Mrre a arrêté que la collection de poissons pétrifiés, offerte au Ier Consul par la ville de Vérone, serait être transportée à Paris dans sa totalité

$$
\text { Le Mtre }
$$

à l'admon du Museum d'hist: naturelle

J'ai reçu avec votre lettre, Citns, celle par laquelle $M^{r}$ Gazola desire savoir si la collection de poissons pétrifiés, offerte au $G^{t}$ [Gouvernement] par la Ville de Vérone, servira à completter celle qui est déposée dans les galeries du Museum, et dans ce cas, il demande que le Citn Bosc prenne une note exacte de ce que le museum possède déjà en ce genre, pour n'envoyer ici que les objets qui lui manquent.

Je ne doute pas qu'il ne se rencontre beaucoup de doubles dans la collection de Vérone, mais il est probable qu'en les comparant avec ceux de Paris, on y trouvera des différences qui peuvent les faire préférer, et dans tous les cas, ils serviraient ici à former des collections partielles qui seraient pourraient être accordés à d'autres établissements publics.

Mr Gazola ajoute qu'il n'a offert lui-même à la ville de Vérone que les poissons nécessaires pour completter la grande collection du Museum. Cette restriction dont je n'ai nulle connaissance, et qui n'a été mentionnée ni dans l'offre au premier Consul, ni dans les lettres qui m'ont été adressées à ce sujet, ne peut être admise. En conséquence j'ai arrêté que la collection dont il s'agit serait transportée à Paris dans sa totalité. Je vous invite, Citns, à vouloir bien instruire $M^{r}$ Gazola de cette décision.

$$
\text { J. v. s. }
$$

\section{DOCUMENT 23 - 17 AOÛT 1803}

Copie d'une lettre de Louis Augustin Guillaume Bosc remise aux citoyens administrateurs de la municipalité de Vérone. Ce document est accompagné d'une copie d'une lettre de Jean-Antoine Chaptal, ministre de l'Intérieur, à Bosc, en date du 19 prairial an 11 (8 juin 1803) (AN, F/17/1537, sous-dossier Gazola).

\section{Verone 17 aoust 1803}

Le naturaliste Bosc, commissaire du gouvernement françois, aux citoyens administrateurs de la Municipalité de Verone Italienne.

\section{Citoyens administrateurs}

Lorsquej'êेs [sic] l'honneur de vous voir, et de vous faire part, il y a environ un mois, de la mission dont jeétois [sic] chargé auprès de vous, je n'ai pas cru de ma delicatesse [sic] de vous parler de la restriction que M. Gazola avoit voulu mettre au don qu'il a fait, en votre nom, au Premier Consul de poissons fossiles de sa collection, parceque [sic] je ne presumois [sic] pas qu'après les lettres que le Ministre de l'Intérieur a ecrit a l'administration du Museum, et a [sic] moi, lettres qui lui ont été communiquées en original, il voulut entreprendre de mettre encore quelqu'obstacle a la remise de la collection dans sa totalité. Il paroit [sic] cependant que je m'étois trompé, et que, d'après la lettre, dont vous avez bien voulu me procurer hier la lecture, il persiste dans l'interpretation [sic] donnée par lui avant mon depart au texte de la note qu'il a remis en votre nom au Premier Consul. Je dois vous repetter [sic] aujourd'hui par ecrit [sic] ce que je vous ai dit hier de vive voix, Citoyens administrateurs, c'est qu'il ne m'est plus permis de discuter cette matière avec vous. La lettre du Ministre de l'Intérieur, dont j'ai l'honneur de vous envoyer copie, me faisant une loi de reclamer auprès de vous la collection entiere, ou de ne recevoir de vous que la collection complette existant chez $M$. de Gazola a ce que vous m'avez annoncée, mais devant sy trouver facilement.

J'ai donc lieu de croire que vous voudrez bien effectuer les promesses, que vous m'avez fait hier, \& vous occuper aujourd'hui des moyens de me mettre en etat de remplir ma mission en prenant les arretés necessaires [sic] pour me mettre en possession, et faire faire sous ma direction les caisses, et les travaux d'emballage propres a [sic] assurer la conservation de votre precieux [sic] don, car il est certainement de votre dignité de ne pas affaiblir le merite [sic] du sacrifice que vous avez fait en prolongeant plus longtems [sic] l'epoque de sa remise sous des pretexes qui pourroient paroitre [sic] peu fondés aux personnes malintentionnées pour vous, ou pour les interets [sic] des citoyens que vous representez [sic]. D'ailleurs les ordres du Ministre, mes occupations, et les approches de l'hiver, pour le passage des Alpes, ne me permettent pas de séjourner encore bien longtems en Italie.

Salut et respect

Bosc

Copie de la lettre du Ministre de l'Intérieur de la République françoise au Citoyen Bosc commissaire du gouvernement auprès de la Municipalité de Verone italienne, en date du 19 Prairial an 11 de la Rep. 
J'ai reçu, citoyen, vos observations sur la proposition, que fait M. Gazola de n'apporter de la collection de poissons petrifiés [sic], offerte au Premier Consul, que les articles qui manquoient a [sic] celle du Museum de l'histoire naturelle.

Cette restriction que M. Gazola semble mettre aujourd'buy [sic] a [sic] l'hommage de la ville de Veronne, ne peut etre [sic] admise. D'ailleurs il existe des doubles de cette collection, il est probable qu'il s'y trouvera des différences assez sensibles pour les faire preferer [sic]. Dans tous les cas, ils serviroient ici a former des collections partielles, qui seroient [sic] accordées a d'autres etablissements publics. En conséquence j'ai arrêté qu'elle seroit [sic] transportée a [sic] Paris dans sa totalité, et je m'en réfere a [sic] cet egard a ma dernière lettre en vous invitant a [sic] partir aussitôt que les circonstances vous le permettent

Pour copie conforme

$$
\text { Je vous salue }
$$
Chaptal Bosc

\section{DOCUMENT 24-18 AOÛT 1803}

Lettre des officiers de la municipalité de Vérone à Gazola. Ce document était joint à la lettre de Gazola à Napoléon Bonaparte (Annexe 1[doc. 26]) (AN, F/17/1537, sous-dossier Gazola).

Au citoyen JeanBaptiste Gazola

Hotel de Versailles, rue Batave $N^{\circ} 409$ Paris

Verona, li 18 Agosto 1803 anno 2. La Municipalità di Verona

Al Cittadino Gio: Batta: Gazola, sue Presidente, e Delegato al Po [Primo] Console della Repubblica Francese, e Presidente della Repubblica Italiana.

Non è solamente certo, ma ragionevole ancora, che il generoso vostro dono di pesci alla patria consista unicamente nel da lei desideratissimo completamento del Museo del P:C: [Primo Console], e Presidente, ne mai vi abbiamo chiesta perciò la totalità della vostra collezione, la quale in parte formerebbe una duplicità superflua de pesci stessi nell'anunciato museo, la cui singolarità di nulla avere di doppio lo renderebbe piu grato all'occhio di qualunque osservatore.

Tanto abbiamo esposto anche a M. Bosc, all'occasione, che, ritornato jer l'altro dal suo viaggio, ci diede l'onor di vederlo. Gli comunicammo altresi la stessa vostra lettera 31 Luglio, e le copie della vostra offerta al Primo Console, e del foglio con cui arete illustrata la cosa agli Administration del Museo.

Ci diede a leggere il mentovato Bosc la risposta, che nel proposito aveva ricevuta da codesto Ministero dell'Interno, ed esterno in oltre personalmente la sua insistenza di ricevere la totalità della vostra collezione, secondo il senso, chegli da alla vostra offerta sud:ta [suddetta] : soggiunse che non si scherza col Governo Francese, e che, in difetto della consegna totale a lui dovuta dalla nostra Municipalità, egli partirebbe senza riceverne parte alcuna. Attribui, in certo modo, a vostra colpa che ne abbiate qui spedita una nota supplente in vostra assenza all'apurimento di quest'affare. Non ommise di dichiararci, che appunto qualle duplicità, che costi fossero per essere superflue al museo, sono opportune a Luciano, e ad altri fratello del Primo Console, non che in altri luoghi dell'esteto territorio francese. Jeri finalmente ci fece la domanda formale di essa intera vostra collezione, rimettendoci anche copia della risposta, chebbe da codesto Ministero dell'interno: d'entrambe vi accludiamo le copie, a vostro lume.

Necessitati perciò dalla prudenza di non lasciar partire disgustato Mr Bosc, perche il disgusto suo verrebbe sostenuto dal prelodato ministero dell'Interno, non consaperale delle sincere nostre intenzioni, abbiamo calcolata piuttosto la vostra generosità verso la Patria, e la di lei naturale premura, che un cittadino di merito si grande quale appunto voi siete, non debba possibilmente essere esposto a dispiaceri, che sarebbero in fatti a lei non meno che a noi d'acerrimo, insuperabil dolore.

Spinti da principi si giusti, abbiamo detto a $M^{r}$ Bosc, ch'egli avrà tosto quanto ci riescivà rinvenire della vostra colleione; $e$ che di quanto si trovasse, per avventura, fuori di casa vostra, del che siamo affatto ignari, non solo noi, ma la vostra famiglia pur anche, ne seguirà la spedizione a Parigi subito che vi sarete restituito alla Patria.

Quindi è, che invitato da noi il fratello vostro, Cittadino Giuseppe, ha pienamente approvata la nostra direzione; e seco lui abbiamo delegato il cittadino Rotari, municipale, a soddisfar la domanda di Mr Bosc, rappresentante codesto ministro.

Avesse almeno inteso il prelodato ministro di dare un maggior merite alle generose vostre intenzioni verso la Patria, ad all divozione della nostra comune verso il nostro Presidente, nel privarti anche di ciò, ch'è ultraneo al Museo di Parigi, unico oggetto della nostra premura.

Abbiamo il contento di ripetervi i sentimenti sinceri della distinta nostra gratitudine, estima

Crema Giovio

\section{DOCUMENT 25 - 29 AOÛT 1803}

Lettre de Louis Augustin Guillaume Bosc à Jean-Antoine Chaptal, ministre de l'Intérieur (AN, F/17/1537, sous-dossier Gazola).

Verone le fructidor an IX ${ }^{[7]}$ de la Rep. 29 aout 1803 Citoyen Ministre,

Je ne vous ai pas depuis longtemps rendu compte de ma marche en Italie pour ne pas multiplier inutilement mes lettres, n'ayant rien d'important à vous apprendre.

La dernière que j'ai eu l'honneur de vous écrire vous annoncoit que j'allois passer sur les bords de la mer adriatique et dans la montagne du Vicentin, le temps nécessaire pour que la Municipalité de Verone puisse recevoir de M. Gazola les renseignements

7 C'est une erreur, il s'agit de l'an XI. 
propres à les mettre a portée de satisfaire à l'arrêté du Consul. J'ai rempli vos vuës dans cette tournée ou j'ai visité le mont Bolca, la carrière des poissons fossiles, et plusieurs collections intéressantes, mais j'ai peu ramassé d'objets a raison de la rapidité avec laquelle je l'ai faite et de son époque trop avancée.

Je suis rentré a Verone il y a aujourd'hui quinze jours et me suis présenté à la Municipalité qui avait enfin reçu des lettres de M Gazola. Elle a d'abord voulu faire quelques difficultés pour me remettre la collection entiere mais sur la vue de votre lettre, dont je lui ai remis copie, elle s'est éxécutée complettement et je n'ai eu qu'a me louer de ses procédés. J'ai ensuite travaillé au Catalogue qui renferme 191 articles la plus part double, c'est-à-dire composés par deux empreintes opposées. Plusieurs sont d'une grande importance et ne se trouvent pas dans la collection du Museum mais leur nombre n'est pas aussi considérable que M. Gazola l'avoit annoncé. Je vous envoie un des exemplaires de ce catalogue sur lequel j'ai grossierement figuré chaque espece de poisson afin qu'on puisse se former une idée de sa forme. L'autre est resté, revetu de mon récépissé entre les mains de la municipalité. Cela fait j'ai procédé a l'ambalage [sic] que j’ai fait presque tout seul et que je crois conditionné de manière a assurer l'arrivée des pièces en bon état, jusqu'à Paris. La municipalité a payé les caisses et autres objets de sorte que je n'ai déboursé qu'une petite somme principalement en gratifications aux ouvriers et domestiques.

J'ai également reçu des mains du docteur Thouvenel[8] les trois cent exemplaires de son ouvrage sur le climat de l'Italie[9] et je lui en ai donné un reçu conformement à vos ordres. Ces exemplaires ont été emballés dans des caisses particulieres ainsi que quelques uns d'un autre ouvrage du même que vous ne m'aviez pas chargé de recevoir mais qu'il désire faire connaitre en France et a l'occasion duquel il a du vous écrire. Il est intitulé la guerre de 10 ans et a trait à ses idées sur le galvanisme \&oc[10].

Tous ces objets sont renfermés dans trente trois caisses pesant 6978 livres poids d'Italie [11].

Je n'ai pu faire usage à Verone du crédit que vous mavez ouvert sur votre ministere pour payer les frais de transport, mais $j$ 'ai $p u$ y emprunter, en mon nom, $600 \mathrm{f}$. payable dans Paris immédiatement après mon retour, et qui ont plus que suffit pour les dépenses d'emballage et le transport de Vérone à Milan, lieu ou jespere trouver plus facilement des entrepreneurs pour la suite du voyage. Je vous porte le compte de ces dépenses.

J'aurai soin de vous instruire de Milan de la suite de mes opérations.

Salut et respect

\section{Bosc}

8 Pierre Thouvenel (1745-1815), médecin français originaire des Vosges.

9 (Thouvenel 1797-1798)

10 (Thouvenel 1802)

11 Suivant les villes italiennes, la livre poids avait des valeurs différentes, par exemple, $0,28 \mathrm{~kg}$ à Mantoue, environ $0,33 \mathrm{~kg}$ à Milan, Parme, Florence, Bergame et Livourne, et jusqu’à $0,48 \mathrm{~kg}$ à Venise. 6978 livres devaient donc représenter entre 2 et 3 tonnes (Palaiseau 1816).

\section{DOCUMENT 26 - FIN AOÛT 1803}

Lettre de Gazola à Napoléon Bonaparte, Premier Consul et Président, Napoléon Bonaparte, écrite en août 1803 (après le 18) (AN, F/17/1537, sous-dossier Gazola).

"Le C. Gazola se plaint de ce que le C. Bosc a enlevé de chez luy une collection de poissons qui ne fasoit pas partie du don de la Ville de Veronne»[12]

Citoyen Premier Consul et Président,

Vous daignâtes il y a quelques mois accepter une offre que la Commune de Vérone avait pris la liberté de vous faire par mon organe.

Heureux d'avoir obtenu de vous cet acte de bonté pour moi et mes Concitoyens, j'étais loin de prévoir qu'il deviendrait l'occasion d'une entreprise contre laquelle je serais forcé d'implorer votre justice et votre protection. C'est pourtant ce qui marrive, et souffrez que j'ose vous exposer le fait.

Quand il eut plu au Gouvernement français d'avoir en sa possession la collection de poissons et de plantes pétrifiées que j'avais formée, je conçus le dessein de la rendre aussi complette qu'il serait possible.

Je continuai donc mes recherches, et fouillant sans cesse dans la Montagne de Vestena-Nuova qui mappartient et qui est comme une mine inépuisable, je trouvai, en effet un grand nombre de nouvelles pièces qui ajoutées à la collection devaient la rendre la plus riche et la plus curieuse qu'il y ait en Europe.

Ma première idée avait été de vous les offrir moi-même; mais voyant que la commune de Vérone désirait vous présenter quelque hommage qui pût vous être agréable, je crus pouvoir lui en fournir le moyen, en lui cédant ce fruit de mes travaux, pour qu'il vous fût offert.

Après que vous ềtes bien voulu consentir de l'accepter, il fut question de prendre les arrangements convenables, pour que ces nouvelles pièces fussent livrées reçues et transportées en France.

Ce que je proposai dans cet objet, fut que quelqu'un des Membres de l'administration du Muséum d'histoire naturelle fut chargé de se rendre à Vérone en même tems [sic] que j'y retrournerais. D'une part, en effet, il convenait que la commission d'aller recevoir fût donnée à quelqu'un qui connùt déjà la collection qui est actuellement à Paris. De l'autre ma présence à cette opération paraissait absolument nécessaire, vu que les pièces nouvellement recueillies sont toujours restées chez moi, mêlées, confondues dans l'immense collection de tous genres que j'ai formée et même avec beaucoup d'autres pièces de même nature, que je n'ai cessé de rassembler, soit pour mon propre usage, soit dans l'objet d'en enrichir les Cabinets de Pavie et de Bologne.

Mais, je ne sais pour quelle raison, le Ministre de l'Intérieure préféra de choisir le Cen Bosc, qui ne tient en aucune manière au Museum, et d'ailleurs, ce Citoyen, au lieu de m'attendre, comme je l'en priais avec instance, se hâta de partir pour l'Italie.

Quoiqu'il en soit, arrivé à Vérone, le Cen Bosc a prétendu quion devait lui livrer non pas seulement les pièces nouvelles que j’avais

12 Commentaire écrit d'une autre main en marge de la lettre. 
cédées à la commune et qui devaient être ajoûtées à celles qui sont déjà à Paris, mais encore toute ma propre collection, de quelque manière qu'elle fut composée.

En vain, on lui a représenté que jamais mon intention n'avait été de faire un pareil sacrifice, et que d'ailleurs en enlevant tout, il emporterait beaucoup de choses inutiles, puisqu'ily aurait jusque à cinq ou six exemplaires d'une même pièce. Il a répondu que j'avais offert ma collection, qu'elle avait été acceptée, qu'il n'était plus possible de restreindre le présent, et que sil se trouvait des doubles, triples exemplaires, on saurait bien les placer.

Il a fait plus. Il a exigé que tout lui fut remis sans mattendre, et menaçant, effrayant les officiers Municipaux, il les a déterminés à lui livrer indistinctement tout ce qui était chez moi, et même encore à s'engager d'envoyer à Paris tout ce que je pourrais avoir placé ailleurs que dans ma maison.

C'est ce que ces officiers me mandent eux-mêmes par une lettre du 18 de ce mois, de laquelle jose vous supplier de prendre lecture, et où ils mexpriment la peine qu'ils ont ressentie de ce procédé.

Ainsi donc, Citoyen Premier Consul, dans ce moment même l'on s'est emparé de tout le fruit de mes longs et pénibles travaux. Ainsi, en me privant de tout ce que j'ai recueilli, on a renversé tous les projets que je formais, soit pour le progrès de la science, soit pour l'utilité de nos établissemens publics. Ainsi, en un mot, l'on m'a porté un des coups les plus sensibles dont je pusse être frappé.

Mais une espérance me reste et assurément elle ne sera point trompée. C'est que votre justice, votre générosité ne souffriront pas que l'on me dépouille de cette manière. C'est que vous voudrez bien ordonner que tout ce qu'on m'a pris me soit rendu et que si même les caisses sont déjà parties de Vérone, elles soient arrêtées partout où elles se trouveront et reportées chez moi pour que jen sépare tout ce qui n'a point du être livré.

Certainement, je suis éloigné de vouloir restreindre un hommage qui vous a été offert et que vous avez daigné agréer. Certainement, je donnerai toujours avec le plus vif plaisir, tout ce qui peut contribuer à completter, à embellir la collection qui est à Paris, et je m'estimerai heureux qu'on veuille bien le recevoir. Mais je serai bien affligé, si l'on me privait du surplus.

Daignez, Citoyen Premier Consul et Président, recevoir avec bonté l'hommage de mon profond respect

Jean Baptiste Gazola

\section{DOCUMENT 27 - 14 SEPTEMBRE 1803}

Lettre de Louis Augustin Guillaume Bosc à Jean-Antoine Chaptal, ministre de l'Intérieur (AN, F/17/1537, sous-dossier Gazola).

\section{Milan 27 thermidor ${ }^{[13]}$ an XI}

Citoyen Ministre,

J'ai attendu quelques jours les suites de l'évènement dont je vous ai entretenu dans ma dernière lettre. Aujourd'hui que le

13 C'est une erreur, il s'agit du mois de fructidor hazard m'a fait rencontrer Gazola, je sais à quoi m'en tenir. En conséquence, je me dispose a retourner demain en France, par la route la plus directe, le St Gothard, que je désire visiter. Je viens d'écrire au Vice Président pour réclamer les caisses qui contiennent l'auvre de Thouvenel et de faire, avec les frères [...] un marché que vous trouverez cy joint pour leur transport jusqu'à Lyon.

\section{Salut et respect \\ Bosc}

\section{DOCUMENT 28 - 14 SEPTEMBRE 1803}

Lettre de Louis Augustin Guillaume Bosc à Giuseppe Angelo Saluzzo di Menusiglio ${ }^{[14]}$ (collection privée).

\section{Monsieur de Saluces}

Président de l'Accadémie des Sciences à Turin

Milan 27 fructidor an XI

J'avais espéré un moment, mon cher et respectable collègue d'être obligé de retourner à Turin, mais les circonstances ont changé et je puis éxécuter mon premier plan de passer par le St. Gothard sur lequel je suis bien aise de pouvoir jetter un coup d'oil. En conséquence je me vois forcé de vous adresser par la diligence un paquet de livres que Malacarné m'avait chargé de vous remettre. Des relations que vous m'avez données, la sienne a été la plus douce à mon coeur. Je ne dois jamais oublier toutes les marques de bienveillances dont il m'a comblé ainsi que son fils, mais je les rapporte cependant en partie à vous qui savez si bien captiver l'estime et l'attachement des personnes qui ont le bonheur de vous connaitre. Le voyage que j'ai fait ne m'a été agréable que sous le point vue de l'accueil que j'ai reçu des savans que j'ai été [...] de visiter car il ne m'a procuré que fort peu d'objets propres à avancer les progrès de la science que je cultive et je me suis considérablement déplu de perdre dans les auberges un temps que j'aurais eu mieux employé autre part. J'ai vu d'ailleurs le but de ma mission devenir inutile par les ordres du Premier Consul qui a fait arrêter à Milan les caisses de poissons fossiles de Bolca que je conduisais en France, par des motifs qu'il serait trop long de vous décrire.

Je vous prie de vouloir bien me rappeler au souvenir de nos collègues de qui j'ai reçu tant de marques de bienveillance pendant les trop courts moments que j'ai passé à Turin. Dites surtout à Giobert que je regrette de ne l'avoir pas pu voir plus souvent comme je me l'étais proposé. Je cite celui-ci seulement parce que je compte écrire aux autres à mon arrivée à Paris. J'ai l'honneur de vous renouveller les assurances de mon respectueux dévouement

$$
\text { Bosc }
$$

14 en français, Joseph-Ange, comte de Saluces de Menusiglio (1734-1810) 


\section{DOCUMENT 29-9 OCTOBRE 1803}

Lettre de Louis Augustin Guillaume Bosc à Jean-Antoine Chaptal, ministre de l'Intérieur (AN, F/17/1537, sous-dossier Gazola).

Citoyen Ministre,

Paris 16 vendemiaire an 12 de la Rep.

J'ai eu l'honneur de vous instruire successivement de toutes les opérations qui ont été la suite de la mission de [sic] vous avez bien voulu me charger auprès de la Municipalité de Veronne, et en dernier lieu de mon départ de Milan sans la collection de poissons fossiles que je devais amener avec moi, attendu qu'elle avait été arrêtée dans cette ville par ordre du Premier Consul. Me voici de retour. Je n'ai plus qu'a [sic] vous remercier de la confiance que vous m'avez accordé et a [sic] vous demander le remboursement de quelques dépenses qui ne devoient pas être imputées sur la somme que vous mavez accordée pour frais de voyage. J'en joins ici la note. Je désire qu'il soit possible d'en toucher promptement le montant attendu qu'il fait partie d'une somme de $600 \mathrm{f}$. que j'ai emprunté à Veronne et que je me suis engagé de payer à mon arrivée à Paris.

Je pars demain pour me mettre en activité de service dans le poste d'inspecteur des pépinieres de Versailles que je dois à votre bienveillance. J'ai pris, en cette qualité, avec les professeurs du jardins de botanique que j'ai visités pendant mon séjour en Italie, l'engagement de leur faire quelques envois de graines ou de plantes des espèces qui leur manquent. Je vous prie de vouloir bien mautoriser a le remplir. Je vous transmettrai la note de mes envois lorsqu'ils seront effectués.

\section{Salut et respect}

Bosc

\section{DOCUMENT 30 - SANS DATE [OCTOBRE 1803]}

Rapport d'un agent du ministère de l'Intérieur au ministre (AN, F/17/1537, sous-dossier Gazola).

On propose d'ordonner au Mtre d'ordonner le payement d'une somme de 479f50c avancée par le Citn Bosc pour l'emballage de la collection de poissons pétrifiés de Vérone. Il voudra bien en même tems bien prononcer sur la seconde demande du Citn Bosc, tendant à envoyer aux Professeurs de quelques jardins de botanique en Italie, les graines et plantes des espèces qui leur manquent.

$$
\text { Rapport }
$$

Le citn Bosc qui a été chargé d'emballer et expédier la collection de poissons pétrifiés offerte par la ville de Vérone, sollicite le remboursement de quelques dépenses faites à cette occasion, et qui ne devaient pas être imputées sur la somme qui lui a été accordée pour frais de voyage. La note jointe à sa lettre, porte le total de ces dépenses à la somme de $479 \mathrm{fr} .50 \mathrm{c}$., dont il a d'autant plus besoin qu'elle fait partie de celle de 600 fr. qu'il a emprunté à Vérone, et qu'il s'est engagé de payer à son retour à Paris.
Il ajoute qu'en sa qualité d'Inspecteur des pépinieres de Versailles, il a pris avec les Professeurs des jardins de botanique qu'il a visités pendant son séjour en Italie, l'engagement de leur faire quelques envois de graines ou de plantes des espèces qui leur manquent. Il prie le Mtre de l'autoriser à le remplir ; il lui transmettra la note de ses envois lorsqu'il seront effectués.

Je vous propose, Citn. Mtre, d'ordonner le payement de la somme réclamée. Vous voudrez bien prononcer en même tems sur la seconde demande du Citn Bosc.

Décision du ministre dans la marge du document :

Décis. [décision] du 10 Bre [Brumaire] an 12, appé [approuvé] une indemnité ou remboursement des six cent francs

Autorisé à expédier des graines

Remis le 25 Bre à la comptabil. [comptabilité]

\section{DOCUMENT 31 - 9 NOVEMBRE 1803}

Brouillon d'une Lettre de Jean-Antoine Chaptal, ministre de l'Intérieur, à Louis Augustin Guillaume Bosc (AN, F/17/1537, sous-dossier Gazola).

17 Bre [brumaire] an 12

Indemnité de 600 fr. accordée. Il est autorisé à envoyer Itatie les graines et plantes qu'il a promis aux Professeurs de quelques jardins de botanique en Italie.

$$
\text { Le Mtre [Ministre] }
$$

Au Citn Bosc, rue des maçons n 407

Je vous préviens, Citn, que je vous ai accordé une indemnité de 600 fr. pour acquitter les dépenses occasionnées par l'encaissement de la collection de poissons pétrifiés de Vérone. En vous adressant à la $4^{e}$ division du ministère le ler jeudi de nivose prochain, vous serez informé du jour où cette somme sera payée par le Trésor public.

D'après votre demande, je vous autorise à envoyer aux Professeurs des jardins de botanique que vous avez visités en Italie les graines et plantes que vous leur avez promis.

Je v. s. [vous salue] 


\section{DOCUMENT 32 - SANS DATE [VERS FIN 1803]}

Note anonyme d'un agent du ministère de l'intérieur (AN, F/17/1537, sous-dossier Gazola).

Affaire du C. Gazola

\section{Note}

Le C. Gazola offrit au premier consul de la part de la ville de Vérone une collection de poissons pétrifiés, la quelle fut acceptée par un arrêté spécial.

Le ministre ayant chargé le C. Bosc naturaliste de se transporter à Verone pour faire emballer et expédier cette collection, il se rendit à sa destination.

Avant son départ, le Cit. Gazola avait déclaré que la collection offerte par la ville de Vérone neétait point encore en sa possession, qu'elle nétait pas même formée, et que les pieces qui devaient la composer faisaient encore partie de ce quil avait de fossiles de cette nature dans sa maison. Il prétendait qu'il avait dessin d'être lui-même sur les lieux, pour désigner les pièces quil voudrait bien livrer au commissaire : mais en même temps il refusa sous des prétextes d'affaires de partir avec lui.

Cependant la municipalité de Véronne mit le C. Bosc en possession des pieces qui se trouvaient chez le C. Gazola; et elles furent emballées, lorsquil parût évident que l'on attendrait envain plus longtemps celui-ci mais au moment de l'expédition les caisses furent arretées à la douane par ordre spécial du vice president de la république italienne, qui déclara lui meme au commissaire qu'elles ne partiraient qu'après que le C. Gazola de retour aurait choisi celles des pieces qu'il destinait à former la collection acceptée.

D'un autre coté, le cit. Cazola se plaint quil est dépouillé, et demande que les caisses soient arretées partout où elles seront, et reportées chez lui, pour quil en sépare tout ce qui n'a pas du être livré.

Cette manière d'agir du C. Gazola est d'autant plus étrange que le catalogue des objets dont cette collection est composée, en va pas audelà de 191 articles, rapportés et figurés dans la piece ci jointe. D'après ce qu'en avait dit le citoyen qui d'ailleurs est propriétaire de la montagne de Vestena-nuova appellée par lui-même une mine inépuisable de ces fossiles, on avait lieu de penser que la collection présentée au premier consul devait être plus considérable.

\section{DOCUMENT 33 - 2 JANVIER 1804}

Lettre du comte Ferdinando Marescalchi (1754-1816), ministre des Relations extérieures de la République italienne, à JeanAntoine Chaptal (1756-1832), ministre de l'Intérieur. La lettre est accompagnée d'une copie conforme de la lettre des officiers de la municipalité de Vérone à Gazola (Annexe 1[doc. 24]) (AN, F/17/1537, sous-dossier Gazola).

Paris, le 2 janvier 1804 an $3^{e}$ [de la République italienne] Citoyen Ministre,
L'entretien que j'eus dernièrement avec vous touchant l'affaire relative à la collection de poissons fossiles du Cen Gazola de Vérone, m’a donné lieu de penser que tous les détails n'en avaient pas été mis bien exactement sous vos yeux. Persuadé en même tems [sic] que votre équité vous fait desirer d'en être pleinement instruit, je vais avoir l'honneur de vous les présenter.

Le Cen Gazola sétait occupé, pour ainsi dire, toute sa vie à former une collection de fossiles de toute espèce et particulièrement de poissons pétrifiés. Non seulement il avait fait lui-même des recherches pénibles et coûteuses, mais encore il avait acquis divers cabinets, pour raison desquels il s'était engagé à payer plusieurs rentes viagères sélevant ensemble à 180 sequins ou environ 2.160 tournois.

Cette collection était devenue aussi assez intéressante, quant à la partie des poissons, pour que les commissaires des arts, envoyés par le Gouvernement français en Italie, durant la dernière guerre, souhaitassent d'en enrichir le Muséum National d'Histoire Naturelle. Le Cen Gazola ne pouvait guère résister à ce désir : ses poissons furent donc céder, même sans aucune convention bien expresse, et aussitôt on les fit transporter à Paris.

Il était pourtant juste de l'indemniser, et c'est à quoi il fut pourvu par un arrêté que le Cen Haller prit le 26 thermidor an 5, sur la demande des commissaires eux-mêmes et qui fut d'ailleurs approuvé par le Premier Consul alors Général en chef de l'Armée d'talie.

Cet arrêté, après avoir énoncé un état fourni par le Cen Gazola et des dépenses quil avait faites et des obligations qu'il avait contracté pour la collection, détermina qu'en dédommagement on lui transporterait en toute propriété une prairie nommée de Prebiano, située dans la commune de Villafranca, et dont il était reconnu que le produit sélevait à 12.000 livres de Venise ou un peu plus de 6.300 tournois.

Sur cela néanmoins il devait être chargé de faire deux payements, l'un d'une rente de $400^{l t}$ [livres] au capital de 10.000"t, accordée à la Société Italienne de Vérone, l'autre d'une somme principale de 4000lt attribuée au Cen Cagnoli, astronome, du même pays.

Mais cet arrangement, qui paraissait équitable, ne pu avoir son exécution, parceque la commune de Villafranca s'y opposa. Cette prairie de Prebiano lui avait été autrefois concédée par le Gouvernement vénitien et, jalouse de conserver cette propriété, elle fit des réclamations auxquelles on pensa quil était juste d'avoir égard.

Il fallut donc pourvoir de quelque autre manière à l'indemnité du Cen Gazola. C'est pourquoi, dans une transaction passée le 8 vendémiaire an 6 entre les agents du Gouvernement français et l'administration de l'Etat du Mantouan, il fut dit que cette administration "séparerait sur les biens qui lui étaient céder une portion quelconque produisant 3.000lt [livres] de Milan pour être remise en toute propriété au Cen Gazola, à l'effet de l'indemniser du cabinet de poissons pétrifiés et autres objets quil avait cédés à la République française».

Comment et pour quels motifs cette indemnité d'abord fixé à un revenu de près de 6.000 tournois, fût-elle réduite à un fonds ne produisant que 3.000lt de Milan, c'est-à-dire, de plus 
de moitié ? c'est ce que j’ignore. Tout ce que l'on voit, c'est que le Cen Gazola, qui n'avait point été partie dans la transaction, loin d'être content de ce qu'elle lui attribuait, ne manqua pas de s'en plaindre, et qu'il n'a cessé de renouveller sa réclamation, jusqu'à ce qu'enfin le Gouvernement français ait bien voulu y faire droit, ainsi que le dirai tout-à-l'heure.

Cependant, son goût pour les collections de ce genre ne sétait point effacé. Il continua de faire des recherche et fouillant surtout dans une montagne appelée Vestena-nuova, dont il est propriétaire, et qui est comme une mine inépuisable de ces fossiles, il rassembla bientôt une immense quantité de pièces, dont plusieurs présentaient de nouvelles espèces qui ne se trouvaient pas dans sa première collection.

Lorsqu'il eut réuni un assez grand nombre de ces dernières, l'idée lui vint de les offrir au Gouvernement français pour qu'elles fussent jointes à celles qui avaient été transportées à Paris et placées dans le Muséum. En cela, il ne voulait pas seulement faire un hommage au Premier Consul: il se proposait encore un autre objet qui flattait son amour propre. C'était de completter, d'embellir une collection dont il était le premier auteur, et dont il pensait que l'honneur lui serait toujours attribué.

Mais certaines considérations le déterminaient à ne point faire lui-même immédiatement son offrande. La commune de Vérone, dont il est habitant, souhaitait de donner au Premier Consul quelque témoignage de son respect, de sa reconnaissance, et le Cen Gazola se plut à lui en fournir le moyen. Il pensa même que l'hommage qu'il voulait faire serait plus digne du Consul, en étant présenté par une commune, que s'il l'était par un particulier. Ainsi donc il commença par céder à la ville de Vérone les pièces qu'il voulait donner, et ce fut ensuite au nom de cette même ville qu'elles furent offertes au Premier Consul.

Les circonstances furent encore cause que cette offre fût faite par le Cen Gazola lui-même et par un de ses compatriotes, le Cen Pinali [15]. L'un et l'autre, en effet, durent venir à Paris, dans le mois de décembre 1802, en qualité de députés de leur commune, pour solliciter quelques faveurs particulières auprès du Premier Consul, Président de la République italienne, et l'une des commissions qu'on leur donna fut celle de le prier d'agréer l'hommage qu'on voulait lui faire.

Il eut effectivement la bonté de l'accepter, et alors il fut question des mesures à prendre pour l'éxécution. Sur cela, il parut d'abord nécessaire d'envoyer quelqu'un à Vérone pour recevoir le présent et avoir soin de le faire transporter en France. L'on pensa même qu'il conviendrait de choisir une personne qui connût la collection déjà placée au Muséum, et qui fût par conséquence en état de mieux juger de ce qui pourrait le plus contribuer à la completter et embellir. Mais il vous plut, Citoyen Ministre, de donner cette mission au Cen Bosc, et le Cen Gazola ne put que respecter votre choix.

Cependant, ce présent ne pouvait guère être livré, sans que le Cen Gazola fût lui-même à Vérone. C'est que, malgré la cession qu'il

15 Gaetano Pinali (13 août 1759, Vérone - 14 janvier 1846, Vérone) en avait faite à la commune, les pièces dont il s'agissait étaient toujours restées dans sa maison et que de plus elles s'y trouvaient mêlées, confondues dans l'immense collection de tous genres qu'il a formée, et même avec beaucoup d'autres morceaux de même nature rassemblés, soit pour son propre usage, soit dans l'objet d'en enrichir les cabinets de Pavie et de Bologne. Il ne manqua point aussi de le représenter au Cen Bosc, le priant de différer son départ, jusqu'à ce que lui et le Cen Pinali eussent terminé les affaires pour lesquelles ils étaient venus à Paris, affaires qui étaient de la plus haute importance pour leur patrie, et qui pouvaient les obliger de prolonger leur séjour en cette ville de quelques mois. Mais le Cen Bosc témoigna le désir de partir sur le champ, alléguant qu'il aimait mieux attendre en Italie le retour du Cen Gazola, dont il sentait bien que la présence était nécessaire.

À la vérité, il fit bien entendre encore qu'il ne pensait pas qu'il dît être question d'un triage à faire, parcequ'il croyait que tout ce que le Cen Gazola avait rassemblé devait indistinctement être considéré comme ayant été donné au Gouvernement français. Mais le Cen Gazola, n'imaginant point qu'on pût se méprendre sur ses intentions et le sens de l'offre faite au nom de la commune, n'attacha point, pour le moment, trop d'importance à ce discours, et ne crut point devoir former dès lors une contestation sérieuse à cet égard.

Le Cen Bosc partit donc, laissant à Paris le Cen Gazola qui dût même y rester beaucoup plus longtems qu'il n'avait d'abord pensé, et qui d'ailleurs profita de l'occasion pour renouveller [sic] auprès du Gouvernement français ses réclamations relatives à l'inexécution de l'arrêté du 26 thermidor an 5, c'est-à-dire, au complément de l'indemnité qui lui avait été promise, et dont on ne lui avait accordé qu'une partie dans la transaction du 8 vendémiaire an 6.

Comme vous ê̂tes vous-même la bonté, citoyen Ministre de vous occuper de cette affaire, il est inutile que je vous dise comment elle fut terminée. Je vous prierai seulement de vous rappeler qu'il n'était question que de cette première collection que le Cen Gazola avait cédée aux commissaires pour les arts envoyés en Italie par le Gouvernement français, et dont il avait été entendu que le prix lui serait payé. Car, quant aux pièces nouvellement données par lui à la commune de Vérone et offertes par celle-ci au Premier Consul, c'était un hommage purement gratuit, dont les Véronais pensaient qu'ils seraient trop payés, s'il était accepté comme une marque de leur respect et de leur reconnaissance.

Quoiqu'il en soit, vers la fin du mois d'août, le Cen Gazola reçut une lettre des officiers municipaux de son pays qui lui causa le plus grand étonnement. Elle lui apprenait que le Cen Bosc, à peine arrivé à Vérone, avait aussitôt demandé que tout ce que le Cen Gazola avait rassemblé de poissons fossiles lui fût livré sur le champ et qu'on recherchât même s'il ne s'en trouvait pas quelques pièces déposées chez d'autres particuliers : que sur cela on lui avait représenté qu'on ne pouvait ni justement ni décemment s'introduire dans la maison du citoyen pendant son absence et la violer, pour ainsi dire, afin de se saisir même d'un don qu'il avait fait : qu'on lui avait remontré encore que la commune n'avait pu offrir que ce que 
le Cen Gazola avait bien voulu lui céder, et que celui-ci n'avait jamais entendu faire le sacrifice de tout ce qu'il avait rassemblé, mais seulement de ce qui pouvait completter [sic], embellir la collection du Muséum, à laquelle il était peu utile d'ajouter des doubles, des triples, des quadruples exemplaires; que le Cen Bosc n'avait rien voulu entendre; qu'il avait dit que, s'il $y$ avait de doubles, de triples orc exemplaires, on saurait bien les placer, puisqu'ils pourraient être agréables aux frères du Premier Consul et qu'alléguant sur le tout vos ordres et votre nom, qu'on se ferait un devoir de respecter, il avait impérieusement exigé qu'on satisfît à sa réquisition; enfin que, quoique ces procédés eussent paru bien extraordinaires, on avait cru ne pouvoir sortir d'embarras qu'en faisant tout ce que le Cen Bosc avait voulu.

Vous jugez aisément, citoyen Ministre, de la peine que dît éprouvé le Cen Gazola en recevant ces nouvelles; en apprenant qu'on s'était emparé de tout le fruit de ses longs et pénibles travaux, en voyant qu'on avait renversé tout d'un coup les divers projets qu'il avait formés, tant pour les progrès de la science, que pour l'utilité des établissements publics de la République italienne. Aussi se hâta-t-il de recourir à la justice et aux bontés du Premier Consul, lequel voulut bien prêter une oreille favorable à ses plaintes.

Il savait bien effectivement quel était le véritable objet, le véritable sens de l'offre qui lui avait été faite, et qu'il avait daigné accepter. Il savait bien que le Cen Gazola n'avait entendu donner que les pièces qui présentaient des espèces nouvelles et qui completteraient la collection du Muséum, ou celles encore qui, formant de plus beaux exemplaires, contribueraient à l'embellir. Il ne pouvait approuver d'ailleurs qu'on êेt, en quelque sorte, ravi de force un présent que le Cen Gazola avait voulu faire. Il me chargea donc d'écrire aussitôt, de son ordre, soit pour qu'on ne laissât partir de Vérone aucun des objets livrés au Cen Bosc, soit pour que, s'ils étaient déjà en route, on les arrêtat partout où ils se trouvaient. C'est aussi ce qui fut exécuté à Milan où les caisses, que le Cen Bosc s'était hâté d'expédier avec une précipitation, même nuisible à la conservation des pièces, étaient déjà arrivées, lorsque mes lettres y parvinrent.

Au surplus, le Cen Gazola ne tarda point de partir de Paris où, en dernier lieu, il avait travaillé, avec un zèle infatigable, dans le Muséum, à mettre la collection en un meilleur ordre et à la disposer à recevoir ce qui devait y être ajouté. En passant à Milan, il reconnut les caisses qui y avaient été arrêtées, et probablement il les fit retourner à Vérone. Je dis probablement, car, en ce moment, je ne sais pas d'une manière bien précise ce qu'elles sont devenues.

Mais, quoiqu'il en soit, ce Citoyen m'a écrit plus d'une fois depuis son retour dans sa patrie et je n'ai pu que louer les sentiments qu'il m'a témoignés. Il m'a mandé effectivement que malgré tout ce qui s'était passé, il était toujours également disposé à effectuer, dans toute leur étendue, et la cession qu'il avait faite à la commune de Vérone, et l'offre que le Premier Consul avait daigné accepter ; qu'il se promettait de joindre à la collection du Muséum un grand nombre de pièces très intéressantes, soit par leur nouveauté, soit par leur belle conservation; qu'il espérait même pouvoir l'enrichir beaucoup plus qu'il ne s'en était d'abord flatté; et qu'enfin il n'oublierait rien pour donner au Gouvernement français, dans cette circonstance, des preuves certaines de son dévouement et de son respect.

Voilà, Citoyen Ministre, le récit de toute cette désagréable affaire. Je vois avec quelque peine qu'il est bien plus long que je ne l'avais compté en commençant; je ne chercherai pourtant point à l'abréger, car je pense que le désir d'être bien informé vous fera sacrifier volontiers quelques moments de plus. Mais d'ailleurs je m'abstiendrai de toute réflexion, et je laisserai à votre justice et à vos lumières de tirer toutes les conséquences auxquelles cet exposé peut donner lieu. Permettez-moi seulement d'y joindre la copie de la lettre écrite au Cen Gazola par les officiers municipaux de Vérone[16], et dont je vous ai parlé ci-dessus. Elle vous fera voir toujours plus comment on avait entendu et la cession et l'offre dont il s'agit, sur quoi je puis dire encore que moi-même, à qui le Cen Gazola avait été adressé par sa commune, et qui eut l'honneur de le présenter au Premier Consul, j'avais toujours également compris qu'il était question de donner, non tout ce qu'il avait rassemblé, mais seulement tout ce qui pouvait servir à completter, à embellir la collection du Muséum.

Agréez, Citoyen Ministre, l'assurance de ma haute considération. F. Marescalchi

\section{DOCUMENT 34 - 29 JANVIER 1804}

Lettre (numéro d'enregistrement 683) de François Mami, fondé de pouvoir de Gazola, à Jean-Antoine Chaptal, ministre de l'Intérieur (AN, F/17/1537, sous-dossier Gazola).

Paris le 8 Pluviose an 12 A son Excellence le Ministre de l'Intérieur Citoyen Ministre

Par arrêté du Gouvernement en date du 12 Germinal an 11 e, transmis par votre lettre du 23 du même mois et même année, le citoyen Gazola de Veronne a obtenu une gratification annuelle de 2500 francs, en acquis de ce qui lui restait dîu sur le prix de la collection de poissons petrifiés qu'il a cédés en l'an $5^{e}$ au Gouvernement.

N'ayant rien reçu depuis le 20 vendre [vendemiaire] an $12^{e}$, le citoyen Gazola prie votre Excellence de vouloir bien lui faire delivrer les ordonnances de payement de la $d$. gratifon [dite gratification] depuis cette époque et succéssivement.

J'ai l'honneur d'être avec respect

François Mami fondé de pouvoir

Rue Honoré $n^{\circ} 71$

Maison du notaire

16 Il s'agit d'une copie du document 24 (Annexe 1). 


\section{DOCUMENT 35 - 22 FÉVRIER 1804}

Rapport de Jean-Pierre Barbier-Neuville, chef de la 3e division du Ministère de l'Intérieur, à Jean-Antoine Chaptal, ministre de l'Intérieur accompagné d'une décision du ministre (AN, F/17/1537, sous-dossier Gazola).

\section{3e Division}

Bureau des Sciences

On prie le Ministre de prononcer sur la demande du Cen Gazola, tendant à obtenir le payement de sa pension.

Paris, le 2 ventose de l'an 12 de la République française Rapport présenté au Ministre de l'Intérieur

Le Cen Gazola de Vérone sollicite par l'entremise de son fondé de pouvoir, le payement de la pension de 2.500 francs accordée en acquit de ce qui restait dû sur le prix de la collection de poissons fossiles qu'il a vendue en l'an 5 au Gouvernement.

Le fondé de pouvoir fait observer qu'il n'a rien reçu depuis le 20 vendemiaire dernier, et il demande que le Ministre lui fasse délivrer les ordonnances à dater de cette époque, et successivement.

On ajoutera que, par une lettre qui fait l'objet d'un rapport particulier le Cen Fourcroy annonce que le Cen Gazola promet d'envoyer ce printems, les poissons de la collection offerte au Premier Consul, et qui manqueraient à celle que possède le Muséum d'histoire naturelle.

Le Ministre est prié de faire connaître sa décision.

$$
\text { Le Chef de la 3e Division }
$$
J.P.Barbier-Neuville

Note de Jean-Antoine Chaptal, ministre de l'Intérieur, écrite dans la marge du rapport :

Il ne sera donné suite à la demande du fondé de pouvoir du Cen Gazola que lorsque ce dernier aura rempli l'engagement qu'il a contracté et que la livraison de ses collections et de celle de la ville de Verone pour lesquelles le gouvernement lui avait annoncé une pension seront effectuées [sic]

$$
\text { Chaptal }
$$

\section{DOCUMENT 36 - 29 FÉVRIER 1804}

Copie d'une lettre de Jean-Antoine Chaptal, ministre de l'Intérieur, à François Mami, fondé de pouvoir de Gazola (AN, F/17/1537, sous-dossier Gazola)

\section{9 ventose an 12}

Il sera donné suite à la demande du Citn Gazola, quand il aura rempli ses engagemens.

Le Mrre au Citn Mamin rue St Honoré $n^{\circ} 71$ Maison du notaire. En qualité de fondé de pouvoir du Citn Gazola, vous m'invitez, Citn à faire acquitter les arrérages de la pension qui lui a été accordée pour terminer le payement de la collection qu'il a vendue en l'an 5 au Gouvernement.

Je vous préviens qu'il ne sera donné suite à une demande que lorsque le Citn Gazola aura rempli l'engagement qu'il a contracté, et que la collection de poissons fossiles, offerte par la ville de Vérone, sera effectuée.

$$
\text { J.v.s. [Je vous salue] }
$$

\section{DOCUMENT 37 - 20 OCTOBRE 1804}

Copie de l'arrêté Jean-Étienne-Marie Portalis, ministre de l'Intérieur par interim, suivi de la copie d'une lettre de Portis à Faujas de Saint-Fond, suivi enfin d'un ordre de paiement du Le payeur général de la trésorerie nationale en date du 22 ventôse an 13 (13 mars 1805) (ADI, J 547).

\section{Ministre de l'Intérieur}

Paris le 28 Vendre [vendémiaire] an 13

Le Ministre de l'Intérieur par interim, arrête

$1^{\circ}$. M. Faujas l'un des professeurs administrateurs du muséum d'histoire naturelle, est autorisé à faire annuellement, comme par le passé, des voyages pour l'intérêt et l'avantage de cet établissement.

$2^{\circ} \mathrm{M}$. Faujas voyagera cette année en Italie.

$3^{\circ}$ Conformément à l'arrêté du Directoire du 7 fructidor an 5, ses indemnités seront de 13000 francs sur laquelle somme il payera le dessinateur ou le secrétaire qu'il pourra emmener dans ses courses

$4^{\circ} \mathrm{M}$. Faujas est chargé de demander et d'expédier en France les poissons pétrifiés qui ont été offerts par la ville de Veronne et par M. Gazzola pour augmenter la collection du muséum d'histoire naturelle de Paris; à raison de cette mission particulière, les indemnités auxquelles $M$. Faujas aura droit d'après l'article précédent, seront augmentés de 3000 francs

5. Ces indemnités de 13000 et de $3000 f$ seront prises sur les fonds destinés au transport des objets de sciences et arts et payées en 4 payements de mois en mois. Le premier payement s'effectuera au mois avant l'époque que M Faujas indiquera pour son départ. Le Ministre de l'Intérieur par interim Signé Portalis

\section{Ministre de l'Intérieur \\ Paris le 28 Vendémiaire an 13}

Le Ministre de l'Intérieur par interim à M. Faujas professeur administrateur du Muséum d'histoire naturelle

Je vous transmets, Monsieur, une ampliation de l'arrêté par lequel j'autorise de nouveau les voyages que vous étiez dans l'usage d'entreprendre annuellement pour l'avantage du muséum d'histoire naturelle. J'ai senti combien une plus longue interruption serait préjudiciable à un établissement que vous enrichiriez d'objets rares et intéressants.

Vous voudrez bien, Monsieur, voyager cette année en Italie. J'espère de votre zêle et de votre esprit conciliateur, que vous pourrez obtenir la collection des poissons fossiles que la ville de Veronne avait offerte 
au Gouvernement, mais que nous ne possédons point encore. Quand vous aurez fixé l'époque de votre départ, je vous remettrai, des notes sur cette affaire et vous indiquerai la marche à suivre pour lever les obstacles qui s'opposent à l'expédition de ces poissons en France. Je vous salue, signé Portalis

"Pour copie conforme aux originaux des dites pièces demeurées jointes à une ordce [ordonnance] expédiée par le Ministre de l'Intérieur le huit ventose an treize [27 février 1805] sous le $N^{\circ}$ 754 exercice an 13 de la somme de 4000 et dont le payement a été fait par moi soussigné payeur général des dépenses diverses de la trésorerie nationale le vingt un ventose an treize"

$\grave{A}$ Paris le vingt deux ventose an treize

\section{DOCUMENT 38 - 2 NOVEMBRE 1804}

Lettre de Faujas de Saint-Fond au ministre de l'Intérieur (AN, F/17/1537, sous-dossier Faujas).

Paris, ce 11 brumaire an 13 À son Excellence le Ministre de l'Intérieur

M. Faujas Professeur administrateur du Museum d'histoire naturelle

Monseigneur,

J'ai reçu la lettre par la quelle Votre Excellence me prévient que par son arrêté du 8 Vendémiaire dernier elle autorise de nouveau les voyages que je suis chargé de faire chaque année, pour procurer au Museum et au Jardin des plantes les objets qui tendent à enrichir ce monument des Sciences naturelles; \& à reconnoitre en même tems nos richesses départementales sous le même point de vue.

Votre Excellence me charge aussi d'aller cette année en Italie pour réunir et faire expédier la riche collection que la ville de Vérone et $M r$ de Gazola ont offerts [sic] au gouvernement et qui a été acceptée par Sa Majesté l'Empereur. Comme j'ai fait mes dispositions pour me mettre en route avant que l'hiver arrive j'ai l'honneur de vous prier de vouloir me faire parvenir les notes nécessaires pour cet objet \& ordonnances en conformité de l'article 5 de votre arrêté le payement des fonds que vous avez fixé et qui doivent être mis à ma disposition pour survenir aux dépenses nécessaires pour cette mission.

Je suis avec respect de votre Excellence | Monseigneur | le très obeissant serviteur

Faujas

\section{DOCUMENT 39 - NOVEMBRE-DÉCEMBRE 1804}

Lettre ( $\mathrm{n}^{\circ}$ d'enregistrement 683) de Jean-Baptiste Nompère de Champagny, ministre de l'Intérieur, à Faujas de Saint-Fond (AN, F/17/1537, sous-dossier Faujas). Cette lettre ne fut pas envoyée, Faujas ayant retardé son départ au mois de juin 1805.
3 me Division

Bureau des Sciences et des Beaux Arts

Paris le [...] Frimaire, an 13 Le Ministre de l'Intérieur,

À Monsieur Faujas, l'un des professeurs administrateurs du Muséum d'histoire naturelle

Je vous préviens, Monsieur, que j'ai ordonné le payement de la somme de seize mille francs qui vous est accordée pour le voyage que vous devez faire cette année en Italie, suivant la décision de mon prédécesseur qui vous a été notifiée le 28 vendémiaire dernier. Cette somme sera acquittée par acomptes de quatre mille francs, de mois en mois, et aussitôt que l'état des fonds mis à ma disposition par le gouvernement le permettra. Le chef de la 4 me Division informera néanmoins de l'époque du premier payement et de celle des autres, à mesure qu'ils pourront avoir lieu.

Vous trouverez ci-joint quelques pièces concernant votre mission particulière à Véronne. Vous voudrez bien vous conformer aux dispositions que renferme celle qui est indiquée sous le $n^{\circ} 1$.

Je vous invite à prévenir $M$. Gazzola que du moment où j'aurai la certitude qu'aucun obstacle ne s'oppose à ce que le Gouvernement français soit mis en possession de la collection de poissons pétrifiés qu'il lui a offerte, je ferai rétablir, s'il y a lieu, la pension de quinze cents francs ${ }^{17}$ qui lui a été accordées le 12 Germinal an 11, et dont le payement est suspendu depuis le 1er Brumaire an 12 [24 octobre 1803], à cause du retard apportée dans la délivrance des objets promis.

J'ai l'honneur de vous saluer

Note dans la marge d'une autre main

La somme a été payée. Paris le 9 floreal an 13 [29 avril 1805]. Cette lettre n'a pas été expédiée, M. Faujas ayant retardé l'époque de son voyage.

\section{$N^{\circ} 1$}

3me Division

Bureau des Sciences et des Beaux Arts

Paris le [...] Frimaire, an 13

Instruction du Ministre de l'Intérieur pour servir à M. Faujas dans sa mission à Véronne

M. Faujas, à son arrivée à Véronne, fera reconnoître les titres de sa mission par les autorités de la ville; et, après leur avoir remis copie de l'arrêté portant acceptation du don offert en leur nom au Gouvernement Français par M. Gazzola, il s'occupera de constater, par un inventaire, le nombre, l'état de conservation et la variété des objets qui composent la collection. Il enverra un double de cet inventaire au Ministre de l'Intérieur. Il contractera tous les marchés nécessaires pour l'encaissement des objets, et choisira pour leur transport, la voie et les moyens qui lui paroîtront les plus propres à en assurer la conservation. Il enverra au Ministre des doubles de ces marchés, pour que le payement s'excutent aux époques qui y seront déterminées.

Le ministre de l'Intérieur

17 C'est une erreur, il s'agit de 2500 francs. 
$N^{\circ} 2$ [copie de l'extrait des registres des délibérations du gouvernement du 12 germinal an 11, arrêtant l'acceptation du don (voir Annexe 1[doc. 12])]

\section{DOCUMENT 40 - 11 JANVIER 1806}

Lettre de Cyprien Prosper Brard aux administrateurs du Muséum (AN, AJ/15/596, séance du 15 janvier 1806).

Paris 11 janvier 1806

Messieurs les administrateurs

J'ai reçu le 10 janvier une lettre de Mr votre collègue Faujas. Il mécrit de Vicence en date du 31 décembre, ce même jour il a du partir pour Padoüe, où il séjournera peu de jours; ensuite il profitera de l'évacuation des troupes ennemies pour se rendre à Venise delà, il ira à Milan où les caisses de la collection de Vesténanova l'attendent pour passer en France; il doit y arriver dans un mois à compter du 31 décembre.

Les lettres ou les paquets que l'on désirerait lui faire parvenir doivent être adressés : Chez Monsieur l'Abbé Charles Amoretti naturaliste à Milan pour remettre à $M$. Faujas.

J'ai cru, Messieurs, que ces détails pouvaient vous interesser, c'est pourquoi je me suis empressé de vous les communiquer.

J'ai l'honneur d'être avec le plus profond respect | Messieurs, | votre très humble et très obéissant serr [serviteur] | Brard

\section{DOCUMENT 41 - 16 AVRIL 1806}

Lettre de Barthélémy Faujas de Saint-Fond à Jean-Baptiste Nompère de Champagny, ministre de l'Intérieur (AN, F/17/3979, sous-dossier 13, Mission de Faujas de Saint-Fond en Italie).

\section{A Milan le 16 avril 1806}

A son excellence le ministre de l'intérieur

Faujas St Fond l'un des professeurs administrateur au muséum d'histoire naturelle

\section{Monseigneur}

mon illustre ami, $M$. Delacépede [sic], a pûu vous instruire dans le tems des divers voyages que je fais depuis plus de huit mois que je suis en Italie relativement aux sciences et aux arts; ne voulant pas dérober vos momens à l'utilité publique, j'attendois d'avoir quelques résultats à vous mander, et comme j'avois poussé mes excursions jusqu'au fond de la Carinthie, pour y suivre quelques objets d'arts de la plus haute importance, je me suis souvent éloigné des routes de postes, que les lettres que j'aurois pî̀ écrire alors, ne seroient certainement point parvenues à leur destination.

Le premier objet que j'avois à remplir en entrant en Italie, étoit de me rendre à Vérone, pour y recevoir la collection des poissons fossiles destinés par la ville de Verone et par M. de Gazola pour sa majesté l'empereur, pour faire le complément des rares objets que le muséum d'histoire naturelle possède en ce genre; je remplis cette mission du premier abord, sous le feu du canon de l'ennemi au moment même du passage de l'Adige ; j'expédiai l'envoi pour Milan en prennant toutes les surettés [sic] possibles. Il devoit attendre mon retour dans cette dernière ville, où je voulois réunir les divers minéraux que je pourrois recueillir dans les montagnes de la haute Italie et du Tyrol, ainsi que dans celle de la Stirie [Styrie] et de la Carinthie, afin qu'au terme de mon voyage tout pu partir en même tems par la voie la plus assurée et la plus économique. Tout est en règle dans le moment. Les caisses sont toutes réunies ici et prettes [sic] à partir. Elles n'attendent plus qu'une autorisation afin de n'être point visitées et être exemptes de droits à leur entrée en France. Car elles courroient de grands risques à présent qu'elles sont embalées avec le plus de soin possible, sil falloit les déclarer pour les visiter. J'ai obtenu avec facilité à Milan qu'elles iroient jusqu'aux frontières sans être visitées; je pars demain pour Turin afin de me concerter avec le général Menou [18], afin d'obtenir la même facilité à leur entrée en France par Verceil ; j'aurai l'honneur de vous mander de Turin ce que j'aurai fait à ce sujet. Messieurs Sorési commissaires banquiers, qui se chargent ordinairement de tous les envois en France, même pour son A. I. [Altesse Impériale] le vice roi [19], et qui ont la confiance publique, se chargeront d'expédier le tout par la voie des roulliers jusqu'à Lyon, et de Lyon à Paris par d'autres roulliers que leur fournissent leurs correspondants ; ils recommanderont très particulièrement toutes les caisses afin qu'on les place et qu'on les manie avec ménagement. Le prix sera le prix courant du commerce. Son A. I. le vice roi m'auroit fait donner avec plaisir un grand caisson militaire qui auroit rempli le même objet avec plus de certitude encore, et il avoit même eu la bonté de donner à ce sujet ses ordres au général Charpentier [20], chef de son état major, qui les avoit notifié au commissaire ordonnateur Joubert [21] ; mais tous les chevaux et tous les caissons militaires, ont filés depuis quelque tems vers l'armée de la Dalmatie; nous avons été contraint par là, d'employer un autre moyen. C'est celui des frères Sorési commissionnaires.

Je me réserve d'avoir l'honneur de metre [sic] sous vos ieux [sic] lorsque je serai rendu à Paris, Monseigneur, les résultats de mes voyages, et les succès que j'ai été assez heureux d'obtenir non seulement pour procurer pour la collection du muséum des objets rares que j'ai receuilli [sic] de mes mains dans les hautes montagnes du Tyrol, de la Carinthie, du Frioul, de l'état vénitien orc: et qui font partie de l'envoi ; mais j'ai obtenu des résultats non moins satisfaisants, sur divers objets d'arts

18 Jacques-François de Menou, baron de Boussay, (3 septembre 1750, Boussay, Indre-et-Loire - 13 août 1810, Venise).

19 Eugène Rose de Beauharnais (3 septembre 1781, Paris 21 février 1824, Munich), fils adoptif de l'empereur Napoléon Ier, avait la qualification d'Altesse impériale, vice-roi d'Italie. 20 Henri François Marie Charpentier (1769-1831), chef d'état-major de l'armée française en Italie.

21 Louis Joubert (3 novembre 1762, Le Mans - $1^{\text {er }}$ janvier 1812, Rosno, Russie). 
et manufactures, qui nous manquaient, ou sur lesquels nous

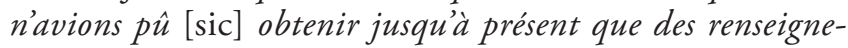
ments vagues ou incertains. J'aurai l'honneur de me présenter à votre excellence, les preuves et les faits à la main à ce sujet. Je suis avec respect |Monseigneur |votre très humble | et très obéissant serviteur | Faujas St Fond

\section{DOCUMENT 42 - 23 AVRIL 1806}

Lettre de Barthélémy Faujas de Saint-Fond à Jean-Baptiste Nompère de Champagny, ministre de l'Intérieur (AN, F/17/3979, sous-dossier 13, Mission de Faujas de SaintFond en Italie).

A Turin le 23 avril 1806

À son excellence le ministre de l'intérieur

Faujas St Fond, l'un des professeurs administrateur au muséum d'histoire naturelle Monseigneur

en passant au Bureau des douanes de Verceil, j'ai pris les renseignements nécessaires relativement à l'entrée des vingt trois caisses destinées pour le muséum d'histoire naturelle que j'ai laissées à Milan entre les mains de Messieurs les frères Sorési, banquiers expeditionnaires à Milan qui les expedieront pour Paris aussitôt qu'ils seront informés que les dittes caisses ne seront point ouvertes au Bureau de Verceil, et seront exemptes en même tems de droits. Il seroit donc necessaire d'obtenir de $M^{r}$ Colin [Collin] cette exemption de visite et de droits pour vingt trois caisses grandes et petites ayant pour subscription muséum d'histoire naturelle à Paris et faire aviser Messieurs Sorési Banquiers expéditionnaires à Milan, qu'ils peuvent faire expedier lorsque la dispense de visite sera obtenue. J'ai l'honneur de prévenir votre excellence, que si l'on en agissoit autrement, on exposeroit des objets très précieux pour les sciences et qui ont donné beaucoup de peines à emballer avec tout le soin et toutes les precautions possibles, à être détruits.

Je passerai dans quelques jours le Mont Cenis pour me rendre, par la voie de Grenoble, dans ma possession que j'ai, à Saint-Fond département de la Drôme, où je me délasserai pendant une quinzaine de jours de repos, des fatigues d'un voyage penible, qui dure depuis plus de huit mois, aussitôt que je serai un peu remis, je me rendrai à Paris, et j'aurai l'honneur de metre [sic] sous vos ieux [sic], le rapport de mon voyage, non seulement relativement à l'histoire naturelle, mais encore aux arts utiles, et à quelques branches d'industrie qui peuvent interesser l'empire français.

Je suis avec respect | Monseigneur | De votre excellence, le très humble et très obéissant serviteur

Faujas-St Fond

\section{DOCUMENT 43 - 23 AVRIL 1806}

Lettre du général Jacques-François de Menou à Jean-Baptiste Nompère de Champagny, ministre de l'Intérieur (AN, F/17/3979, sous-dossier 13, Mission de Faujas de Saint-Fond en Italie).

Turin 23 avril 1806 Le gal Menou, command gal $^{\text {Soc }}$ \&o

A son Excellence Monseigneur de Champagny, Ministre de l'Intérieur, Grand cordon de la Légion d'Honneur Monseigneur,

J'ai l'honneur de prévenir, Votre Excellence, que l'illustre savant Mr Faujas de St Fond, est passé hier à Turin. Il m'a prié de donner mes soins au passage très prompt de vingt trois caisses, renfermant les résultats précieux de ses pénibles \& savantes recherches dans plusieurs provinces de l'Italie, dans le Frioul, la Styrie, la Carinthie, la Cargniole [Carniole] orc orc. Parmi ces différents objets se trouve la précieuse collection de poissons pétrifiés, dont la ville de Vérone, fait hommage à sa Majesté l'Empereur \& Roi.

Mr Faujas de St Fond, m'a exprimé toute l'importance \& l'intérêt que mettoit le gouvernement à la prompte arrivée à Paris des 23 caisses. Il ne m'a pas laissé ignorer combien, Votre Excellence, désiroit que les caisses arrivassent à Paris avant le 20 Mai \& aussi qu'elles ne fussent point ouvertes en passant à Verceil. J'ai l'honneur de prévenir, Votre Excellence, que j'ai invité Mr le Préfet du Dépt de la Sésial22] \& Mr Souiris Directeur des Douanes, à ne point faire ouvrir les caisses, mais à les faire plomber pour remplir le vou de la loi relativement aux douanes. J'ai pensé, Monseigneur, que cette mesure rempliroit à la fois toutes les intentions du Gouvernement.

Je suis avec respect, | Monseigneur, | De Votre Excellence |Le très humble \& très obéissant serviteur

Menou

\section{DOCUMENT 44-28 AVRIL 1806}

Lettre de Jean-Baptiste Nompère de Champagny, ministre de l'Intérieur, à Jean-Baptiste Collin de Sussy, directeur général des Douanes (AN, F/17/3979, sous-dossier 13, Mission de Faujas de Saint-Fond en Italie).

28 avril 1806

A Monsieur le Conseiller d'Etat Collin, Directeur général des Douanes

Monsieur le Conseiller d'Etat, Mr Faujas St Fond, Professeur au Musée d'Histoire naturelle, est occupé depuis 8 mois à former, pour cet établissement, soit en Italie, soit dans les contrées environnantes, des collections de productions des divers genres. Il me mande de Milan, que les caisses qui les renferment sont arrivées à la frontière. L'ouverture et la visite de ces caisses dans lesquelles les objets ont été emballés avec le plus grand soin pourrait [sic] leur être extrêmement

22 Ancien département français entre 1802 et 1814, dont Verceil était le chef-lieu, et qui était frontalier avec le Royaume d'Italie. 
préjudiciable pour la suite du voyage. Je vous prie donc Monsieur le Conseiller d'Etat de donner les ordres nécessaires, pour que ce précieux dépôt puisse arriver intact jusqu'à l'établissement public auquel il est destiné, et de l'exempter à cet effet, des formalités ordinaires.

Recevez, Monsieur le Conseiller d'Etat, l'assurance de mon sincère attachement

Champagny

\section{DOCUMENT 45-28 AVRIL 1806}

Lettre de Jean-Baptiste Nompère de Champagny, ministre de l'Intérieur, au général Jacques-François de Menou (AN, F/17/3979, sous-dossier 13, Mission de Faujas de Saint-Fond en Italie).

28 avril 1806

A Monsieur le Général Menou, Commandant général des Départements au delà des Alpes

Monsieur le Commandant général, j'avais écrit moi-même à Monsieur le Conseiller d'Etat Directeur générl des Douanes, pour le prier d'exempter des formalités ordinaires, à l'entrée du territoire français, les 23 caisses renfermant les collections formées par Mr Faujas St Fond, et destinées au Musée d'histoire naturelle. J'approuve donc en ce qui me concerne l'ordre donné au Préfet de la Séria et au Directeur de la Douane de Verceil, pour la libre entrée de ces caisses, et je vous remercie d'avoir concouru à les faire arriver en France sans derangement et sans obstacles.

Recevez, Monsieur le Commandant général, l'assurance de ma considération la plus distinguée

Champagny

\section{DOCUMENT 46 - 17 MAI 1806}

Lettre de Jean-Baptiste Collin de Sussy, directeur général des Douanes aux administrateurs du Muséum d'histoire naturelle (AN, AJ/15/596, séance du 21 mai 1806).

Paris, le 17 mai an 1806 Le Conseiller d'Etat, Commandant de la Légion d'honneur, Directeur général des Douanes de l'Empire,

A Messieurs les Administrateurs du Muséum d'histoire naturelle

Messieurs, vous demandez qu'on ne visite point à la frontière plusieurs caisses renfermant des objets relatifs à l'histoire naturelle, que $M^{r}$ Faujas a été chargé de recueillir à Véronne, et d'autres villes d'Italie, pour le Muséum.

Sur l'invitation qui m'en a été faite par le Ministre de l'Intérieur, j'ai donné l'ordre à Verceil de les expédier, sous plomb et par acquit à caution, pour la Douane de Paris. Je présume que cet envoi est le même que celui dont vous mentretenez; s'il en était autrement; je vous prie de me le faire connaître.

J'ai l'honneur de vous saluez

Collin

\section{DOCUMENT 47 - 10 JUIN 1806}

Lettre de Barthélémy Faujas de Saint-Fond à Bernard-Germain Lacépède (AN, AJ/15/596, séance du 18 juin 1806). A St Fond par Loriol département de la Drôme, le 10 juin 1806
Mon cher et illustre ami

Je suis arrivé ici ily a environ un mois assez bien portant malgré les fatigues d'un long et penible voyage; mais la traversée du Mont Cenis que je fis à pied depuis Suze [Suse] jusqu'à la Novalèze [Novalaise], me fit beaucoup de mal à la vue, lorsque je fus dans les nèges [sic] qui couvroient entièrement le sommet et tout le plateau de cette grande montagne; comme ily avait ce jour la [sic] un soleil très vif qui se reflettoit sur le blanc, ma vue en fut affectée, d'une manière peu sensible d'abord, mais le soir une rougeur me couvrit toute la face, j'avais comme du sable dans les ieux [sic], et le lendemain j'éprouvai une chaleur brulante et comme hérésipelateuse [érésipélateuse]; j'avois eu tord [sic] de ne pas suivre l'exemple des ouvriers qui étoient occuppés à déblayer les nèges qui avoient couverts le chemin la veille, ils avoient tous une gaze noire sur les ieux, ce qui m'étonna pour des hommes de peine accoutumés à vivre au milieu des nèges; mais ils me dirent avec raison; qu'un beau soleil à cette élévation, qui ne se montre à la vérité que rarement sans nuage, étoit une chose d'autant plus dangereuse, qu'on ne s'en appercevoit pas d'abord, que sa chaleur paraissoit au contraire très agréable, mais qu'il en résultoit des inflamations, quelque fois si grave, particulièrement lorsque le soleil prennoit de la force, que plusieurs curieux avoient entièrement perdu la vue; qu'une gaze noire étoit un préservatif assuré, je savois cela, et j'en avois autrefois fait usage, en voyage avec Guettard dans les Hautes Alpes; au mois de juillet et d'août dans la région où la nège ne fond jamais, mais je ne crû [sic] pas que dans une traversée qui ne dure que huit heures, et où je ne ne croyoit pas trouvers autant de nège alors, il me fallut prendre tant de précaution; enfin je fus obligé de maretter [sic] quelques jours à Grenoble. L'inflamation de la peau se termina par de vives démengeaisons, et par la chute de la partie superficiele [sic] de l'epiderme; mais les ieux sans avoir l'apparence très malade, avoient reçu une atteinte qui ne me permettoit ni de lire, ni d'écrire, ni de supporter le trop grand jour; j'ai pris quelques bains, je me suis lavé les ieux très souvent avec de l'eau froide sans vouloir suivre d'autre remede, et enfin je commence à écrire depuis quelques jours et l'inflamation se calme sensiblement. Je proffite avec plaisir et empressement de ce bon moment, pour vous remercier de la lettre aimable que vous avez eu la bonté de m'écrire et que j'ai trouvée ici en arrivant; sans la circonstance dont je viens de vous donner les trop longs détails, jaurois été empressé de répondre à votre lettre, et d'avoir l'honneur de vous faire part ainsi qu'à mes chers collègues, de toutes les dispositions qui avoient été faites pour l'envoi que j'avois receuilli [sic], ou achetté dans le Tyrol, la Styrie et la Carinthie, pour ma propre collection, que mon intention est neant moins [sic] de partager avec le muséum pour les objets qui peuvent lui manquer.

L'on vient de mécrire d'Italie que tout l'envoi qui avoit été expédié de Milan devoit être arrivé à Paris. J'avois vîu [sic] à 
Turin le général Menou, et je lui avois fait part du désir que javois que les caisses ne fussent point ouvertes au Bureau des douanes de Verceil particulièrement celles qui renfermoient les poissons fossiles, qui sont très fragiles, surtout lorsqu'on décloute les caisses; je lui recommandai très particulièrement de vouloir les faire examiner avec soin lorsqu'elles passeroient à Turin, en le prévenant quelles [sic] ne tarderoient pas à partir, et quelles [sic] n'attendoient que l'ordre de $M^{r}$ Colin [Collin], que le ministre de l'intérieur devoit avoir obtenu; non seulement le général Menou s'empressa de reprendre mes vues, mais il me dit qu'ayant le commendement [sic] général de son département, il alloit écrire au directeur de la douane de Verceil, pour faire passer l'envoi sans ouvrir les caisses, à les visiter à Paris au muséum lorsquelles [sic] y seroient arrivées; or comme je compte quelles doivent être dans ce moment, et que je ne pourrai me metre [sic] en route pour Paris que le 1er du mois prochain [juillet 1806] à cause de l'incommodité de mes ieux, que la vivacité du jour fatigue encore trop. Il est à propos que j'aie l'honneur de vous faire part de quelques observations relatives à l'envoi, et qui seront utiles dans le cas où le désir de voir les collections de Gazola, ne permettrait pas d'attendre mon arrivée; je sens très bien qu'il est bon d'en faire jouir promptement les savans et le public, surtout si l'on a un lieu favorable pour les placer.

$1^{\circ}$ chaque caisse de la collection envoyée de Vérone, et arrangé avec tout le soin possible devant moi contient une feuille qui désigne le nombre des objets qu'elle renferme. J'en ai conservé un duplicata afin de les collationner.

$2^{\circ}$ lorsque Mr de Gazola reçu de Milan l'envoi qui avoit été fait par Bosc, en vertu d'un arretté [sic] du ministre de l'intérieur $M^{r}$ Chaptal et dont Mr Marescalci [Marescalchi] sollicita et obtint le rapport, $M r$ de Gazola qui étoit à cette époque à Paris, craignant que cet envoi n'eut souffert en route on fit vérifier l'état en présence d'un membre du gouvernement italique. Il en résultat qu'une des plus grandes caisses qui renfermoit le poisson le plus volumineux, se trouva avoir souffert, et ce poisson éprouva une cassure transversale. Ce n'étoit certainement pas la faute de Bosc, mais une aussi grande caisse étant difficile à manier, et les roulliers étant très peu soigneux, ils la déchargèrent des charettes sans ménagement. Mr de Gazola fit constater le fait par un procès verbal et Mr l'abbé Ammoretti son chargé de procuration. Laissé en dépôt à Milan cette grande caisse, avec une autre aussi grande qu'il suppose avoir eu le même sort. J'ai tout vérifié moy-même à Milan cette avarie. Personne n'étant en état de réparer dans cette ville l'accident arrivé à ce bel yctiolite, j'ai consulté plusieurs personnes, et j'ai prefféré d'envoyer le poisson tel qu'il étoit mais en l'assujetissant avec des câlles, des étoupes et en prennant toutes les précautions afin que le mal n'augmente pas; comme la caisse qui renferme ce grand individu n'avoit pas la solidité requise, jai fait faire une double caisse, chose indispensable, à cause du passage du Mont Cenis où les mulets peuvent s'abatre et tout briser. Vous trouverez donc, ce grand poisson, tel qu'il existoit à Milan dans le dépôt où il étoit lorsqu'on me la remis. S'il n'a pas souffert d'avantage, on pourra le réparer facilement à Paris. Je devois vous instruire de ce fait. $3^{\circ}$ parmis [sic] les caisses qui furent renvoyées dans le tems de Milan à Mr de Gazola, il s'en trouva une particulière appartenant personnellement à $M^{r}$ Bosc, qui a ce que m'a dit Mr de Gazola renferme des objets d'histoire naturelle en insectes et minéraux que le naturaliste avoit receuilli dans son voyage en Italie. Cette caisse est quarrée, peut peser environ quatre vingt dix ou cent livres, et porte pour subscription, à son excellence le ministre de l'intérieur; il m'a paru juste et convenable de la faire expédier, pour quelle fut rendue à son propriétaire $M^{r}$ Bosc qu'on aura la bonté de faire prévenir et qui viendra la reconnoitre.

4. toutes les autres portent pour subscription muséum d'histoire naturelle

5. $M^{r}$ le sénateur Abrial[23] me pria à Milan, chez le vice roi, de permettre qu'une caisse de livres qu'il vouloit envoyer en France, acompagna l'expédition du muséum et afin qu'elle fut respectée en route et non visitée à l'entrée de France. Il fit écrire dessus muséum d'histoire naturelle avec les deux lettres suivantes DF autant que je puis m'en rappeller [c'est-à-dire Desfontaines] [24]. Mr Abrial me dit qu'il écriroit à notre collègue Desfontaines son ami pour réclamer cette caisse, or comme elle est pesante, il me dit qu'il rembourseroit le port.

6. toutes les caisses de la collection de Gazola renfermant des poissons de Bolca, avec l'inventaire par-dessus sont destinées pour le muséum et portent l'inscription muséum d'histoire naturelle sans petites lettres. Les caisses qui ont la même adresse muséum d'histoire naturelle, avec deux petites lettres B.F. sont à moy. Les unes renferment des livres d'histoire naturelle dont plusieurs nouveaux qui manquoient à ma bibliothèque, que j'ai achetté à Venise, à Padoue, à Milan et à Pavie; il y en a plusieurs caisses. Les autres caisses toujours avec les lettres B.F. renferment la collection volcanique du Vicentin, des monts Euganéens et des fossiles que j'ai receuilli [sic] dans le Plaisantin, dans le Veronnois et ailleurs, et les minéraux que j'ai ramassé dans le Tyrol et la Carinthie. Je rembourserai les frais de port de ces caisses à moy appartenantes, et je partagerai avec le muséum tout ce qu'il y a de double, ayant eu attention de receuillir tout ce que j'ai pî en double. S'il s'y trouve quelque chose d'unique qui manque au muséum, je lui en ferai avec grand plaisir homage. Je suis trop fatigié dans ce moment, après avoir fini cette lettre, d'en écrire une seconde pour l'administration. Mais comme elle ne seroit dans le fond qu'une répétition de celle cy, j'espère que vous aurez la complaisance de faire part ce que j'ai l'honneur de vous écrire à l'assemblée le plutôt que vos moments vous le permetront.

Je désire bien ardament [sic] d'avoir le plaisir de me réunir à eux. J'ai receuilli [sic] quelques faits nouveaux dans les diverses parties des sciences qui les occupent, et je serai bien empressé de les communiquer à chacun d'eux, et de leur renouveller les assurances de mes plus tendres sentiments

Faujas

23 André Joseph, comte Abrial (19 mars 1750, Annonay 13 novembre 1828 , Paris).

24 René Desfontaines. 


\section{DOCUMENT 48 - 3 NOVEMBRE 1806}

Lettre de Faujas de Saint-Fond, à Jean-Baptiste Nompère de Champagny, ministre de l'Intérieur (AN, F/17/1537, sousdossier Faujas).

Au jardin des plantes le 3 novembre 1806

Son Excellence le ministre de l'intérieur de l'empire français

Faujas St Fond, professeur de géologie l'un des administrateurs du muséum d'histoire naturelle

Monseigneur

Je partis pour l'Italie en vertu de la commission spéciale qui me fut donnée, pour aller recevoir de la ville de Véronne, la belle collection des poissons fossiles qui avoit été offerte à sa majesté l'empereur des français roi d'Italie, qui avoit bien voulu l'accepter.

J'arrivai à Verone lorsque l'armée du maréchal Masséna étoit en présence de celle du prince Charles, et n'en étoit séparée que par l'Adige; je m'exposai plus d'une fois pour sauver cette collection, et la garantir des bombes et des obus que le prince Charles lançoit sur la ville de Verone, lorsque notre armée le força à la retraite, et passa l'Adige. Je mis tous les soins possibles à inventorier, faire emballer et à expédier cette collection pour Milan, ce qui se fit avec tous le succès possible. Comme j'avois à receuillir [sic] pour le museum, les productions minéralogiques qui manquoient à nos collections, et qui se trouvoient dans le Tyrol, je dirigeai ma marche sur ces hautes montagnes. Je poussai jusqu'en Carinthie, parce que j'avois à y voir des manufactures de la plus haute importance, qui manquent à la France. Son Alt. impériale le vice roi de Milan, fit part à Munich, à sa majesté l'empereur Napoléon de l'objet de ce voyage, qui fut pénible et même dangereux, et en tout le succès désiré.

Je me rendis ensuite à Venise, lorque son Alt. impériale vint en prendre possession. Son Altesse impériale m’invita à visiter les manufactures du paj̈s venitien, et majourna à Milan, où je fus rendu le douze mars 1806.

Ainsi à cette époque, javois terminé le voyage de 1805 et anticipé de deux mois et douze jours sur celui de l'an 1806.

À Milan, son Altesse impériale, ne me laissa pas oisif. Je fis établir à sa demande, une manufacture de la plus haute importance, qui est en pleine activité. Voyez la lettre de son excellence, le ministre de l'intérieur du royaume d'Italie.

Je partis de Millan [sic] à la fin du mois de mai, j'ai l'honneur de vous prier de prendre lecture de la lettre que son altesse impériale le vice roi d'Italie voulu bien m'écrire lui-même, la veille de mon départ. Lorsque jeu l'avantage d'aller prendre congé de son Altesse.

J'ai donc voyagé cinq mois, non compris le retour, accompagné de mon dessinateur, de deux domestiques, cette année 1806, et j'en ai fait l'avance, sur les fonds que je croiois pouvoir toucher sur ceux de 1806 destinés à mes voyages; ces fonds me sont donc bien légitimement dûs. Je me proposois d'aller faire encore et sans demander d'autres fonds que ceux de cette année, un voyage, à Gênes, pour faire le complément de mon voyage d'Italie, que je me propose de publier.
J'ose espérer de la justice de votre excellence, Monseigneur, qu'elle aura la bonté de prendre en considération une demande appuiée sur des titres irrécusables, et que la vie pénible que je mène dans mes voyages, les dangers que je cours, l'avantage qu'en retire [sic] le muséum et les arts peuvent me mériter quelque reconnaissance.

Je suis très respectueusement $\mid$ de votre excellence le très humble et très obéissant serviteur

Faujas

\section{DOCUMENT 49-25 NOVEMBRE 1806}

Lettre des professeurs administrateurs du Muséum d'histoire naturelle, cosignée par René Desfontaines (1750-1833) et René Just Haüy (1743-1822), à Jean-Baptiste Nompère de Champagny, ministre de l'Intérieur (AN, F/17/1537, sousdossier Faujas).

\section{Muséum d'histoire naturelle}

Les Professeurs administrateurs du Muséum d'histoire naturelle à son Excellence le Ministre de l'Intérieur,

\section{Monseigneur,}

Nous avons reçu la lettre par laquelle Votre Excellence nous fait l'honneur de nous consulter relativement au voyage que $M^{r}$ Faujas de St Fond a fait en Italie pendant les années 1805 et 1806. Elle nous demande si ce voyage doit être considéré comme équivalent à deux de ceux qu’il est chargé de faire annuellement. Les professeurs du Museum après avoir examiné la question que vous leur proposer se sont décidés pour l'affirmative, et ils croyent devoir vous exposer succintement [sic] les motifs de leur opinion.

Mr Faujas est chargé par Votre Excellence de faire chaque année pour la minéralogie un voyage dans l'intérieur de la France; ce voyage est de 3 à 5 mois. Celui qu'il vient de faire a eu lieu dans un paÿs qui étoit le théatre de la guerre et a duré 13 mois. Il partit de Paris au mois de juin 1805, pour faire transporter en France la collection de poissons fossiles que la ville de Verone et M. de Gazola avoient offerte à sa majesté l'Empereur et Roi; et il arriva à Verone lorsque l'armée française et celle du Prince Charles étoient en présence sous les murs de cette ville. La collection fut conservée par ses soins et il l'a fit partir pour Milan d'où elle est arrivée au Muséum dans le meilleur état. Cette mission remplie, il alla à Venise pour examiner une riche collection de poissons de l'Adriatique qu'il étoit important de comparer aux fossiles du Mont Bolca déposés au Museum. Il resta à Venise jusqu'au 15 janvier et ne pouvant revenir à cette époque parce que le passage du Mont Cenis étoit fermé par les neiges, il écrivit que se trouvant plus voisin du Tyrol que de Milan, il alloit en visiter les montagnes et pousser son voyage jusqu'en Styrie et en Carynthie [Carinthie], pays riche en minéraux et où se trouvent des fabriques et des usines qui manquent à la France. Il écrivit de Klageafurth [Klagenfurt] et annonça qu'il avoit receuilli des objets précieux pour le Museum. 
Arrivé à Padoue au mois de mars, il nous annonça qu'il partait pour Milan, où il fut retenu quelque tems par le Vice Roi. C'est de cette ville que furent expédiées les collections.

Mr Faujas de retour à Paris au mois de juillet s'est occupé de faire son cours de géologie, et de mettre en ordre les productions qu'il avoit rapporté [sic].

D'après les faits exposés ci dessus on voit que ce naturaliste ayant rempli sa mission et se trouvant forcé de différer son départ à cause de la difficulté du passage des montagnes au mois de janvier, il a pris un parti avantageux pour l'histoire naturelle en visitant la Styrie et la Carynthie d'où il a rapporté beaucoup d'observations et des objets précieux.

Il est encore certain que cette prolongation de voyage a du lui causer une dépense d'autant plus considérable qu'il avoit avec lui un dessinateur.

Il nous a assuré qu'il avoit été obligé d'emprunter de l'argent pour subvenir à des frais qu'il n'avoit pu prévoir.

Nous pensons d'après cela, Monseigneur, qu'il est de votre justice d'indemniser Mr Faujas d'une double dépense puisqu'en prolongeant son voyage il a rendu des services importans aux Arts et à l'histoire naturelle.

Nous avons l'honneur de présenter | à Votre Excellence, | Monseigneur, | nos hommages respectueux

Desfontaines, dteur [directeur] Haüy | secrétaire

\section{DOCUMENT 50 - 31 DÉCEMBRE 1806}

Lettre des professeurs administrateurs du Muséum d'histoire naturelle, cosignée par René Desfontaines et René Just Haüy, à Jean-Baptiste Nompère de Champagny, ministre de l'Intérieur (AN, F/17/1537, sous-dossier Faujas) (une copie de cette lettre est également présente aux Archives départementales de l'Isère, dossier J 547).

\section{Muséum d'histoire naturelle}

Paris $31 X^{\text {bre }} 1806$

Les Professeurs administrateurs du Muséum d'histoire naturelle à son Excellence Le Ministre de l'Intérieur

Monseigneur,

Nous avons reçu la nouvelle lettre par laquelle Votre Excellence nous demande un rapport sur les résultats et l'utilité des voyages que Mr Faujas est chargé de faire annuellement et notamment à l'égard du dernier qu'il a fait en Italie: Votre Excellence nous invite aussi à lui faire savoir si le cours de géologie de l'an 13 a eu lieu.

L'assemblée après avoir délibéré mûrement sur les différens objets contenus dans la lettre de Votre Excellence, croit devoir s'en référer, par rapport au premier, à l'arrêté rendu par S. E. le Ministre des Cultes[25], dans le tems où il exerçoit par intérim les fonctions du Ministre de l'Intérieur.

25 Jean-Étienne-Marie Portalis, voir Annexe 1[doc. 37].
Nous joignons ici une copie de cet arrêté. À l'égard du dernier voyage fait en Italie, Votre Excellence verra dans la lettre que nous avons eu l'honneur de lui adresser en date du 25 novembre dernier, qu'il avoit en particulier pour objet d'obtenir pour la France et d'y faire transporter la collection des poissons pétrifiés de Verone. Nous avons eu soin d'annoncer dans le tems à Votre Excellence que cette collection est effectivement arrivée et a été déposée dans le Muséum.

Nous lui avons également rendu compte dans notre lettre du 25 novembre des résultats de ce voyage.

C'est la précipitation qu'il a fallu mettre à cette commission particulière, à cause de la guerre dont on étoit menacé, qui a empêché, Mr Faujas de faire son cours de l'an 13, et il nous a assuré que cette année il avoit fait un plus grand nombre de leçons que le règlement ne lui en prescrit.

Nous devons particulièrement rappeler à Votre Excellence, l'article de l'arrêté ci-joint qui décide que la somme affectée aux voyages de $M^{r}$ Faujas ne sera point prise sur les fonds $d u$ Muséum ; vous savez parfaitement, Monseigneur, qu'ils sont à peine suffisant pour les objets actuels.

Nous avons l'honneur de présenter | à Votre Excellence, | Monseigneur, | nos hommages respectueux

Desfontaines, dteur [directeur]

Haüy | secrétaire

\section{DOCUMENT 51 - 10 JANVIER 1807}

Lettre du ministre de l'Intérieur, Jean-Baptiste Nompère de Champagny, à Faujas de Saint-Fond (ADI, J 547). Une copie de cette lettre est également présente aux Archives nationales (F/17/1537, sous-dossier Faujas).

Paris, le 10 janvier 1807 Le Ministre de l'Intérieur,

À Monsieur Faujas de St Fond, professeur au muséum d'histoire naturelle.

J'ai décidé, Monsieur, que sur l'exercice de 1806, il vous serait payé une somme de dix mille francs pour acquitter l'excédent de frais du voyage que vous avez fait en Italie, l'année précédente.

Vous serez instruit par la comptabilité de mon Ministère de l'époque où vous pourrez recevoir cette somme au trésor public. Je vous préviendrai au surplus, Monsieur, que faute de fonds, il me sera impossible à l'avenir d'autoriser de pareils voyages. J'ai l'honneur de vous saluer

Champagny

\section{DOCUMENT 52 - 17 AOÛT 1808}

Lettre du comte Ferdinando Marescalchi, ministre des Relations extérieures du Royaume d'Italie, à l'Empereur Napoléon Bonaparte (AN AF/IV/959, document 299). 
M. Gazola, de Verone, réclame le payement d'une pension, de 2.400 qu'il a obtenue en compensation d'un riche cabinet d'histoire naturelle cédé au Gouvernement français.

Rapport à sa Majesté l'Empereur des Français, Roi d'Italie Sire,

M. J. B. Gazola, de Vérone, ayant cédé au Gouvernement Français un très riche et très précieux Cabinet d'histoire naturelle, Votre Majesté daigna, par décret du 12 Germinal an 11, lui accorder en compensation une pension de 2.400f [26].

Quelques mal-entendus sétant élevés au sujet de la consignation des objets qui composaient ce Cabinet, M. Chaptal, alors Ministre de l'Intérieur, supposant qu'il n'était point livré, suspendit le payement de la pension de M. Gazola.

M. Gazola privé de sa pension en réclame le payement de la justice et de la munificence de Votre Majesté.

Daignez, Sire, agréer l'hommage du profond respect avec lequel je suis | de Votre Majesté

Paris, le 17 Août 1808

le très soumis, très dévoué et très fidèle serviteur \& sujet F. Marescalchi

\section{DOCUMENT 53-16 SEPTEMBRE 1808}

Lettre des professeurs administrateurs du Muséum d'histoire naturelle, cosignée par Georges Cuvier et Louis-Nicolas Vauquelin, à Emmanuel Crétet, comte de Champmol, ministre de l'Intérieur (en réponse à une demande du ministre écrite le 13 septembre 1808 suite à une réclamation de Gazola) (AN, F/17/1537, sous-dossier Gazola).

Paris le 16 septembre 1808 Les Professeurs Administrateurs du Muséum d'histoire naturelle A son Excellence Le Ministre de l'Intérieur Comte de l'Empire

\section{Monseigneur}

Votre Excellence, nous annonce que Mr Gazola de Véronne réclame le payement d'une pension qui lui a été accordée pour une collection qui a dî̀ nous être remise, et nous demande si cette remise a eu lieu et quelle peut être la valeur de cette collection.

Voici les renseignements qu'il nous est possible d'offrir à Votre Excellence.

Il ya au muséum deux collections de poissons pétrifiés tirées du Mont Bolca, près de Veronne par les soins de Mr Gazola \& arrivées à deux époques différentes.

La première fut acquise et donnée en présent au Muséum par S.M.I. [Sa Majesté Impériale] à la suite de ses premières campagnes d'Italie. Il parait que M.M. Bertholet et Thouin, alors commissaires pour les sciences près l'armée d'Italie proposèrent cette acquisition au général en chef, mais nous ignorons absolument quelles conditions furent accordées à $M^{r}$ Gazola et $M^{r}$ Thouin

26 C'est une erreur, il s'agit en fait de 2500 francs. l'un de nous n'a pas pu nous en instruire. Elle arriva vers la fin de l'an VI, dans 13 caisses.

La seconde collection est arrivée plus recemment, il parait qu'elle avait été offerte à S.M.I. par la ville de Veronne. $M^{r}$ Chaptal, l'un des prédécesseurs de Votre Excellence, ainsi qu'il nous l'écrivit le 19 Prairial an XI avait envoyé Mr Bosc pour la chercher; mais ce savant ayant éprouvé quelques difficultés, Mr Portalis ministre par interim, envoya par arrêté du 28 vendémiaire en XIII, Mr Faujas l'un de nous pour cet objet et $M^{r}$ Faujas expédia en effet cette collection qui est arrivée en bon état l'été suivant [27].

Nous ignorons encore si la ville de Véronne fit un marché avec Mr Gazola ờ à quelles conditions celui-ci livra cette deuxième collection. Ce qui est certain, c'est que l'une \& l'autre sont magnifiques. La première qui remplit une des salles de notre muséum, en fait un des plus beaux ornemens; la seconde qui n'est pas encore exposée faute de place, mais qui le sera bientôt, graces [sic] aux augmentations d'édifices obtenues par la bienveillance de Votre Excellence, est moins nombreuse, mais elle consiste en pieces plus volumineuses.

Il est cependant difficile d'en apprécier la valeur en numéraire; Votre Excellence sait que ces sortes de choses n'ont pas de prix courants ; mais la peine qu'il a fallu pour retirer d'une carriere, des objets aussi nombreux, et pour les conserver aussi bien, tout délicats qu'ils sont, a dîu exiger de grands frais, et si la pension de 2400 francs[24] dont parle $M^{r}$ Gazola est le prix des deux acquisitions faites par le Gouvernement comme elle ne représente qu'une somme de 2400 francs, elle est certainement inférieure à la valeur des objets : peut être même une seule des deux collections pourrait-elle être estimée à ce taux; mais pour oser l'affirmer à Votre Excellence, il faudrait connaitre le prix de la main d'auvre à Véronne \& le plus au moins d'abondance des poissons dans la carriere, deux circonstances dont nous ne sommes pas instruits.

Nous avons l'honneur de présenter $\mid$ A Votre Excellence |Monseigneur | Nos hommages respectueux.

G. Cuvier $d^{r}$ [directeur] Vauquelin secre [secrétaire]

\section{DOCUMENT 54-19 SEPTEMBRE 1808}

Lettre du comte Ferdinando Marescalchi, ministre des Relations extérieures du Royaume d'Italie, à Emmanuel Crétet, comte de Champmol, ministre de l'Intérieur (lettre reçue le 8 octobre 1808) (AN, F/17/1537, sous-dossier Gazola).

S.E.M. [Son Excellence Monsieur] le Comte de l'Empire Cretet, Ministre de l'Intérieur

Monsieur le Comte,

27 C'est une erreur, la collection arriva à Paris vers le mois de juin 1806, soit près de deux ans après l'arrêté du 28 vendémiaire an 13 (20 octobre 1804). 
Votre Excellence m'annonce par la lettre qu'elle m'a fait l'honneur de m'écrire le 13 de ce mois que d'après les ordres de S.M. elle se dispose à lui faire un rapport sur l'affaire de $M$. Gazola de Vérone.

Je lui demanderai la permission, puisqu'elle s'occupe de cet objet de lui rappeler succinctement quelques faits qui y ont rapport, \& qui remontent à l'adminiton [administration] de ses prédécesseurs.

Le Gouvernement français à l'époque des premières campagnes d'Italie ayant desiré avoir une collection de poissons et plantes fossiles pétrifiés que M. Gazola avait formée à Vérone, elle lui fut cédée et transportée presqu'aussitôt à Paris, où on la plaça au Musée d'Histoire naturelle.

Il était juste d'indemniser M. Gazola, d'autant plus qu'il avait lui-même dépensé beaucoup d'argent, \& avait même contracté des dettes pour completter of enrichir ce cabinet.

Aussi, sur la proposition des commissaires des arts, l'administrateur des finances prit un arrêté approuvé par le Général en chef, qui transportait en toute propriété à $M$. Gazola la prairie de Prebiano, dont le revenu était estimé 6.300 tournois.

Des difficultés de toute espèce qu'il serait trop long de détailler ici empêchèrent M. Gazola de jouir jamais de cette propriété.

Une transaction du 8 Vendre [Vendemiaire] an 6 entre les agents français o l'adon du Mantouan résuisit la rente de $M$. Gazola, malgré sa vive réclamation, à 3.000 livres de Milan, qui répondent à $2.302 f 55^{c}$.

M. Gazola ayant continué à faire faire des fouilles dans la montagne de Vestena-Nuova, dont il est propriétaire, et qui est comme une mine inépuisable de pétrifications, recueillit peu à peu une assez grande quantité de pièces nouvelles, qui jointes à la première collection, pouvaient y ajouter un très grand prix. Aussi conçut-il le projet de les offrir à S.M. l'Empereur et Roi, alors Premier Consul, \& dans cette vue, il se résolu à les céder à la ville de Véronne, pour qu'elle put les présenter en son nom. Cette ville ayant, à cette époque quelques graces à demander, envoya à Paris M. Gazola lui-même, de qui S.M. animée du désir d'augmenter et d'embellir la collection du Musée, daigna agréer l'hommage qui lui fut offert de ce précieux supplément.

M. Bosc, naturaliste, fut envoyé à Vérone par le Ministre Chaptal pour assister au choix des pièces qui pouvaient convenir au Musée de Paris, et veiller à leur transport. Mais, arrivé à Vérone, il éleva une prétention singulière; ce fut qu'on devait lui livrer la collection entière de M. Gazola, quelque fut l'état de désordre où elle était, et sans attendre le retour du propriétaire, qui pourtant l'avait exigé. Des caisses furent remplies de tous ces objets ramassés indistinctement chez M. Gazola, et dirigées sur Paris. S.M. informée de la manière dont M. Bosc avait rempli sa mission, ordonna que les caisses seraient arrêtées partout où elles se trouveraient et remises à la disposition de M. Gazola.

Cependant $M$. Gazola continuait de solliciter le règlement de sa première affaire; car il était bien constant que son premier cabinet était d'une grande valeur, lui avait été acheté, \& qu'aucun des arrangements pris avec lui n'avait eu son exécution. Enfin un
Décret du 12 Germinal an 10[28] lui accorda en compensation une pension de $2.400 f$ [24].

M. Chaptal supposant d'après tous les malentendus qui avaient eu lieu, \& d'après ce qui s’était passé au sujet de la mission de $M$. Bosc, que le complément offert au nom de la ville de Vérone par M. Gazola n'avait pas été livré, suspendit le payement de cette pension.

M. Gazola administre la preuve que depuis 2 ans $M$. le professeur Faujas St. Fond a reçu le complément de la collection, \& s'est chargé de le transporter à Paris. Il réclame d'après cela le payement de sa pension, \& je ne vois pas en effet ce qui pourrait s'y opposer.

Agréer, je vous prie, Monsieur le Comte, l'assurance de ma haute considération.

Le ministre des Relat. Ext. du Royaume d'Italie Paris, le 19 septembre 1808.

\section{F. Marescalchi}

\section{DOCUMENT 55 - 21 SEPTEMBRE 1808}

Rapport du ministre de l'Intérieur, Emmanuel Crétet, comte de Champmol, à l'empereur Napoléon Ier (AN, AF/IV/959, document 298). Un brouillon de ce rapport est également conservé dans le dossier AF/17/1537, sous-dossier Gazola.

Compte rendu des obstacles et incidents qui ont suspendus jusqu’à ce jour le payement à Mr Gazola, de la gratification annuelle qui lui a été accordée pour la collection de poissons et plantes fossiles des environs de Veronne, cédée au Museum d'Histoire naturelle.

Les mêmes motifs n'existant plus, il va être pourvu au payement de l'année courante; il sera pourvu ensuite à celui de l'arriéré autant que le permettra la situation des fonds du ministère.

Paris, le 21 septembre 1808

Rapport à sa Majesté l'Empereur et Roi, Protecteur de la Confédération du Rhin

Sire,

Votre majesté a daigné me renvoyer un rapport de son Ministre des Relations extérieures du Royaume d'Italie, dont l'objet est de lever les obstacles qui s'opposent au payement de la pension de Mr Gazola de Veronne [sic], pension obtenue en dédommagement d'une collection de poissons et plantes fossiles par lui cédée à la France.

Elle m'a ordonné de lui faire un rapport sur cette affaire.

Les informations que j'ai prises auprès du Museum d'Histoire naturelle mont donné l'assurance que vers la fin de l'an 6, une collection précieuse dans ce genre était parvenue dans treize caisses, et qu'un second choix de poissons fossiles et de plantes était arrivé au Cabinet dans l'été de l'an 13, par les soins de Mr Faujas-St Fond, envoyé à cet effet, à Veronne, le 28 vendémiaire de la même année.

28 C'est une erreur, ce décret fut signé en l'an 11. 
Le premier envoi remplit une des salles du Museum et fait un des plus beaux ornements de cet établissement. Le second moindre en nombre, mais composé de pièces plus volumineuses, n'est point encore exposé. Il le sera aussitôt après l'agrandissement de la Galerie dont on s'occupe en ce moment, grace [sic] à la munificence de Votre Majesté. Ce dernier est un présent de la ville de Veronne, que Votre Majesté a daigné agréer.

Il est difficile d'apprécier en numéraire la valeur d'objets qui n'ont pas de prix courant dans le commerce; mais les frais d'extraction et ceux de conservation ont dî être considérables; l'administration du Museum estime que la gratification annuelle de deux mille cinq cents francs accordée par décret du $12 \mathrm{Ger}$ minal an XI, à $M^{r}$ Gazola, serait plustôt [sic] un prix insuffisant, qu'une récompense supérieure au mérite de ces précieuses pétrifications.

Je vais entrer dans quelque détails sur les difficultés et les obstacles que Mr Gazola a rencontrés jusqu'ici dans la jouissance de ce qui lui était dî.

Il devait d'abord avoir en toute propriété les prairies de Prebiano, d'un revenu de six mille trois cent livres tournois.

Il parait n'en avoir rien touché. Une transaction du 8 vendémiaire an 6 entre les agents français et l'administration du Mantouan reduisit la rente à deux mille trois cent deux francs cinquante cinq centimes. Ce second arrangement n'eut point son exécution[29].

$M r$ Gazola reclamait [sic] vivement et cependant continuait toujours les fouilles; il en ceda [sic] le nouveau produit à la ville de Veronne [sic] qui fit hommage de ce supplément à Votre Majesté, en députant vers elle l'auteur même de ces précieuses recherches.

Enfin, pour dédommager Mr Gazola des contrariétés qu'il avait éprouvées jusqu'à ce jour et lui tenir lieu d'une somme de 86.500 fr. qu'il reclamait comme lui restant due sur la vente de la première collection, Votre majesté, par un décret du 12 germinal an XI, lui accorda une gratification de deux mille cinq cent francs.

De nouveaux incidents dont il fut victime retardèrent l'arrivée à Paris du supplément offert par la ville de Veronne [sic] et motivèrent mal à propos la suspension du payement de la pension. Mais il ne peut plus y avoir aucune espèce de doute aujourd'hui sur la légitimité des reclamations soumises à Votre Majesté par son Ministre des Relations extérieures du Royaume d'Italie.

Il résulte de tous ces faits que la gratification accordée à $M^{r}$ Gazola doit lui être payée, et comme elle est assignée sur les fonds du Ministère de l'Intérieur, je vais prendre les mesures que me permettra la situation de ces fonds et commencer par le payement de l'année courante.

J'ai l'honneur d'être avec respect, Sire, de votre Majesté, de Votre Majesté Impériale \& Royale, le très humble, très dévoué et très fidèle serviteur et sujet.

Cretet

29 Il faut préciser que Gazola tirait bien une pension annuelle de ce second arrangement, même s'il la jugeait insuffisante.

\section{DOCUMENT 56 - 28 SEPTEMBRE 1808}

Brouillon d'une lettre d'un agent du ministère de l'Intérieur (probablement Jean-Pierre Barbier-Neuville) à Ferdinando Marescalchi (AN, F/17/1537, sous-dossier Gazola).

287 bre 1808

Monsieur, sur le rapport que j'ai présenté à S. M. l'Empereur \& Roi il a été décidé que le payement de la gratification annuelle de 2500 francs accordées à $M^{r}$ Gazola, était de nouveau autorisé. Gepayent auration autes fonds du Ministere pourront le permettre car it convient de remarquer que ce n'est point une pension quil a obtenue par le décret du 12 germinal an 11. Ee Ministre, parcedécret, a seutement été autorisé à lui assigner une gratification annuette.

Néanmoins En conséquence, je vais faire les dispositions prendre les mesures nécessaires pour faire compter sans délais l'année courante et l'arriéré à Mr Gazola. Il sera porté sur les états du ministère pour l'avenir; mais quant à l'arriéré de cette gratification je ne puis que promettre d'aviser aux moyens d'y faire face siten existe encore quetques uns à ma disposition.

Je me félicite, Monsieur, d'avoir fait quelque chose qui vous était agréable en faisant rendant justice à Mr Gazola.

Je prie Votre Excellence d'agréer l'assurance de ma haute considération.

Paris, le 26287 bre 1808

S.Ex. Mr de Marescalchi, ministre des Relations extérieures du Royme d'Italie

\section{DOCUMENT 57 - 18 OCTOBRE 1808}

Rapport de Jean-Pierre Barbier-Neuville, chef de la $3^{\mathrm{e}}$ division du Ministère de l'Intérieur, en date du 18 octobre 1808, à Emmanuel Crétet, comte de Champmol, ministre de l'Intérieur (AN, F/17/1537, sous-dossier Gazola).

Proposition d'accorder à M. Gazzola une gratification de trois mille cent quatre vingt quatorze francs, trente-trois centimes, qui lui tiendra lieu de sa pension pour l'an 14 (1806)

Paris, le 18 octobre 1808

Rapport présenté au Ministre de l'Intérieur, Comte de l'Empire.

Monseigneur,

D’après les Ordres de Sa Majesté, Votre Excellence a déjà pris des mesures pour faire acquitter la pension accordée à M. Gazzola, de Veronne, dont le payement étoit suspendu depuis le 1er Brumaire an 12. Mais à cet égard votre comptabilité ne peut satisfaire à ce que vous avez prescrit pour l'an 14 (1806) parcequ'il ne reste aucun fonds disponible pour les dépenses qui peuvent appartenir à cet exercice. Je me suis entendu avec le Chef de la 4 e Division, et nous avons reconnu que, pour remplir entièrement les intentions de l'Empereur envers M. Gazzola, il étoit nécessaire de lui faire compter une gratification équivalente à sa pension, et qui lui en 
tint lieu pour les quize mois et dix jours de l'an 14 ; (1806) la quelle gratification pouvoit se prélever sur le crédit affecté cette année aux secours et pensions en faveur des gens de lettres et artistes.

Le bordereau ci-joint fera connaître à Votre Excellence la possibilité de cette mesure. À la vérité le crédit se trouvera tellement épuisé qu'il ne vous permettra plus d'offrir à personne de nouveaux secours, mais vous aurez satisfait à une dette ancienne qu'il paroit impossible d'éteindre par tout autre moyen.

Je vous propose, Monseigneur, d'adopter les vues de ce rapport, et d'écrire à M. Mareschalchi [sic] pour le prier d'inviter M. Gazzola à charger quelqu'un à Paris de toucher les sommes qui lui sont dues.

\section{Le Chef de la 3 e Division J.P.B. Neuville}

Décision du ministre dans la marge du document :

Pour être paié sur 1809, les fonds de 1808 étant épuisés Le présent rapport devient sans objet au moyen de l'ordonnancement sur 1806

\section{DOCUMENT 58 - 2 NOVEMBRE 1808}

Brouillon d'une lettre d'Emmanuel Crétet, ministre de l'Intérieur, au comte Ferdinando Marescalchi (enregistré sous le numéro 272), accompagné du brouillon du rapport précédent (Annexe 1[doc. 57]) (AN, F/17/1537, sous-dossier Gazola).

Prière d'inviter M. Gazzola de nommer un fondé de pouvoir à Paris

Paris le 2 novembre 1808

Le Ministre de l'Intérieur à Monsieur Mareschalchi [sic], ministre des relations extérieures du royaume d'Italie

Monsieur, j'ai l'honneur de prévenir Votre Excellence que jallais presider les mesures nécessaires pour faire compter à $M$. Gazzola, les sommes qui lui sont dues pour la pension dont sa Majesté l'Empereur la [sic] gratifié par décret du 12 Germinal an 11. Il conviendrait que ce savant eut un fondé de pouvoir pour toucher à Paris ce qui lui revient et qu'il me fit connaitre la personne qu'il aura investie de sa confiance. Je vous prie, Monsieur, de l'engager à prendre cette précaution.

Je prie V. E. d'agréer l'assurance de ma haute considération

\section{DOCUMENT 59- 7 DÉCEMBRE 1808}

Lettre (numéro d'enregistrement 136) de Gazola à Emmanuel Crétet, ministre de l'Intérieur (AN, F/17/1537, sous-dossier Gazola).

\section{Excellence}

S.E. [Son Excellence] Mr Marescalchi a eu la bonté de me donner avis que Sa Majesté sur ma demande et le rapport favorable de V.E. [Votre Excellence] a daigné retablir ma pension de $2500 f$ a titre de gratification annuelle, et que V.E. a pris les mesures convenables pour me faire payer l'année courante et l'arriéré des années precedentes, desirant connaitre mon fondé de procuration qui doit toucher en mon nom les sommes courantes et celles qui sont dues. Je dois à V.E. les plus respectueux remerciements pour l'interêt qu'elle a daigné prendre a la decision d'une affaire si honnorable et si utile pour moi, et en vous priant d'en agreer l'hommage, j'ai l'honneur de dir [sic] a V.E. que j'ai nommé et je nomme a cet effet S.E. Marescalchi que ses bontés pour moi ont porté a ajouter ce nouveau titre a ma reconnoissance.

Mon zele pour la service de S.M. et mon goût pour les sciences me servent de motif pour offrir à V.E. mes soins, si par hazard [sic] elle le jugeait utiles, pour la classification des nouvelles pétrifications qui ne sont pas encore déterminées, ayant eu l'honneur de faire les premiers arrangements dans le Museum du jardin des plantes.

Je prie V.E. d'agreer l'hommage du profond respect avec le quel je suis

De votre Excellence

Véronne 7. Xbre [décembre] 1808

Le très humble et très obeissant serviteur Jean Baptiste Gazola

Note écrite d'une autre main dans la marge de la lettre :

M. In Bte [Jean Baptiste] Gazola témoigne à S.Ex. [Son Excellence] toute la reconnaissance dont il est pénétré pour le rapport favorable quelle à donner à S.M. [Sa Majesté], à l'effet de faire rétablir sa pension. Il annonce que $M$. Marescalchi a bien voulu se charger de la recevoir.

\section{DOCUMENT 60 - [22 DÉCEMBRE 1814]}

Lettre (enregistrée sous le numéro 13682) de Gazola au roi Louis XVIII, en date du 22 décembre 1814 d'après le Annexe 1[doc. 62] (AN, F/17/1537, sous-dossier Gazola).

\section{Sire}

L'epoque la plus fortunée de ma vie, celle où le plus auguste des Princes et le meilleur des Rois a honnoré de sa présence ma modeste habitation à Verone n'a pas été tout bonnheur pour moi. Mon attachement à la Famille Royale de France m'a rendu l'objet d'un odieuse persécution. Je fus incarcéré au moment de l'entrée de l'armée Française à Verone; le parc de ma maison fut abbattu [sic] et ma riche collection de poissons pétrifiés me fut enlevée, et transportée à Paris, ôu elle existe au Musée du Jardin du Roi.

Mr le comte Bertholet, pair de France, alors commissaire des arts, reclama contre cette injuste opération, et fit en sorte que le général en chef au nom du gouvernement Français ordonna qu'il me serait donné une compensation pour mon cabinet. Un fond fut en effet décrété pour mon indemnité, mais ce décret n'eut jamais d'exécution. Sur mes instances il me fut accordé un autre 
fond, et une pension viagère contre la quelle je reclamais, comme n'équivalant pas à la premiere assignation et à la valeur réelle de mon cabinet, pension qui même vient de mêtre suspendue.

Je supplie Votre Majesté de daigner ordonner la liquidation de cette affaire.

Je mets à Vos pieds Sire l'hommage du profond respect avec le quel je suis

De Votre Majesté

\section{DOCUMENT 61 - 12 JANVIER 1815}

Rapport d'une main anonyme probablement destiné au ministre de l'Intérieur (AN, F/17/1537, sous-dossier Gazola).

En juin 1797, lorsque par les soins de Mr le Cte Bertholet, alors commissaire des arts en Italie, le Gouvernt français fit prendre chez Mr le Cte Gazola de Vérone la collection de poissons pétrifiés dont il s'agit, elle fut estimée et le prix arrêté à une valeur dont le rapport annuel calculé à 4 p \% devait être de $6.000 \mathrm{f}$. de France environ. Mr Gazola a tacitement consenti cette fixation; en conséquence il lui fut assigné en aout 1797, en toute propriété des prairies dont le revenu était reconnu monter à peu près à cette somme. Mais ces prairies, situées dans l'arrondissem ${ }^{t}$ de Villafranca, non loin de Verone, fesaient partie de divers biens communaux et lorsque Mr Gazola voulut en prendre possession il fut assailli par les habitants des lieux circonvoisins, qui, le traitant de spoliateur, le contraignirent à se retirer. Il reclama et le Gouvernemt lui assigna ; en 9bre [novembre] 1797 [30], en échange de cette propriété une rente de 3.000 livres de Milan (environ 2.300 f. de France) sur la ville de Mantoue. Cette indemnité étant loin de compenser sa perte il réclama de nouveau et l'on créa indépendammt de la rente précitée (en avril 1803) en sa faveur, une pension viagère, sur le trésor de France, de 2.500f par an. Ces deux sommes ensemble n'égalant pas encore le prix arrêté primitivem ${ }^{t}$ par les autorités commises à cet effet, et d'ailleurs la pension viagère, telle qu'elle a été établie, ne pouvant dans ce cas suppléer au produit d'une propriété, Mr Gazola a fait, en personne de nouvelles observations au Ministre, mais elles n'ont pas été accueillies.

Le reclamant voyant l'inutilité de ses plaintes a différé de les réitérer espérant toujours qu'il se présenterait un tems [sic] plus favorable; ce tems est enfin arrivé et il le saisit avec d'autant plus d'empressement qu'il croit pouvoir tout attendre de la justice de Louis XVIII, Prince qui dans les tems d'un malheureux exil a daigné habiter pendant une année entière le propre château de Mr le Cete Gazola à Vérone.

Ce dernier demande donc que l'on considère comme non avenu tout ce qui a été fait, relativement à l'indemnité de sa collection de poissons pétrifiés, depuis la fixation primitive et que partant de ce point on liquide sa créance definitivement soit au moyen

30 C'est une erreur, c'est le 29 septembre 1797 que fut conclu cet arrangement (Fig. 6). d'un payemt total en espèces, ou par la cession d'une propriété d'une valeur relative à ses droits et dont il puisse disposer à son gré.

Si le Gouvernemt trouve plus convenable d'acquitter sa dette envers Mr Gazola par une rente sur l'état, le réclamant demande qu'elle soit perpetuelle et non viagère.

S. M. Louis XVIII en accueillant dernierement, dans une audience privée, Mr le Cte Gazola avec les témoignages les plus flatteurs d'une particulière bienveillance, l'a verbalement assuré que cette affaire serait promptemt terminée et à son entière satisfaction.

12 janvr 1815

Mr Gazola demeure rue Jacob, hôtel de Hambourg

\section{DOCUMENT 62-13 [JANVIER] 1815}

Lettre de Gazola à l'abbé de Montesquiou[31], ministre de l'Intérieur (AN, F/17/1537, sous-dossier Gazola).

\section{À son Excellence le Ministre de l'Intérieur Monseigneur}

Le 22 Decembre dernier il est parvenu dans les bureaux de $V$. E. une réclamation, en mon nom, relative au pris [prix] d'une collection de poissons pétrifiés; cette réclamation, envoyée au Ministère par S.M. Louis XVIII, a été enregistrée sous le $n^{\circ} 13682$.

Je viens supplier Votre Excellence de vouloir bien me faire adresser, le plus tôt possible, une réponse officielle et définitive

J'ai l'honneur d'être | Monseigneur | De votre Excellence |Ce jour 13-1815 | Le tres-humble et tres-respectueux serviteur |Le comte de Gazola | rue Jacob Hôtel d'Hambourg faub. S. Germ. [faubourg Saint-Germain]

\section{DOCUMENT 63-31 JANVIER 1815}

Brouillon d'une lettre de l'abbé de Montesquiou, ministre de l'Intérieur, à Gazola (AN, F/17/1537, sous-dossier Gazola).

Le $M^{\text {tre }}$ [ministre]

31 jvier [janvier] 1815

À Mr le Cete de Gazola

$M^{r}$ le Cte, j'ai lu les deux lettres que vous m'avez fait l'hr [honneur] de m'écrire au sujet des collections d'objets d'bre nelle que vous possédiez en Italie et qui dans le temps furent transportées en France pour être dépôsées au Jardin du Roi à Paris.

Vous réclamez relativement au taux des indtés fixées rentes que vous avez reçus à titre d'indté [indemnité] pour les collections.

Mais, $M^{r}$ le Cte, cette affaire a été jugée il y a plus de 10 ans. C'est en 1803 qu'elle a été consommée. En 1808 elle a été confirmée encore et depuis cette époque vous avez joui des faveurs rentes sans éprouver d'obstacles

31 François-Xavier-Marc-Antoine, duc de MontesquiouFezensac (1756-1832). 
comme aussi sans élever de plaintes. Il existe même au dossier des lettres par lesquelles vous vous félicitez des ders [derniers] arrangements pris.

Je pense qu'il n'y a plus lieu à revenir sur cette affaire et quit fut je ne puis que la regarder comme entièrement terminée.

Quant aux droits particuliers que vous annoncez avoir aux bienfaits du gouvernement je vous estime heureux d'en avoir de pareils à faire valoir, mais c'est une chose distincte, saire te trater séparement et pour lequet vous pour laquelle il serait nécessaire de faire une demande spéciale et qui devrait être traitée séparément.

Je suis...

\section{DOCUMENT 64 - 18 FÉVRIER 1815}

Brouillon d'une lettre de l'abbé de Montesquiou, ministre de l'Intérieur, au baron Joseph-Dominique Louis, ministre des Finances (AN, F/17/1537, sous-dossier Gazola).

2224-3 fer [février] | Envoi d'une lettre de Mr le Cte de Gazola

Le Mtre [ministre] à Mr le Mtre des finances 18 fer [février] 1815

Mr le Bon [baron], j'ai l'honneur de vous faire le renvoi d'une lettre de $M^{r}$ le Cte [comte] de Gazola qui réclame au sujet de la suspension du payement de la pension de $2500 f$ dont il jouit sur le trésor public.

Cette pension créée en 1811[32] ne fut point ainsi que le dit $\mathrm{Mr}$ de Gazola une gratification pure \& simple, mais elle fut instituée comme le prix d'une collection considtérable de intéressante de poissons pétrifiés, collection qui fut apportée d'Italie et qui existe très utilement au muséum d'hre nelle [histoire naturelle] à Paris.

Je pense que le pay[ement] des 2500 assignés sur le trésor en faveur de $M^{r}$ de Gazola est entier [...] le cas d'être continué.

Je ne puis qu'inviter votre Ex ce [Excellence] à prendre à ce sujet des mesures favorables.

\section{DOCUMENT 65 - 7 MARS 1815}

Réponse du baron Joseph-Dominique Louis, ministre des Finances, à l'abbé de Montesquiou, ministre de l'Intérieur, donnant son accord pour maintenir la pension de Gazola (AN, F/17/1537, sous-dossier Gazola).

Ministère des Finances | Département de la dette inscrite | Bureau Central

Avis des ordres donnés pour que la pension de 2500 f. accordée à M. le Cte de Gazola lui soit continuée.

Paris, ce 7 mars 1815

32 C'est une erreur, cette pension fut créée en l'an 11 (1803) et non en 1811 .
Monsieur l'Abbé, j'ai reçu avec la lettre que votre Excellence m'a fait l'honneur de m'écrire le 18 du mois der, la pétition par laquelle $M$. le comte de Gazola demande à être maintenu, quoiqu'étranger, dans la jouissance de la pension de 2500.f. qui lui a été accordée par décret du 9 avril 1811 [33].

Votre excellence me confirmant que cette pension est le prix de la vente faite au gouvernement d'une collection intéressante de poissons pétrifiés dont il est en possession, j’ai donné les ordres nécessaires pour que la pension de M. le comte de Gazola lui soit continuée.

Je vous prie, Monsieur l'abbé, d'agréer l'assurance de ma haute considération.

Le ministre et secrétaire d'état des finances.

Le baron Louis

\section{DOCUMENT 66 - SANS DATE [VERS AVRIL-MAI 1815]}

Lettre de Gazola à Louis XVIII (AN, O/3/841, sous-dossier 3).

Sire,

Votre Majesté a daigné miaccueillir avec bonté, voudra-t-elle bien me permettre de demander grace. Le bonheur que jai éprouvé de la recevoir est tellement senti de moi et de ma famille, que j'ai décidée [sic] que l'habitation que j'ai a Verone où V. M. a résidé, serait non aliénable dans ma famille, voulant perpetuer un souvenir trop cher à mon coeur. Je suplie Votre Majesté de vouloir bien accorder au chef de la famille qui possedrà [sic] la maison susditte, le titre de commandant de la légion d'honneur. Cette faveur de Votre Majesté metra [sic] le comble à mon bonheur et à celui de ma famille.

DeVotre Majesté| Le tres-humbe, tres-obeissant et tres-devouéserviteur Le Comte de Gazola

\section{DOCUMENT 67 - 12 MAI 1815}

Rapport de Pierre Louis Jean Casimir de Blacas d'Aulps, ministre de la Maison du Roi, à Louis XVIII (AN, O/3/841, sous-dossier 3).

Gand, le 12 may 1815

Sire.

\section{Rapport}

J'ai l'honneur de proposer au Roi d'accorder le grade et la décoration de commandeur de la légion d'honneur à Mr le Comte de Gazola qui a donné à Votre Majesté des preuves de son zèle et de son attachement pour le Roi pendant son séjour de Votre Majesté à Veronne.

Signé Blacas d'Aulps

Ici est écrit de la main du Roi. Bon

Signé : Louis

Pour-copie conforme.

Le secrétaire général du ministère de la Maison du Roi.

33 Il s'agit du décret du 12 germinal an 11 (2 avril 1803). 
Catalogue des fossiles du cabinet Gazola remis à Louis Augustin Guillaume Bosc le 31 août 1803 par Giuseppe Rotari, commissaire de la municipalité de Vérone (Archives nationales F/17/1537). Les descriptions succinctes des spécimens et leurs croquis sont de la main de Bosc.

Catalogue des poissons pétrifiés remis au commissaire du gouvernement français Bosc par le commissaire de la municipalité de Veronne [sic] italienne

$1^{\circ}$ une raye de trois pieds de long sur un peu plus d'un de large soudée sur une pierre. Il y a les deux empreintes opposées.

$2^{\circ}$. Un brochet? de trois pieds de long sur trois pouces de large soudé sur une pierre. Il y a les deux empreintes opposées

$3^{\circ}$. Un brochet de deux pieds et demi de long sur trois pouces de large soudé sur une pierre. Il y a les deux empreintes opposées.

$4^{\circ}$. Un centropome? de deux pieds de long sur cinq pouces de large. Il y a les deux empreintes opposées.

$5^{\circ}$. Un genre nouveau de deux pieds de long sur un pouce de large. Il y a les deux empreintes mais elles ne sont pas opposées. L'autre n'a qu'un pied et demi.

6. Un chetodon d'un pied et demi de long sur huit pouces de large. Il n'y a qu'une empreinte.

$7^{\circ}$. Une raye de trois pieds trois pouces de long sur neuf pouces de large. Il y a les deux empreintes opposées

8. Un coryphene? d'un pied et demi de long sur cinq pouces de large. Il n'y a qu'une empreinte.

9. Un gade merlan d'un pied huit pouces de long sur quatre pouces de largeur à la tête. Il n'y a qu'une empreinte.

10. Un holacanthe? d'un pied et demi de long sur un demi pied de large. Il y a les deux empreintes opposées.

11 Un colyneme? de seize pouces de long sur quatre pouces de large. Il n'y a qu'une empreinte

12. Un holacanthe? d'un pied quatre pouces de long sur cinq pouces de large. Une seule empreinte.

13 Un chetodon d'un pied de long sur un demi de large. Deux empreintes opposées

14 Un holacanthe? d'un pied de long sur cinq de large. Deux empreintes opposées.

15. Un [...] d'un pied trois pouces de long sur deux pouces de large. Deux empreintes opposées.

16. Un [...] de deux pieds deux pouces de long sur trois pouces de large. Deux empreintes opposées.

17 Une murene d'un pied quatre pouces de long sur deux pouces de large. Il y a les deux empreintes opposées

18 Un [...] de deux pieds deux pouces de long, sur deux pouces de large. Il y a les deux empreintes opposées.

19. Le meme un peu plus petit. Il y a les deux empreintes opposées

20. Le même encore un peu plus petit. Ily a les deux empreintes opposées.

21 Encore le même plus petit. Ily a les deux empreintes opposées.
22. Un chetodon de six pouces de long sur un pied de large. Il $y$ a les deux empreintes opposées.

23. Un chetodon de neuf pouces de long sur six de large. Il y a les deux empreintes opposées.

24. Un bodian? d'un pied de long sur quatre pouces de large. Il y a les deux empreintes opposées mais elles ne sont pas opposées.

25. Un chetodon de huit pouces de long sur quatre de large. Il $y$ a les deux empreintes opposées.

26 Un zée de six pouces de long sur cinq de large. Il y a les deux empreintes opposées.

27. Un chetodon de huit pouces de long sur quatre de large. Il $y$ a les deux empreintes opposées.

28. Un chetodon de huit pouces de long sur quatre de large. Il $y$ a les deux empreintes opposées.

$29^{\circ}$. Un [...] d'un pied de long sur deux pouces de large. Il n'y a qu'une empreinte

$30^{\circ}$ Un [...] d'un pied de long sur un demi pouce de large. Il $y$ a les deux empreintes opposées.

$31^{\circ}$. Une murene d'un pied de long sur un pouce de large. Il y a les deux empreintes opposées.

$32^{\circ}$. Le meme genre nouveau du $n^{\circ} 5$ d'un pied de long sur un pouce de large. Il y a les deux empreintes opposées.

33. Une murene de dix pouces de long sur un de large. Il y a les deux empreintes opposées.

34. Une fistulaire d'un pied de long sur deux lignes de large. Il y a les deux empreintes opposées.

$35^{\circ}$. Une murene de huit pouces de long sur huit lignes de large. Il y a les deux empreintes opposées.

$36^{\circ}$. Une murene dont la tête est séparée du corps. Elle a un pied de long sur plus d'un pouce de large et sa double empreinte.

$37^{\circ}$. Une fistulaire de neuf pouces de long six lignes de large. Il $y$ a les deux empreintes opposées.

38. Un [...] de neuf pouces de long sur trois de large. Il y a les deux empreintes opposées.

39. Un [...] de neuf pouces de long sur deux de large. Il y a les deux empreintes opposées.

40. Un baliste? de neuf pouces de long sur cinq de large. Il n'y a qu'une empreinte

$41^{\circ}$. Un culpée? de neuf pouces de long sur un et demi de large. Il y a les deux empreintes

42․ Un bodian? de huit pouces de long sur deux de large. Il y a les deux empreintes opposées.

43. Le même de meme grandeur mais mieux conservé. Il y a les deux empreintes opposées.

$44^{\circ}$. Une murene de dix pouces de long sur un de large. Il y a les deux empreintes opposées.

$45^{\circ}$. Un bodian? de huit pouces de long sur trois de large. Ily a les deux empreintes opposées.

$46^{\circ}$. Un coryphene? de huit pouces de long sur deux de large. Il y a les deux empreintes opposées.

47․ Un clupé? de neuf pouces de long sur deux de large. Il y a les deux empreintes opposées 
48. Un [...] de huit pouces de long sur deux de large. Il y a les deux empreintes opposées.

49. Un [...] de sept pouces de long sur deux de large. Il y a une seule empreinte.

50. Un bodian? de sept pouces de long sur deux de large. Il n'y a qu'une seule empreinte

$51^{\circ}$. Une fistulaire de sept pouces de long sur deux lignes de large, la même que celle du $n^{\circ} 37$. Il y a les deux empreintes opposées.

52. Une scorpene? de sept pouces de long sur quatre de large. Il y a les deux empreintes opposées.

$53^{\circ}$. Un bodian? de cinq pouces de long sur trois de large. Il y a les deux empreintes opposées.

54. Un chetodon de trois pouces de long sur trois de large. Ily a les deux empreintes opposées

55. Deux spare et bodian? sur la même pierre. Il n'y a pas de double empreinte.

56 Un bodian? de cinq pouces de long sur deux de large. Il n'y a pas de double empreinte

57. Un chetodon de cinq pouces de long sur deux et demi de large. Il n'y a qu'une empreinte mais il y a deux pièces dont l'une est plus petite.

58. Un chetodon de sept pouces de long sur trois de large. Il y a les deux empreintes opposées.

59. Un bodian? de six pouces de long sur trois de large. Il y a les deux empreintes opposées

60. Un spare? de sept pouces de long sur un et demi de large. Il y a les deux empreintes.

61. Une murene de sept pouces de long sur six lignes de large. Il y a les deux empreintes

$62^{\circ}$. Un caranx de sept pouces de long sur deux de large. Il y a les deux empreintes opposées.

$63^{\circ}$. Une lophie de sept pouces de long sur un et demi de large. Il n'y a qu'une empreinte.

64. Deux petites spares? de deux pouces et demi de long sur moins d'un de large. Il y a les deux empreintes

65. Un chetodon? de six pouces de long sur trois de large. Ily a les deux empreintes opposées.

$66^{\circ}$. Un fistulaire de quatre pouces de long sur quatre lignes de large. Il y a les deux empreintes

$67^{\circ}$. Un bodian? de six pouces de long sur deux de large. Il y a les deux empreintes opposées

$68^{\circ}$. Une lophie de six pouces de long sur un de large. Il y a les deux empreintes opposées.

69. Un [...] de six pouces de long sur un peu plus d'un pouce de large. Il y a les deux empreintes opposées.

$70^{\circ}$. Un [...] de six pouces de long sur un peu plus d'un pouce de large. Il y a les deux empreintes opposées.

$71^{\circ}$. Un coryphene? de quatre pouces de long sur un et demi de large. Il n'y a qu'une empreinte

$72^{\circ}$. Un spare? de cinq pouces et demi de long sur un et demi de large. Il n'y a qu'une empreinte

$73^{\circ}$. Deux spares? sur la même pierre. Il n'y a qu'une empreinte.

74 quatre spare? sur la même pierre. Il n'y a qu'une empreinte.
75․ Une sciene? de quatre pouces de long sur un peu plus d'un de large. Il y a deux empreintes opposées.

$76^{\circ}$. Une scorpene? de trois pouces de long sur un de large. Il $y$ a les deux empreintes opposées.

$77^{\circ}$ Un holocentre? de cinq pouces de long sur trois de large. Il y a les deux empreintes opposées.

$78^{\circ}$. Un chetodon de deux pouces de long sur quatre de large. Il y a les deux empreintes opposées

$79^{\circ}$ Un holocentre? de cinq pouces de long sur deux de large. Il y a les deux empreintes opposées.

$80^{\circ}$. Un du même genre de six pouces de long sur deux de large. Il y a les deux empreintes opposées.

$81^{\circ}$ Trois sur la même pierre du même genre que le $n^{\circ} 74$. Ily a les deux empreintes

$82^{\circ}$. Un chetodon de cinq pouces de long sur quatre de large. Il y a les deux empreintes opposées.

$83^{\circ}$. Un [...] de six pouces de long sur un de large. Il n'y a qu'une empreinte.

84․ Le même de même grandeur mais d'une moins bonne conservation. Il y a deux empreintes.

$85^{\circ}$ Un chetodon de six pouces de long sur trois de long. Il y a les deux empreintes opposées

86. $6^{\circ}$ Un achanthure [sic] de six pouces de long sur deux et demi de large. Il n'y a qu'une seule empreinte.

87. Un labre? de cinq pouces de long sur deux de large. Il y a les deux empreintes opposées

88. Un fistulaire de cinq pouces de long sur trois lignes de large. Il y a les deux empreintes opposées.

$89^{\circ}$ Un spare? de quatre pouces de long sur un et demi de large. Il y a les deux empreintes opposées.

$90^{\circ}$. Un holocentre? de cinq pouces de long sur deux de large. Il y a les deux empreintes opposées.

$91^{\circ}$. Un [...] de cinq pouces de long sur deux et demi de large. Il y a les deux empreintes opposées.

$92^{\circ}$. Un holocentre? de quatre pouces de long sur deux de large. Il y a les deux empreintes opposées.

93․ Un holocentre? de quatre pouces et demi de long sur un et demi de large. Il y a les deux empreintes opposées.

$94^{\circ}$. Un [...] de cinq pouces de long sur un et demi de large. Il $y$ a les deux empreintes opposées.

95. Un holocentre? de cinq pouces de long sur près de deux de large. Il y a les deux empreintes opposées.

96. Un esoce de six pouces de long sur un de large. Il n'y a qu'une seule empreinte.

97. Un acanthure? de cinq pouces de long sur deux de large. Il n'y a qu'une seule empreinte.

98. . Trois petits [...] sur la même pierre. Il n'y a qu'une seule empreinte

99. Une sciene? de cinq pouces de long sur deux de large. Ily a les deux empreintes opposées.

100 Un esoce? de cinq pouces de long sur un de large. Il y a les deux empreintes opposées.

101. Un holocentre? de quatre pouces de long sur deux de large. Il y a les deux empreintes opposées. 
102 Une sciene? de quatre pouces de long sur un et demi de large. Il n'y a qu'une empreinte.

103. Un holocentre? de trois pouces de long sur un peu plus d'un de large. Il y a les deux empreintes opposées.

104. Un chetodon de deux pouces de long sur quatre de large. Il y a les deux empreintes opposées.

105. Un chetodon glaucus de trois pouces de long sur deux de large. Il y a les deux empreintes opposées.

106. Un holocentre de quatre pouces de long sur deux de large. Il y a les deux empreintes opposées.

107. Un holocentre? de quatre pouces de long sur deux de large. Il y a les deux empreintes opposées.

108. Un holocentre? de quatre pouces de long sur deux de large. Il y a les deux empreintes opposées.

109. Un spare? de quatre pouces de long sur un de large. Il y a les deux empreintes opposées.

110. Le même un peu plus grand et plus noir. Il y a les deux empreintes opposées.

110bis une blénie? de cinq pouces de long sur un de large. Il $y$ a les deux empreintes opposées.

111. Un esoce? de quatre pouces de long sur un demi de large. Il y a les deux empreintes opposées.

112. Le même un peu plus grand. Il n'y a qu'une empreinte.

113. Un [...] de quatre pouces de long sur un peu plus d'un demi de large. Il n'y a qu'une seule empreinte.

114. Le même que le $n^{\circ}$. 112. Il n'y a qu'une empreinte.

115. Un [...] de quatre pouces de long sur un de large. Il y a les deux empreintes opposées.

$116^{\circ}$. Un centropome? de quatre pouces de long sur un de large. Il y a les deux empreintes opposées.

117. Un spare? de quatre pouces de long sur un et demi de large. Il n'y a qu'une seule empreinte

118. Un [...] de quatre pouces de long sur un demi de large. Il $y$ a les deux empreintes opposées.

119. Un spare? de quatre pouces de long sur un de large. Il n'y a qu'une empreinte

120. Un spare? de quatre pouces de long sur un et demi de large. Il y a les deux empreintes opposées.

121. Un chetodon glaucus? de quatre pouces de long sur deux de large. Il y a les deux empreintes opposées. Voyez n ${ }^{\circ} 105$.

122. Un du genre nouveau du $n^{\circ} 5$ n'ayant que six pouces de long sur trois lignes de large. Il y a les deux empreintes.

123. Un chetodon cornutus? de quatre pouces de long sur deux de large. Il y a les deux empreintes opposées.

124 Un pegase? de trois pouces de long sur trois lignes de large. Il y a les deux empreintes opposées.

125. Un chetodon de deux pouces de long sur trois de large. Il $y$ a les deux empreintes opposées.

126. Un scorpene? de quatre pouces de long sur deux de large. Il y a une seule empreinte.

127. Un labre? de quatre pouces de long sur un et demi de large. Il n'y a qu'une seule empreinte.

128. Un [...] de deux pouces de long sur deux lignes de large. Il $y$ a les deux empreintes opposées.
129. Le meme chetodon du $n^{\circ} 125$ mais plus petit et mieux conservé. Il y a les deux empreintes.

130. Un ostracion de deux pouces de long sur deux de large. Il $y$ a les deux empreintes opposées.

131. Un chetodon de trois pouces de long sur quatre de large. Il y a les deux empreintes opposées.

132. Quatre petits spares ou bodians? sur la même pierre. Il y a deux empreintes opposées.

133. Un chetodon canesceus? de quatre pouces de long sur quatre de large. Il y a deux empreintes opposées.

134. Le même un peu plus petit.

135. Une scorpene? de trois pouces de long sur deux de large. Il n'y a qu'une empreinte.

136. Un spare? de trois pouces de long sur un de large. Il y a les deux empreintes opposées.

137. Un esoce de quatre pouces de long sur six lignes de large. Il y a les deux empreintes opposées.

138. Un [...] de trois pouces de long sur un demi de large. Il y a les deux empreintes opposées.

139. Un bodian? de trois pouces et demi de long sur un et demi de large. Il n'y a qu'une empreinte.

140. Un [...] de deux pouces et demi de long sur un de large. Il n'y a qu'une empreinte.

141. Le pegase dragon de trois pouces de long sur six lignes de large. Il n'y a qu'une empreinte.

142. Un [...] de deux pouces et demi de long sur un peu plus d'un demi de large. Il y a les deux empreintes opposées.

143. Un chetodon d'un pouce et demi de long sur un de large. Il y a les deux empreintes opposées.

144 Un [...] d'un pouce de long sur six lignes de large. Il n'y a qu'une empreinte.

145. Un holocentre? de deux pouces de long sur un de large. Il n'y a qu'une empreinte.

146. Un chetodon d'un pouce de long sur autant de large. Il y a les deux empreintes.

147. Un chetodon d'un pouce de long sur autant de large. Il y a les deux empreintes opposées.

148. Le même mais sans double empreinte.

149. Une murene de six pouces de long sur trois lignes de large. Il y a les deux empreintes

150. Une plante sans double empreinte.

151. Une plante avec une double empreinte.

152. Une plante sans double empreinte.

153. Une plante avec une double empreinte.

154. Une feuille avec une double empreinte.

155. Une feuille avec une double empreinte.

156. Des feuilles en faisceau. Double empreinte.

157. Une feuille digitée. Il y a une double empreinte.

158. Une plante. Il n'y a pas de double empreinte.

159. Une plante. double empreinte.

160. Une plante sans double empreinte.

161. Un fruit. double empreinte.

162. Une plante. double empreinte.

163. Une plante. double empreinte. 
164. Une plante. double empreinte.

165. Un grand crustacé voisin de la langouste

166. Un autre du genre occipode?

Petrifications qui n'appartiennent pas au Mont Bolca

167 Une agregation d'os de quadrupèdes parmi lesquels se distingue une machoire

168 Une autre semblable mais plus petite

169 Un agrégat de plusieurs espèces de coquilles

170 un agregat de balanes

171 un moule intérieur de nautile.

172 un tubipore a grands trous

173 un tubipore a petits trous

174 Quatre crabes se rapprochant des calappes ou des dromies

175 Quatre exemplaires du strombe de val de Ronca

176 Un crabe voisin des grapses

177 Deux cérites de Ronca

178 Deux turritelles

179 Une cardite

180 Deux peignes

181 trois ammonites pyriteuses

182 une térebratule
183 une bucardite

184 Deux grosses dentales

185 Deux mitres

186 une autre

187 Une pyrule

188 Quatre vermiculaires

189 Deux madrepores fongite?

190 Deux crustacés dont un du genre galathée

191 un poisson du Mont Liban

Moi soussigné membre de l'administration municipale de la ville de Verone italienne et commissaire nommé par elle pour remettre au $C^{n}$ Bosc commissaire de gouvernement francois une collection de poissons fossiles et autres objets petrifiés qu'elle a offert au Premier Consul de la République Francoise par l'organe de $C^{n}$ Gazola son député a Paris et qui a été accepté par arrêté de gouvernement Francois du 12 germinal an XI, certifie que le catalogue cÿ dessus est celui des articles que comprend cette collection les quelles ont été bien et duement emballé en ma présence

Faite a Veronne le 31 aout 1803 an 2 de la Republique italienne Giuseppe Rotari

$\mathrm{Vu}$; le Ministre de l'intérieur 
fice $170: 3$.

Catabogue on poillon pelvifién semis ace

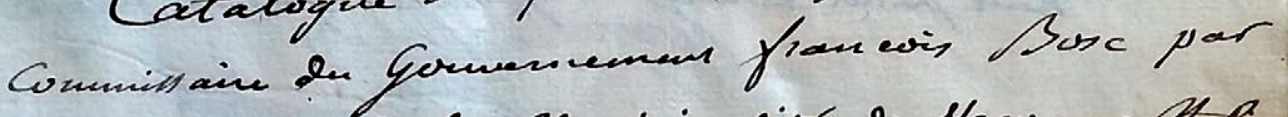

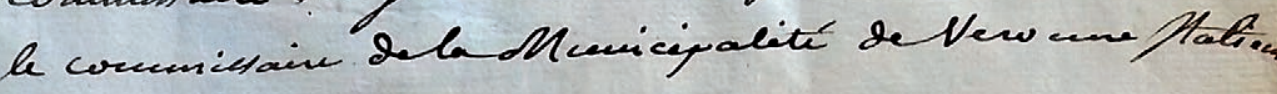

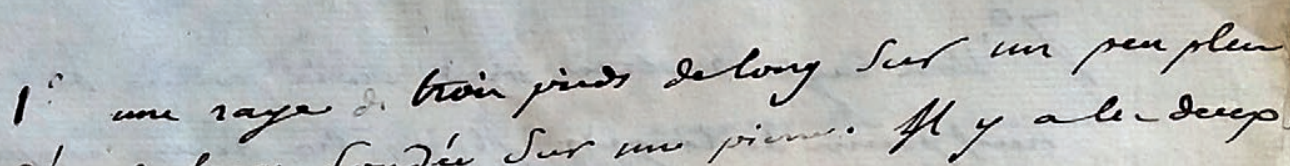

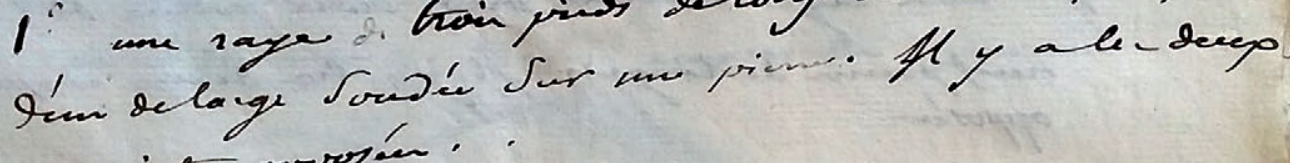
cuprainter opgrosien.

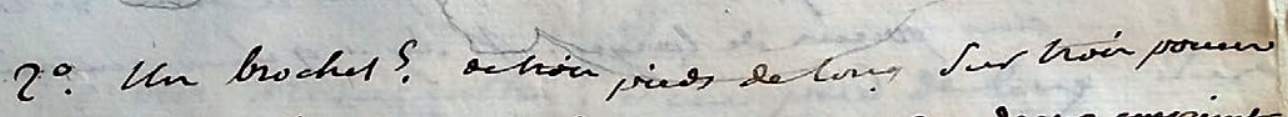

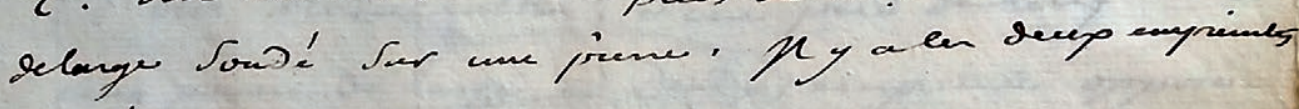
oppoien

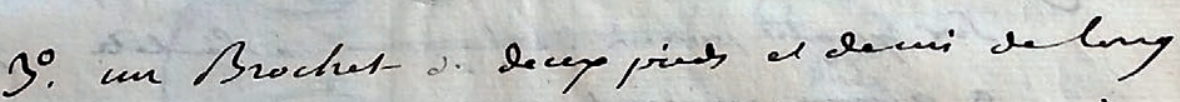

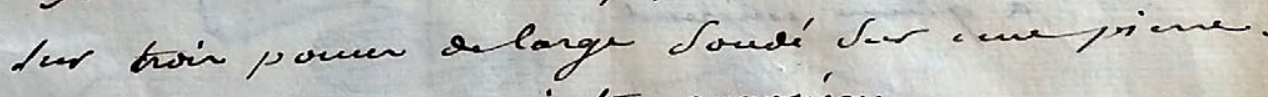

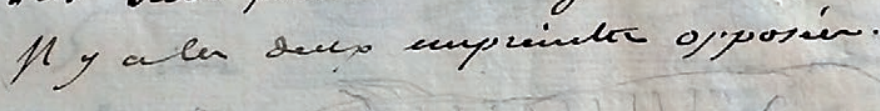

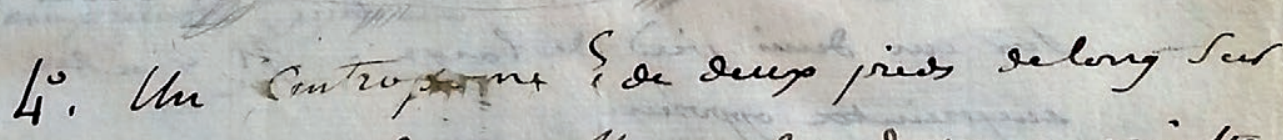

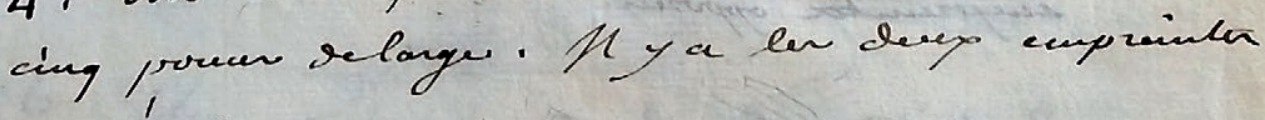
ojporeen:

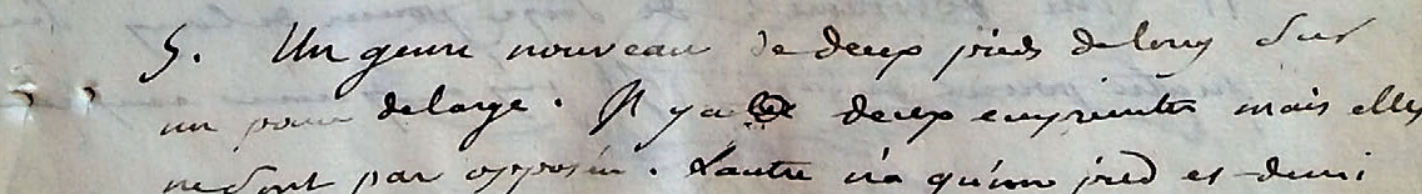

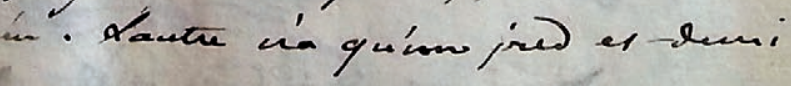




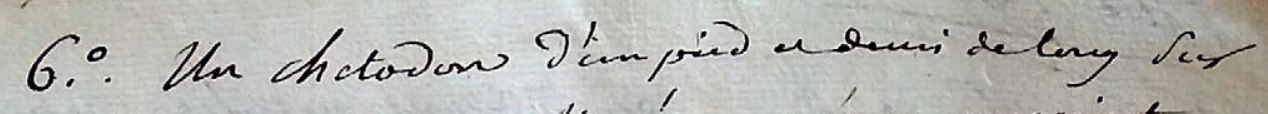

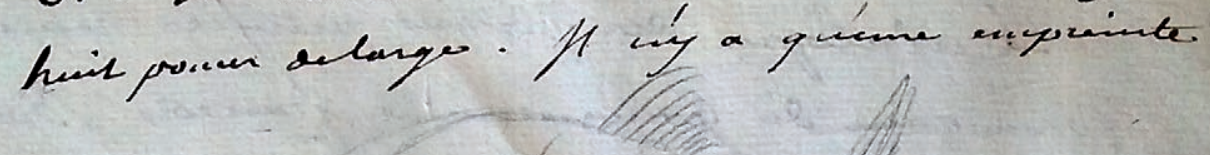

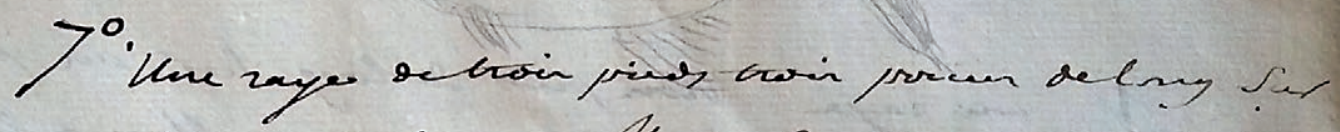

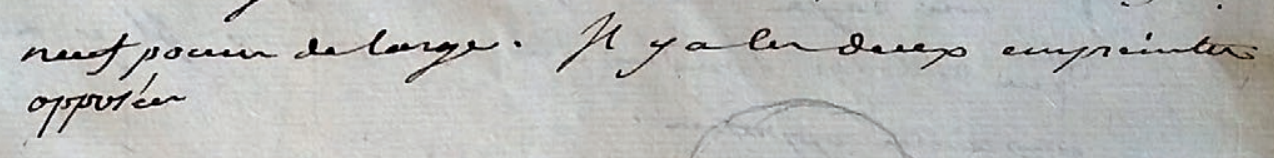

8. Un conptienes J'm

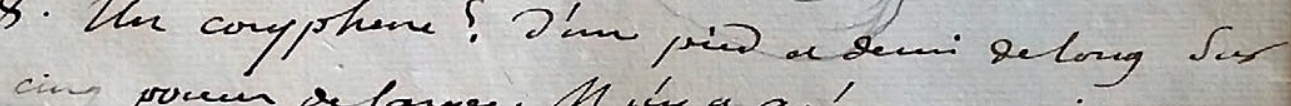

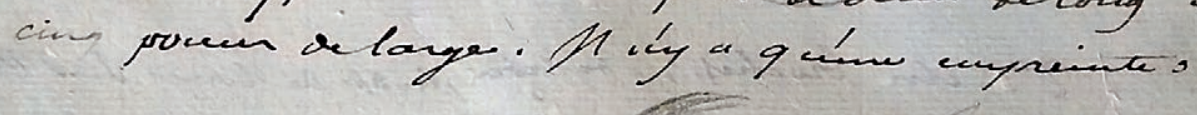
(O)

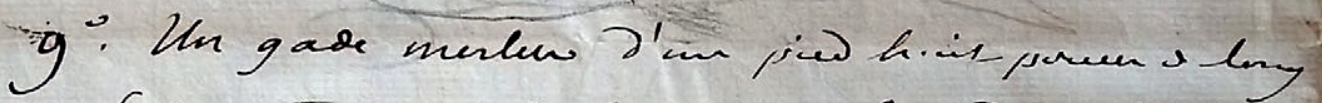

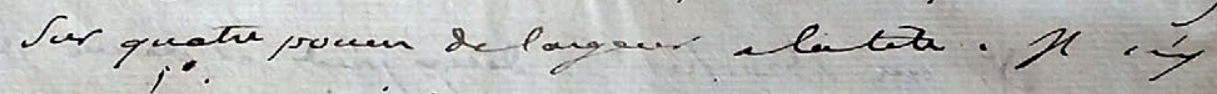

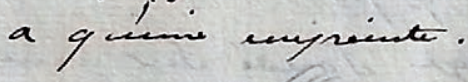

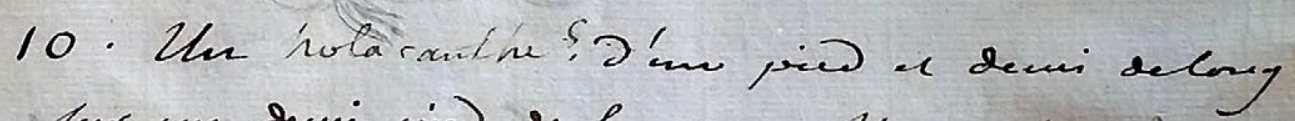

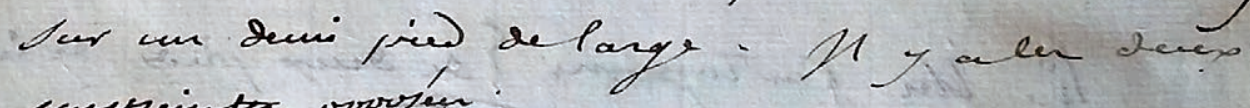
supreainta pprosen

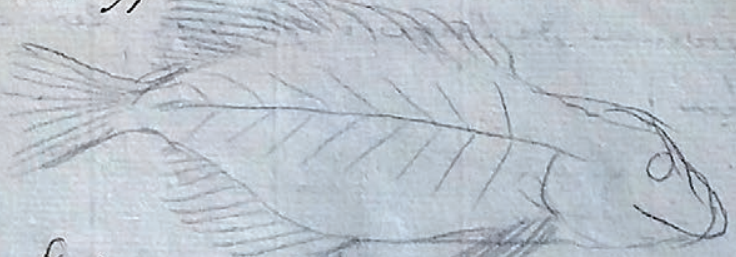

II Un bolynemes or fize poumen de lang fer

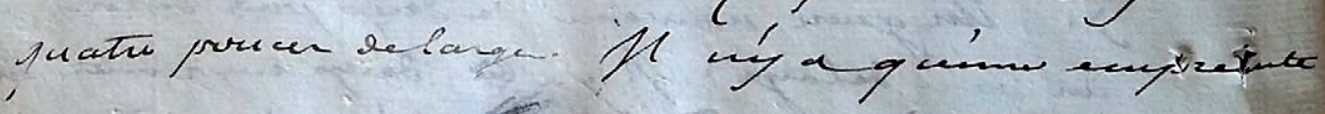

ANNEXE 2. - Suite; catalogue de Louis Augustin Guillaume Bosc, spécimens 6 à 11 . 


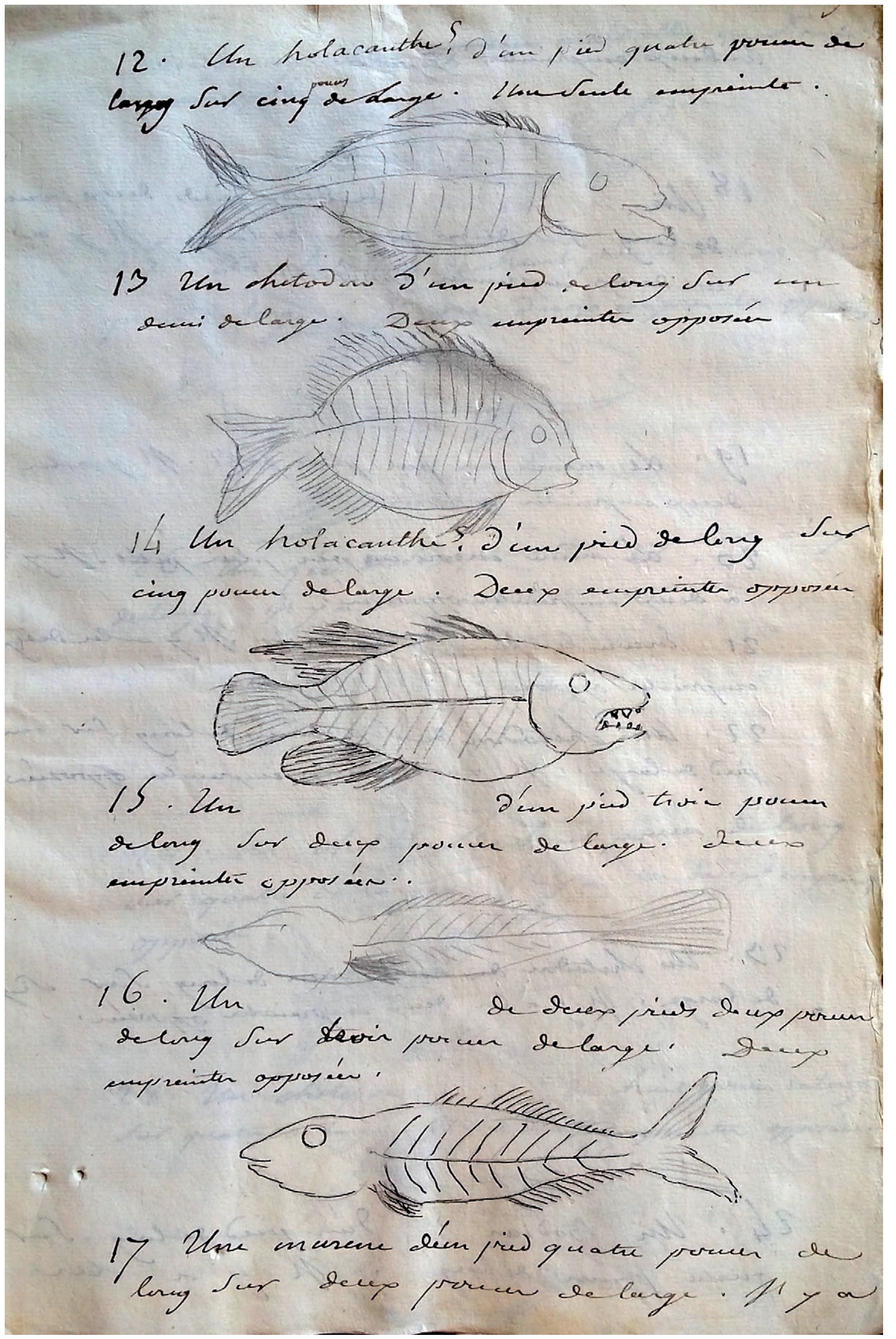

ANNEXE 2. - Suite; catalogue de Louis Augustin Guillaume Bosc, spécimens 12 à 17. 
- Brignon A.

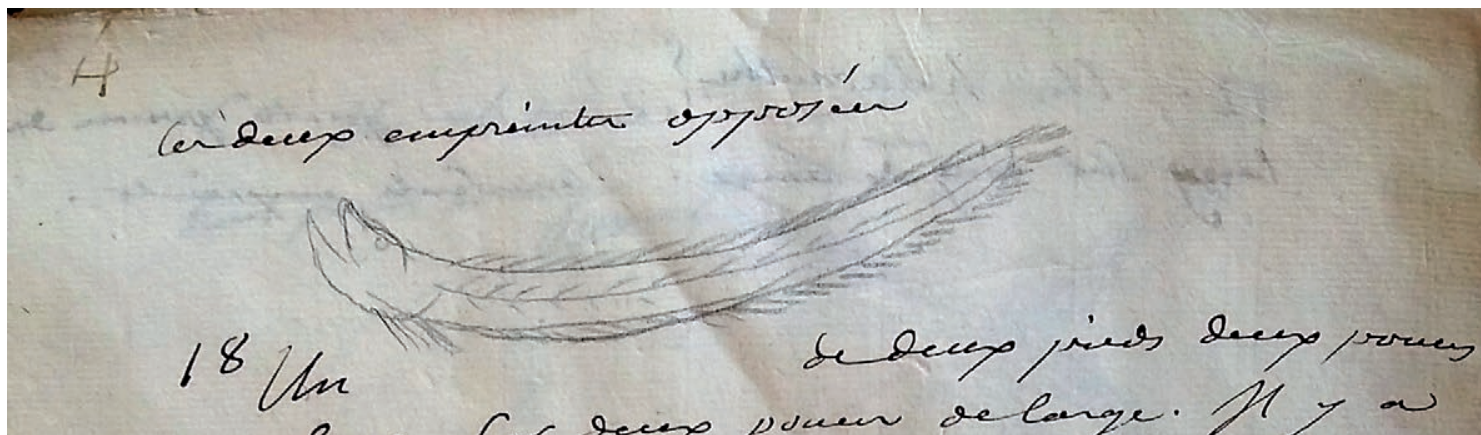

deling, far deas pouen de lange. My a ler deago anginder opporén.

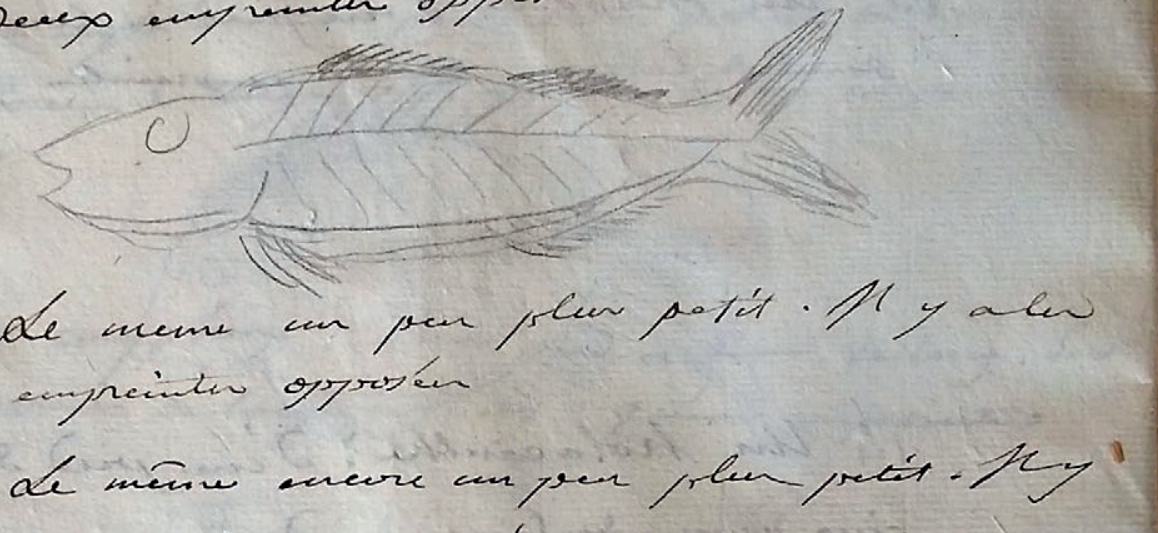

19. de nume
decep supreintu
20. de manne

a decep cuprainter oppos ér.

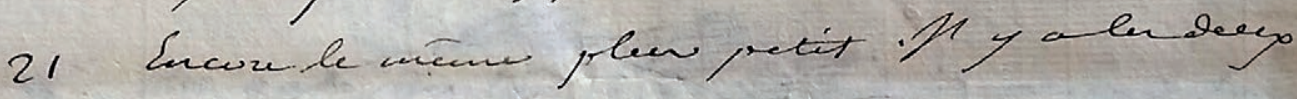
rayrainder opprosén.

22. Un chetobut or sip precen dacky for

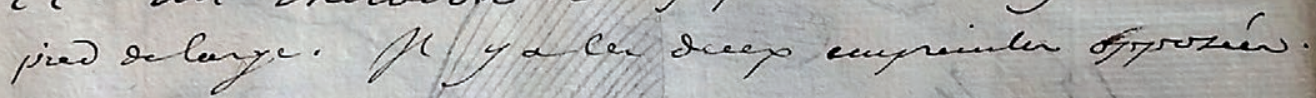

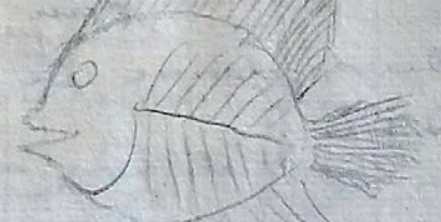

23. Un chetorom de rent proven decong for is

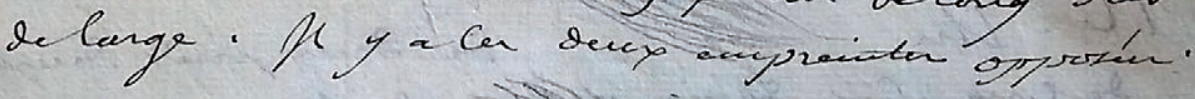

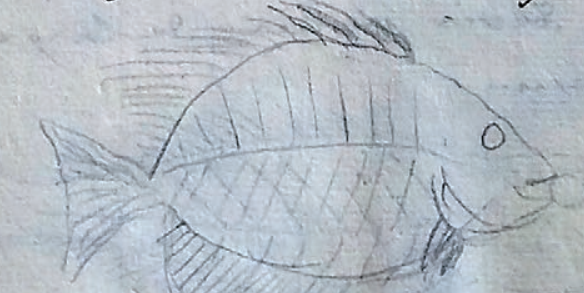

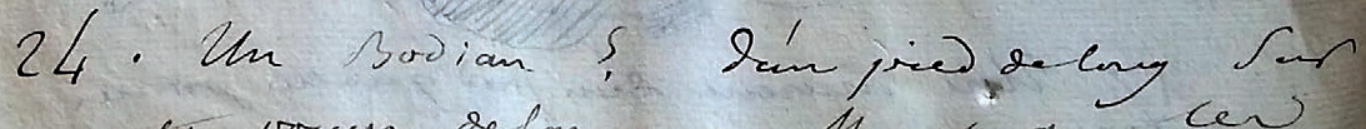
quale powen delarge. $\mu$ is a cer

ANNEXE 2. - Suite; catalogue de Louis Augustin Guillaume Bosc, spécimens 18 à 24.

96

GEODIVERSITAS • $2019 \cdot 41$ (2) 


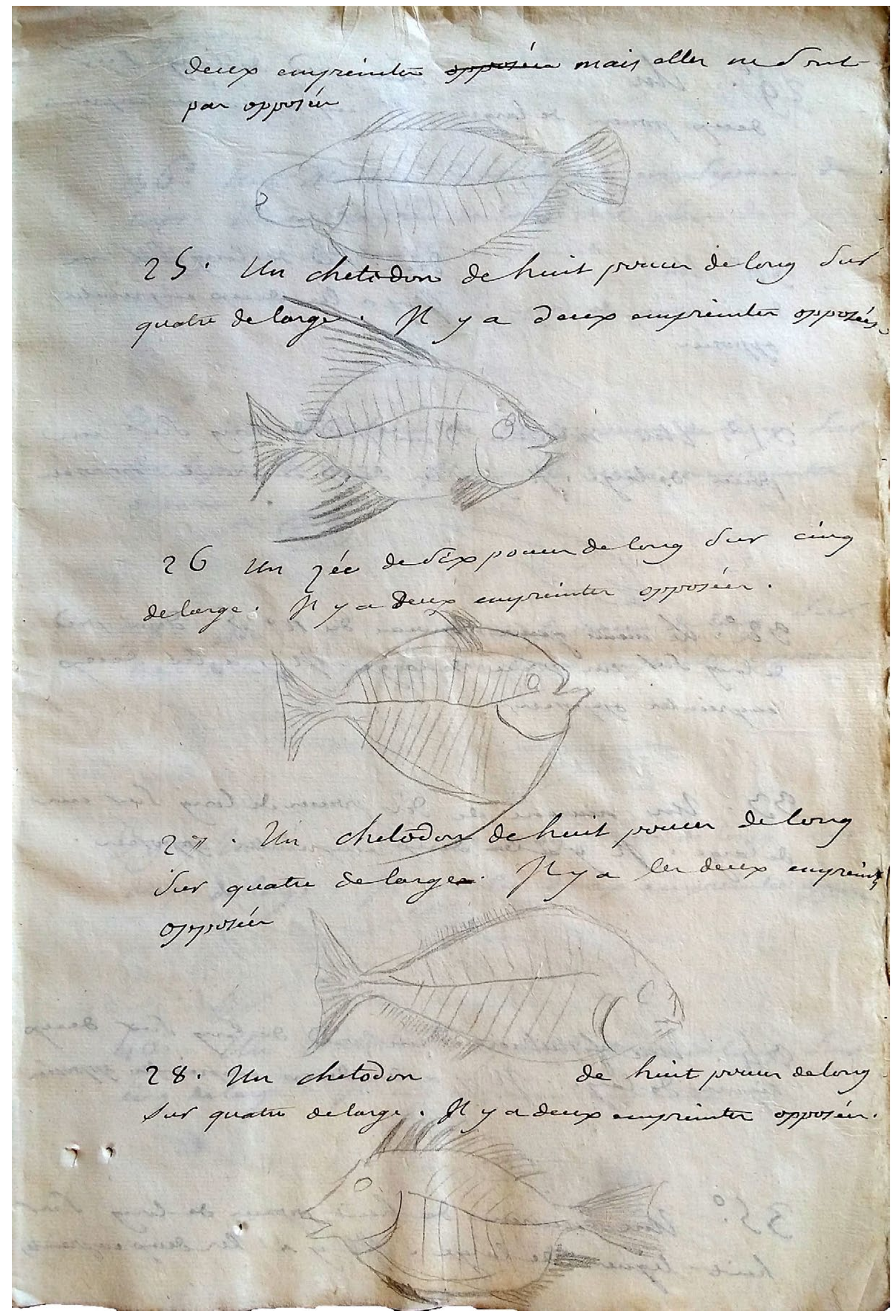

ANNEXE 2. - Suite; catalogue de Louis Augustin Guillaume Bosc, spécimens 25 à 28. 
- Brignon A.

$2 g^{\circ}$. Un dempres deling ler

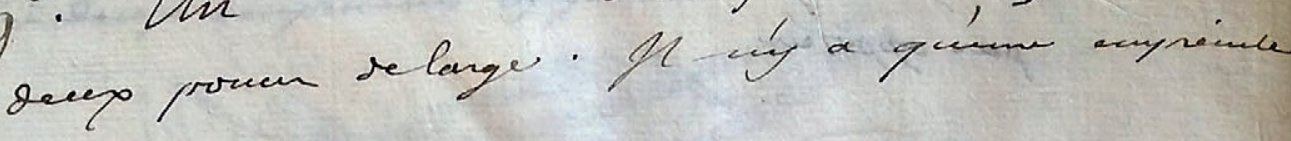

$30^{\circ} \mathrm{Hm}$

dín jios de lang for m

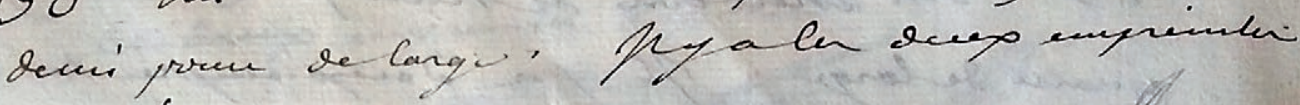
oppozín.

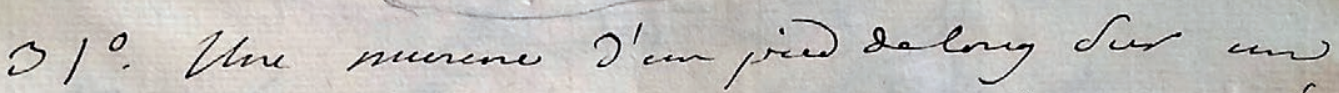

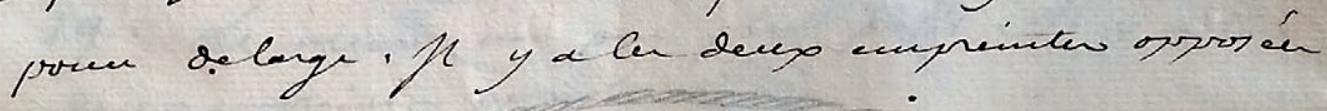

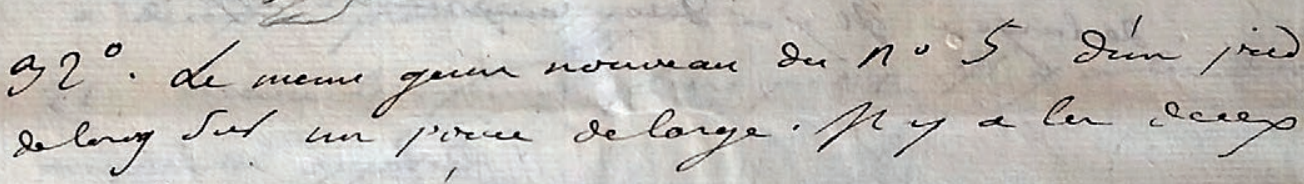

cayseculer opyrocén.

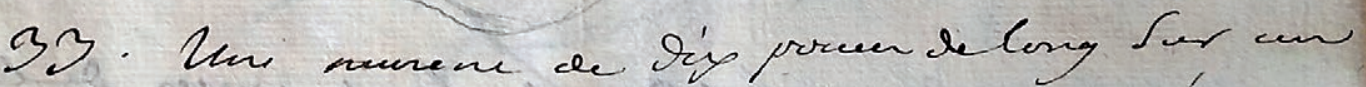

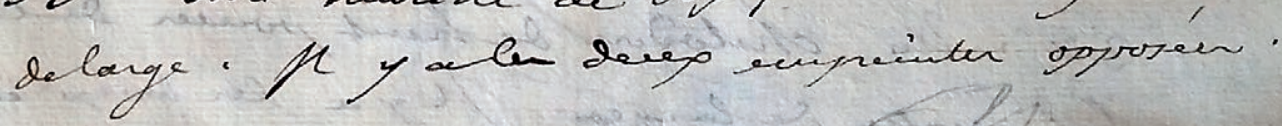

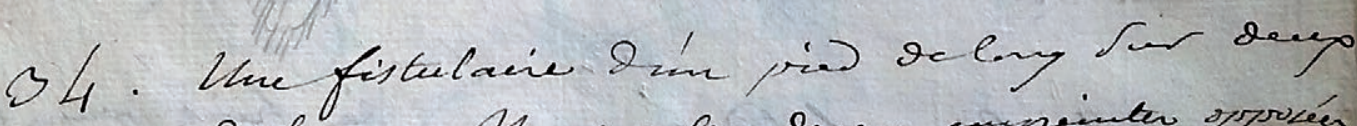

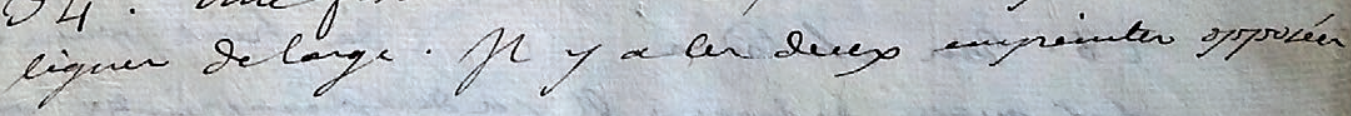

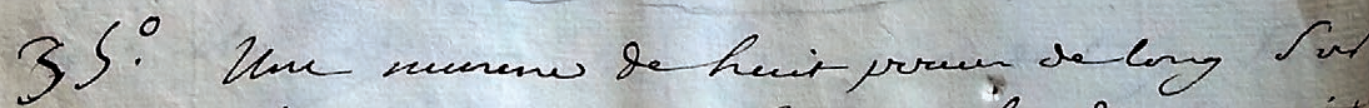

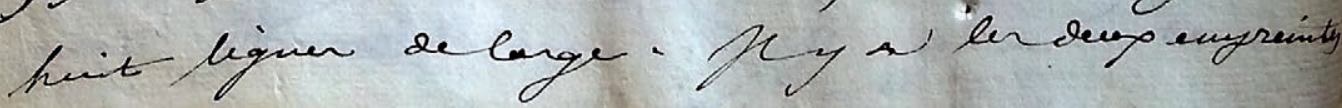

ANNEXE 2. - Suite; catalogue de Louis Augustin Guillaume Bosc, spécimens 29 à 35.

98

GEODIVERSITAS • 2019 • $41(2)$ 


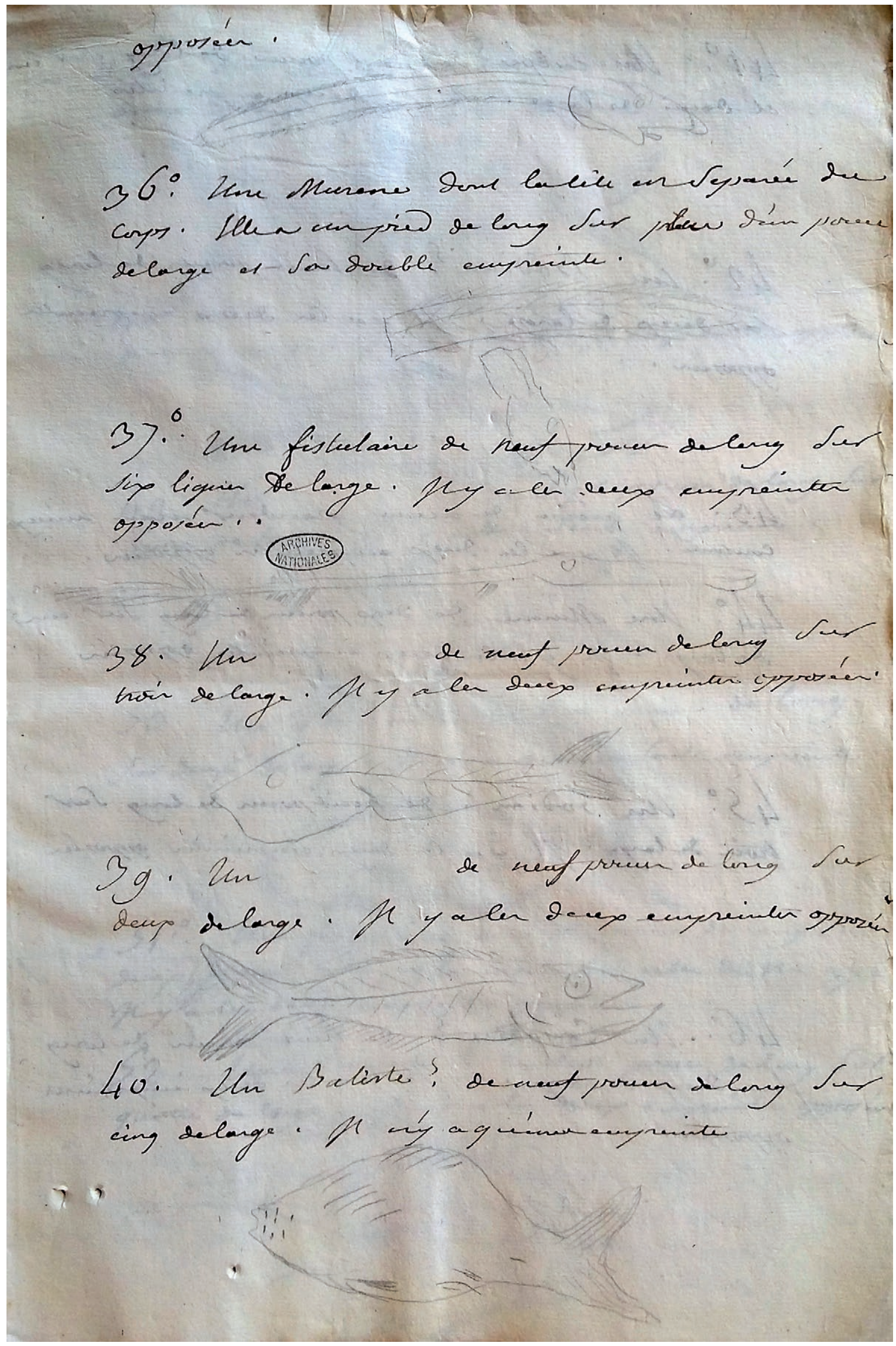

ANNEXE 2. - Suite; catalogue de Louis Augustin Guillaume Bosc, spécimens 36 à 40. 
- Brignon A.

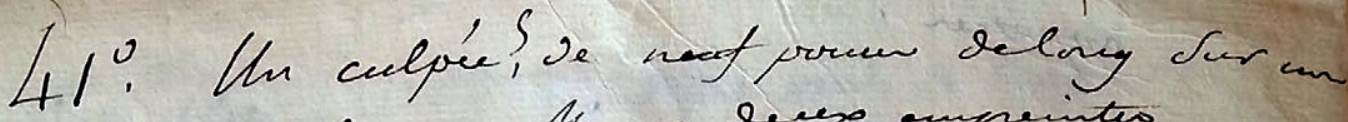

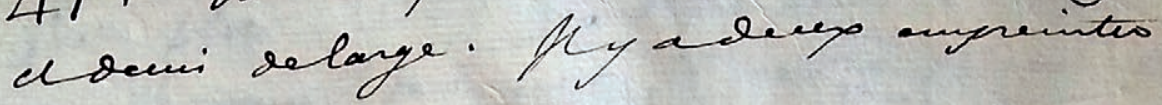

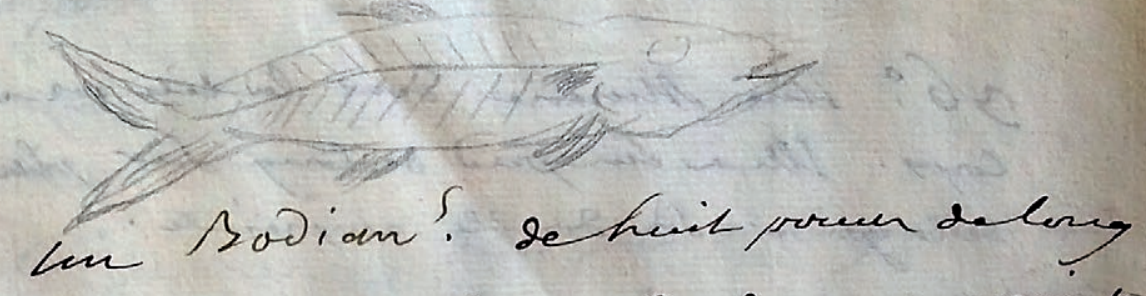

42. Un Bobian! de hait presen dalery

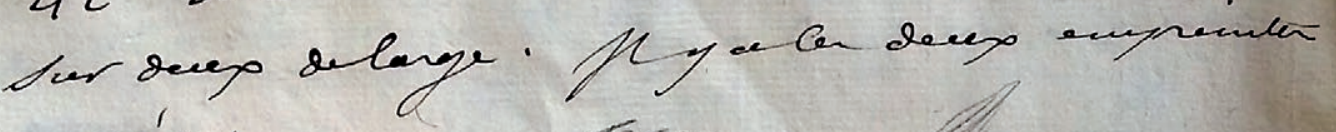
opgroún.

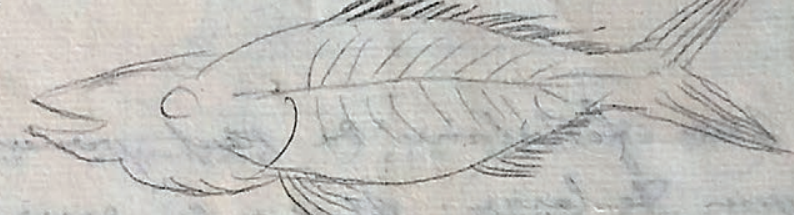

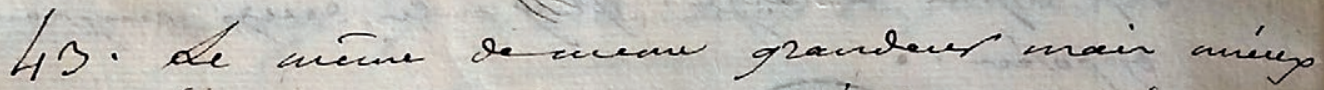

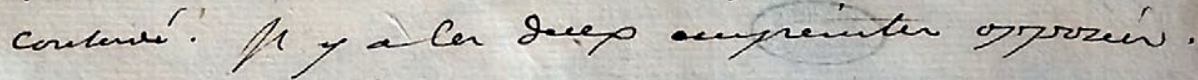

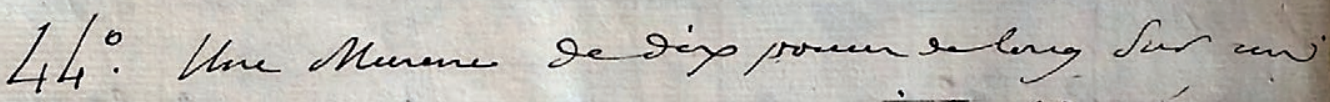

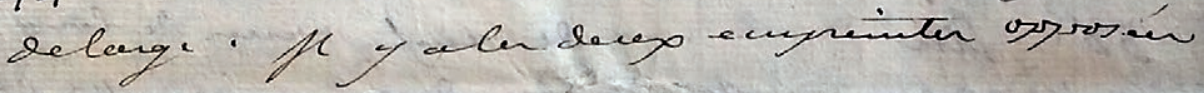

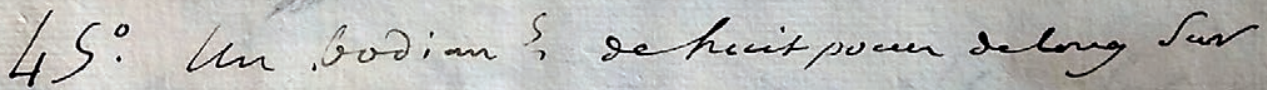

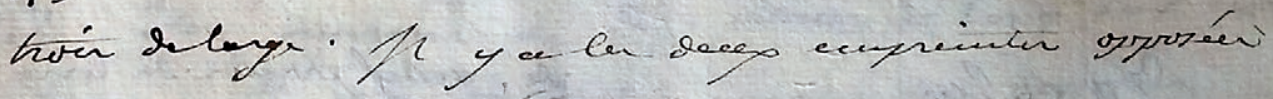

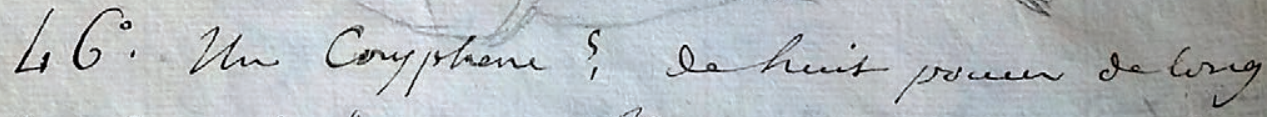

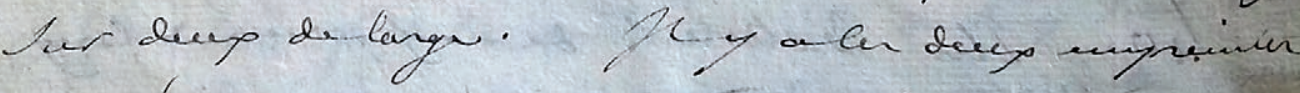
ofprosen.

ANNEXE 2. - Suite; catalogue de Louis Augustin Guillaume Bosc, spécimens 41 à 46.

100

GEODIVERSITAS • $2019 \cdot 41$ (2) 


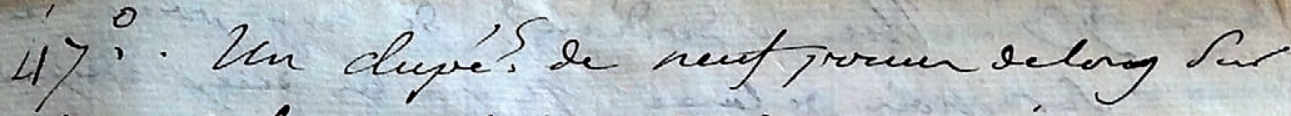
derep delayge: $\mathrm{fr}$ a lev deces enpreinter opporés $48.2 m$

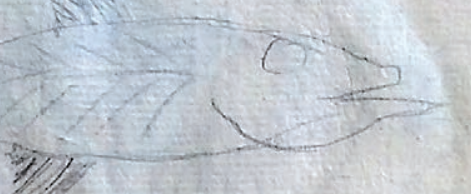

decep de cargu.

le huil prous

de long dar

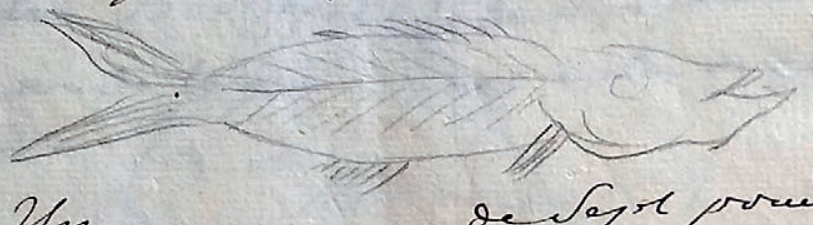

$49 \cdot 2 \mathrm{ln}$

de Segr prowen delang far

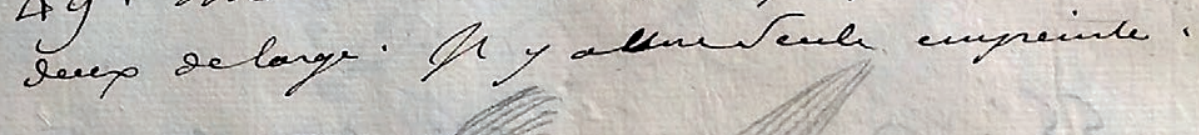

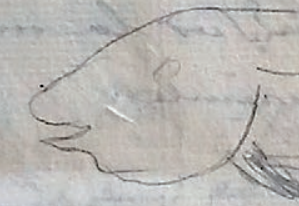

So. Un Bobian? de Sept, rovin deling

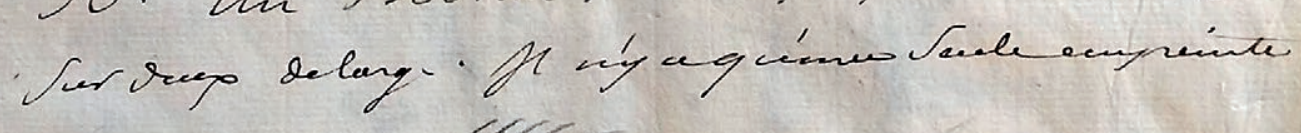

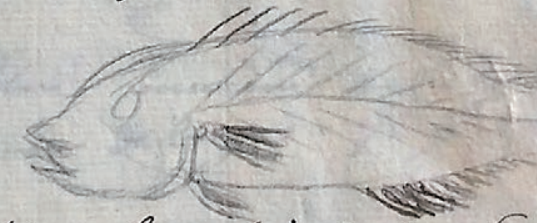

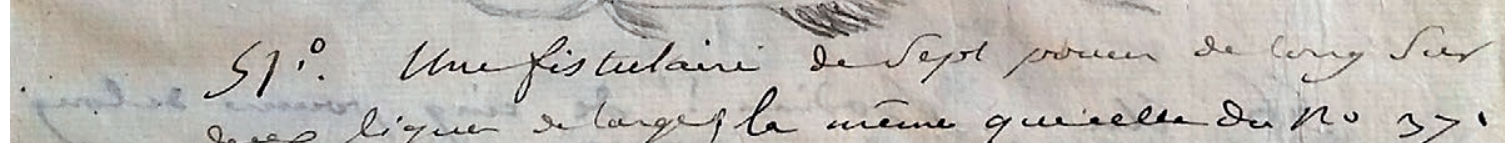

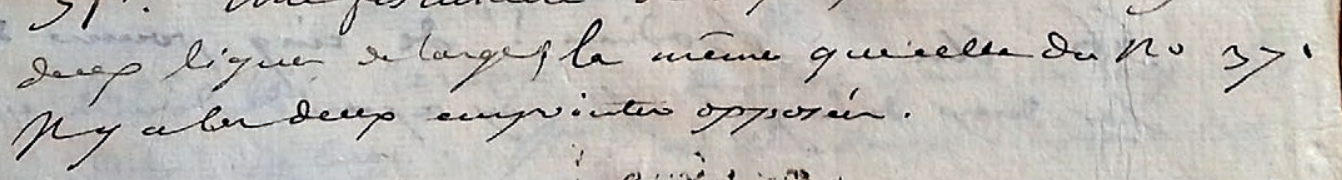

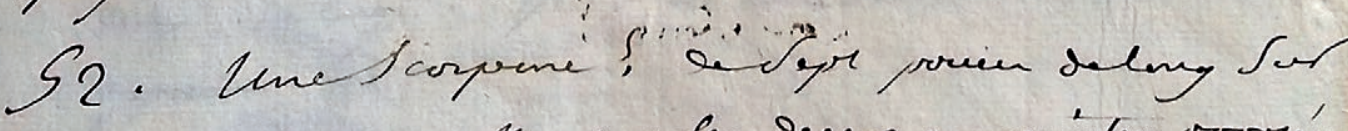

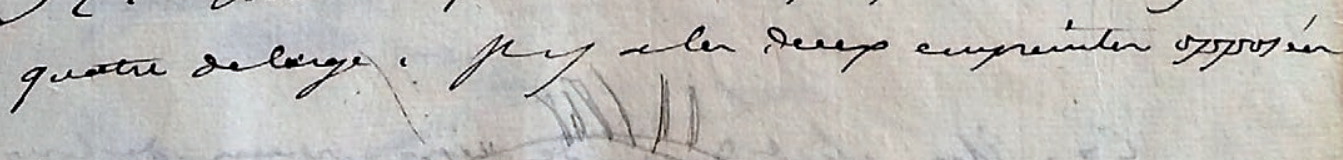

79

ANNEXE 2. - Suite; catalogue de Louis Augustin Guillaume Bosc, spécimens 47 à 52. 
S3: Un Botian? de cing prean delang

Sor noir relange. Jy a an ding enyrintas oproicen.

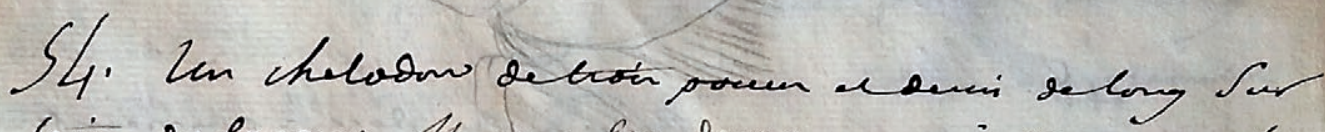

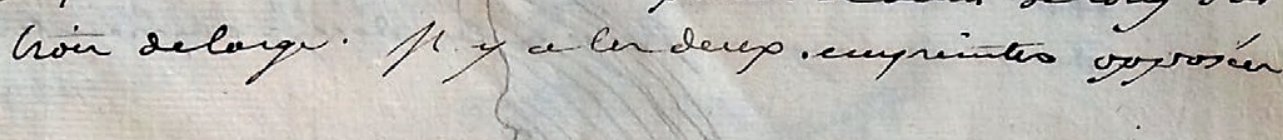

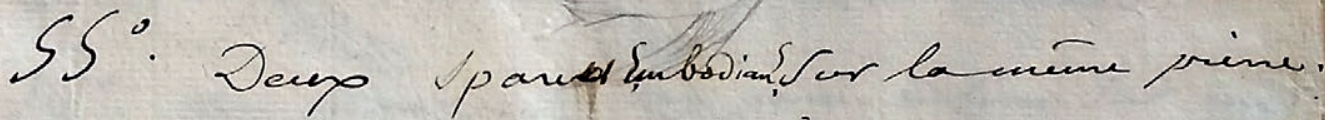

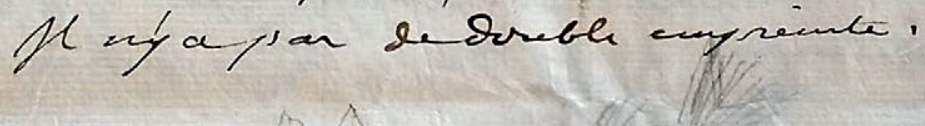

$56 \mathrm{th}$ Avolian! de cing premen selong

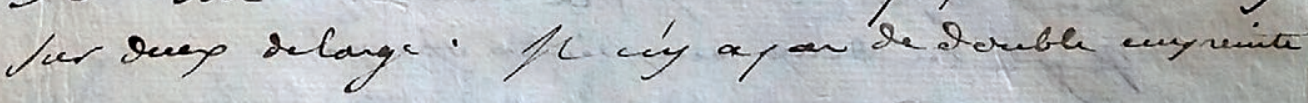

5). Un cheladow de cing prouen, decong for

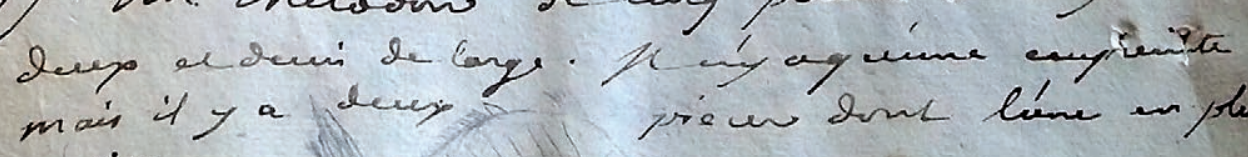
patite.

ANNEXE 2. - Suite; catalogue de Louis Augustin Guillaume Bosc, spécimens 53 à 57. 
Les conditions d'acquisition de la collection Gazola par le Muséum national d'Histoire naturelle

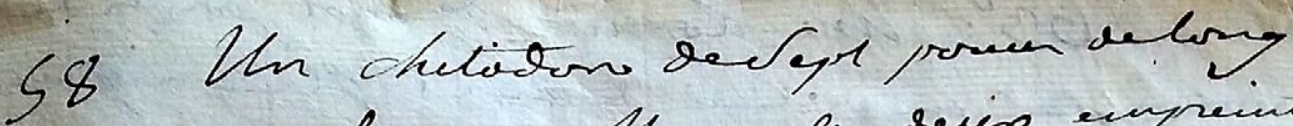

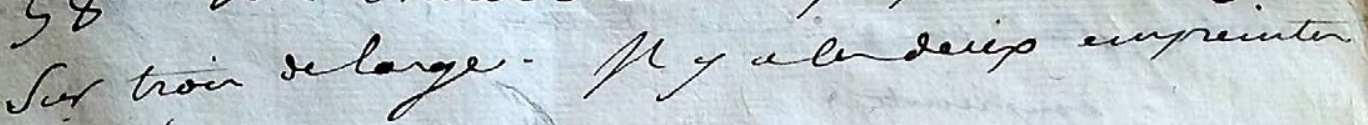
siforién.

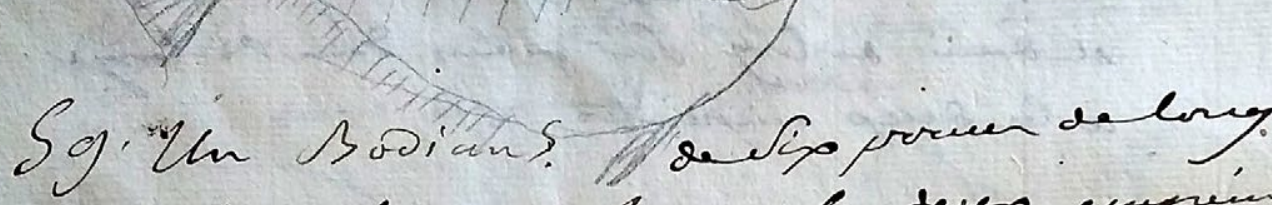

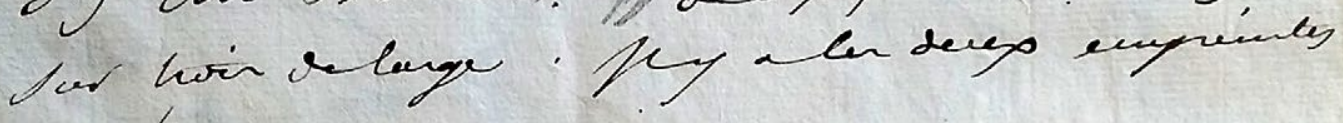
orporín

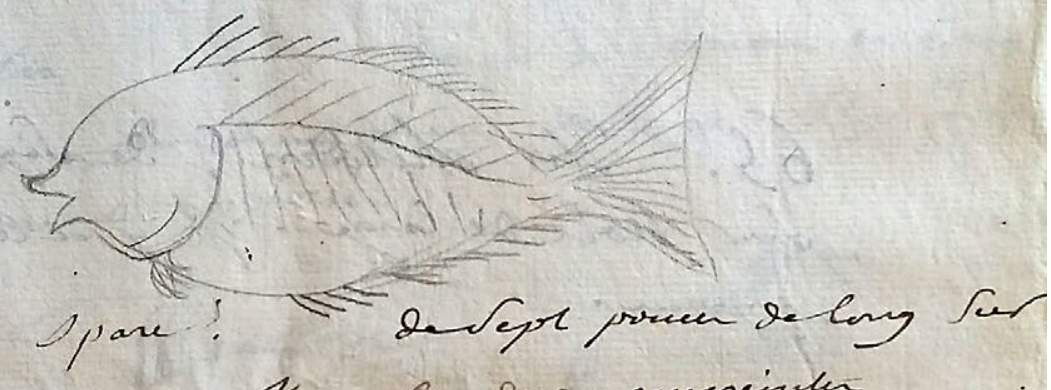

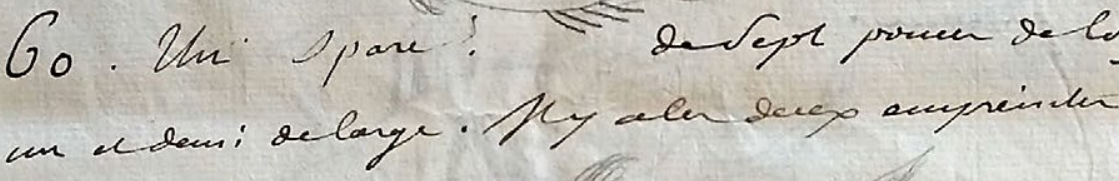

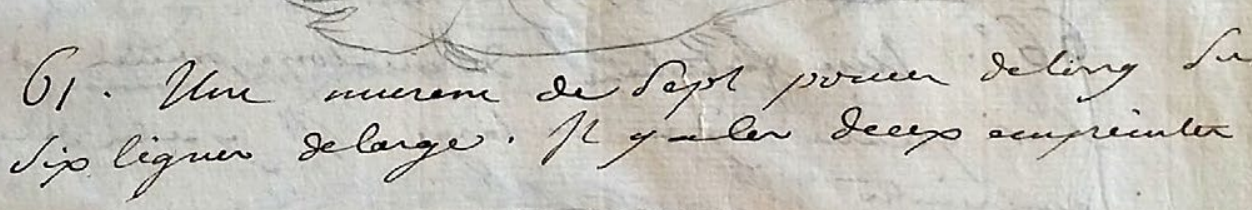

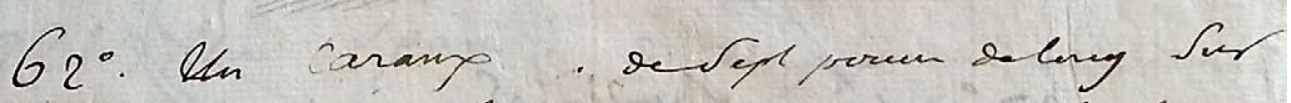

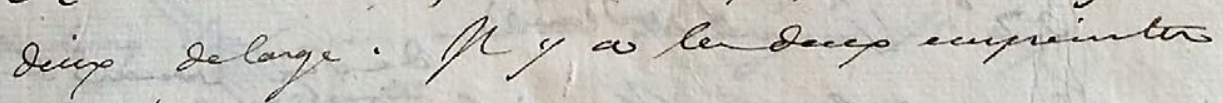
opposén.

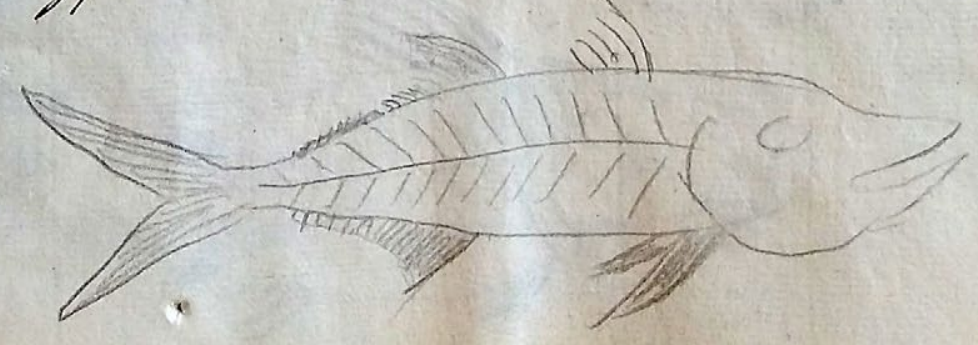

ANNEXE 2. - Suite; catalogue de Louis Augustin Guillaume Bosc, spécimens 58 à 62.

GEODIVERSITAS $\cdot 2019 \cdot 41(2)$

103 


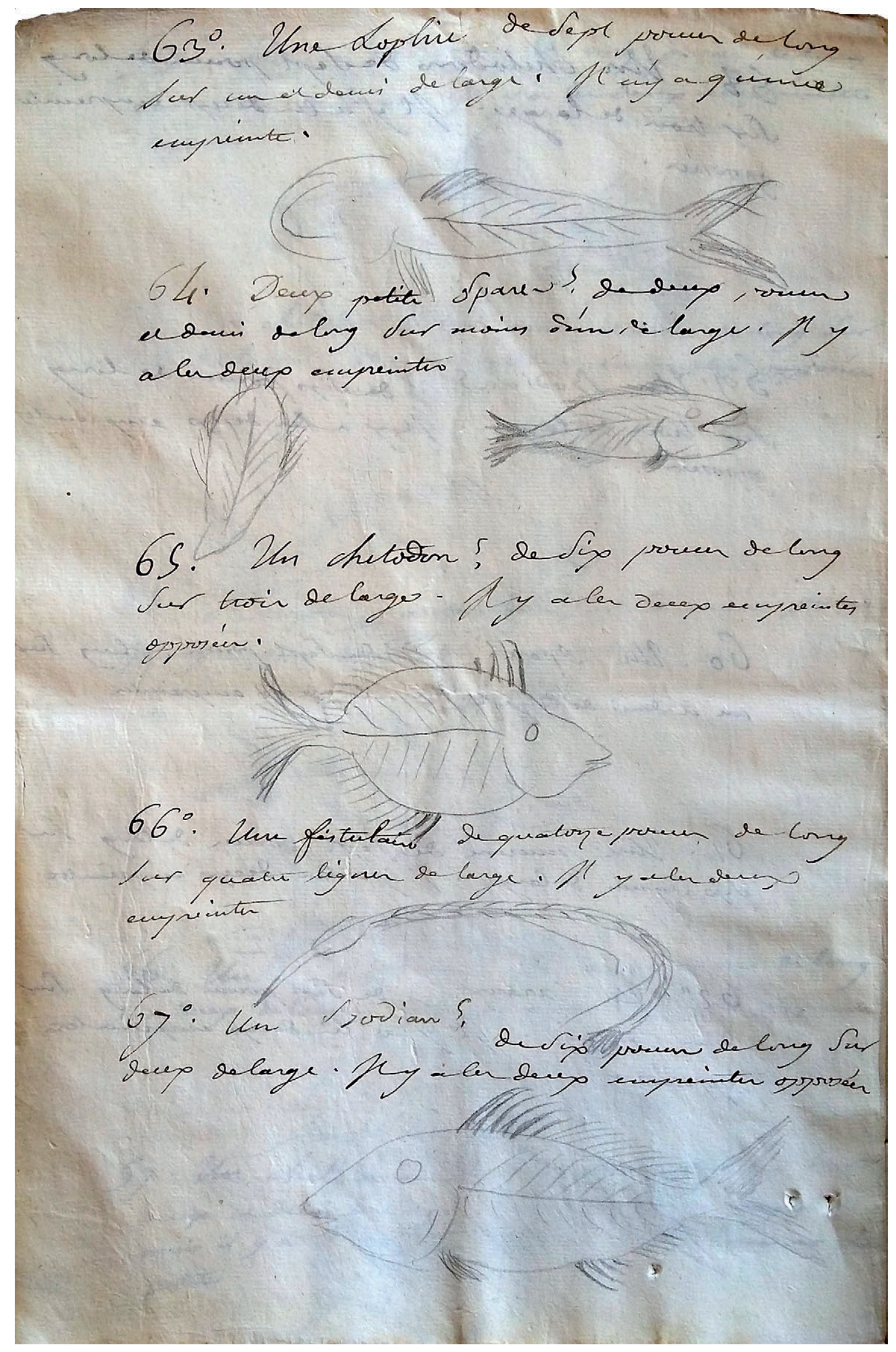

ANNEXE 2. - Suite; catalogue de Louis Augustin Guillaume Bosc, spécimens 63 à 67. 


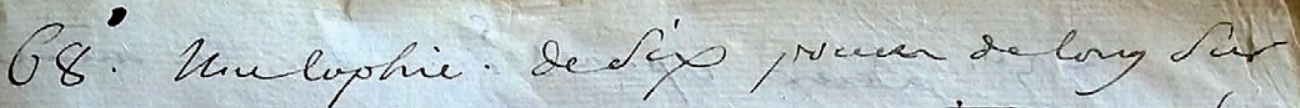

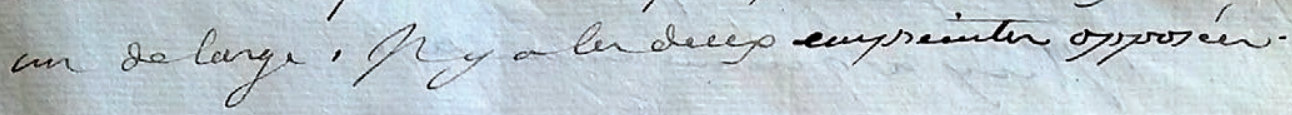

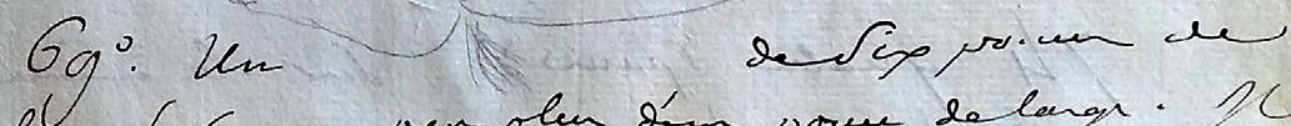

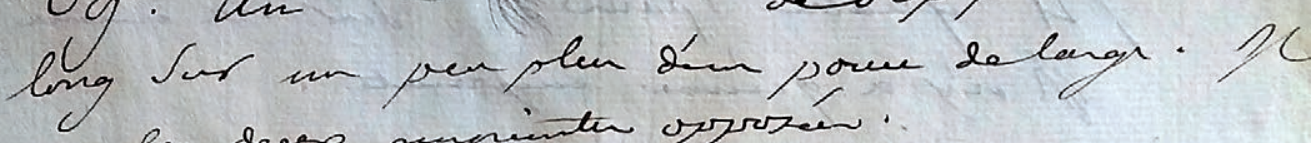

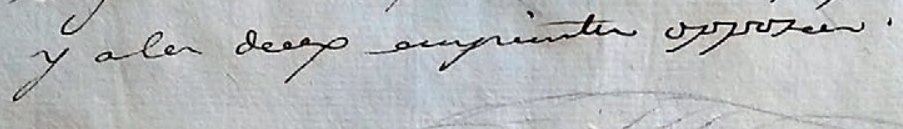

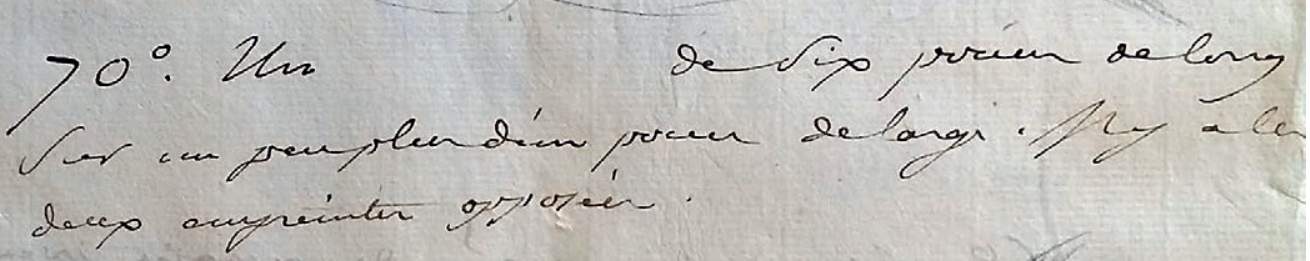

(7) क

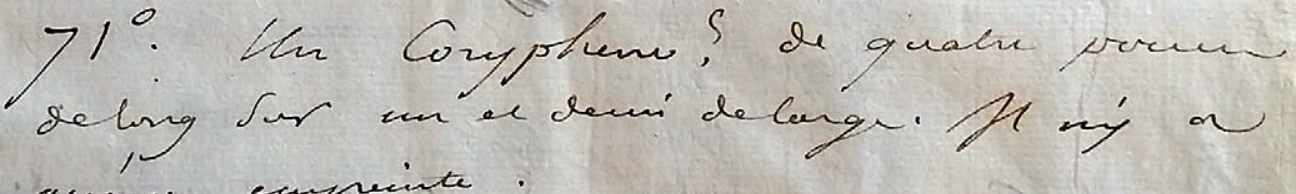
quem eayprexinte

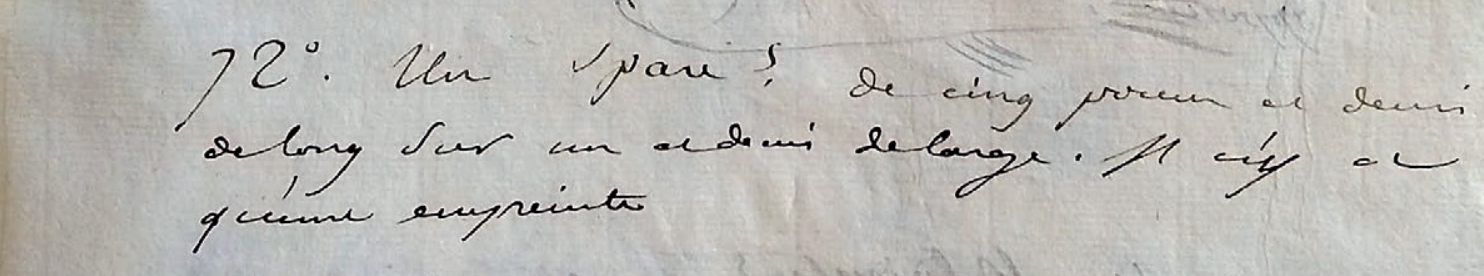
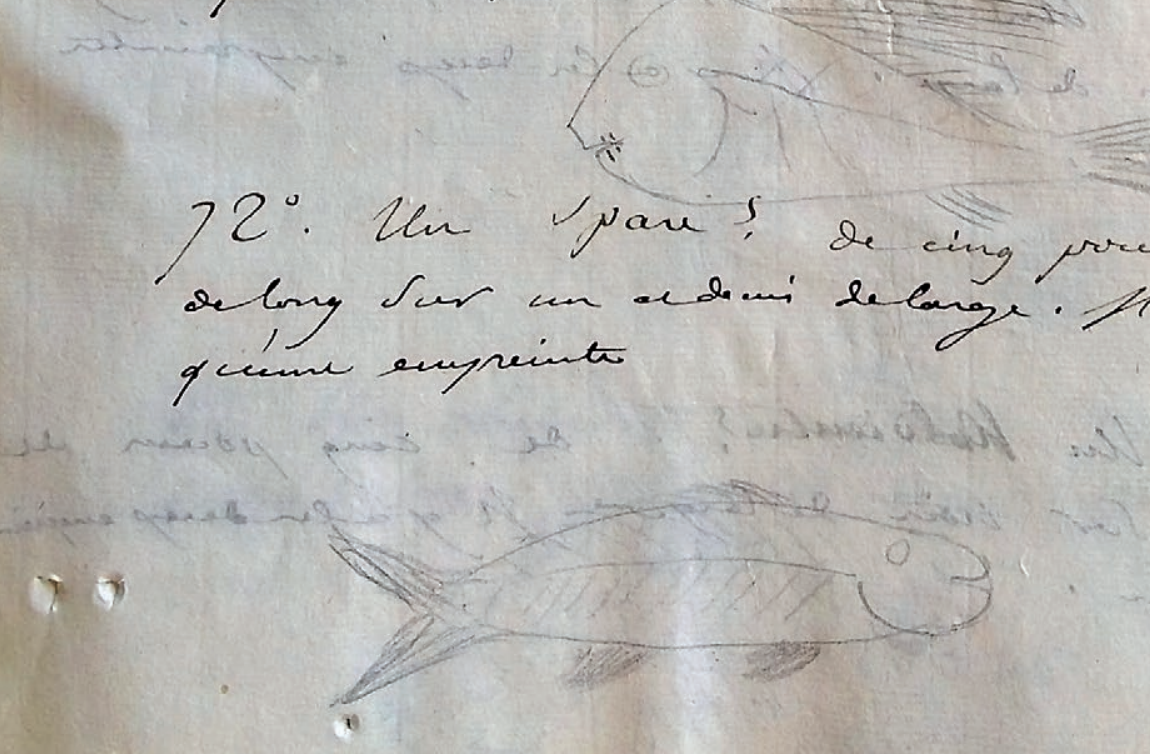

ANNEXE 2. - Suite; catalogue de Louis Augustin Guillaume Bosc, spécimens 68 à 72. 
$7^{0}$. Deve dpares S Sor ba neme prime. Mrly a qúane cupreinte.

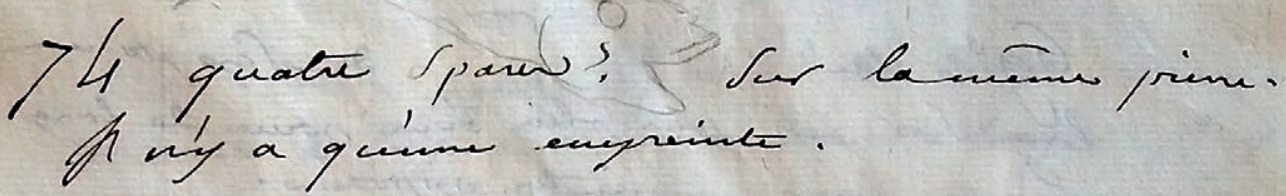

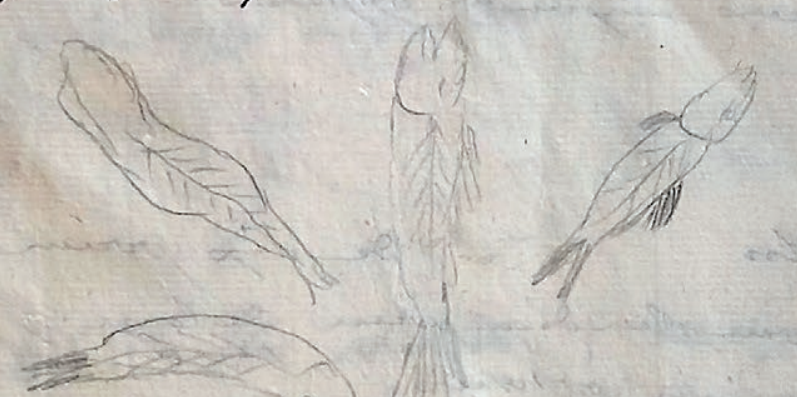

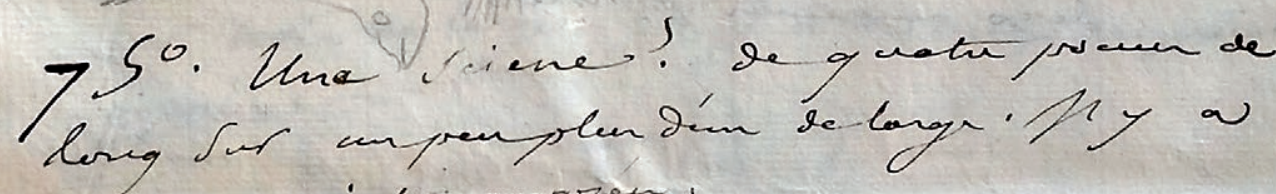
derep enyrimar opproin.

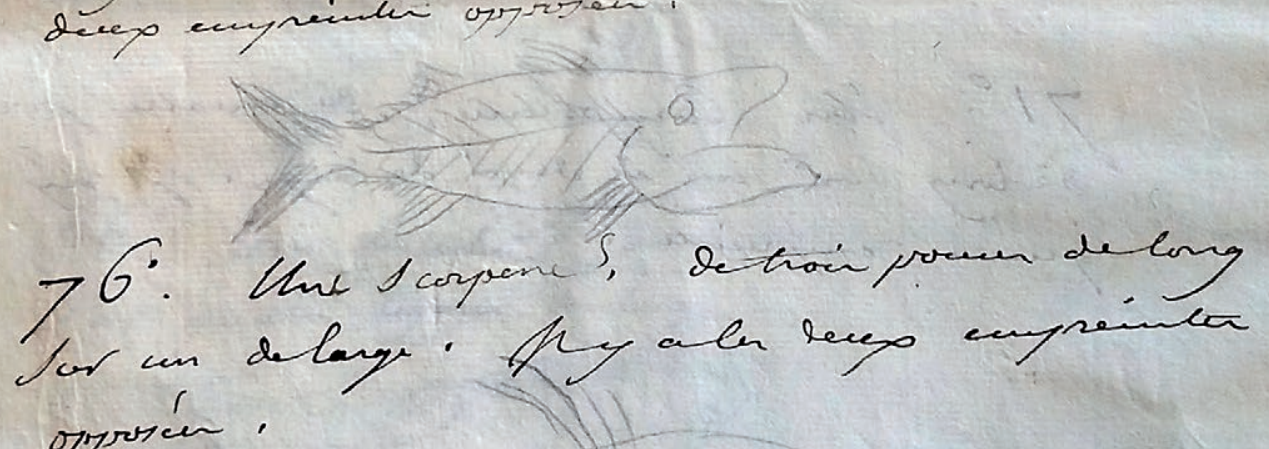
opprián.

$77^{\circ}$ He hbobiancus! de cing joum de

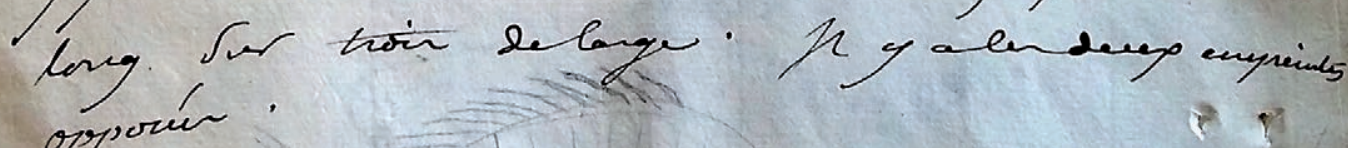

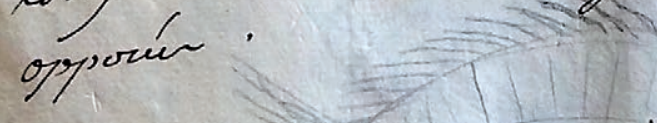

त)

ANNEXE 2. - Suite; catalogue de Louis Augustin Guillaume Bosc, spécimens 73 à 77. 


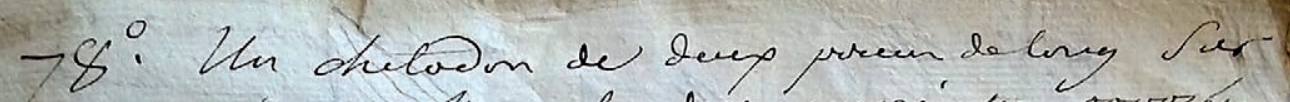

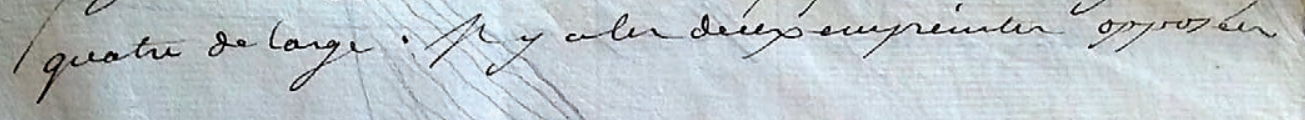

$79^{\circ}$ Une choobrenthe! de cing Sor dexp delange. Af y a len daeps

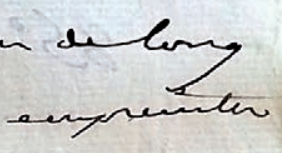
rporean

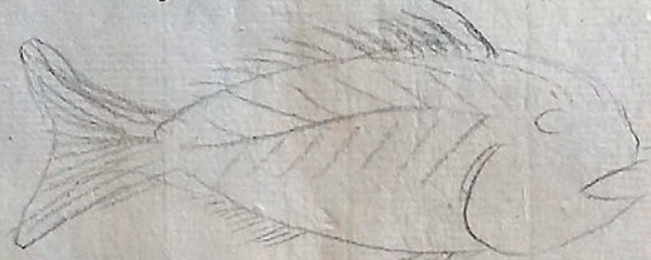

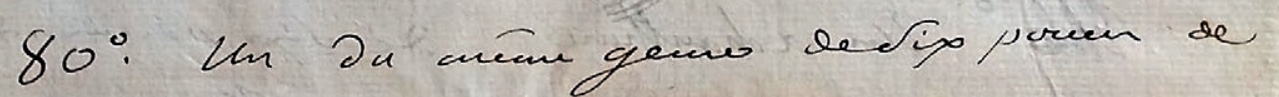

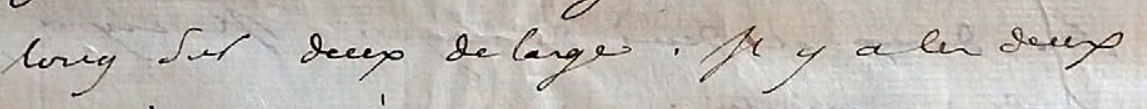
rapreinter opporeen.

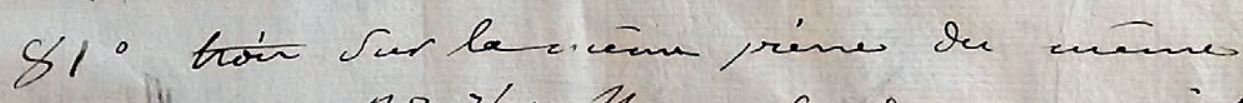

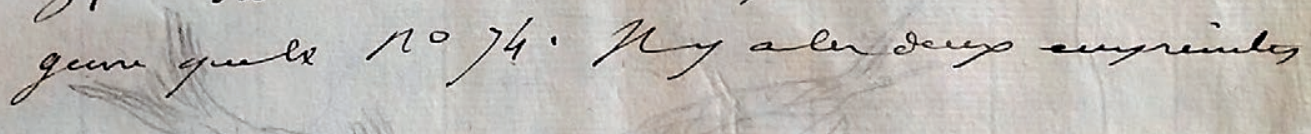
(ans (1) (1)

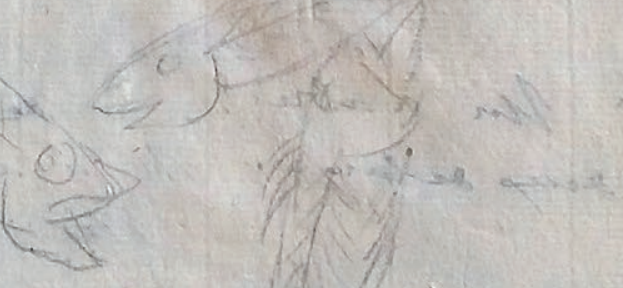

82. Un cheloton de cing sreen id long for

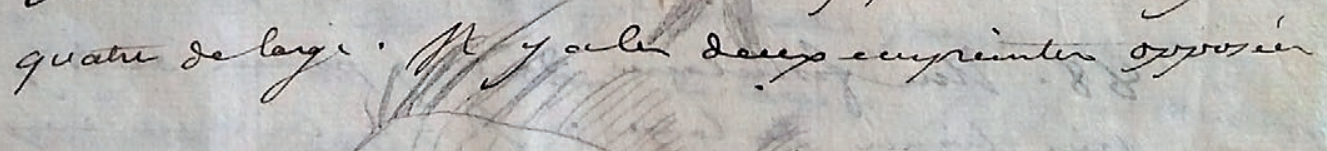


83: An
un relaye

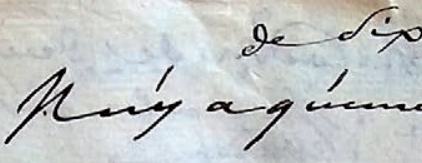

\$o/

reyprate.

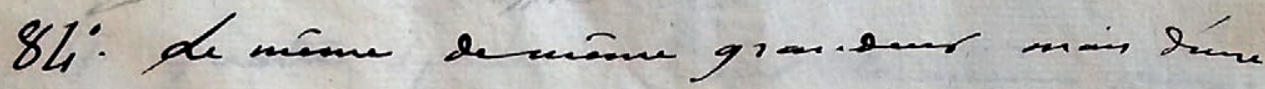

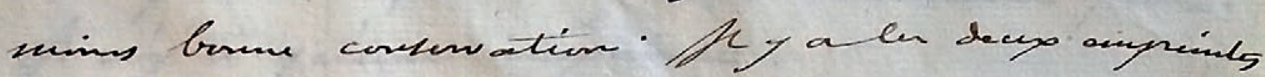
85. Un chetwom de so proun de cong far

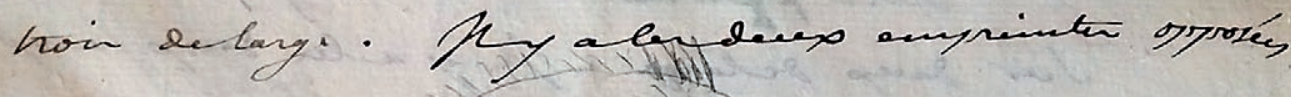
(1)

()

Q66: Un a chanthuro

dedio puam dicky

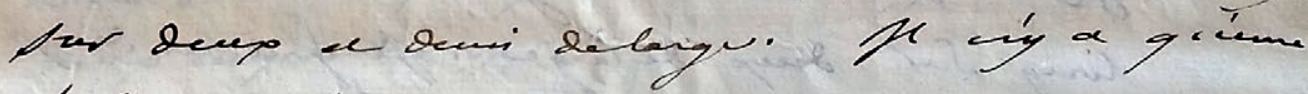
fade coppaina.

8.). Un Labre! de cing poun decay

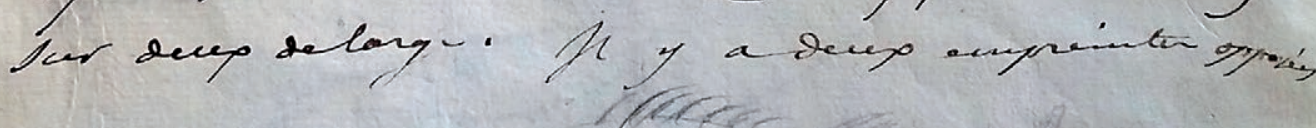

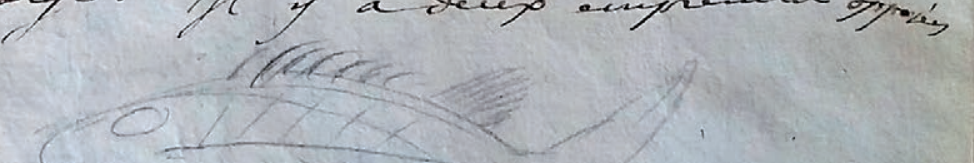

88. Une fiskdacie de sing porcen debing Sor vion liguen de laye. hy a bi deces cuppintar ग)7roin.

ANNEXE 2. - Suite; catalogue de Louis Augustin Guillaume Bosc, spécimens 83 à 88. 


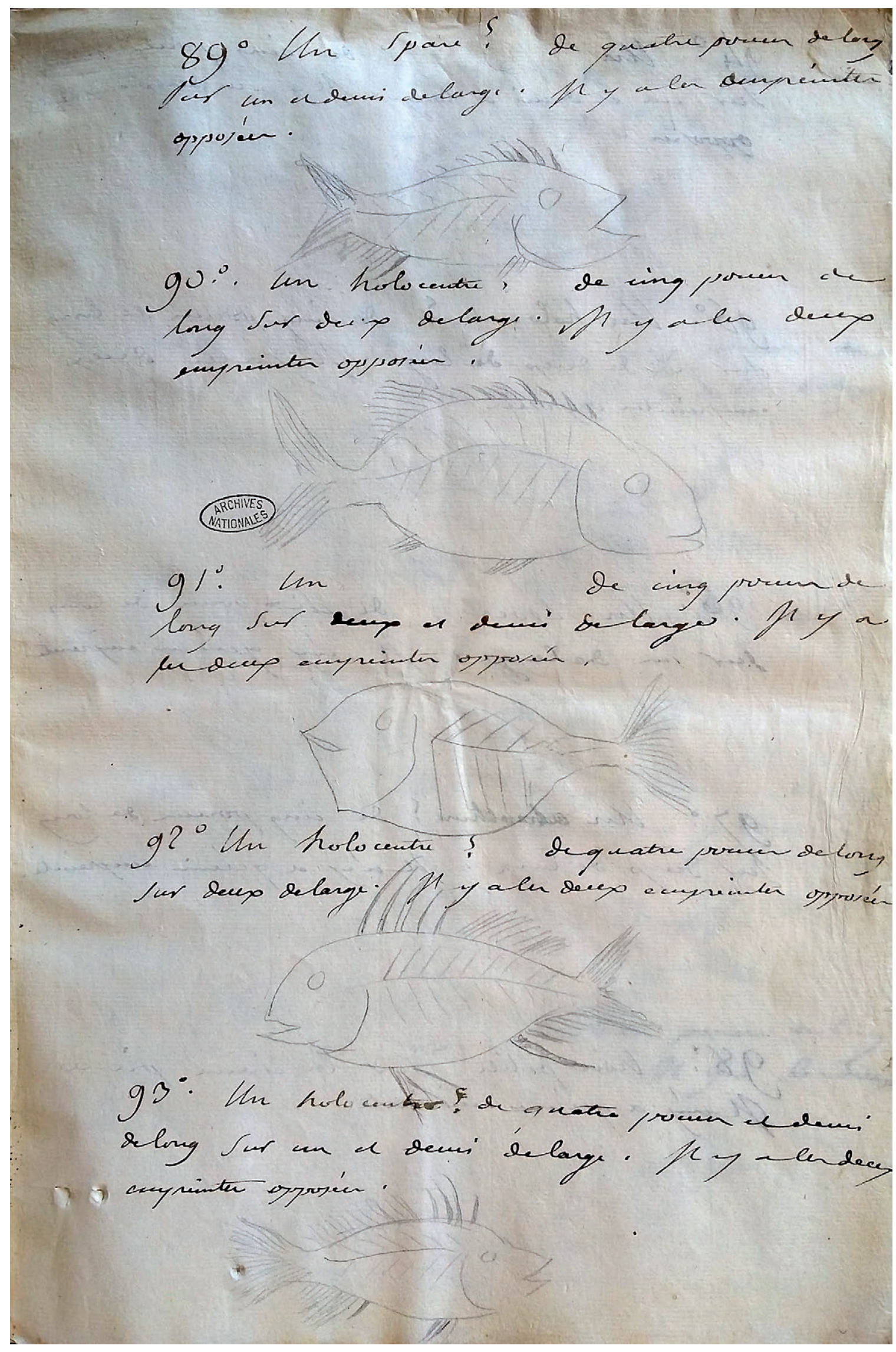

ANNEXE 2. - Suite; catalogue de Louis Augustin Guillaume Bosc, spécimens 89 à 93. 


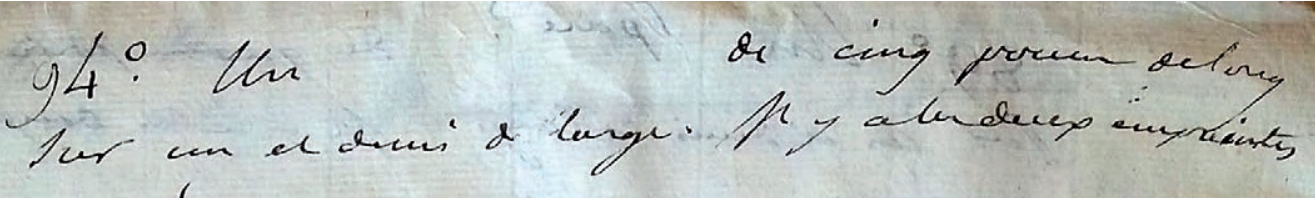
opporien.

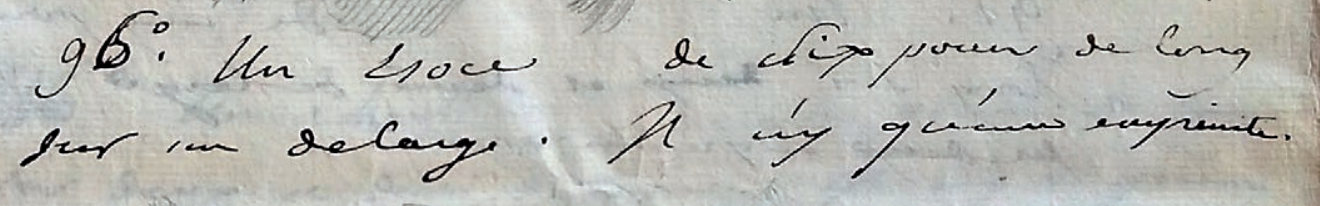

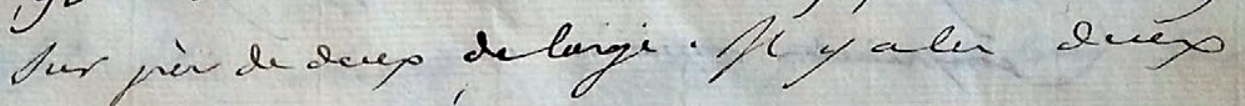
engreinter opporsen.

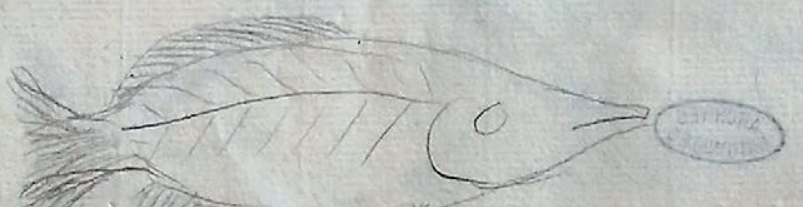

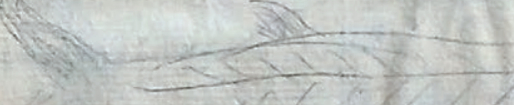

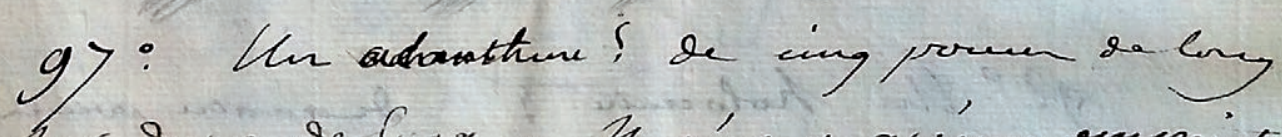

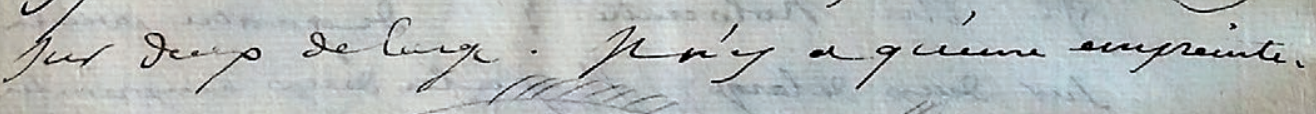

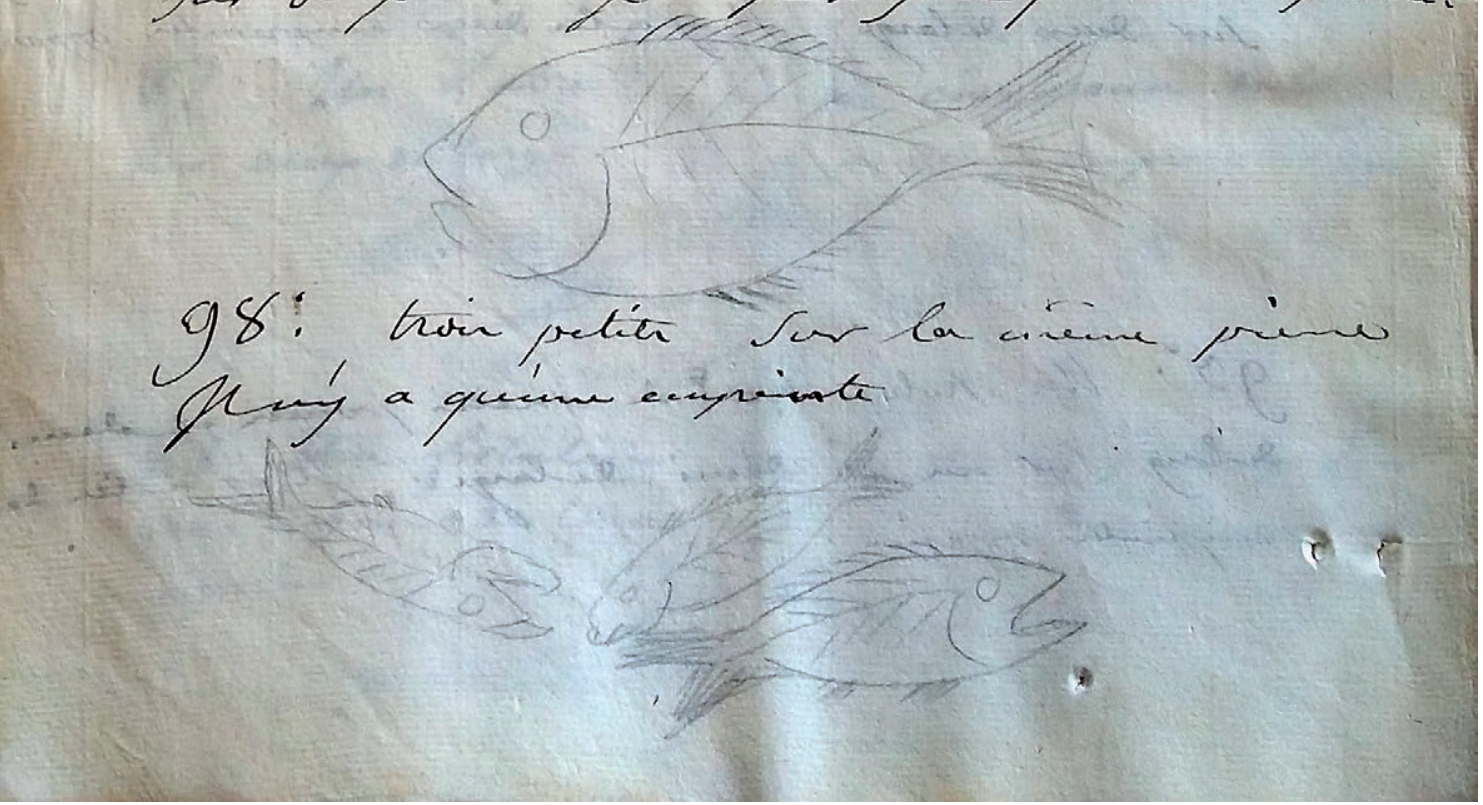

ANNEXE 2. - Suite; catalogue de Louis Augustin Guillaume Bosc, spécimens 94 à 98. 
Les conditions d'acquisition de la collection Gazola par le Muséum national d'Histoire naturelle

99. Une Sciene? de cing prom de long for derep de large. Ily a kn dengo cupreinta opprosán.

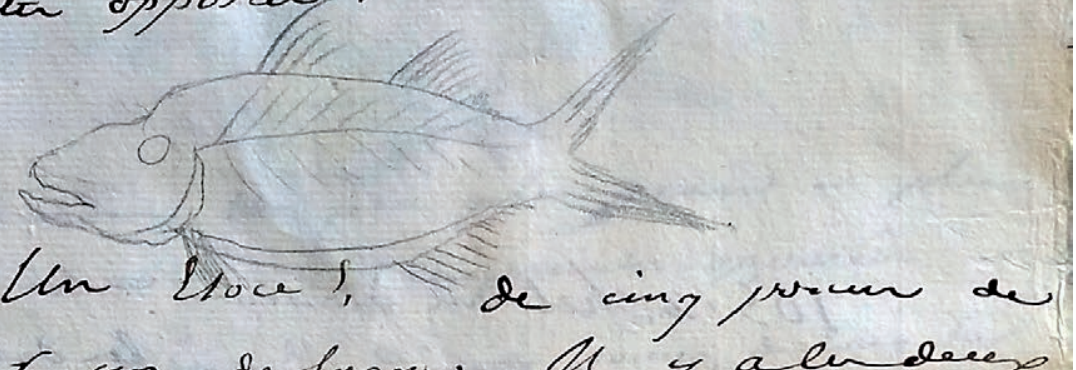

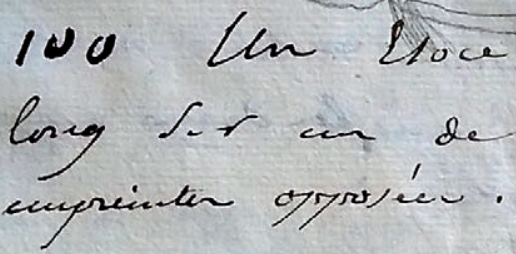

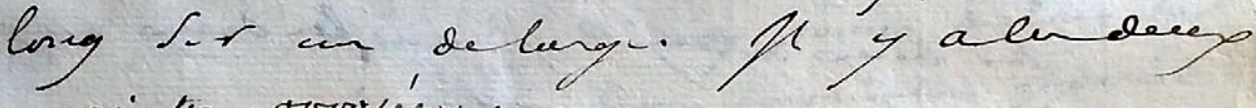

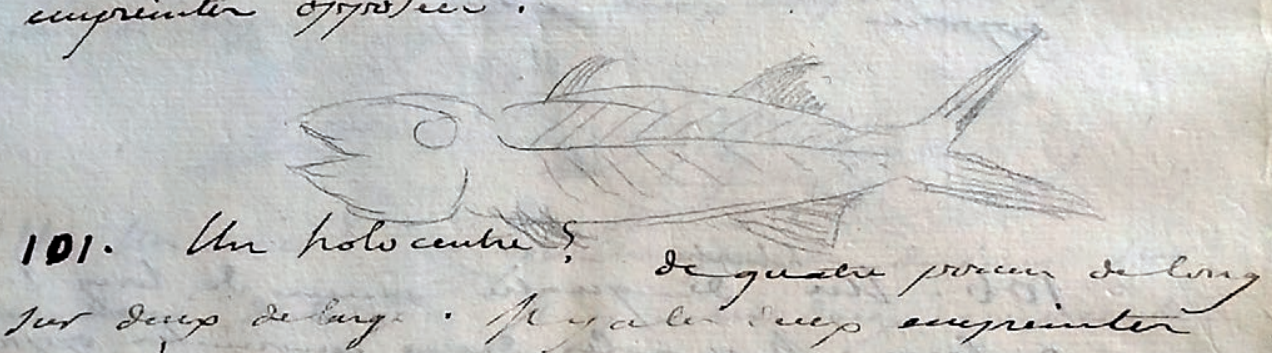
oporín.

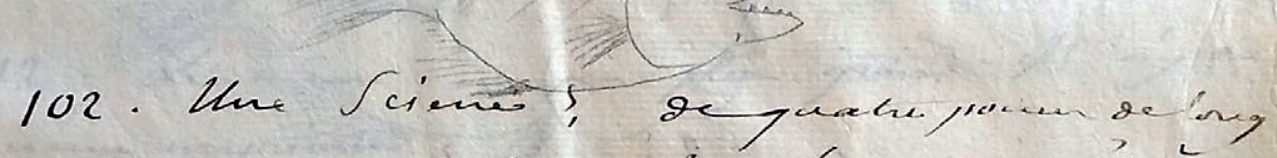

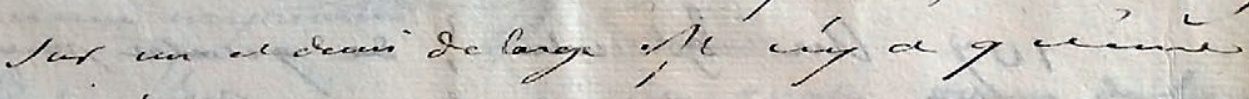
raprecice.

103.240

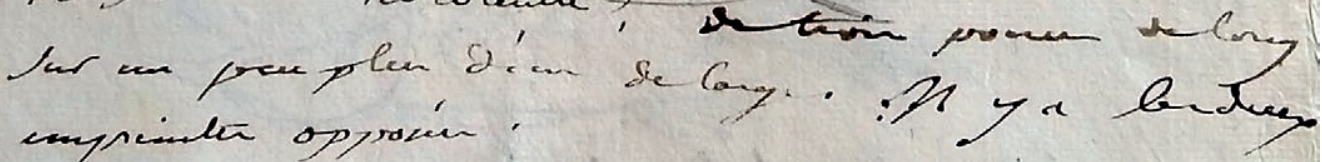
coppesile opprón.

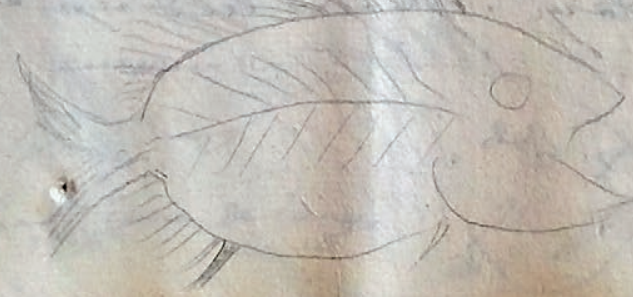

ANNEXE 2. - Suite; catalogue de Louis Augustin Guillaume Bosc, spécimens 99 à 103.

GEODIVERSITAS $\cdot 2019 \cdot 41(2)$

111 
- Brignon A.

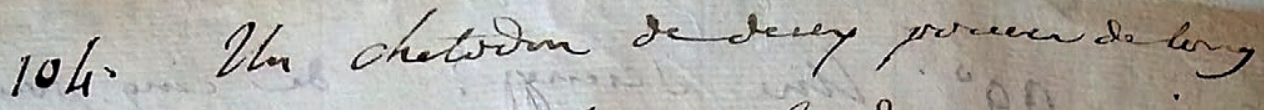

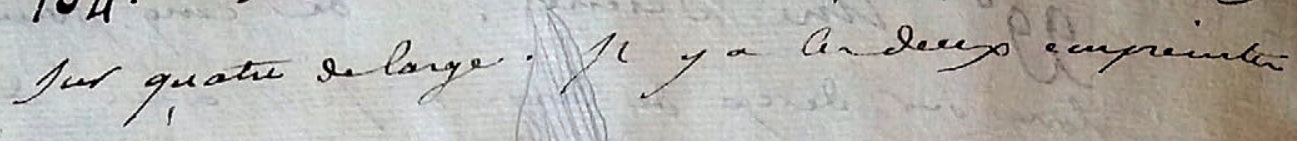
गyporear.

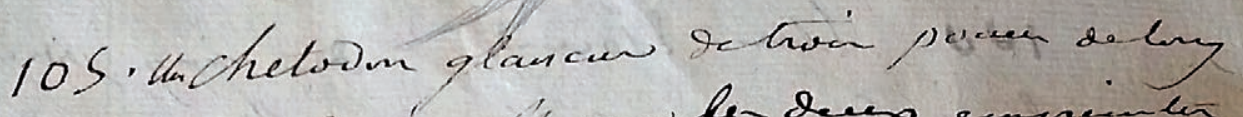

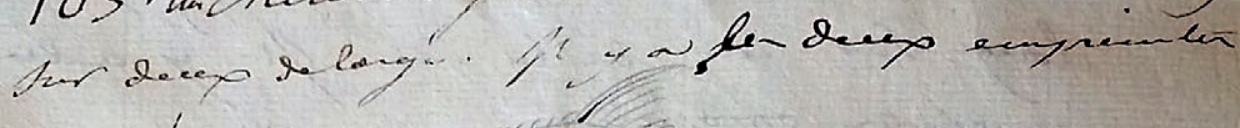
oproín.

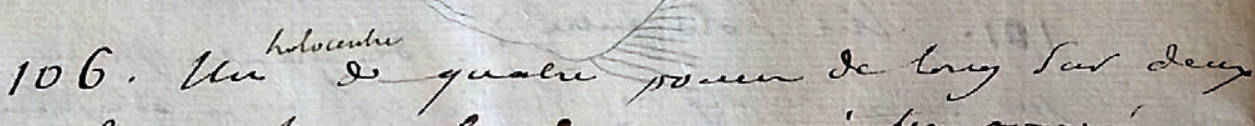

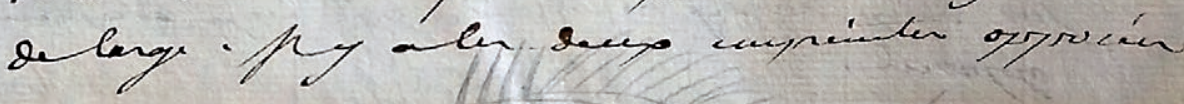

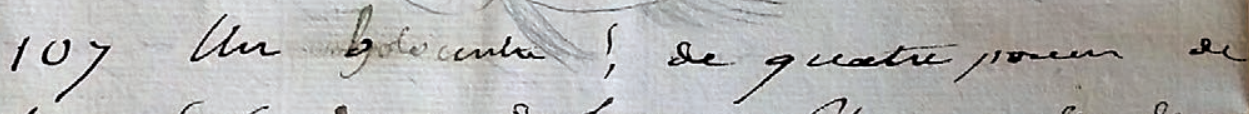

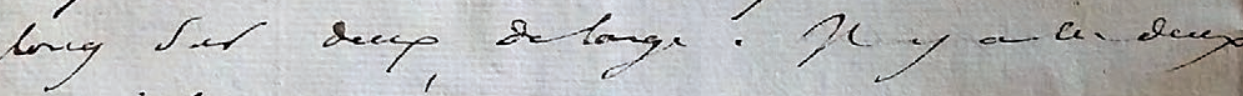

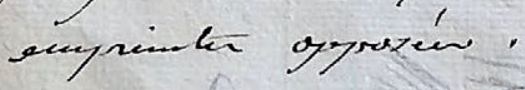

108. Un holocentre , de quahe procen de ling

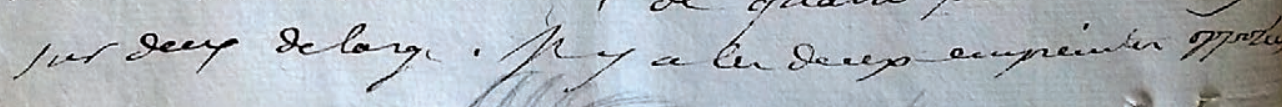

ANNEXE 2. - Suite; catalogue de Louis Augustin Guillaume Bosc, spécimens 104 à 108.

112

GEODIVERSITAS $\cdot 2019 \cdot 41(2)$ 


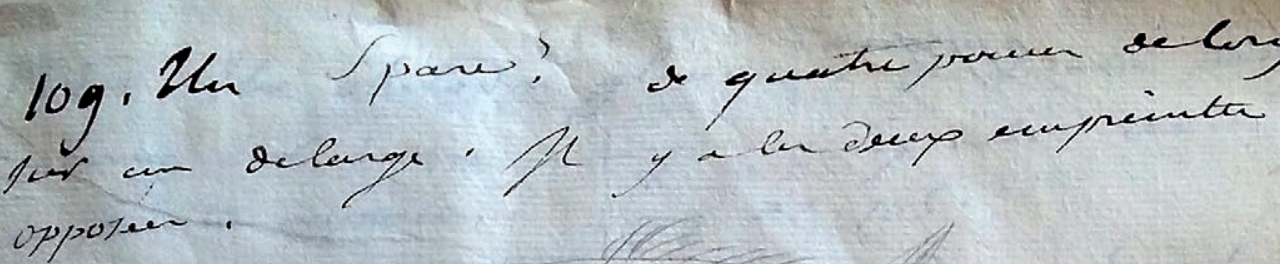

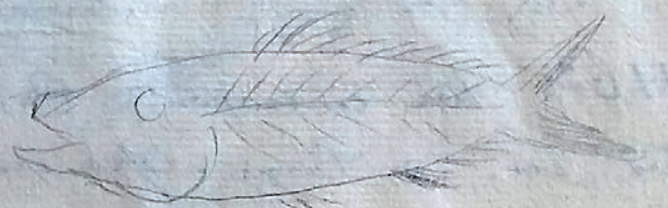

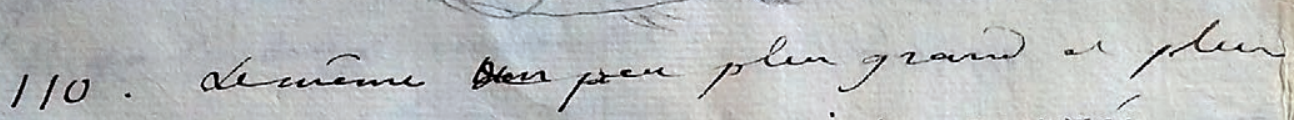

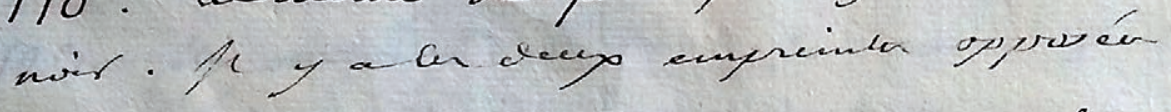

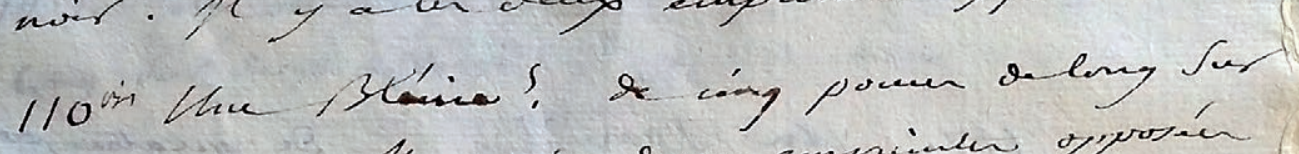

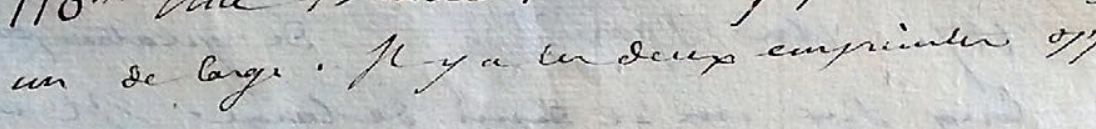

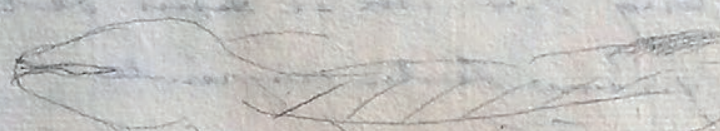

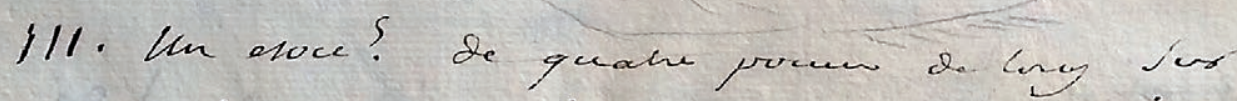

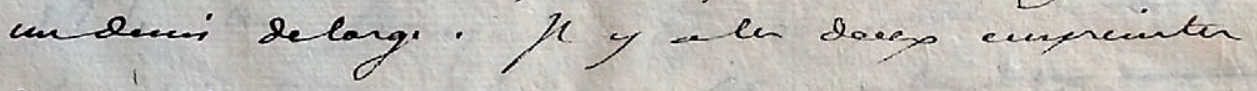
opprosen,

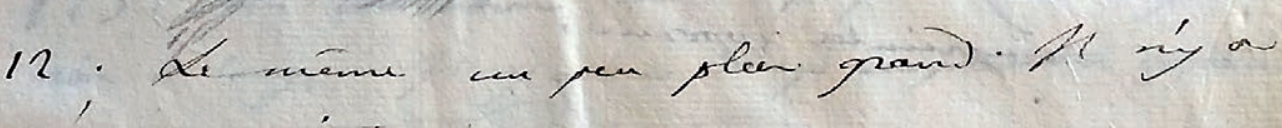

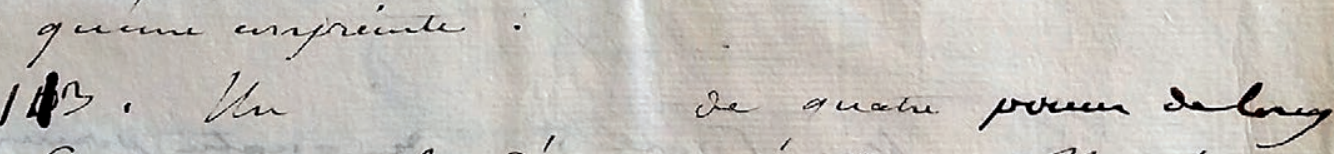

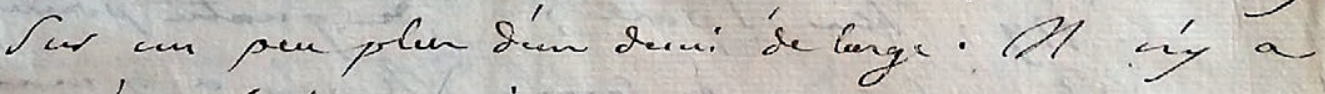
quime teub compraite.

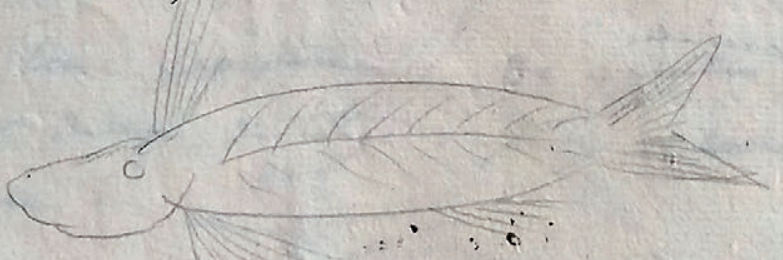

114; de ninue que de $4: 112$. Mry a 7) Yyceme expreinte.
$115 \cdot 3 \mathrm{~m}$
de ing fat cen
Se tang.
It a lad dens
exprein $c$ 


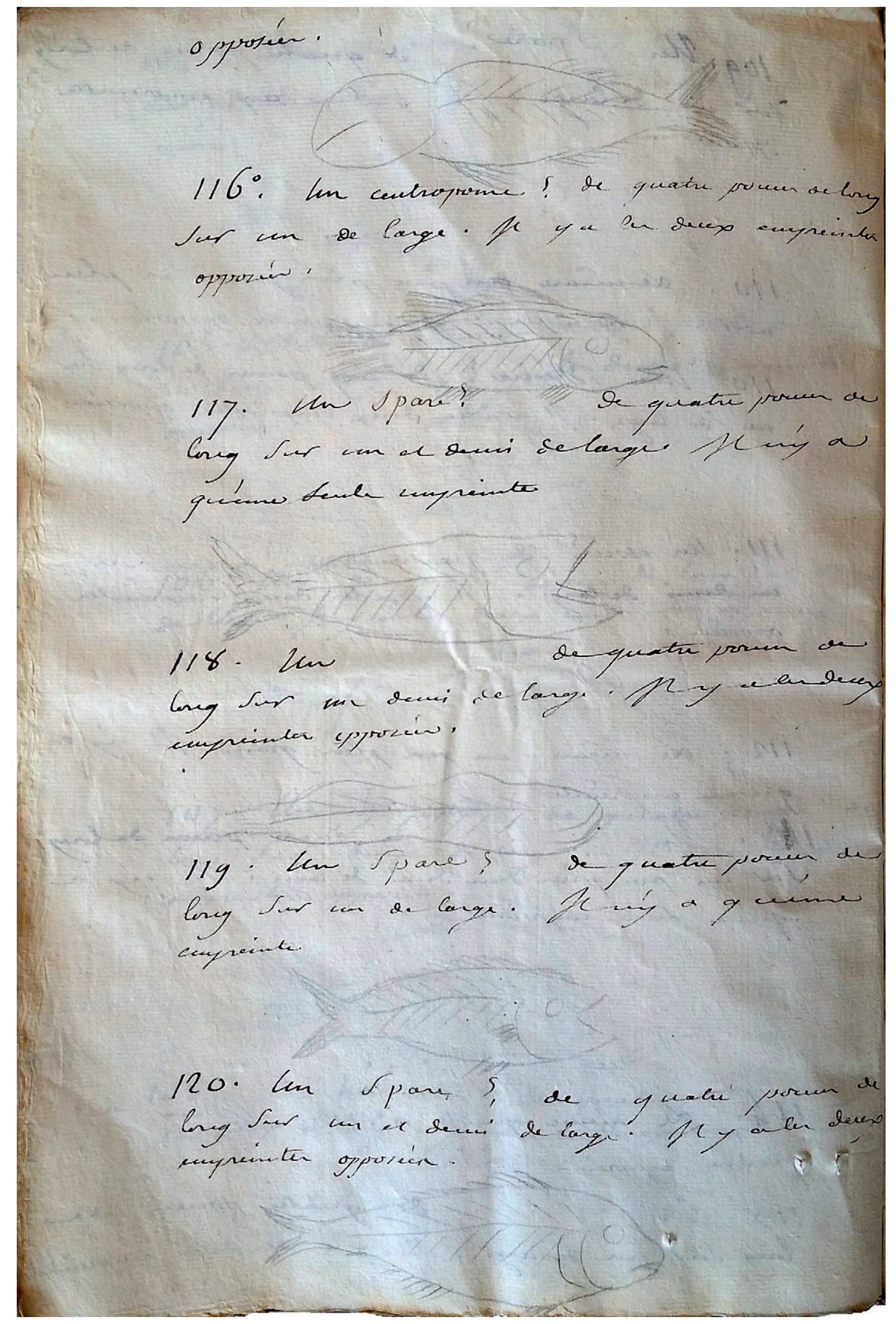

ANNEXE 2. - Suite; catalogue de Louis Augustin Guillaume Bosc, spécimens 116 à 120. 
Les conditions d'acquisition de la collection Gazola par le Muséum national d'Histoire naturelle

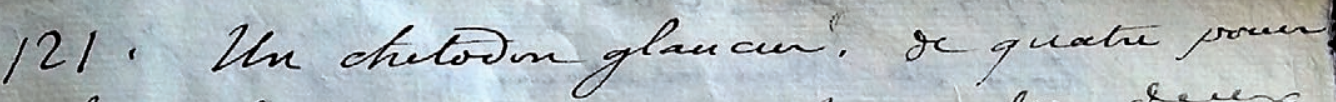

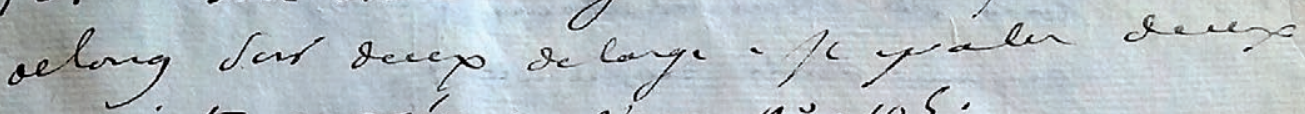
empreinta opjorén. Vizy n'.105.

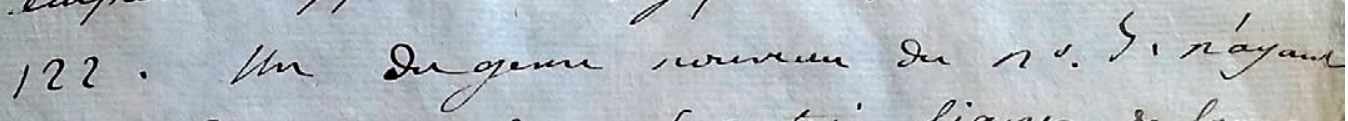
que is prom do buy for hoin lignen de lange. It yabrdery expreinta.

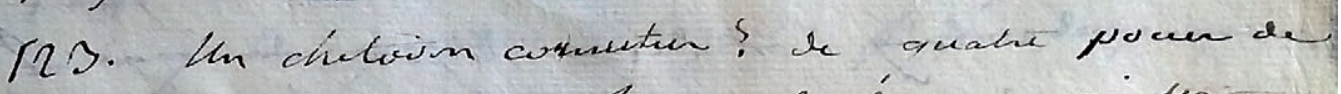

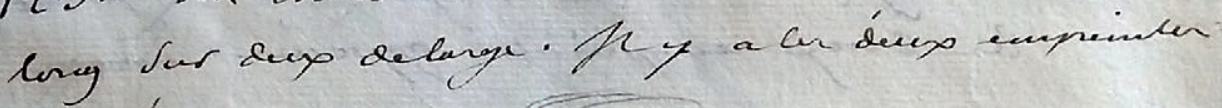
oprorión.

124 un Fegajes dehoir prowen de longes

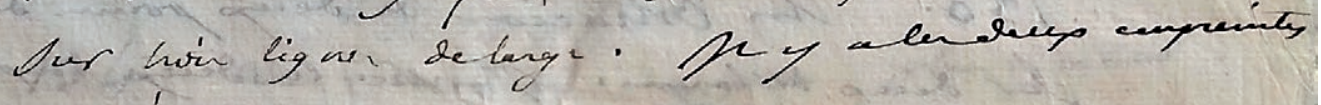
opjosian.

215. Un chataren de becp proen delang ín

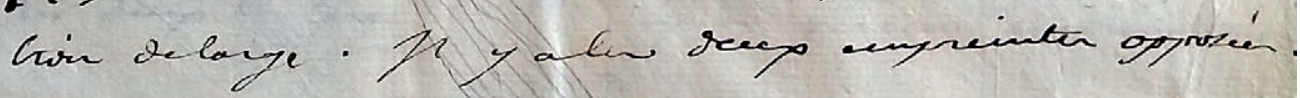

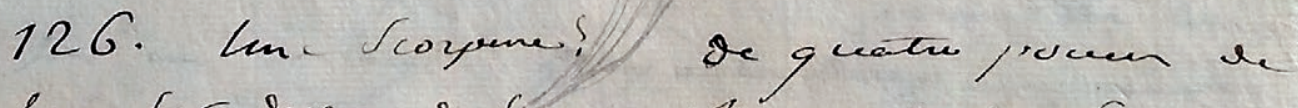

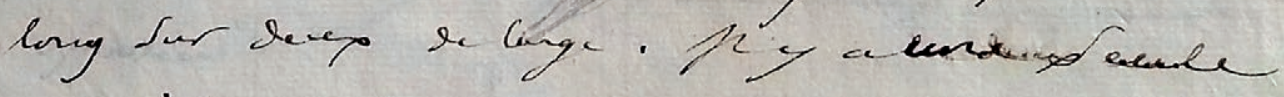
eapreinte.

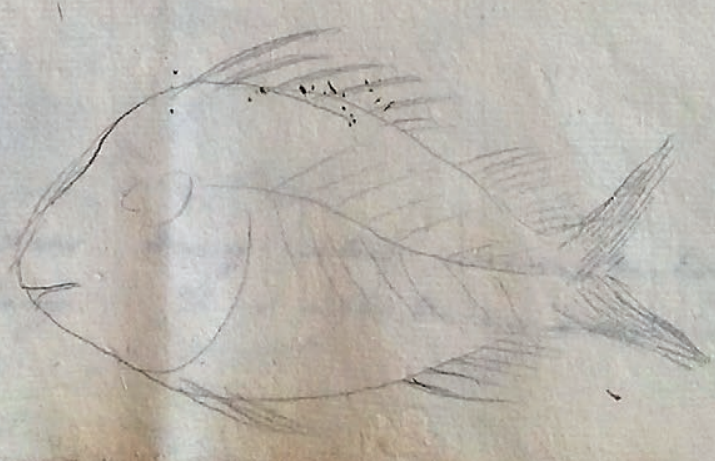

ANNEXE 2. - Suite; catalogue de Louis Augustin Guillaume Bosc, spécimens 121 à 126.

GEODIVERSITAS $\cdot 2019 \cdot 41(2)$

115 


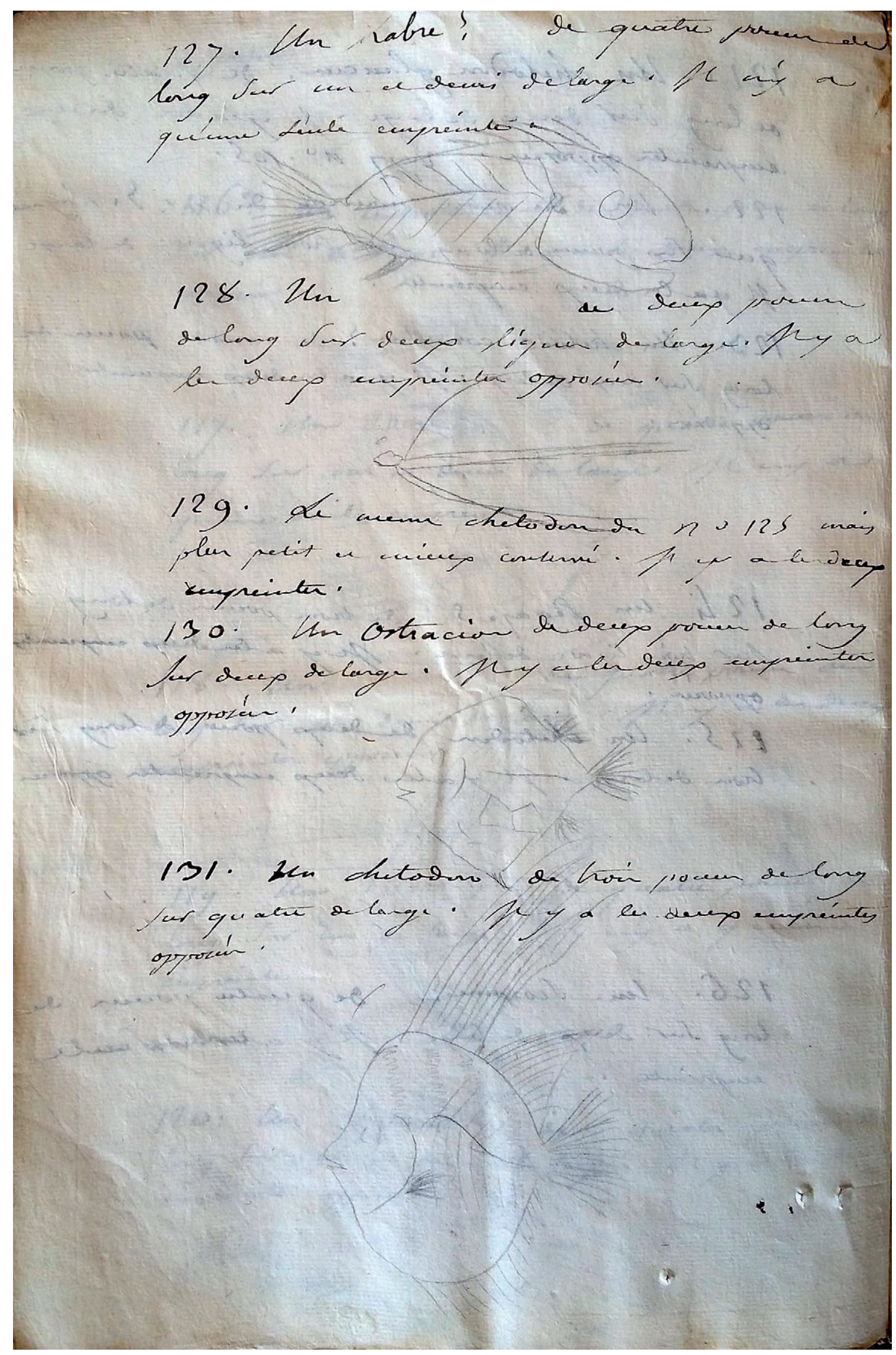

ANNEXE 2. - Suite; catalogue de Louis Augustin Guillaume Bosc, spécimens 127 à 131. 


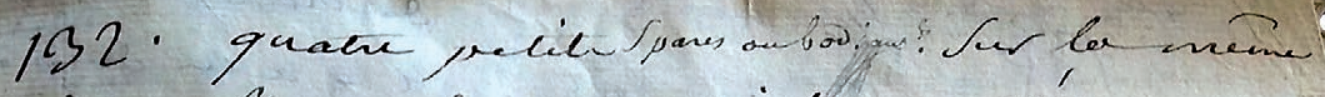

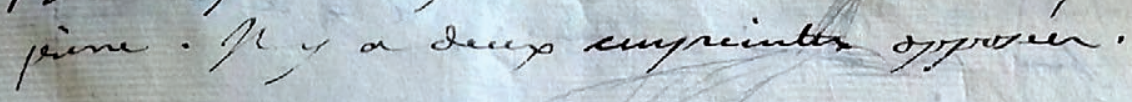

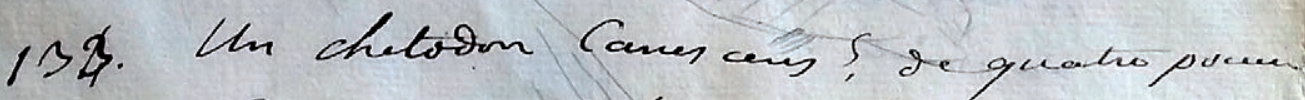

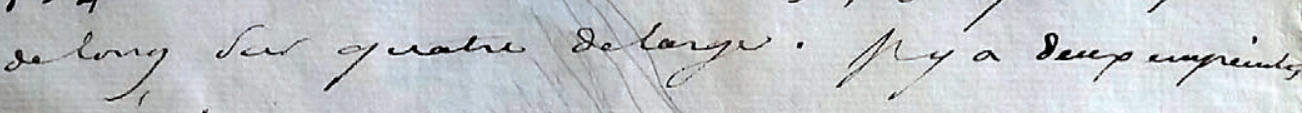
opprozen

134. de newn an pen plen previt.

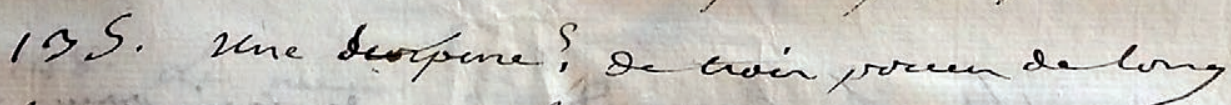

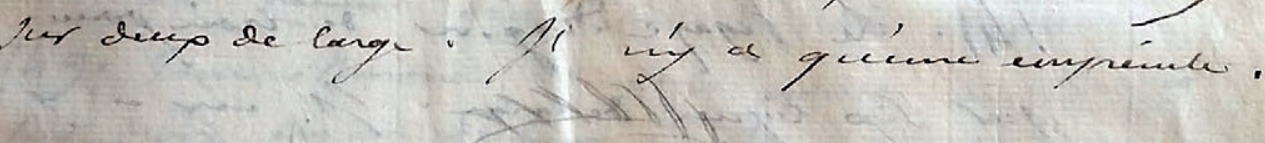
$136.2 \mathrm{n}$ pare?

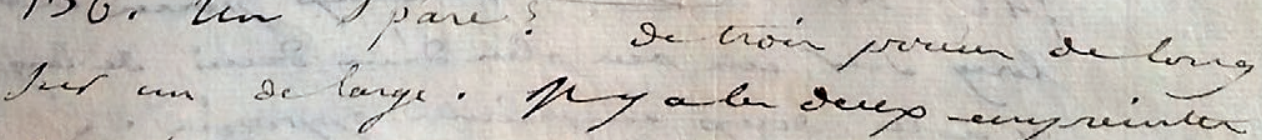
gyosín

7 का

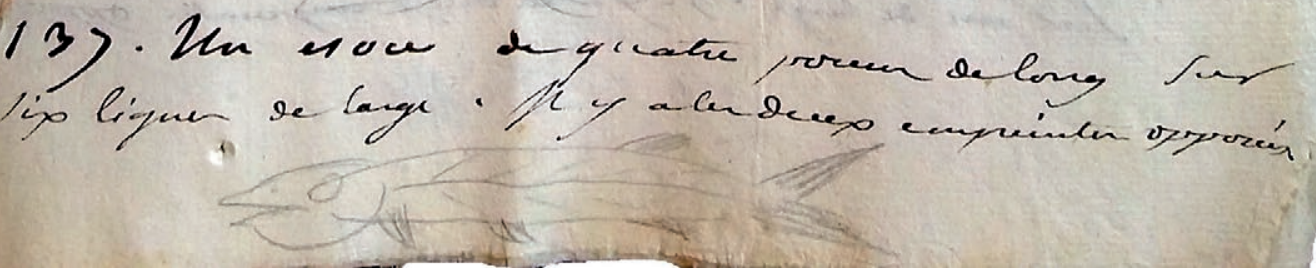

ANNEXE 2. - Suite; catalogue de Louis Augustin Guillaume Bosc, spécimens 132 à 137. 


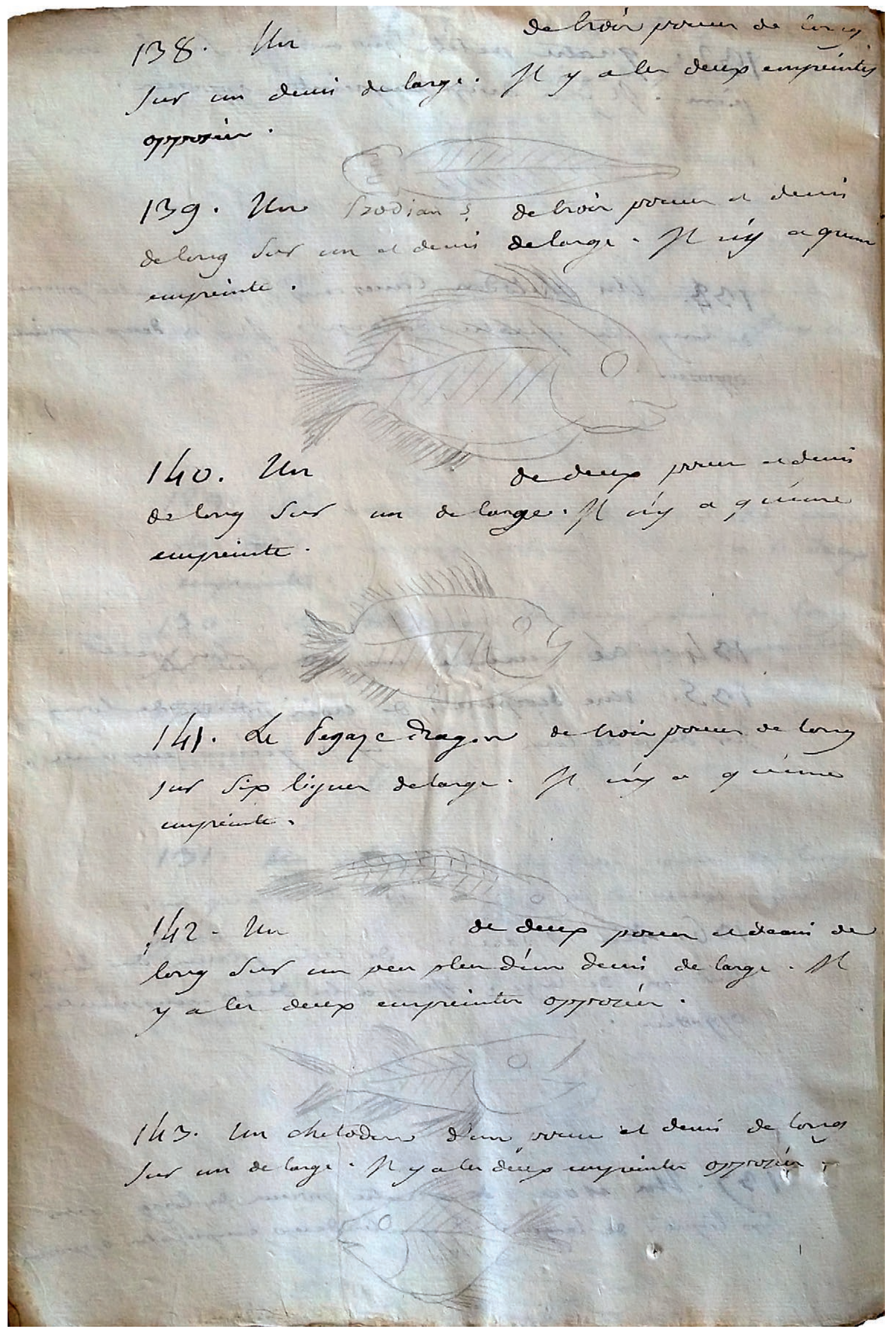

ANNEXE 2. - Suite; catalogue de Louis Augustin Guillaume Bosc, spécimens 138 à 143. 


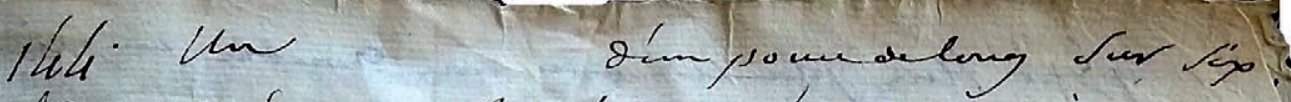

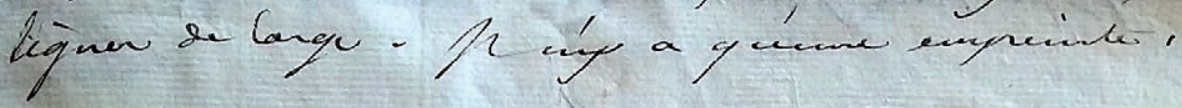

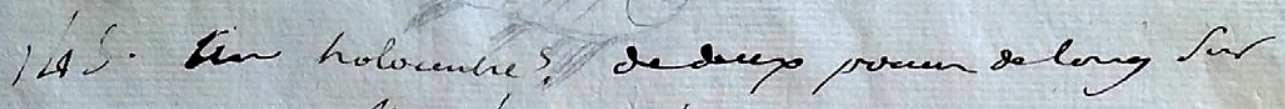

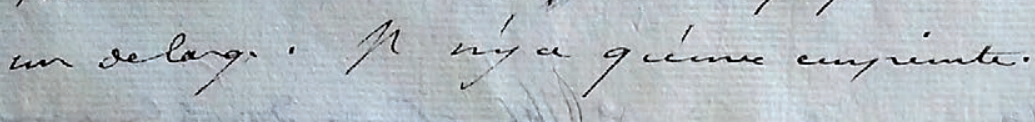

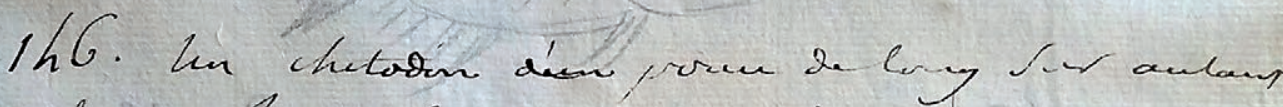

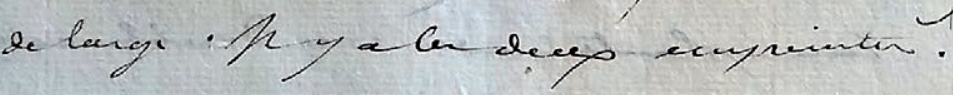

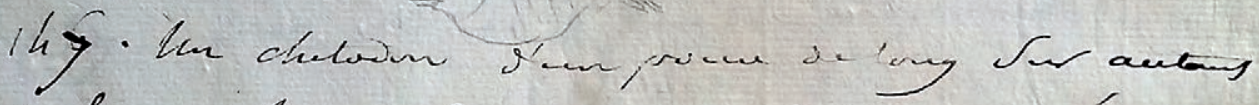
$\sum_{0}^{0}+1+2$

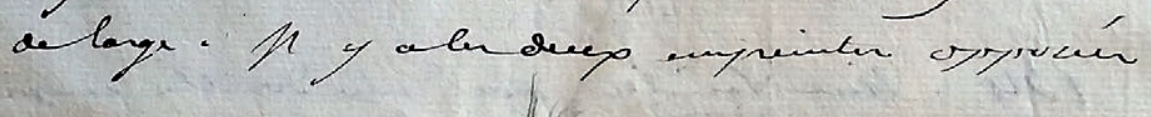

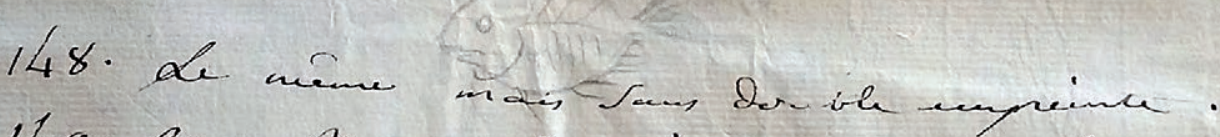

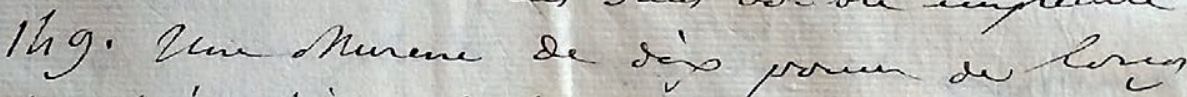

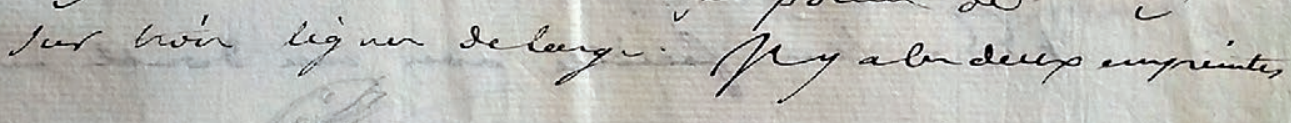

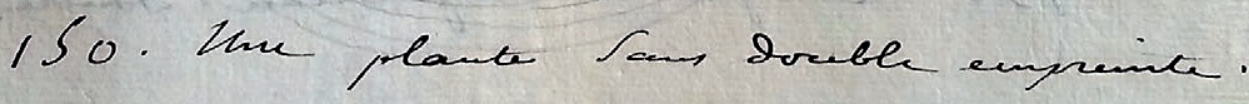
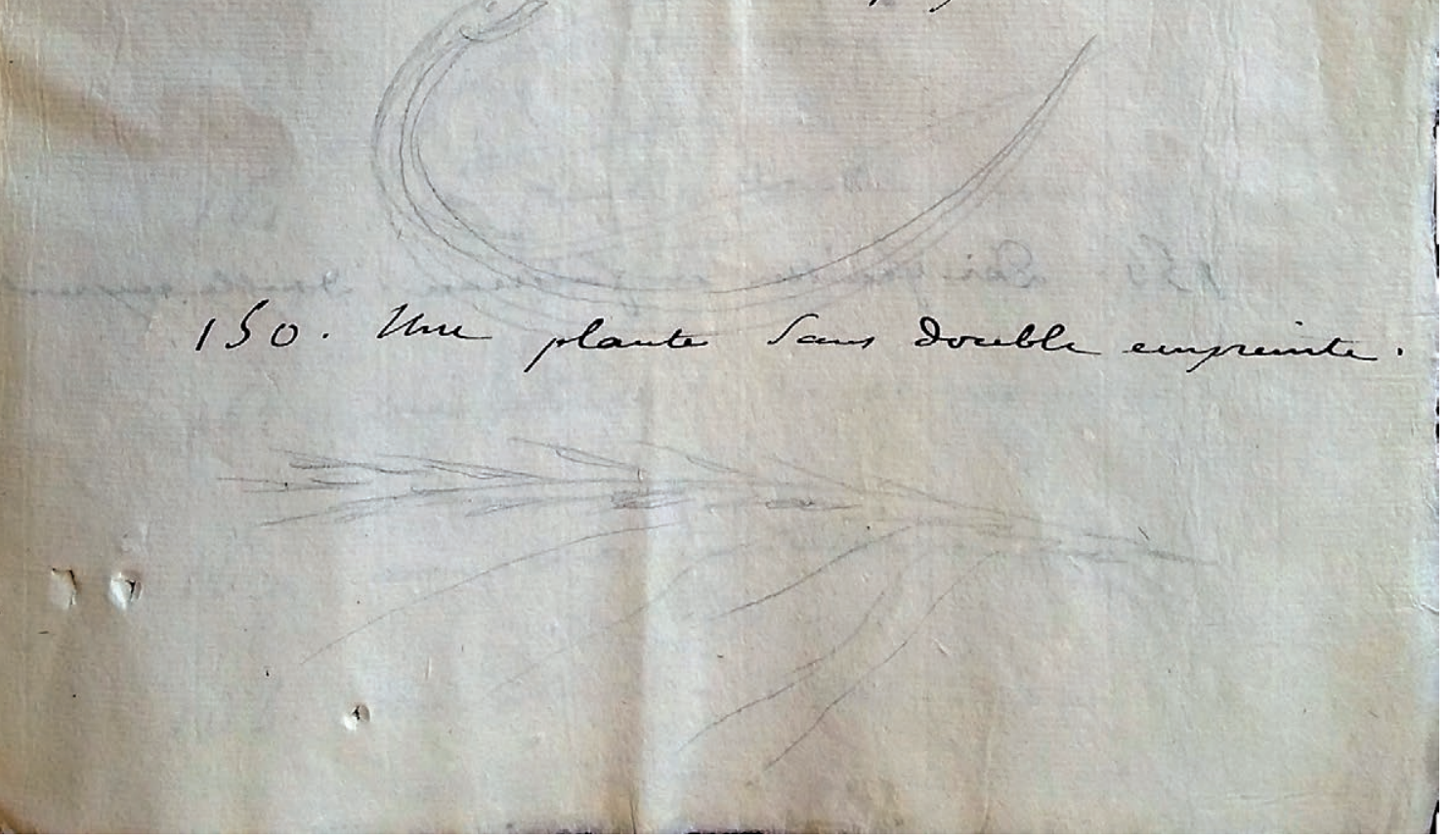

ANNEXE 2. - Suite; catalogue de Louis Augustin Guillaume Bosc, spécimens 144 à 150. 


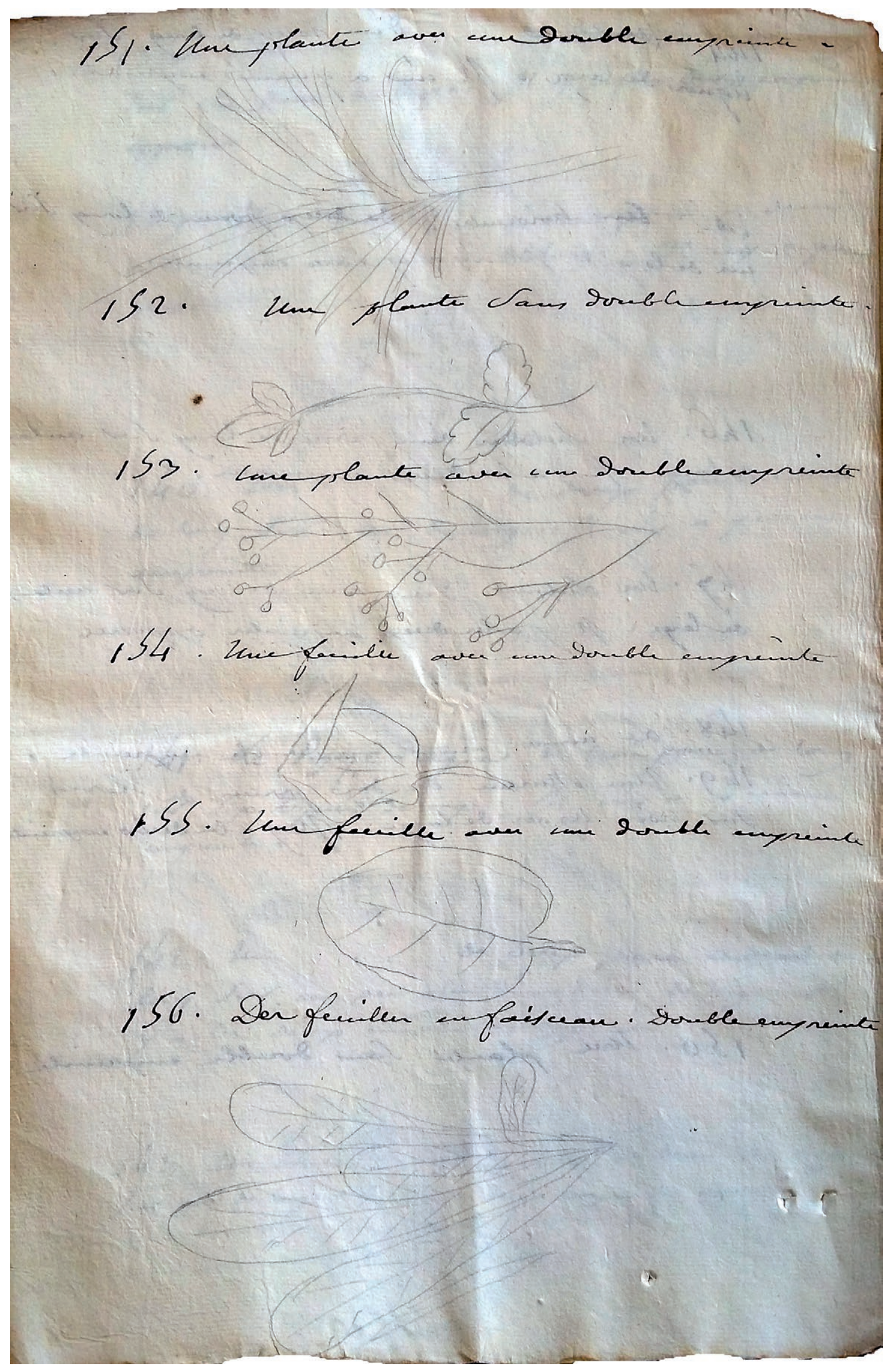

ANNEXE 2. - Suite; catalogue de Louis Augustin Guillaume Bosc, spécimens 151 à 156. 


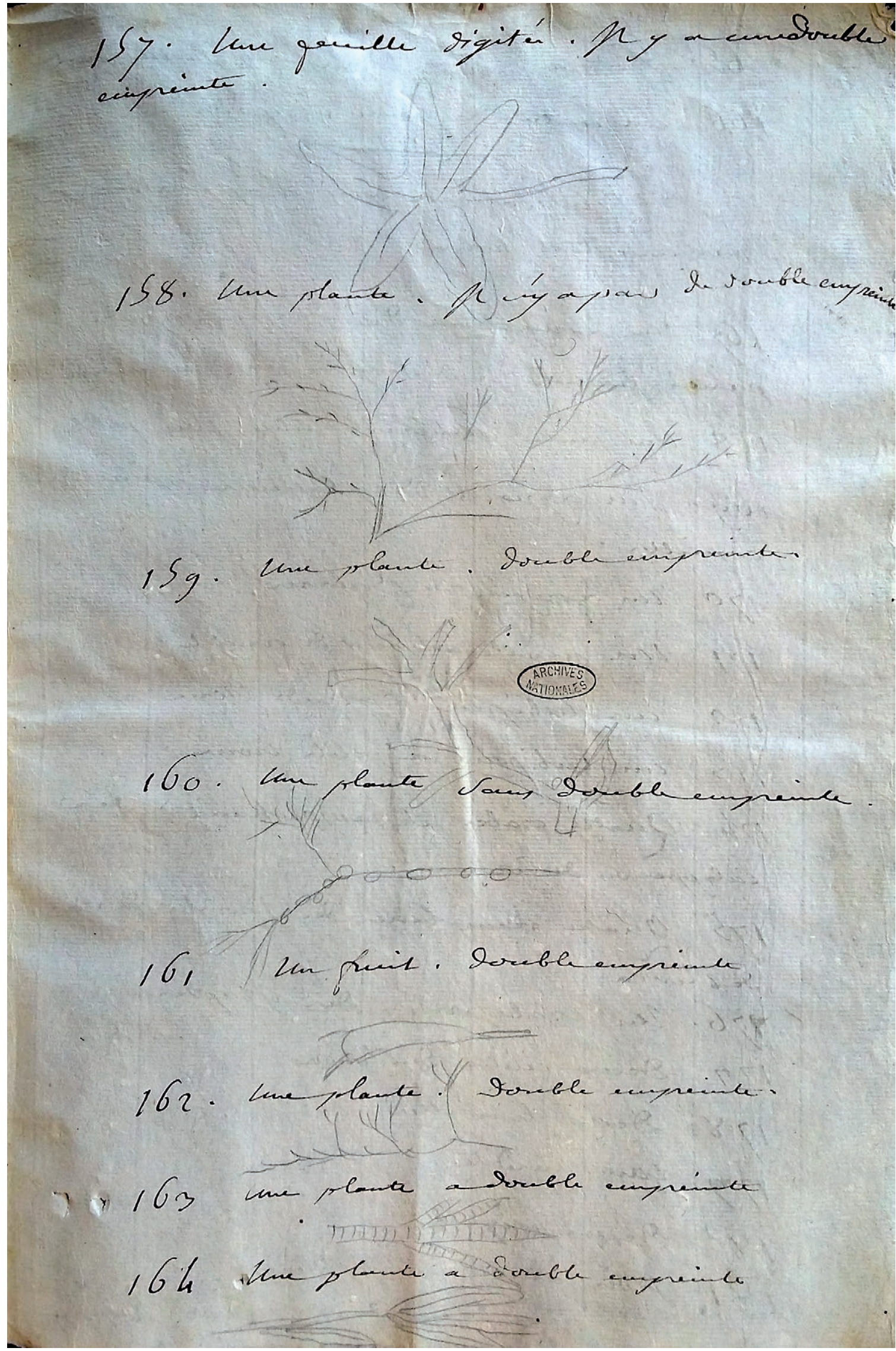

ANNEXE 2. - Suite; catalogue de Louis Augustin Guillaume Bosc, spécimens 157 à 164. 


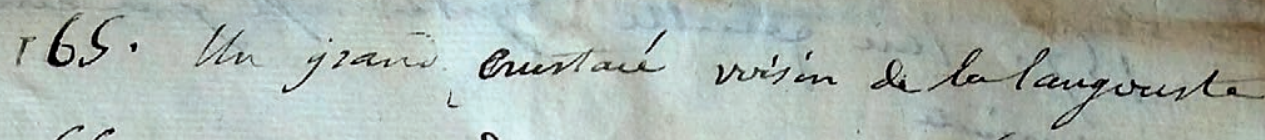
166 cm acere he gann occipode!

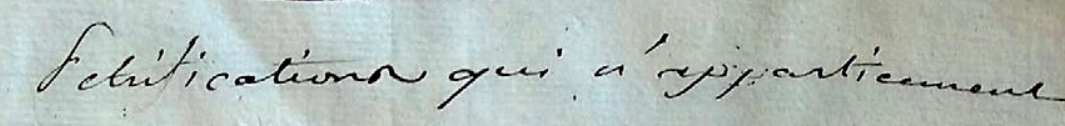
par and Mut bolca

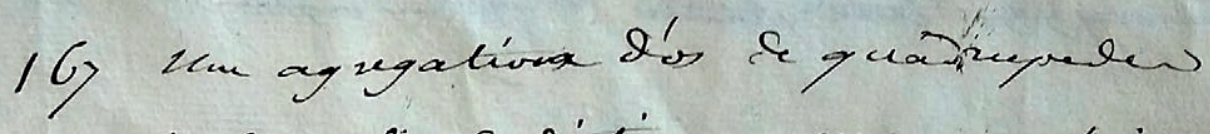

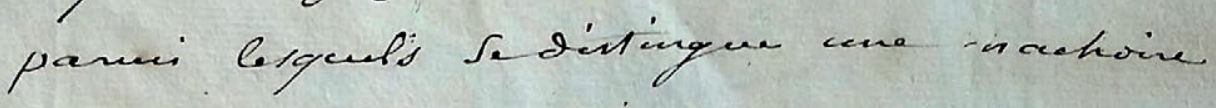
158 the anter tenblable cuais phen pelite

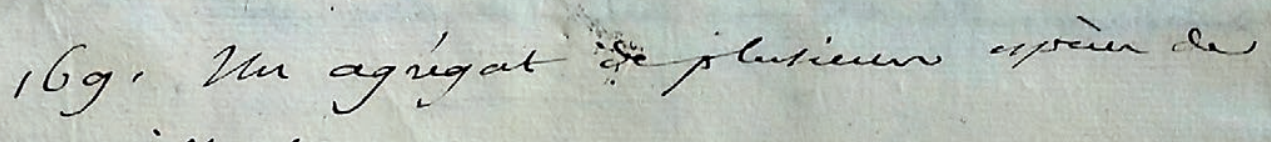
cogevieur.

$170 \mathrm{~cm}$ agnegal de Babanen

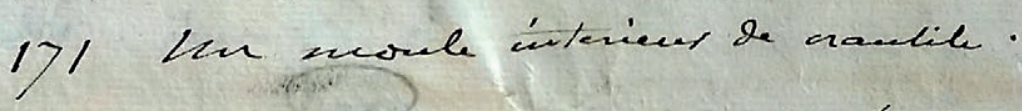
72 un tabjisen a grangs troun $1)$ an tabipon a prebia tron . 174 Quate craber se rapprochant din. calogppar on der Drusuien.

175 Quate excuplain de thombe de val ge Lanca

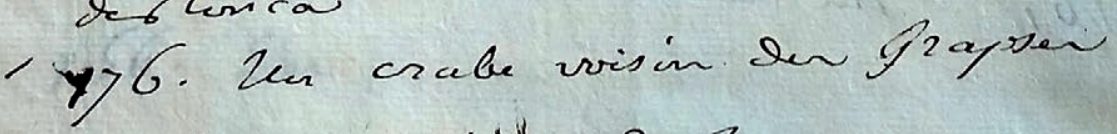
177: Secep cilib de Renca

1) 8 Seap knnitalen

$17 \%$ Luce cansite

14t Seepp,veigum

181 hoir ancmoniter pryitumen. 


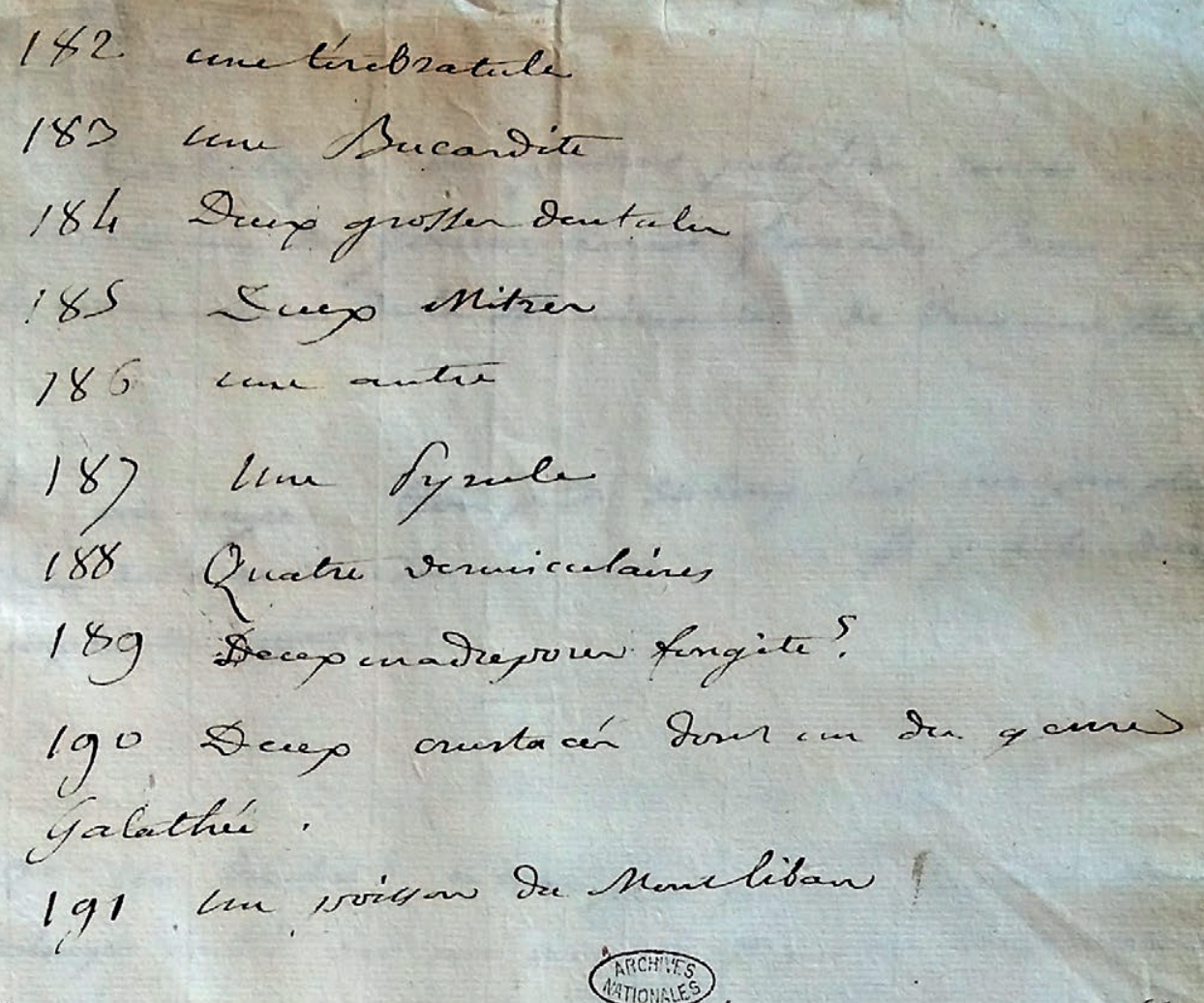

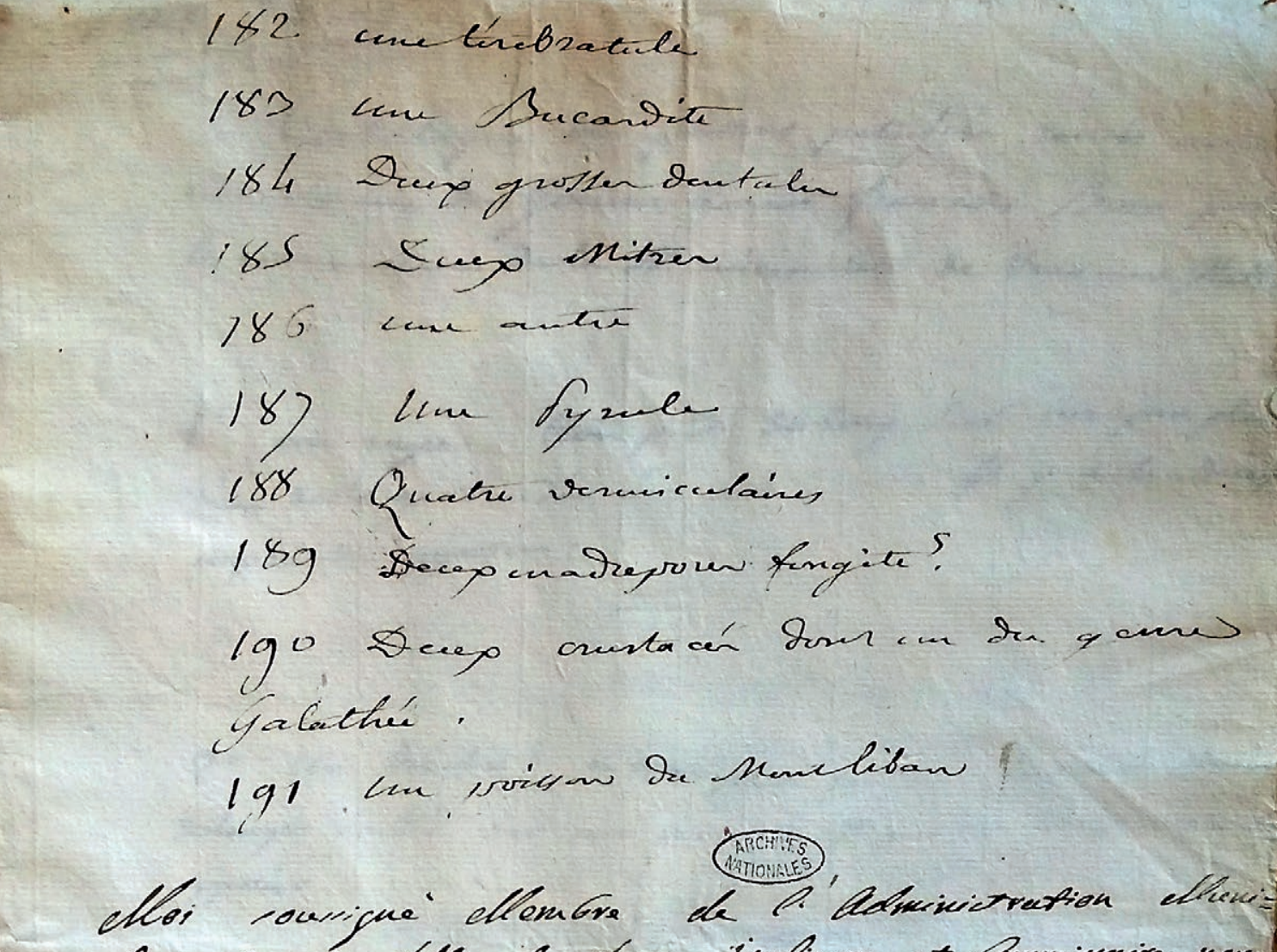

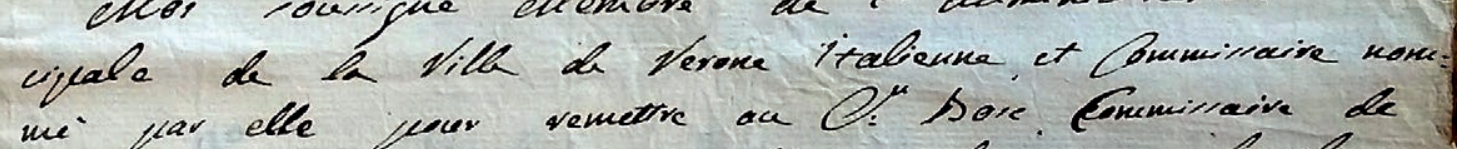

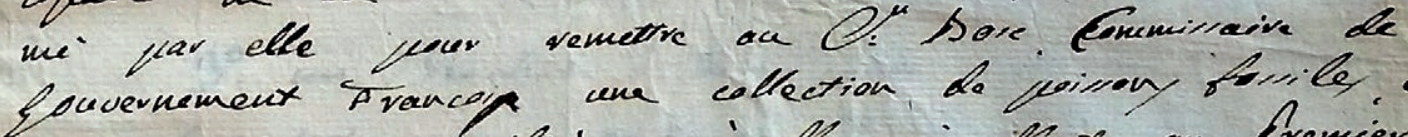

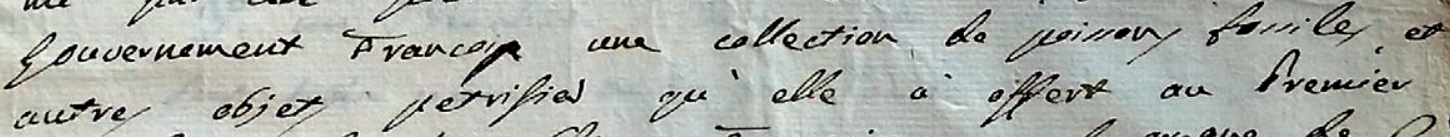

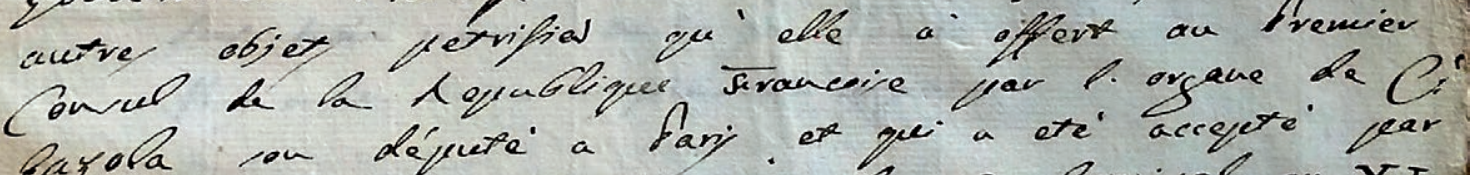

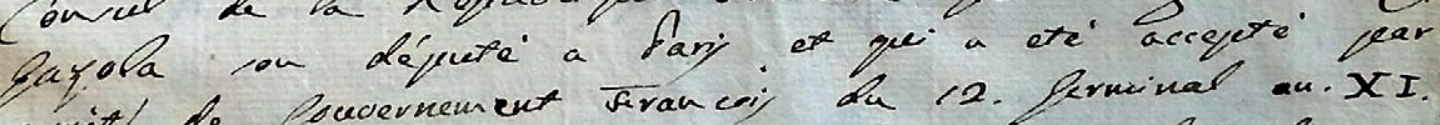

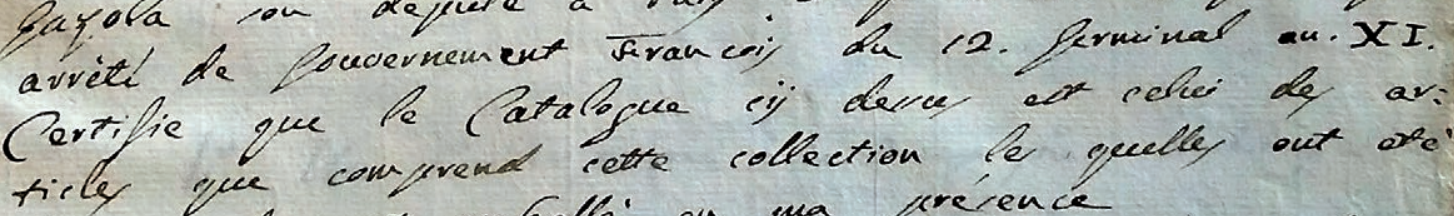
Gien et duement emballe on ma perérence ofealle out ofe

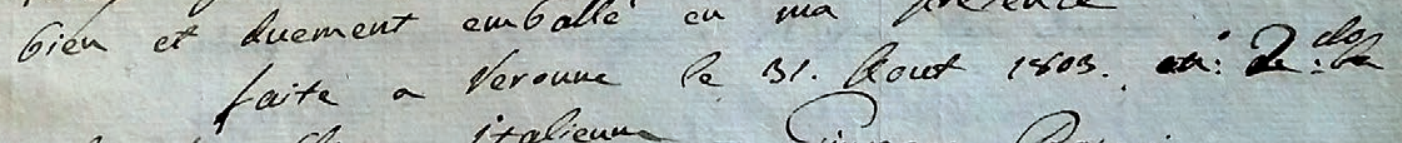

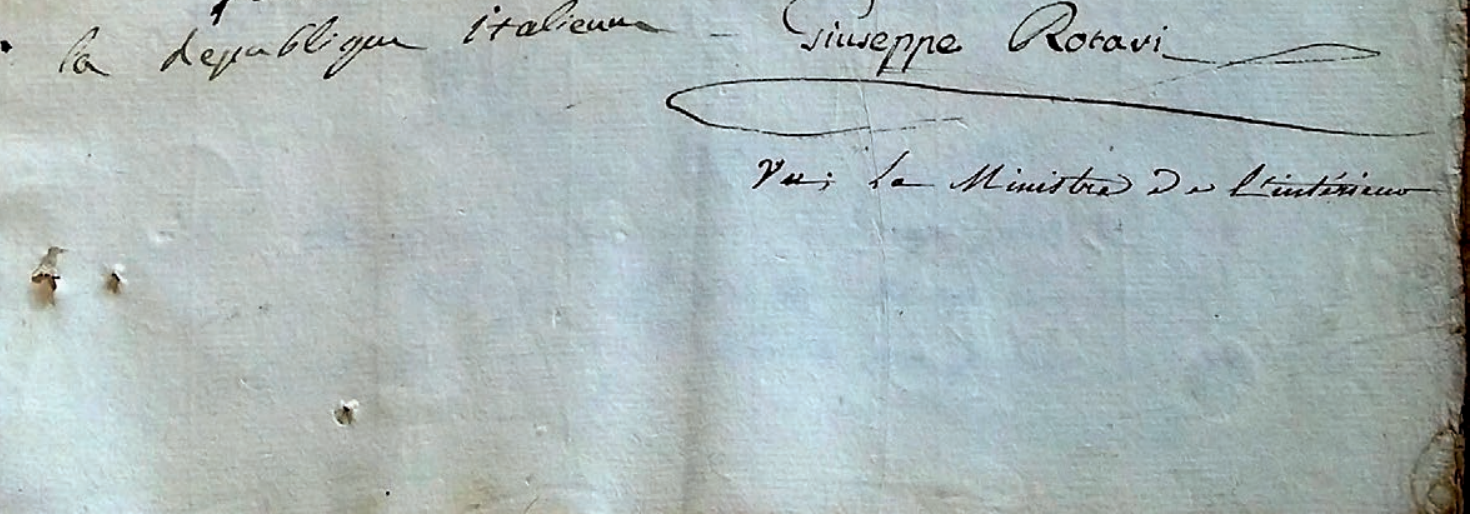

ANNEXE 2. - Suite; catalogue de Louis Augustin Guillaume Bosc, spécimens 182 à 191. 